\author{
Mon ograph \\ urn:1sid:zoobank.org:pub:A32EAE51-9051-458A-81DD-8EA921901CDC
}

\title{
The birds (Aves) of Oromia, Ethiopia - an annotated checklist
}

\author{
Kai GEDEON ${ }^{1, *}$, Chemere ZEWDIE ${ }^{2} \&$ Till TÖPFER $^{3}$ \\ ${ }^{1}$ Saxon Ornithologists' Society, P.O. Box 1129, 09331 Hohenstein-Ernstthal, Germany. \\ ${ }^{2}$ Oromia Forest and Wildlife Enterprise, P.O. Box 1075, Debre Zeit, Ethiopia. \\ ${ }^{3}$ Zoological Research Museum Alexander Koenig, Centre for Taxonomy and Evolutionary Research, \\ Adenauerallee 160, 53113 Bonn, Germany. \\ ${ }^{*}$ Corresponding author: k.gedeon@gmail.com \\ 2Email: nchemere@gmail.com \\ ${ }^{3}$ Email: t.toepfer@leibniz-zfmk.de \\ ${ }^{1}$ urn:lsid:zoobank.org:author:F46B3F50-41E2-4629-9951-778F69A5BBA2 \\ ${ }^{2}$ urn:Isid:zoobank.org:author:F59FEDB3-627A-4D52-A6CB-4F26846C0FC5 \\ ${ }^{3}$ urn:1sid:zoobank.org:author:A87BE9B4-8FC6-4E11-8DB4-BDBB3CFBBEAA
}

\begin{abstract}
Oromia is the largest National Regional State of Ethiopia. Here we present the first comprehensive checklist of its birds. A total of 804 bird species has been recorded, 601 of them confirmed (443) or assumed (158) to be breeding birds. At least 561 are all-year residents (and 31 more potentially so), at least 73 are Afrotropical migrants and visitors (and 44 more potentially so), and 184 are Palaearctic migrants and visitors (and eight more potentially so). Three species are endemic to Oromia, 18 to Ethiopia and 43 to the Horn of Africa. 170 Oromia bird species are biome restricted: 57 to the Afrotropical Highlands biome, 95 to the Somali-Masai biome, and 18 to the Sudan-Guinea Savanna biome. 26 species of birds of Oromia are Threatened (three Critically Endangered, nine Endangered, and 14 Vulnerable), and an additional 20 species are classified as Near Threatened. International regulations affect 225 Ethiopian bird species. Of the polytypic species, a total of 620 subspecies can be taxonomically assigned to Oromia. 11 of them are endemic to Oromia, 71 to Ethiopia and 150 to the Horn of Africa. This very high diversity underlines the high responsibility of the Oromia National Regional State for bird conservation.
\end{abstract}

Keywords. Birds of Ethiopia, Oromia, check-list, taxonomy, conservation.

Gedeon K., Zewdie C. \& Töpfer T. 2017. The birds (Aves) of Oromia, Ethiopia - an annotated checklist European Journal of Taxonomy 306: 1-69. https://doi.org/10.5852/ejt.2017.306

\section{Introduction}

The Federal Republic of Ethiopia is administratively subdivided into nine National Regional States (NRS) and two chartered cities (Fig. 1). Being the largest NRS, accounting for about one quarter of the total area of Ethiopia, Oromia is a region of great physiographical and habitat diversity. Therefore, Oromia is of exceptional importance for Ethiopia's avifauna and precise knowledge about the distribution and conservational needs of its birdlife is crucial for effective protection measures. 
The scientific study of the birds of Oromia is rooted in the explorations of the Horn of Africa that began in the first half of the 19th century. The research of Friedrich Wilhelm Hemprich, Christian Gottfried Ehrenberg and Eduard Rüppell led to the description of many new species, but focused exclusively on areas in modern Eritrea and northern Ethiopia (Amhara, Tigre). It was only in the second half of the 19th century that Theodor von Heuglin became the first ornithologist to penetrate the region of today's Oromia (Heuglin 1868). His work had considerable consequences: while the first systematic index of the birds of Northeast Africa by Rüppell (1845) included some 500 species, Heuglin's subsequent lists (Heuglin 1856, 1869-1874) finally comprised over 900 species. From the late 19th century to the first half of the 20th century numerous scientific expeditions took place, extending the knowledge of the avifauna of Oromia substantially. Among the explorers travelling in Oromia and its borders, significant collections were made by Orazio Antinori, Antonio Cecchi, Frank L. James, Prince Eugenio Ruspoli, Arthur Donaldson Smith, Carlo von Erlanger, Oscar Neumann, Edgar Mearns, Augusto Toschi, Marchese Saverio Patrizi Naro Montoro and Constantine W. Benson. During this time, comprehensive handbooks were published on African birds (Shelley 1896-1912; Reichenow 1901-1905), and on the Horn of Africa (Moltoni \& Ruscone 1940-1944, unfinished).

The first checklist of birds of Ethiopia (including present-day Eritrea) was published in 1971 by Urban \& Brown, while the first distribution atlas by Ash \& Atkins appeared as recently as 2009. With the present

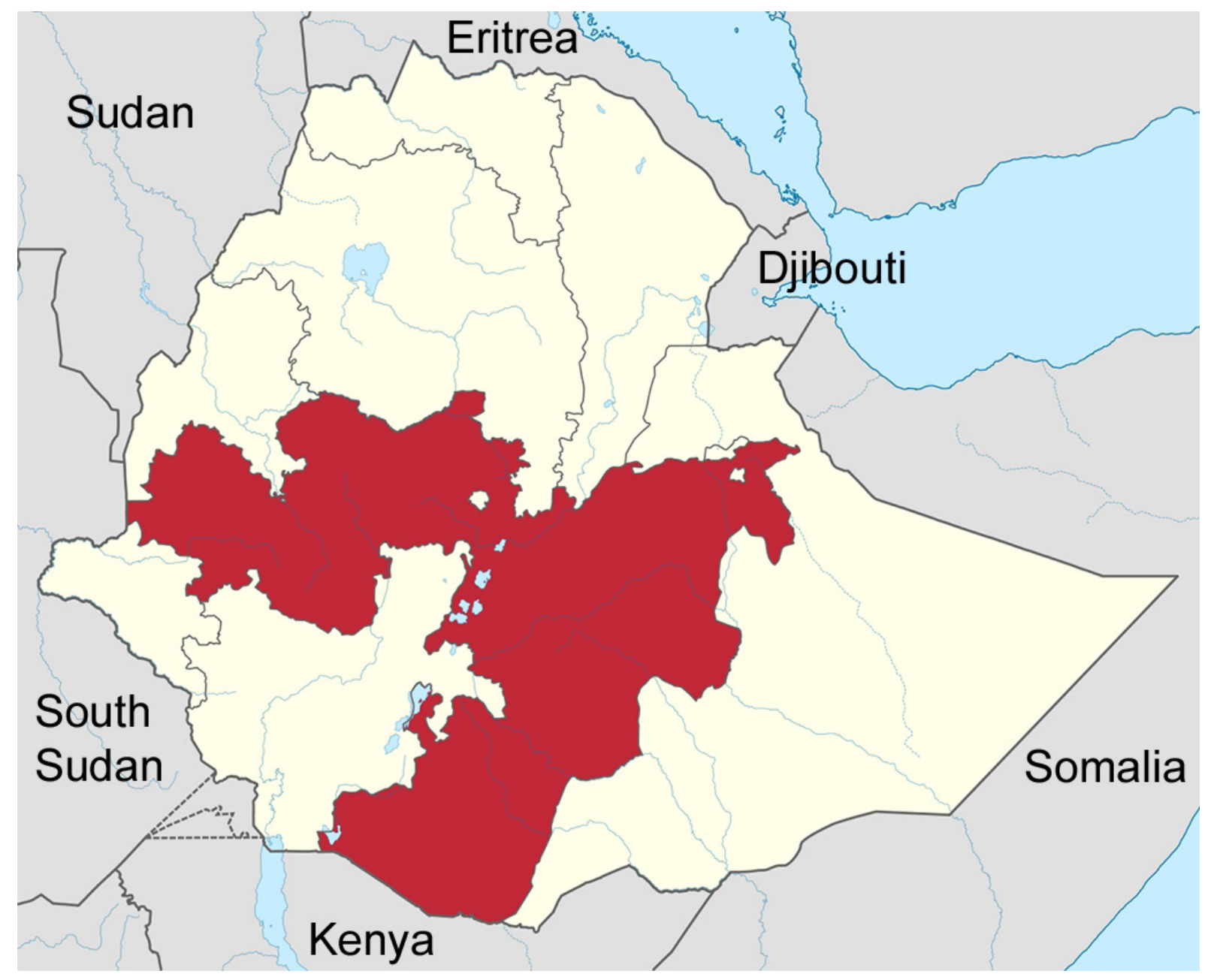

Fig. 1. The National Regional State of Oromia (red) within Ethiopia and the Horn of Africa (boundaries after MapLibrary 2013). 
checklist, being the first for a single Ethiopian NRS, we not only address incomplete knowledge about the distribution or the taxonomic position of particular bird taxa in Oromia but also explicitly consider the subspecific level for the first time. We agree with Collar \& Stuart (1985) that the environmental crisis of our planet is so great now that focusing merely on species means failing to recognize the considerable loss of genetic diversity we are about to sustain at the subspecific level. Thus, with this checklist we also seek to contribute to a broader knowledge of Ethiopian birds and to support ongoing conservation activities. As the current constitution of Ethiopia guarantees substantial authority to each NRS, with regional parliaments having legislative and executive powers to regulate their own internal affairs, both the central government and the regional governments are responsible for the protection and management of natural resources and protected areas (Vreugdenhil et al. 2012). We sincerely hope that our checklist will be used as a basis to conduct further research.

\section{Material and methods}

The checklist consists of two parts: the actual species list (Appendix 1) and the annotations (Appendix 2). Appendix 1 covers basic distributional information and data on conservation etc., while Appendix 2 comprises detailed commentaries on taxonomic issues and/or further research needs. Additionally, for the first time in a study of such scope we have assigned individual subspecies to biomes following Fishpool \& Evans (2001).

\section{Study site}

The total area of the Oromia NRS is $284538 \mathrm{~km}^{2}$ (Central Statistical Agency of Ethiopia 2012; Fig. 1) which corresponds to about $26 \%$ of Ethiopia's surface area. Regarding major biotic communities, of the 15 African biomes, characterized by the dominant forms of plant life and the prevailing climate (Fishpool \& Evans 2001), three are to be found in Oromia: the Afrotropical Highlands biome, the SomaliMasai biome, and the Sudan-Guinea Savanna biome (Fig. 2). About $44 \%$ of all bird species regularly occurring in Africa are confined to a single biome (Birdlife International 2008). A recent statistical analysis (Linder et al. 2012) shows that the Somali-Masai biome and the Sudan-Guinea Savanna biome are clearly delineated on grounds of their birdlife, whereas the Afrotropical Highlands do not constitute a continuous biogeographic unit across Africa. However, the study recognizes the Ethiopian Highland region as a biogeographically distinct unit (Linder et al. 2012).

The Highlands of Oromia include the mountain ranges east and west of the Rift Valley. The highest peak is Mt. Tullu Deemtu (4377 m a.s.1.) in the Bale Mountains. Outside of protected zones the area is densely populated and heavily used for agriculture, particularly coffee and various cereals. Today only a few remnants of the highlands' original forest cover remain. The middle Awash region in north-eastern Oromia, the Rift Valley and the territories south and southeast of the Bale Mountains are assigned to the Somali-Masai biome. This is primarily a savanna landscape, extending from around $600 \mathrm{~m}$ a.s.l. at the Awash and Chew Bahir to $1800 \mathrm{~m}$ a.s.l. on the Borana plateau. The area is, apart from the Rift Valley, sparsely populated and primarily used pastorally. The Sudan-Guinea Savanna biome extends from the west to the highlands of Oromia. The region includes savanna and deciduous woodlands. It is less densely populated than the higher areas and mainly used for agriculture.

Major rivers are the Awash, Wabe-Shebele, Genale, Gibe, Baro, Dedessa and Guder. Besides some smaller crater lakes, the large Rift Valley lakes (e.g., Koka, Zway, Abiyiatta, Shalla and Langano) are particularly important as breeding, roosting and wintering sites for birds.

In Oromia there are six National Parks, some of which are not fully established yet (Vreugdenhil et al. 2012): Awash National Park $\left(756 \mathrm{~km}^{2}\right.$, shared with Afar NRS, established 1958, federally managed), Dhati-Welel National Park $\left(1031 \mathrm{~km}^{2}\right.$, originally controlled hunting, established as a park in 2010, regionally managed), Bale Mountains National Park $\left(2200 \mathrm{~km}^{2}\right.$, established 1962, federally 
managed), Borena National Park $\left(1500 \mathrm{~km}^{2}\right.$, the previous Yabello Wildlife Sanctuary, which was initially almost $2500 \mathrm{~km}^{2}$, upgraded to a National Park in 2013 and renamed Borena National Park, regionally managed), Abijiatta Shalla National Park $\left(887 \mathrm{~km}^{2}\right.$, established 1963, federally managed), Arsi Mountains National Park (pending approval, four units, incl. former Deara Sanctuary, regionally managed). Two other protected areas have the status of Wildlife Sanctuaries and are designed to protect large mammals (Babille Elephant Sanctuary, shared with the Somali Region, and Sinkele Swayne's Hartebeest Sanctuary, shared with the Southern Nations, Nationalities, and Peoples' Region). Both are federally managed. Furthermore, there is a number of formally protected National Forest Priority Areas. A comprehensive depiction and analysis of the current system of protected areas in Ethiopia, as well as proposals for its development, has been submitted by Vreugdenhil et al. (2012).

The Birdlife International "Important Bird Areas" (Fishpool \& Evans 2001) as well as the "Endemic Bird Areas" (Stattersfield et al. 1998) are not subject to legal protection.

\section{Data collection}

For this checklist we largely relied on Ash \& Atkins (2009) who present the first comprehensive appraisal of ornithological data from Ethiopia and Eritrea. Our evaluation included all 1-degree tetrads where Oromia has a share of at least $10 \%$, and in which the respective species was observed (Fig. 3). We

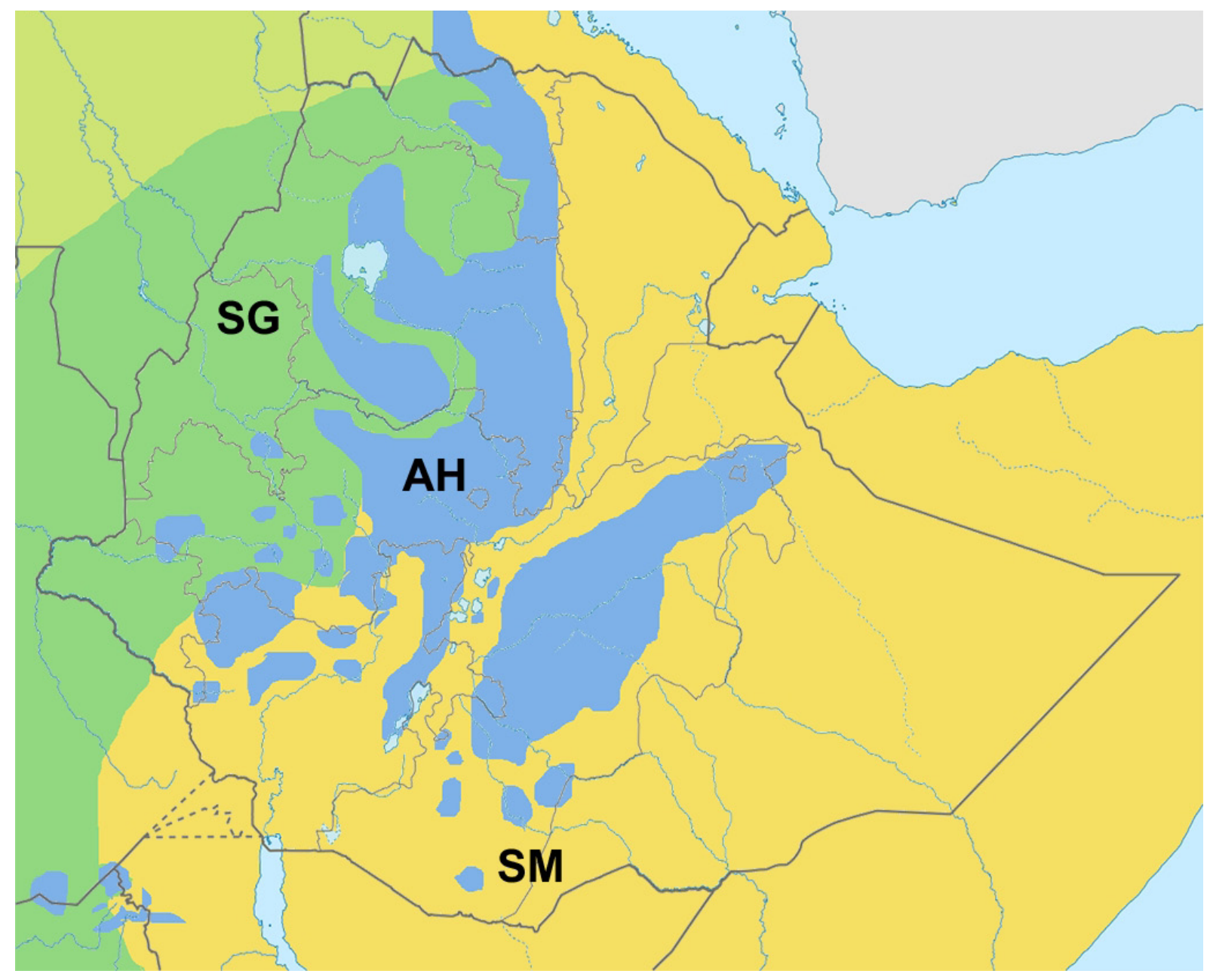

Fig. 2. Biomes in the Horn of Africa following Fishpool \& Evans (2001). SG = Sudan-Guinea Savanna biome (green); $\mathrm{AH}=$ Afrotropical Highlands biome (blue); $\mathrm{SM}=$ Somali-Masai biome (yellow). 
also consulted the seven-volume Birds of Africa (Brown et al. 1982; Fry et al. 1988; 2000, 2004; Keith et al. 1992; Urban et al. 1986, 1997) and the sixteen-volume "Handbook of the Birds of the World" (del Hoyo et al. 1992-2011). Further literature was considered if necessary for the clarification of details or if published after Ash \& Atkins (2009). Otherwise, we referred to the extensive bibliography contained therein. Regarding the distribution of birds throughout neighbouring countries we consulted Ash \& Miskell $(1983,1998)$ for Somalia, Lewis \& Pomeroy (1989) for Kenya, and van den Elzen \& König (1983) and Nikolaus (1987) for Sudan.

\section{Nomenclature}

The nomenclature and order of taxa including their respective numbers follow Clements et al. (2015) and the species numbers used by Ash \& Atkins (2009) are given as well. All hitherto identified bird species of Oromia are listed in the checklist and annotations are made concerning pending taxonomic changes not implemented in Clements et al. (2015). Occasionally, different assessment of taxa by del Hoyo \& Collar (2014) was not considered in order to maintain consistent taxonomic treatment, as the second part of their list had not been published. Subspecies are listed in Appendix 2, however, notes on monotypic species are only made if further explanation was necessary. Hybrids and records in question were not considered, for such cases the lists B and C in Ash \& Atkins (2009) should be consulted.

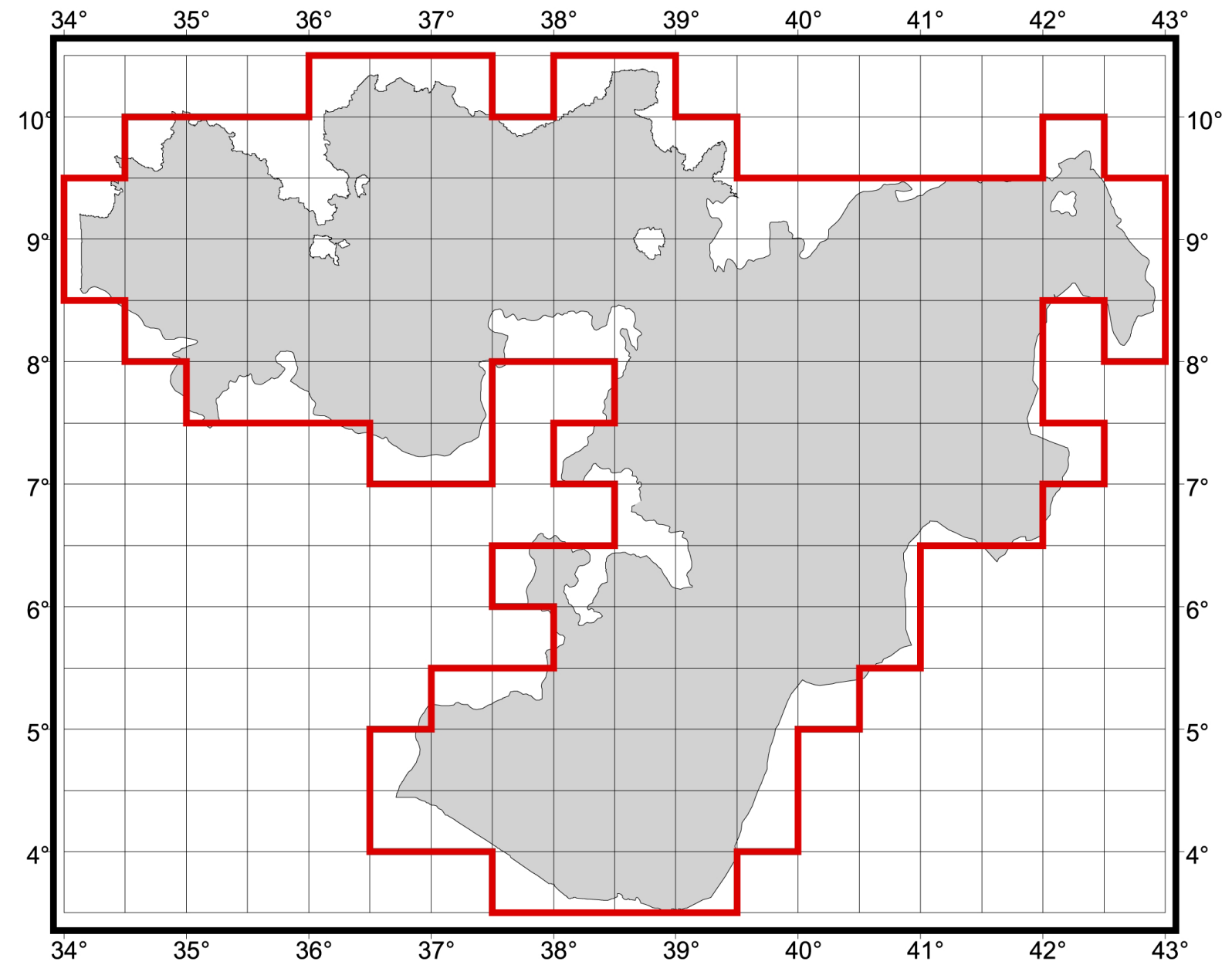

Fig. 3. The National Regional State of Oromia covered by 1-degree-tetrads. For the bold-red framed tetrads the data on distribution of birds was taken from the distribution atlas of Ash \& Atkins (2009). 


\section{Status of occurrence}

As to the status, we distinguish between all-year residents (species which are present throughout the year, although fluctuations during the year and local/regional movements are possible), Afrotropical migrants and visitors (species which are seasonally absent due to movements within tropical sub-Saharan Africa, incl. vagrants), and Palaearctic migrants and visitors (species which are seasonally absent due to movements between tropical sub-Sahara Africa and the Palaearctic region; direct evidence through banding, telemetry; species or subspecies affiliation and/or indirect indicators such as fluctuations in numbers of individuals during the year or in irregular periods, incl. vagrants). Furthermore, we assessed the breeding of each taxon. Evidence of breeding included occupied nests, recently fledged juveniles, eggs in ovaries and the like. Breeding is assumed if no verified breeding records in Oromia exist so far, but reproduction there is most likely. All classifications are based on data in Ash \& Atkins (2009).

\section{Abundance}

In assessing abundance of taxa we have combined the ten classifications of Ash \& Atkins (2009) into three broader groups: "vagrant or rare" ("vagrant" = 1-2 records, "very rare" = few records, "rare" = unlikely to be recorded), "uncommon" ("very uncommon" = recorded on 5-20\% of occasions, "uncommon" = recorded on $21-30 \%$ of occasions, "reasonably common" = recorded on $31-45 \%$ of occasions, "fairly common" = recorded on 46-60\% of occasions), and "common" ("common" = usually recorded, "very common" = an intermediate category, "abundant" = common in large numbers). Where necessary, we have adjusted the classification for the whole of Ethiopia and Eritrea by Ash \& Atkins (2009) in order to reflect the situation in Oromia.

\section{Endemism and biome restriction}

For each species and subspecies we have identified whether its occurrence is restricted to either Oromia, Ethiopia, or the Horn of Africa, or to one of the three biomes. We have assigned the category "restricted to Oromia" even if small parts of the total range lie outside the Oromia NRS. Attribution of species to Oromia and Ethiopia is based on the distribution maps in Ash \& Atkins (2009). "Horn of Africa" refers to Ethiopia, Eritrea, Somalia, Djibouti and Socotra. The assignment of taxa to the latter region is based mainly on distribution maps in The Birds of Africa, Vols 1-7 (Brown et al. 1982; Fry et al. 1988, 2000, 2004; Keith et al. 1992; Urban et al. 1986, 1997). Biome boundaries correspond to those given by Fishpool \& Evans (2001). When assigning species to biomes we largely followed Ash \& Atkins (2009) and have come to other assessments only occasionally.

\section{Legal protection/regulations}

The present checklist should also serve as a guide to the implementation of practical conservation measures. We have therefore included information on the legal protection status of each species according to the relevant sources. Ethiopia is a party to the following international agreements: the Convention on the Conservation of Migratory Species of Wild Animals (CMS, effective 23 Feb. 2012), the Agreement on the Conservation of African-Eurasian Migratory Waterbirds (AEWA, adopted Sep. 2008), and the Convention on International Trade in Endangered Species of Wild Fauna and Flora (CITES, valid from 3 Apr. 2012). At national level, the government of Ethiopia regulates the designation of protected areas, the utilization of wildlife, and the respective responsibilities applying the Development, Conservation and Utilization of Wildlife Proclamation No. 541/2007. Specific regulations for protection, use and hunting of wild species in Oromia are found in the Council of Ministers Regulations to Provide for Wildlife Development, Conservation and Utilization No. 163/2008 in which protected, tradable and huntable species are defined. 


\section{Results}

\section{Species}

The species list (Appendix 1) includes a total of 804 species recorded in Oromia. Ash \& Atkins (2009) list a total of 872 species for Ethiopia and Eritrea. Accordingly, around $92 \%$ of these species are represented in Oromia, although Oromia comprises only about a quarter of the territory of the two countries.

Of the 804 species, 601 of them are confirmed (443) or assumed (158) to be breeding birds; at least 561 are all-year residents (and 31 more potentially so), at least 73 are Afrotropical migrants and visitors (and 44 more potentially so), and 184 are Palaearctic migrants and visitors (and eight more potentially so).

Of the listed species, three are endemic to Oromia (Table 1), 18 are endemic to Ethiopia and 43 are endemic to the Horn of Africa (Appendix 1). Of the latter, ten have offshoots of their ranges into Kenya and/or Sudan and South Sudan. According to Redman et al. (2011) there is a total of 61 endemic bird species in the Horn of Africa, about 70\% of which are found in Oromia. At least 170 bird species known from Oromia are biome-restricted: 57 to the Afrotropical Highlands biome, 95 to the Somali-Masai biome, and 18 to the Sudan-Guinea Savanna biome.

The International Union for Conservation of Nature (IUCN) Red List names 26 species from Oromia (three Critically Endangered, nine Endangered, 14 Vulnerable). In total, 225 species are affected by international regulations and fall within at least one of the following agreements: CMS (nine species), AEWA (134 species) and CITES (97 species). Under national law, 17 species are protected, 122 species are tradable, and 47 species are huntable.

\section{Subspecies}

About 41\% (327) of Oromia's 804 bird species are monotypic. Since some of the remaining 477 polytypic species are represented in the area by more than one subspecies, the total number of nominal

Table 1. Endemic or nearly endemic bird taxa of Oromia. The scientific names follow Clements et al. (2015). Taxon numbering corresponds to Clements et al. (2015) and Ash \& Atkins (2009), respectively (separated by slash).

\begin{aligned} & \hline \multicolumn{1}{c}{ Taxon no. } Species name \\ & \hline $1230 / 12$ Pternistis castaneicollis atrifrons (Conover, 1930) \\ & $1332 / 6$ Scleroptila psilolaema psilolaema (G.R. Gray, 1867) \\ & $5776 / 329$ Tauraco leucotis donaldsoni (Sharpe, 1895) \\ & $5777 / 337$ Tauraco ruspolii (Salvadori, 1896) \\ & $20014 / 503$ Zavattariornis stresemanni Moltoni, 1938 \\ & $20762 / 553$ Calandrella somalica megaensis Benson, 1946 \\ & $20862 / 557$ Galerida theklae huei Érard \& de Naurois, 1973 \\ & $21134 / 528$ Hirundo megaensis Benson, 1942 \\ & $23234 / 613$ Phylloscopus umbrovirens omoensis (Neumann, 1905) \\ & $24127 / 567$ Cisticola robustus omo Neumann \& Lynes, 1928 \\ & $24532 / 634$ Sylvia lugens griseiventris Érard, 1978 \\ & $25604 / 638$ Turdoides aylmeri boranensis (Benson, 1947) \\ & $26451 / 684$ Cossypha semirufa donaldsoni Sharpe, 1895 \\ & $29094 / 847$ Anthus caffer australoabyssinicus Benson, 1942 \\ & \hline\end{aligned}


Table 2. Summary of the bird species and subspecies found in Oromia. The species without subspecific assignment are Columba livia Gmelin, 1789, Sylvia borin (Boddaert, 1783), S. nisoria (Bechstein, 1795) and Chalcomitra hunteri (Shelley, 1889).

\begin{tabular}{lc}
\hline Species & Nominal taxa \\
\hline 327 monotypic species & 327 \\
364 with 1 subspecies & 364 \\
81 with 2 subspecies & 162 \\
22 with 3 subspecies & 66 \\
5 with 4 subspecies & 20 \\
1 with 6 subspecies & 6 \\
4 without classification & 4 \\
\hline
\end{tabular}

taxa is at least 945 (Table 2). Overall, 620 of the subspecies can be taxonomically assigned to Oromia with sufficient accuracy. Of these subspecies, 11 are restricted to Oromia (Table 1), 71 to Ethiopia, and 150 to the Horn of Africa, although 36 of them have offshoots of their ranges into Kenya and/or Sudan and South Sudan.

\section{Discussion}

Although the number of Ethiopian bird taxa recorded in Oromia is comparatively high, the status and the ranges of many species and subspecies are not adequately known yet. Future studies should address these gaps, which is why we put forward various suggestions for further research as given in Appendix 2.

With three bird species and 11 subspecies endemic to Oromia (Table 1), plus an additional 18 species and 71 subspecies endemic to Ethiopia, and a total of 804 species known up to now, the Oromia NRS has a very high level of responsibility in the protection of bird diversity. A number of range-restricted species, such as Bostrychia carunculata (Rüppell, 1837), Columba albitorques (Rüppell, 1837), Agapornis taranta (Stanley, 1814), Melaenornis chocolatinus (Rüppell, 1840) or Serinus tristriatus (Rüppell, 1840), are still numerous and/or occur in human-modified habitats so that no special protection measures are currently required. For six groups of birds, however, there is specific and often urgent need for action to ensure their conservation. These groupings are based on hitherto identified common threats, e.g., to certain habitats, population size or lifestyle.

(1) Birds of the highland grasslands and moorlands, e.g., Cyanochen cyanoptera (Rüppell, 1845), Tadorna ferruginea (Pallas, 1764), Scleroptila psilolaema psilolaema (G.R. Gray, 1867), Vanellus melanocephalus (Rüppell, 1845), Pyrrhocorax pyrrhocorax baileyi Rand \& Vaurie, 1955 and Macronyx flavicollis Rüppell, 1840. These birds naturally have a small distribution in Oromia. Their habitats are often still intact, but are expected to change in the course of global warming (McSweeney et al. 2010). It should be a main objective to secure sufficiently large protected areas that are not substantially affected by humans. The Bale Mountains National Park and the proposed Arsi Mountains National Park play important roles in a coherent system of protected areas.

(2) Birds of forests, woodlands and river beds, e.g., Pternistis erckelii (Rüppell, 1835), Pternistis harwoodi (Blundell \& Lovat, 1899), Pternistis castaneicollis atrifrons (Conover, 1930) (Fig. 4), 
Tauraco leucotis donaldsoni (Sharpe, 1895), Tauraco ruspolii (Salvadori, 1896) and Salpornis salvadori erlangeri Neumann, 1907. The habitats of these birds are under extreme pressure due to various forms of exploitation. The natural forest cover in the South Central Rift Valley area declined from 16\% in 1972 to $2.8 \%$ in 2000 , caused by small-scale agriculture, commercial logging, and commercial farms (Dessie \& Kleman 2007). The existing system of National Priority Forest Areas is obviously not a sufficient instrument to prevent the disappearance or deterioration of habitats of forest and woodland birds. New approaches to prevent deforestation like community-based forest management are needed but only effective if adequately monitored and supervised (Takahashi \& Todo 2012).

(3) Birds of open savannas, e.g., Zavattariornis stresemanni Moltoni, 1938 (Fig. 5), Heteromirafra archeri Clarke, 1920, Calandrella somalica megaensis Benson, 1946, Hirundo megaensis Benson, 1942 (Fig. 6). The habitats of these birds are also under considerable exploitation pressure. Essential causes are increasing population density, overgrazing, encroachment, ban of rangeland burning and the conversion of pastures into farmland (Homann 2005; Gemedo-Dalle et al. 2006). These birds show a clear reliance on modified habitats, some obviously within narrow climatic limits (Donald et al. 2012). Traditional pastoral land use promotes their existence, and this must be considered in the management within and outside protected areas. Bassi (2010) and Bassi \& Tache (2011) argue that strengthening customary governance holds the best prospect for improving the situation of both the pastoralists and the environment.

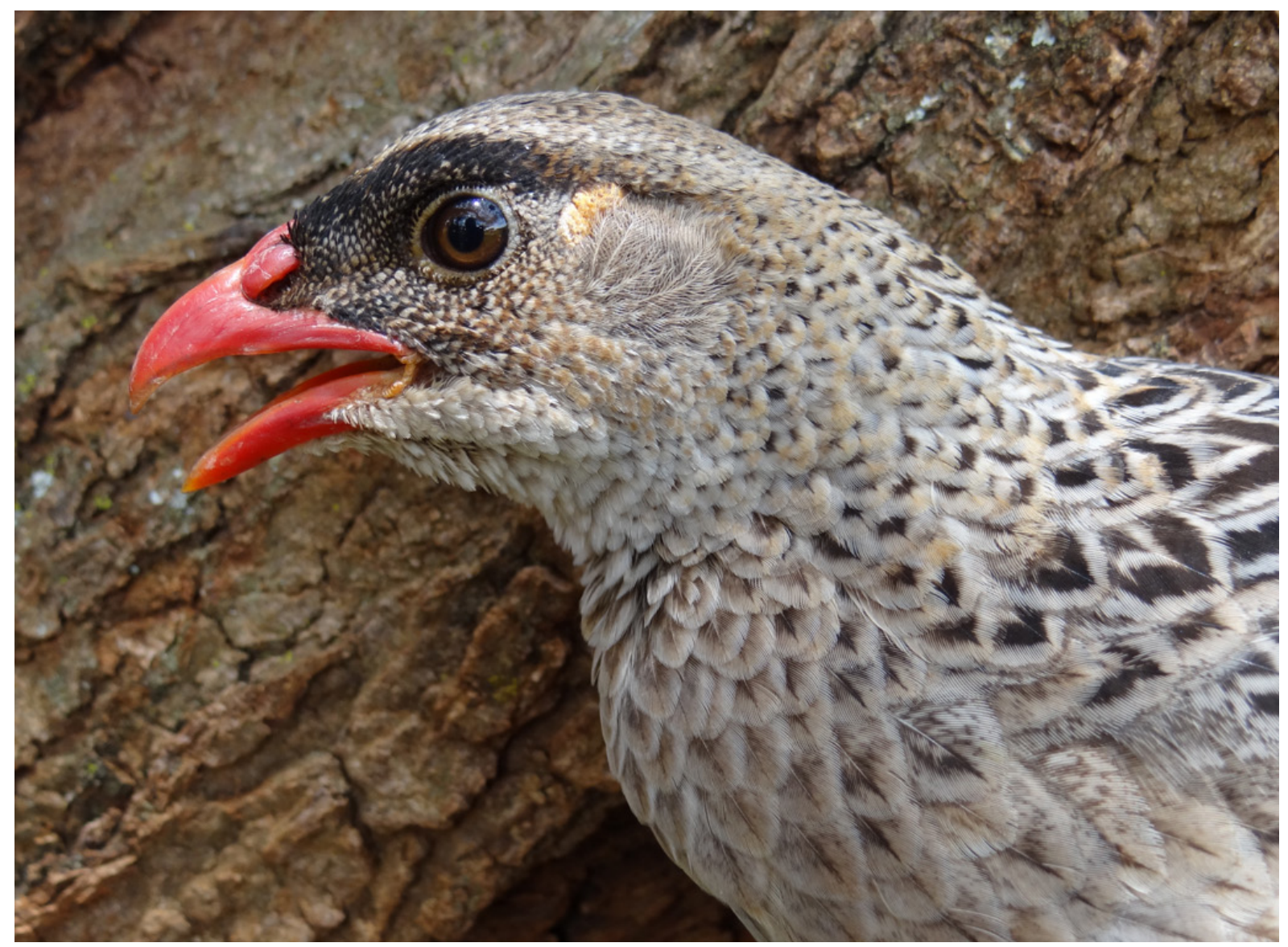

Fig. 4. The Black-fronted Francolin Pternistis castaneicollis atrifrons (Conover, 1930) exists in a small mountain range around Mega in southern Oromia (Borana zone). It shows a number of distinct features that may justify the split from P. castaneicollis (Photo: Kai Gedeon). 
(4) Migratory waterbirds, e.g., Anas clypeata Linnaeus, 1758, Anas acuta Linnaeus, 1758, Anas querquedula Linnaeus, 1758, Anas crecca Linnaeus, 1758, Aythya nyroca (Güldenstädt, 1770), Aythya fuligula (Linnaeus, 1758), Phoenicopterus roseus Pallas, 1811, Phoeniconaias minor (E. Geoffroy SaintHilaire, 1798), Pelecanus onocrotalus Linnaeus, 1758, Pelecanus rufescens Gmelin, 1789, Plegadis falcinellus (Linnaeus, 1766), Platalea leucorodia Linnaeus, 1758 and Grus grus (Linnaeus, 1758). Of the great lakes of the Rift Valley, only Lake Abijiatta and Lake Shalla are protected as a conjoint National Park. The park has one of the highest wetland bird diversities in Ethiopia but is in an extremely critical state in terms of its conservation status (Ethiopian Wildlife and Natural History Society 2008/2009). Immediate action is urgently needed. Official protected-area status should be given to other lakes and wetlands as well, and this should be supported by an effective management. According to a recent study by the Ethiopian Wildlife and Natural History Society (2011), the most important areas are Chelekleka Wetland in Debre Zeit, a roosting site for more than 18000 Grus grus cranes from October to March, Green Lake (10 km southeast of Debre Zeit), Lake Zway and Lake Langano. Smaller and more isolated waters (e.g., Horo Bake near Yabello) are also relevant because they serve as important stepping-stones for migratory species. They should be protected by the local authorities.

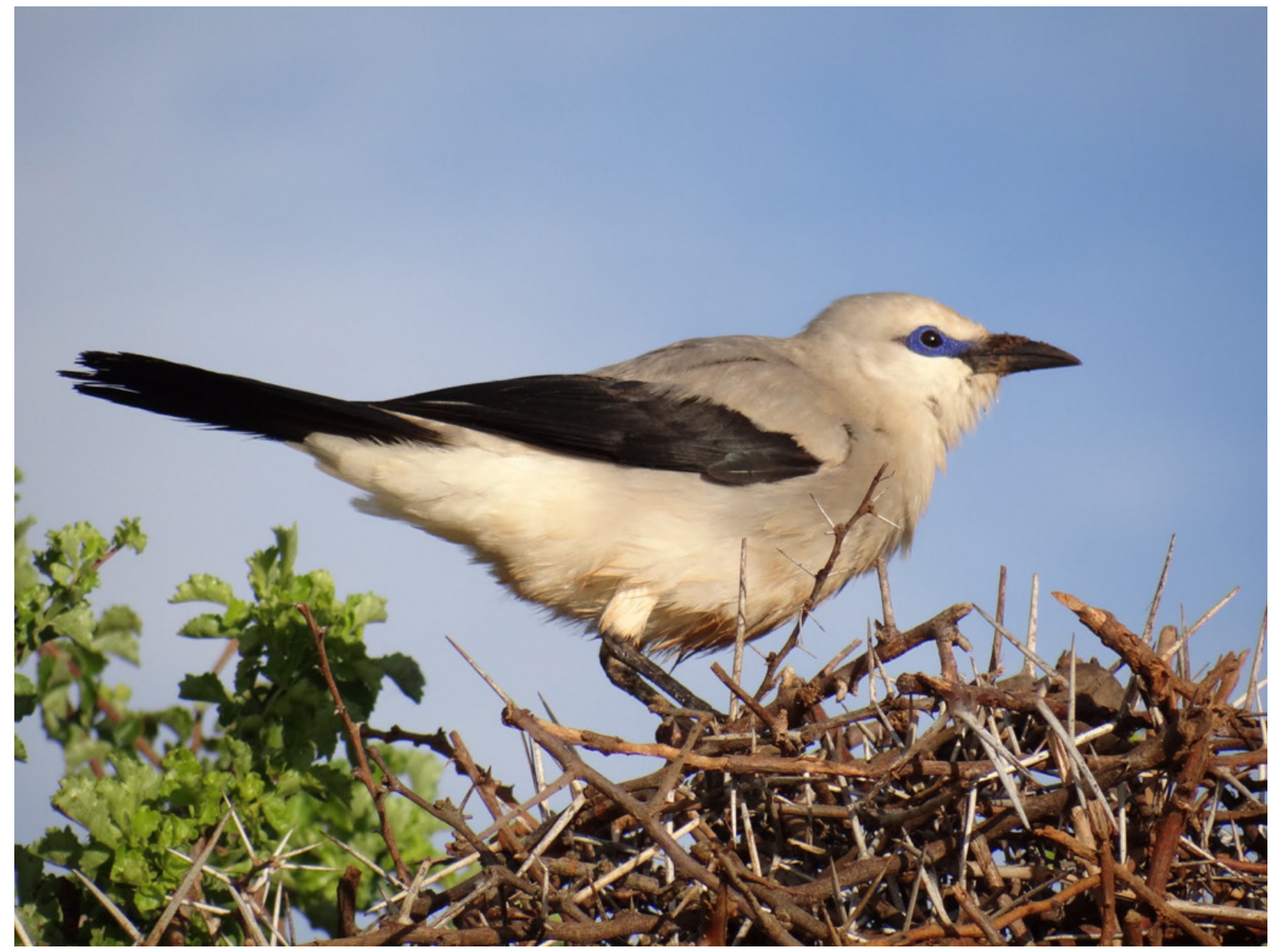

Fig. 5. The Ethiopian Bush-crow Zavattariornis stresemanni Moltoni, 1938 is restricted to the Borana zone in SE Oromia, with a total range of just $6000 \mathrm{~km}^{2}$ (Photo: Kai Gedeon). 
(5) Patchily distributed, often rare, and globally endangered species, including Palaearctic visitors, e.g., Balaeniceps rex Gould, 1850, Geronticus eremita (Linnaeus, 1758), Sarothrura ayresi (Gurney, 1877), Sagittarius serpentarius (J.F. Miller, 1779), Balearica pavonina (Linnaeus, 1758), Bugeranus carunculatus (Gmelin, 1789), Vanellus gregarius (Pallas, 1771), Acrocephalus griseldis (Hartlaub, 1891), Cercomela dubia (Blundell \& Lovat, 1899), Carduelis ankoberensis (Ash, 1979), Serinus flavigula Salvadori, 1888 and Serinus xantholaemus Salvadori, 1896. The scattered ranges of these species are poorly covered by the existing system of protected areas in Oromia, which essentially consists of the establishment and management of large National Parks or sanctuaries. Nonetheless, smaller-scaled areas may equally require effective protection. In these cases, new legal and administrative strategies need to be found to improve the conservation status of these species.

(6) Still widespread and at least locally abundant species for which a significant decrease in population and/or distribution is to be feared. This especially includes the various species of vultures, e.g., Gypaetus barbatus (Linnaeus, 1758), Neophron percnopterus (Linnaeus, 1758), Trigonoceps occipitalis (Burchell, 1824), Torgos tracheliotos (J.R. Forster, 1796), Necrosyrtes monachus (Temminck, 1823), Gyps africanus Salvadori, 1865 and Gyps rueppelli (A.E. Brehm, 1852). Massive declines in abundance were recently documented for seven of eight scavenging raptor species in neighbouring Kenya, apparently as a result of land-use changes and pesticide use, e.g., Carbofuran (Otieno et al. 2011; Virani et al. 2011).

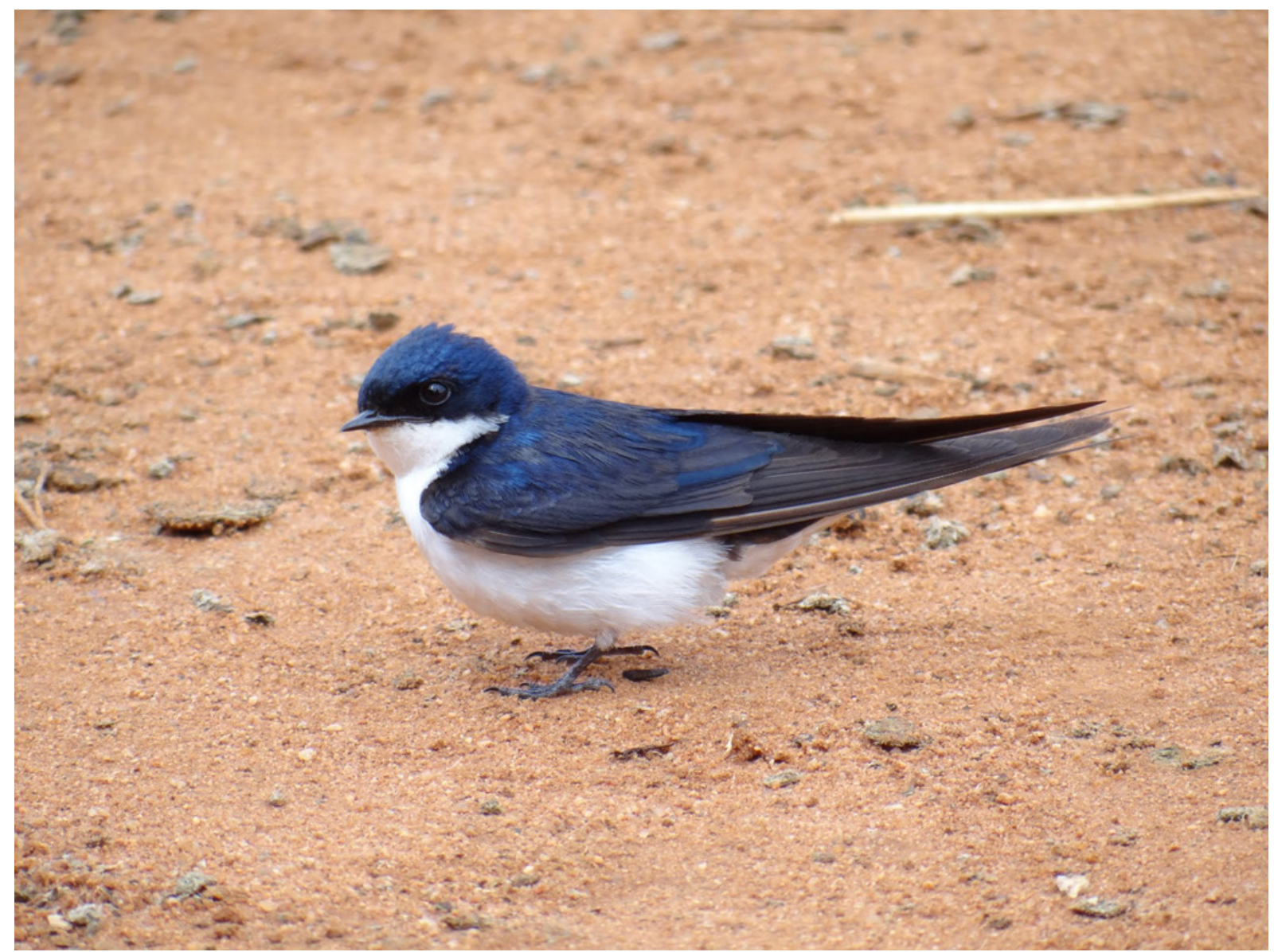

Fig. 6. The White-tailed Swallow Hirundo megaensis Benson, 1942 is another endemic species to Oromia, restricted to the Borana and Guji zone (Photo: Kai Gedeon). 
Because Carbofuran is also used in Ethiopia, it should be banned immediately (Arkumarev et al. 2014). Another serious risk is unsecured power lines. More studies on the extent of this threat, similar to those by Arkumarev et al. (2014) and by Smallie \& Virani (2010) for Kenya, are urgently needed

Thus, an important priority for practical conservation actions would be the sustainable and proper management of conservation areas. The stakeholders involved in nature conservation in Ethiopia will face major challenges in the coming years, not least against the background of new land acquisition schemes (Abbink 2011). In its implementation both economic and social interests as well as the protection of biodiversity must be reconciled.

\section{Acknowledgements}

Ali Guche, Okotu Dida, Tesfaye Mekonnen and Awel Mohammed assisted us during extensive travels in various parts of Ethiopia. We wish to thank them as well as Stefan Ellermann and Heiner Nagel for support in the preparation of maps and figures, and Nigel Redman for his comments and suggestions on the draft version of this checklist. We thank Stefanie Rick for the modification of the tables and for her comments on the text. We also wish to express our sincere gratitude to the referees of the manuscript, John D. Atkins, Michel Louette and Michael S.L. Mills, for their extensive and meticulous reviews. C.Z. is grateful for the support provided by OFWE (Oromia Forest and Wildlife Enterprise).

\section{References}

Abbink J. 2011. 'Land to the foreigners': economic, legal, and socio-cultural aspects of new land acquisition schemes in Ethiopia. Journal of Contemporary African Studies 4: 513-539. https://doi.org $\underline{10.1080 / 02589001.2011 .603213}$

Andrews R. 2015. African Black Swifts Apus barbatus. Bulletin of the African Bird Club 22: 242.

Arkumarev V., Dobrev V., Yilma D.A., Popgeorgiev G \& Nikolov S.C. 2014. Congregations of wintering Egyptian Vultures Neophron percnopterus in Afar, Ethiopia: present status and implications for conservation. Ostrich 85: 139-145. https://doi.org/10.2989/00306525.2014.971450

Ash J.S. \& Atkins J. 2009. Birds of Ethiopia and Eritrea - An atlas of distribution. Christopher Helm, London.

Ash J.S. \& Miskell J.E. 1983. Birds of Somalia, Their habitat, status and distribution. EANHS Ornithological Sub-Committee, Nairobi.

Ash J.S.\&, Miskell J.E. 1998. Birds of Somalia. Pica Press, Sussex.

Bassi M. 2010. The politics of space in Borana Oromo, Ethiopia: demographics, elections, identity and customary institutions. Journal of Eastern African Studies 4: 221-246. https://doi.org/10.1080/175310 $\underline{55.2010 .487333}$

Bassi M. \& Tache B. 2011. The community conserved landscape of the Borana Oromo, Ethiopia: opportunities and problems. Management of Environmental Quality 22: 174-186. https://doi. org/10.1108/14777831111113365

Benson C.W. 1945. Notes on the birds of Southern Abyssinia (Part 1). Ibis 86: 366-400. https://doi. org/10.1111/j.1474-919X.1945.tb01383.x

BirdLife International. 2008. Many bird species are confined to just one of the world's terrestrial biomes. Available from http://datazone.birdlife.org/sowb/casestudy/many-bird-species-are-confined-to-justone-of-the-world's-terrestrial-biomes [accessed 31 Jan. 2017] 
BirdLife South Africa 2014. White-winged Flufftail Conservation Report for African Bird Club 2014. Available from: https://www.africanbirdclub.org/sites/default/files/2013 White winged_Flufftail_ research_0.pdf [accessed 31 Jan. 2017]

BirdLife International 2017. Species factsheet: Spizocorys personata. Available from http://www. birdlife.org [accessed 7 Mar. 2017]

Bladon A.J., Töpfer T., Collar N.J., Gedeon K., Donald P.F., Dellelegn Y., Wondafrash M., Denge J., Dadacha G., Adula M. \& Green R.E. 2015. Notes on the behaviour, plumage and distribution of the White-tailed Swallow Hirundo megaensis. Bulletin of the African Bird Club 22: 148-161.

Bladon A.J., Jones S.E.I., Collar N.J., Dellelegn Y., Donald P.F., Gedeon K., Green R.E., Spottiswoode C.N., Töpfer T. \& Wondafrash M. 2016. Further notes on the natural history of the Ethiopian Bush-crow Zavattariornis stresemanni. Bulletin of the African Bird Club 23: 27-45.

Borghesio L. \& Massa R. 2000. Status and conservation of Prince Ruspoli's Turaco Tauraco ruspolii. Ostrich 71: 355-358. https://doi.org/10.1080/00306525.2000.9639958

Borghesio L. \& Giannetti F. 2005. Habitat degradation threatens the survival of the Ethiopian bush crow Zavattariornis stresemanni. Oryx 39: 44-49. https://doi.org/10.1017/S0030605305000086

Borrow N. 2010. Djibouti \& Somaliland. Saturday 4th September-Saturday 25th September 2010. Trip report. Available from: http://www.birdquest-tours.com/Djibouti-Somaliland-birding-tour-report/2010 [accessed 31 Jan. 2017]

Brown L.H., Urban E.K. \& Newman K. 1982. The Birds of Africa. Vol. 1. Ostriches to Birds of Prey. Academic Press. London.

Bulgarian Society for Protection of Birds. 2010. New expedition for the Egyptian Vultures in Ethiopia. Available from http://www.neophron.bspb.org/news-en/101222.html [accessed 25 Aug. 2015]

Central Statistical Agency of Ethiopia. 2012. 2011 National Statistics (Abstract). Available from http:// www.csa.gov.et. [accessed 25 Aug. 2015]

Clements J.F., Schulenberg T.S., Iliff M.J., Roberson D., Fredericks T.A., Sullivan B.L. \& Wood C. L. 2015. The eBird/Clements checklist of birds of the world: v2015. Available from http://www.birds. cornell.edu/clementschecklist/download/ [accessed 03 Feb. 2017]

Clouet M. \& Barrau C. 1999. The Golden Eagle, (Aquila chrysaetos) in the Bale Mountains, Ethiopia. J. Raptor Res. 22(2): 102-109.

Collar N.J. \& Stuart S.N. 1985. Threatened Birds of Africa and Related Islands: The ICBP/IUCN red data book, part 1. Third edition. International Council for Preservation and International Union for Conservation of Nature and Natural Resources, Cambridge.

Conover H.B. 1930. A new species of francolin from Southern Abyssinia. Proceedings of the Biological Society of Washington 43: 3-4.

Dessie G. \& Kleman J. 2007. Pattern and Magnitude of Deforestation in the South Central Rift Valley Region of Ethiopia. Mountain Research and Development 27: 162-168. https://doi.org/10.1659/ $\underline{\operatorname{mrd} .0730}$

Donald P.F., Buchanan G.M., Collar N.J., Dellelegn Abebe Y., Gabremichael M.N., Mwangi M.A.K., Ndang'ang'a P.K., Spottiswoode C.N. \& Wondafrash M. 2010. Rapid declines in habitat quality and population size of the Liben Lark Heteromirafra sidamoensis necessitate immediate conservation action. Bird Conservation International 20: 1-12. https://doi.org/10.1017/S0959270910000018 
Donald P.F., Gedeon K., Collar N.J., Spottiswoode C.N., Wondafrash M. \& Buchanan G.M. 2012. The restricted range of the Ethiopian Bush-crow Zavattariornis stresemanni is a consequence of high reliance on modified habitats within narrow climatic limits. Journal of Ornithology 153: 1031-1044. https://doi.org/10.1007/s10336-012-0832-4

Dowsett R.J. \& Dowsett-Lemaire F. 2015. New avian observations from south-western Ethiopia, with a review of overlooked literature and altitudinal limits. Bulletin of the British Ornithologists' Club 135: 224-239.

Elzen R. van den \& König C. 1983. Vögel des (Süd-) Sudan: taxonomische und tiergeographische Bemerkungen. Bonner zoologische Beiträge 34: 149-196.

Ethiopian Wildlife and Natural History Society. 2008/2009. Settlement expansion and natural resource management problems in the Abijiatta-Shalla Lakes National Park. Walia 26: 3-54.

Ethiopian Wildlife and Natural History Society (ed.) 2011. A Glimpse at Biodiversity Hotspots of Ethiopia. Ethiopian Wildlife and Natural History Society, Addis Ababa.

Fisher D., Gabremichael M. \& Yates P. 2012. First record of Arctic Tern Sterna paradisaea for Ethiopia. Bulletin of the African Bird Club 19: 211-212.

Fishpool L.D.C. \& Evans M.I. 2001. Important Bird Areas in Africa and Associated Islands: Priority sites for conservation. Pisces Publications and BirdLife International, Newbury and Cambridge. Available from http://datazone.birdlife.org/userfiles/file/IBAs/AfricaCntryPDFs/prelims.pdf [accessed 03 Feb. 2017]

Fry C.H., Keith S. \& Urban E.K. (eds) 1988. The Birds of Africa. Vol. 3.: Parrots to Woodpeckers. Academic Press, London.

Fry C.H., Keith S. \& Urban E.K. (eds) 2000. The Birds of Africa. Vol. 6.: Picathartes to Oxpeckers. Academic Press, London.

Fry C.H. \& Keith S. (eds) 2004. The Birds of Africa. Vol. 7.: Sparrows to Buntings. Christopher Helm, London.

Gabremichael M.N., Spottiswoode C.N., Fishpool L., Forsyth E., Lewis A., Pain D., Thomas R. \& Toye N. 2009. Occurrence of White- tailed Swallow Hirundo megaensis near Negele, Ethiopia. Bulletin of the African Bird Club 16: 83-86.

Gedeon K. 2006. Observations on the biology of the Ethiopian Bush Crow Zavattariornis stresemanni. Bulletin of the African Bird Club 13: 178-188.

Gedeon K., Cauldwell A., Ewnetu M., Regasa F., Schönbrodt R. \& Töpfer T. 2015. House Sparrow Passer domesticus and hybrids with Somali Sparrow Passer castanopterus in Ethiopia. Bulletin African Bird Club 22: 70-73.

Gelang M., Cibois A., Pasquet E., Olsson U., Alström P. \& Ericson P.G.P. 2009. Phylogeny of babblers (Aves, Passeriformes): major lineages, family limits and classification. Zoologica Scripta 38: 225-236. https://doi.org/10.1111/j.1463-6409.2008.00374.x

Gemedo-Dalle B., Maass L. \& Isselstein J. 2006. Rangeland condition and trend in the semi-arid Borana lowlands, southern Oromia, Ethiopia. African Journal of Range and Forage Science 23: 49-58. https:// doi.org/10.2989/10220110609485886

Heuglin T. von. 1856. Systematische Uebersicht der Vogel Nord-Ost-Afrika's mit Einschluss der arabischen Küste des Rothen Meeres u. der Nil-Quellen-Länder südwärts bis zum IV Gr. nördl. Breite. Sitzungsberichte der Kaiserlichen Akademie der Wissenschaften. Mathematisch-naturwissenschaftiche Classe 19: 255-324. 
Heuglin T. von. 1868. Reise nach Abessinien, den Gala-Ländern, Ost-Sudán und Chartúm in den Jahren 1861 und 1862. Costenoble, Jena.

Heuglin T. von. 1869-1874. Ornithologie Nordost-Afrikas, der Nilquellen und Küstengebiete des Rhoten Meeres und des nördlichen Somal-Landes. 4 Volumes. Theodor Fischer, Kassel.

Homann S. 2005. Indigenous Knowledge of Borana Pastoralists in Natural Resource Management: A case study from southern Ethiopia. Cuvillier, Göttingen.

Hoyo J. del \& Collar N.J. 2014. HBW and BirdLife International Illustrated Checklist of the Birds of the World. Vol. 1. Non-passerines. Lynx Edicions, Barcelona.

Hoyo J. del, Elliott A. \& Christie D.A. (eds) 2003. Handbook of the Birds of the World. Vol. 8. Lynx Edicions, Barcelona.

Hoyo J. del, Elliott A. \& Christie D.A. (eds) 2004. Handbook of the Birds of the World. Vol. 9. Lynx Edicions, Barcelona.

Hoyo J. del, Elliott A. \& Christie D.A. (eds) 2005. Handbook of the birds of the world. Vol. 10. Lynx Edicions, Barcelona.

Hoyo J. del, Elliott A. \& Christie D.A. (eds) 2006. Handbook of the Birds of the World. Vol. 11. Lynx Edicions, Barcelona.

Hoyo J. del, Elliott A. \& Christie D.A. (eds) 2007. Handbook of the Birds of the World. Vol. 12. Lynx Edicions, Barcelona.

Hoyo J. del, Elliott A. \& Christie D.A. (eds) 2008. Handbook of the Birds of the World. Vol. 13. Lynx Edicions, Barcelona.

Hoyo J. del, Elliott A. \& Christie D.A. (eds) 2009. Handbook of the Birds of the World. Vol. 14. Lynx Edicions, Barcelona.

Hoyo J. del, Elliott A. \& Christie D.A. (eds) 2010. Handbook of the Birds of the World. Vol. 15. Lynx Edicions, Barcelona.

Hoyo J. del, Elliott A. \& Christie D.A. (eds) 2011. Handbook of the Birds of the World. Vol. 16. Lynx Edicions, Barcelona.

Hoyo J. del, Elliott A. \& Sargatal J. (eds) 1992. Handbook of the Birds of the World. Vol. 1. Lynx Edicions, Barcelona.

Hoyo J. del, Elliott A. \& Sargatal J. (eds) 1994. Handbook of the Birds of the World. Vol. 2. Lynx Edicions, Barcelona.

Hoyo J. del, Elliott A. \& Sargatal J. (eds) 1996. Handbook of the Birds of the World. Vol. 3. Lynx Edicions, Barcelona.

Hoyo J. del, Elliott A. \& Sargatal J. (eds) 1997. Handbook of the Birds of the World. Vol. 4. Lynx Edicions, Barcelona.

Hoyo J. del, Elliott A. \& Sargatal J. (eds) 1999. Handbook of the Birds of the World. Vol. 5. Lynx Edicions, Barcelona.

Hoyo J. del, Elliott A. \& Sargatal J. (eds) 2001. Handbook of the Birds of the World. Vol. 6. Lynx Edicions, Barcelona.

Hoyo J. del, Elliott A. \& Sargatal J. (eds) 2002. Handbook of the Birds of the World. Vol. 7. Lynx Edicions, Barcelona. 
Jennings, M.C. 2010. Atlas of the Breeding Birds of Arabia. Fauna of Arabia 25: 1-751.

Keith S., Urban E.K. \& Fry C.H. (eds) 1992. The Birds of Africa. Vol. 4.: Broadbills to Chats. Academic Press, London.

Lewis A. \& Pomeroy D. 1989. A Bird Atlas of Kenya. Balkema, Rotterdam and Brookfield.

Linder H.P., de Klerk H.M., Born J., Fjeldså J. \& Rahbek C. 2012. The partitioning of Africa: statistically defined biogeographical regions in sub-Saharan Africa. Journal of Biogeography 39: 1189-1205. https:// doi.org/10.1111/j.1365-2699.2012.02728.x

Londei T. 2005. The hybrid population of Pied Crow Corvus albus and Somali Crow C. edithae on Dahlak Kebir Island, Eritrea: a case of assortative mating? Bulletin of the African Bird Club 12: 37-39, 98 (Corrigenda).

Londei T. 2008. The Pied Crow Corvus albus and Somali Crow Corvus edithae do not hybridise as soon as they meet. Scopus 27: 1-5.

Louette M. \& Borrow N. 2010. Plumage variation in African Goshawk Accipiter tachiro and its separation from congenerics in equatorial Africa. Bulletin of the African Bird Club 17: 19-31.

MapLibrary 2013. Ethiopia. Available from http://maplibrary.org/library/stacks/Africa/Ethiopia/index. $\underline{\mathrm{htm}}$ [accessed 25 Aug. 2015]

McSweeney C., New M \& Lizcano G. 2010. UNDP Climate Change Country Profiles: Ethiopia. Available from http://www.geog.ox.ac.uk/research/climate/projects/undp-cp/

Mellanby R.J., Ross B., Watt A., Wondafrash M., Ewnetu M., Broadhurst C., Critchlow R., Dadesa A., Deas T., Enawgaw C., Gebremedhin B., Graham E., Maclean S., McKean M., Collar N.J. \& Spottiswoode C.N. 2008. Distribution, abundance and habitat preferences of White-tailed Swallow Hirundo megaensis and Ethiopian Bush-crow Zavattariornis stresemanni, two southern Ethiopian endemics. Bird Conservation International 18: 395-412. https://doi.org/10.1017/S0959270908007314

Mills M.S.L. \& Cohen C. 2015. Are Yellow-throated Seedeater Crithagra flavigula and White-throated Seedeater C. xanthopygia conspecific? Observations of seedeaters from Djibouti. Bulletin of the African Bird Club 22: 190-195.

Moltoni E. \& Ruscone G. 1940-1944. Gli uccelli dell'Africa Orientale Italiana. Vols 1-4. Premiata Tipografia Successori Fratelle Fusi, Pavia.

Nikolaus G. 1987. Distribution atlas of Sudan's birds with notes on habitat and status. Bonner zoologische Monographien 25: 1-322.

Otieno P.O., Lalah J.O., Virani M., Jondiko I.O. \& Schramm K.W. 2011. Carbofuran use and abuse in Kenya: residues in soils, plants, water courses and the African white-backed vultures (Gyps africanus) found dead. The Environmentalist 31: 382-393.

Rannestad O.T. 2016. Additions to the Ethiopian bird atlas: 126 new records from 14 atlas squares. Scopus 36: 1-14.

Redman N. 2012. Djibouti \& Somaliland. Sunday 9th September - Thursday 27th September 2012. Available from http://www.birdquest-tours.com/Djibouti-Somaliland-birding-tour-report/j

Redman N., Stevenson T. \& Fanshawe J. 2011. Birds of the Horn of Africa: Ethiopia, Eritrea, Djibouti, Somalia and Socotra. Christopher Helm, London.

Reichenow A. 1901-1905. Die Vögel Afrikas. Vols 1-3. Neumann, Neudamm. 
Ross B., Wondafrash M., Ewnetu M., Watt S., Broadhurst C., Critchlow R., Dadesa A., Deas T., Enawgaw C., Gebremedhim B., Graham E., Maclean S. \& Mellanby R.J. 2009. Notes on the ecology of Ethiopian Bush-crow Zavattariornis stresemanni. Scopus 29: 1-6.

Rüppell E. 1845. Systematische Uebersicht der Vögel Nord-Ost-Afrika's nebst Abbildung und Beschreibung von fünfzig theils unbekannten, theils noch nicht bildlich dargestellten Arten. Schmerber, Frankfurt am Main.

Safford R.J., Ash J.S., Duckworth J.W., Telfer M.G. \& Zewdie C. 1995. A new species of nightjar from Ethiopia. Ibis 137: 301-307. https://doi.org/10.1111/j.1474-919X.1995.tb08025.X

Shelley G.E. 1896-1912. The birds of Africa. Comprising all the species which occur in the Ethiopian Region. 5 Volumes. R. H. Porter, London. https://doi.org/10.5962/bhl.title.14123

Smallie J. \& Virani M.Z. 2010. A preliminary assessment of the potential risks from electrical infrastructure to large birds in Kenya. Scopus 30: 32-39.

Spottiswoode C.N., Wondafrash M., Gabremichael M.N., Delelegn Abebe Y., Mwangi M.A.K., Collar N.J. \& Dolman P.M. 2009. Rangeland degradation is poised to cause Africa's first recorded avian extinction. Animal Conservation 12: 249-257.

Spottiswoode C.N., Olsson U., Mills M.S.L., Cohen C., Francis J.E., Toye N., Hoddinott D., Dagne A., Wood C., Donald P.F., Collar N.J. \& Alström P. 2013. Rediscovery of a long-lost lark reveals the conspecificity of endangered Heteromirafra populations in the Horn of Africa. Journal of Ornithology 154: 813-825. https://doi.org/10.1007/s10336-013-0948-1

Stattersfield A.J., Crosby M.J., Long A.J. \& Wege D.C. 1998. Endemic Bird Areas of the World: Priorities for biodiversity conservation. BirdLife International, Cambridge.

Takahashi R. \& Todo Y. 2012. Impact of Community-Based Forest Management on Forest Protection: Evidence from an Aid-Funded Project in Ethiopia. Environmental management 50: 396-404. https://doi. org/10.1007/s00267-012-9887-5

Tietze D.T. \& Martens J. 2010. Intraspecific differentiation in Spotted Creepers Salpornis spilonotus (Aves: Passeriformes: Certhiidae). Vertebrate Zoology 60 :163-170.

Töpfer T. \& Gedeon K. 2012. The construction and thermal insulation of Ethiopian Bush-crow (Zavattariornis stresemanni) nests: a preliminary study. Avian Biology Research 5: 198-202. https://doi. org/10.3184/175815512X13534399582256

Töpfer T., Podsiadlowski L. \& Gedeon K. 2014. Rediscovery of the Black-fronted Francolin Pternistis (castaneicollis) atrifrons (Conover, 1930) (Aves: Galliformes: Phasianidae) with notes on biology, taxonomy and conservation. Vertebrate Zoology 64: 261-271.

Urban E.K. \& Brown L.H.A 1991. Checklist of the Birds of Ethiopia. Haile Sellassie I University. Addis Abeba.

Urban E.K., Fry C.H. \& Keith S. (eds) 1986. The Birds of Africa. Vol. 2: Game Birds to Pigeons. Academic Press, London.

Urban E.K., Fry C.H. \& Keith S. (eds) 1997. The Birds of Africa. Vol. 5: Thrushes to Puffback Flycatchers. Academic Press, London.

Virani M.Z., Kendall C., Njoroge P. \& Thomsett S. 2011. Major declines in the abundance of vultures and other scavenging raptors in and around the Masai Mara ecosystem, Kenya. Biological Conservation 144: 746-752. https://doi.org/10.1016/j.biocon.2010.10.024 
Vreugdenhil D., Vreugdenhil A., Tilahun T., Shimelis A. \& Tefera Z. (With technical contributions from L. Nagelkerke, K. Gedeon, S. Spawls, D. Yalden, L. Berhanu \& L. Siege.) 2012. Gap Analysis of the Protected Areas System of Ethiopia. World Institute for Conservation and Environment and Ethiopian Wildlife Conservation Authority, Addis Ababa. Available from http://www.ewca.gov.et/sites/default/ files/gap_analysis_of the_protected_areas_system_of_ethiopia_part_1.pdf [accessed 06 Mar. 2017]

Wink M., Clouet M., Goar J.-L. \& Barrau C. 2004. Sequence variation in the cytochrome b gene of subspecies of Golden Eagles Aquila chrysaetos. Alauda 72: 153-157.

Manuscript received: 6 November 2015

Manuscript accepted: 8 August 2016

Published on: 30 March 2017

Topic editor: Rudy Jocqué

Section editor: Michel Louette

Desk editor: Jeroen Venderickx

Printed versions of all papers are also deposited in the libraries of the institutes that are members of the EJT consortium: Muséum national d'Histoire naturelle, Paris, France; Botanic Garden Meise, Belgium; Royal Museum for Central Africa, Tervuren, Belgium; Natural History Museum, London, United Kingdom; Royal Belgian Institute of Natural Sciences, Brussels, Belgium; Natural History Museum of Denmark, Copenhagen, Denmark; Naturalis Biodiversity Center, Leiden, the Netherlands. 
Appendix 1. Checklist of the birds of Oromia, Ethiopia. Subspecies: (number of subspecies found in Oromia / total number of subspecies per species): $\mathrm{n}=$ only nominate form in Oromia; $\mathrm{m}=$ monotypic; $*=$ further comments in appendix 2 . Status of occurrence: $\mathrm{R}=$ all year resident; $\mathrm{Af}=$ Afrotropical migrant or nomad; $\mathrm{Pl}$ = Palearctic migrant; $(\mathrm{B})$ = breeding confirmed in Oromia; (b) = breeding assumed; $?=$ status questionable. Abundance: $*=$ vagrant or rare; $* *=$ uncommon; $* * *=$ common. Endemism: $\mathrm{O}=$ Oromia; $\mathrm{E}=$ Ethiopia; $\mathrm{H}=$ Horn of Africa (incl. Ethiopia, Eritrea, Somalia, Djibouti and Socotra). $\mathrm{H}+=$ Horn of Africa incl. cases where offshoots of the ranges extend to Kenya, Sudan and South Suda. Biome: $\mathrm{SG}=$ Sudan-Guinea; $\mathrm{SM}=$ Somali-Masai; $\mathrm{AH}=$ Afrotropical Highlands. Conservation status: $\mathrm{CR}=$ critically endangered; $\mathrm{EN}=$ endangered; $\mathrm{VU}=$ vulnerable; $\mathrm{NT}=$ near threatened (according to IUCN Red List of Threatened Species, version 2012.1). Official protection/ regulations: CMS = Convention on the Conservation of Migratory Species of Wild Animals; AEWA = Agreement on the Conservation of African-Eurasian Migratory Waterbirds; CITES = Convention on International Trade in Endangered Species of Wild Fauna and Flora; $\mathrm{P}=$ protected; $\mathrm{T}=$ tradable; $\mathrm{H}=$ huntable according to regional regulations. The English and scientific names follow Clements et al. (2015). Taxon numbering, separated by slash, corresponds to the numbers given by Clements et al. (2015) and Ash \& Atkins (2009), respectively.

\begin{tabular}{|c|c|c|c|c|c|c|c|c|}
\hline 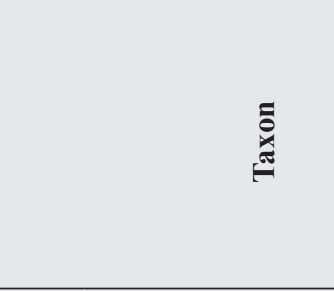 & 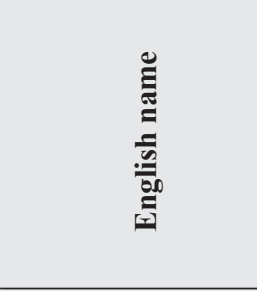 & 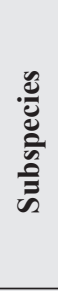 & 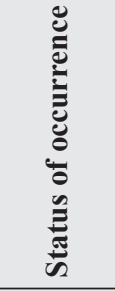 & 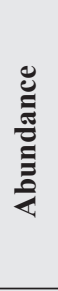 & 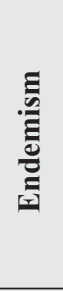 & $\begin{array}{l}\stackrel{0}{\Xi} \\
\text { है }\end{array}$ & 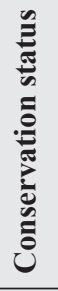 & 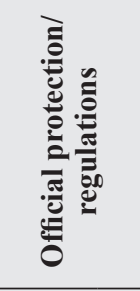 \\
\hline \multicolumn{9}{|c|}{ STRUTHIONIFORMES: Struthionidae } \\
\hline $\begin{array}{l}1 / 1 \text { Struthio camelus } \\
\text { Linnaeus, } 1758\end{array}$ & Common Ostrich & $1 / 4^{*}$ & $\mathrm{R}(\mathrm{B})$ & $* *$ & & & & $\mathrm{H}$ \\
\hline $\begin{array}{l}\text { 6/1 } \begin{array}{l}\text { Struthio molypdophanes } \\
\text { Reichenow, } 1882\end{array}\end{array}$ & Somali Ostrich & $\mathrm{m}^{*}$ & $\mathrm{R}(\mathrm{B})$ & $* *$ & & & & $\mathrm{H}$ \\
\hline \multicolumn{9}{|c|}{ ANSERIFORMES: Anatidae } \\
\hline $\begin{array}{c}216 / 17 \begin{array}{l}\text { Dendrocygna viduata } \\
\text { (Linnaeus, 1766) }\end{array}\end{array}$ & $\begin{array}{l}\text { White-faced Whistling- } \\
\text { Duck }\end{array}$ & $\mathrm{m}$ & $\mathrm{R}(\mathrm{B})+\mathrm{Af}$ & $* * *$ & & & & AEWA; T, H \\
\hline $\begin{array}{l}223 / 18 \begin{array}{l}\text { Dendrocygna bicolor } \\
\text { (Vieillot, 1816) }\end{array}\end{array}$ & Fulvous Whistling-Duck & $\mathrm{m}$ & $\operatorname{Af}(b)$ & $* *$ & & & & AEWA; T, H \\
\hline $\begin{array}{l}230 / 19 \begin{array}{l}\text { Thalassornis leuconotus } \\
\text { Eyton, } 1838\end{array}\end{array}$ & White-backed Duck & $\mathrm{n} / 2$ & $\mathrm{R}(\mathrm{b})$ & $* *$ & & & & AEWA; T, H \\
\hline $\begin{array}{c}321 / 22 \begin{array}{l}\text { Cyanochen cyanoptera } \\
\text { (Rüppell, 1845) }\end{array}\end{array}$ & Blue-winged Goose & $\mathrm{m}$ & $\mathrm{R}(\mathrm{B})$ & $* * *$ & E, $\mathrm{H}$ & $\mathrm{AH}$ & VU & \\
\hline 337/21 $\begin{array}{l}\text { Sarkidiornis melanotos } \\
\text { (Pennant, 1769) }\end{array}$ & Comb Duck & $\mathrm{n} / 2$ & Af & $* * *$ & & & & AEWA; T, H \\
\hline $\begin{array}{c}353 / 23 \begin{array}{l}\text { Alopochen aegyptiaca } \\
\text { (Linnaeus, 1766) }\end{array}\end{array}$ & Egyptian Goose & $\mathrm{m}$ & $\mathrm{R}(\mathrm{B})$ & $* * *$ & & & & AEWA; T, H \\
\hline $\begin{array}{l}354 / 25 \begin{array}{l}\text { Tadorna ferruginea } \\
\text { (Pallas, 1764) }\end{array}\end{array}$ & Ruddy Shelduck & $\mathrm{m}^{*}$ & $\mathrm{R}(\mathrm{B})+\mathrm{Pl} ?$ & $* *$ & & & & AEWA \\
\hline $\begin{array}{l}358 / 24 \begin{array}{l}\text { Tadorna tadorna } \\
\text { (Linnaeus, 1758) }\end{array}\end{array}$ & Common Shelduck & $\mathrm{m}$ & $\mathrm{Pl}$ & $*$ & & & & AEWA \\
\hline $\begin{array}{l}363 / 20 \begin{array}{l}\text { Plectropterus gambensis } \\
\text { (Linnaeus, 1766) }\end{array}\end{array}$ & Spur-winged Goose & $\mathrm{n} / 2$ & $\mathrm{R}(\mathrm{B})+\mathrm{Af} ?$ & $* * *$ & & & & AEWA; T, H \\
\hline $383 / 26 \begin{array}{l}\text { Nettapus auritus } \\
\text { (Boddaert, 1783) }\end{array}$ & African Pygmy-Goose & $\mathrm{m}$ & $\mathrm{R} ?+\mathrm{Af}$ & $* *$ & & & & AEWA \\
\hline $\begin{array}{l}400 / 30 \text { Anas sparsa } \\
\text { Eyton, } 1838\end{array}$ & African Black Duck & $1 / 2^{*}$ & $\mathrm{R}(\mathrm{B})$ & $* * *$ & & & & $\mathrm{~T}, \mathrm{H}$ \\
\hline $\begin{array}{l}\text { 403/28 Anas strepera } \\
\text { Linnaeus, } 1758\end{array}$ & Gadwall & $\mathrm{n} / 2$ & $\mathrm{Pl}$ & $* *$ & & & & AEWA; T, H \\
\hline $\begin{array}{c}\text { 407/29 Anas penelope } \\
\text { Linnaeus, } 1758\end{array}$ & Eurasian Wigeon & $\mathrm{m}$ & $\mathrm{Pl}$ & $* * *$ & & & & AEWA; T, H \\
\hline $\begin{array}{l}\text { 415/32 Anas undulata } \\
\text { C.F. Dubois, } 1839\end{array}$ & Yellow-billed Duck & $1 / 2^{*}$ & $\mathrm{R}(\mathrm{B})$ & $* * *$ & & & & AEWA; T, H \\
\hline $\begin{array}{l}\text { 419/31 Anas platyrhynchos } \\
\text { Linnaeus, } 1758\end{array}$ & Mallard & $\mathrm{n} / 2$ & $\mathrm{Pl}$ & $*$ & & & & AEWA; T, H \\
\hline $\begin{array}{l}\text { 466/33 Anas clypeata } \\
\text { Linnaeus, } 1758\end{array}$ & Northern Shoveler & $\mathrm{m}$ & $\mathrm{Pl}$ & $* * *$ & & & & AEWA; T, H \\
\hline $\begin{array}{c}\text { 478/34 Anas erythrorhyncha } \\
\text { Gmelin, } 1789\end{array}$ & Red-billed Duck & $\mathrm{m}$ & $\mathrm{R}(\mathrm{B})+\mathrm{Af} ?$ & $* *$ & & & & AEWA; T, H \\
\hline $\begin{array}{l}\text { 479/35 Anas acuta } \\
\text { Linnaeus, } 1758\end{array}$ & Northern Pintail & $\mathrm{m}$ & $\mathrm{Pl}$ & $* * *$ & & & & AEWA; T, H \\
\hline
\end{tabular}




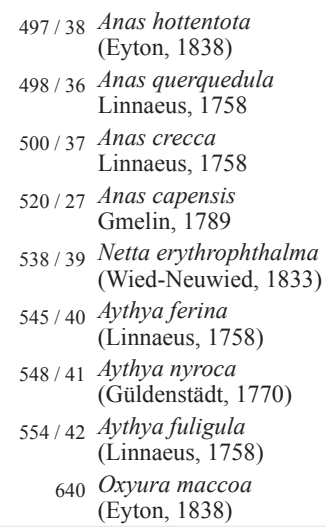

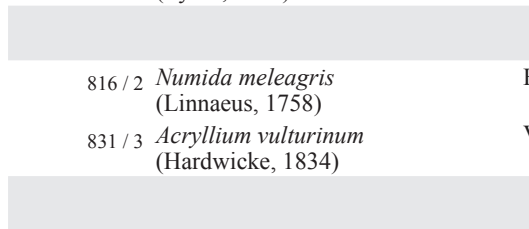
Ptilopachus petrosus (Gmelin, 1789)

1149 / 15 Coturnix coturnix (Linnaeus, 1758)

1150 / 16 Coturnix delegorguei Delegorgue, 1847

1228 / 12 Pternistis castaneicollis (Salvadori, 1888)

$1231 / 13$ Pternistis erckelii (Rüppell, 1835)

1239/9 Pternistis squamatus (Cassin, 1857)

1246 / 10 Pternistis clappertoni (Children \& Vigors, 1826)

1247 / 11 Pternistis harwoodi (Blundell \& Lovat, 1899)

1251 / 14 Pternistis leucoscepus G. (R. Gray, 1867)

1282 / 8 Francolinus sephaena (A. Smith, 1836)

1304 / 5 Peliperdix coqui (A. Smith, 1836)

1324/ 7 Scleroptila gutturalis (Rüppell, 1835)

1331/ 6 Scleroptila psilolaema (G. R. Gray, 1867)

$\begin{aligned} & 1676 / 47 \begin{array}{l}\text { Tachybaptus ruficollis } \\ \text { (Pallas, 1764) }\end{array} \\ & 1719 / 48 \begin{array}{l}\text { Podiceps cristatus } \\ \text { (Linnaeus, 1758) }\end{array} \\ & 1723 / 49 \begin{array}{l}\text { Podiceps nigricollis } \\ \text { C. L. Brehm, 1831 }\end{array} \\ &\end{aligned}$

Hottentot Teal
Garganey
Green-winged Teal
Cape Teal
Southern Pochard
Common Pochard
Ferruginous Duck
Tufted Duck
Maccoa Duck

$\begin{array}{ccc}\mathrm{m} & \text { Af } & * * \\ \mathrm{~m} & \mathrm{Pl} & * * * \\ \mathrm{n} / 2 & \mathrm{Pl} & * * * \\ \mathrm{~m} & \mathrm{R}(\mathrm{B}) & * * * \\ 1 / 2 * & \mathrm{Af} & * * \\ \mathrm{~m} & \mathrm{Pl} & * * \\ \mathrm{~m} & \mathrm{Pl} & * * \\ \mathrm{~m} & \mathrm{Pl} & * * \\ \mathrm{~m} & \mathrm{R}(\mathrm{b}) & * * *\end{array}$

AEWA; T, H

AEWA; T, H

AEWA; T, H

AEWA; T, H

AEWA; T, H

AEWA; T, H

GALLIFORMES: Numididae

Helmeted Guineafowl $\quad 2 / 9 * \quad \mathrm{R}(\mathrm{B})$

$* * *$

$\mathrm{T}, \mathrm{H}$

$\begin{array}{lll}\text { Vulturine Guineafowl } & \mathrm{m} & \mathrm{R}(\mathrm{B})\end{array}$

SM

$\mathrm{T}$

\section{GALLIFORMES: Phasianidae}

Stone Partridge $1 / 2 * \quad R(B)$

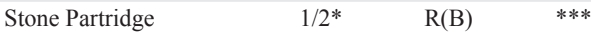

** T, H

Common Quail

$\mathrm{m}^{*}$

$\mathrm{Af}(\mathrm{B})+\mathrm{Pl}$

$\mathrm{T}, \mathrm{H}$

Harlequin Quail

$\mathrm{n} / 3$

$\mathrm{R}(\mathrm{B})+\mathrm{Af}$

T, H

Chestnut-naped Francolin $\quad 2 / 2 *$

$\mathrm{R}(\mathrm{B})$

**

T,

Erckel's Francolin

$\mathrm{R}(\mathrm{B})$

$* * *$

$\mathrm{H}+\quad \mathrm{AH}$

T, H

Scaly Francolin

$\mathrm{m}$

$\mathrm{H}+\quad \mathrm{AH}$

T, H

Clapperton's Francolin

$\mathrm{m}^{*}$

$\mathrm{R}(\mathrm{B})$

T, H

Clapperton's Francolin $\quad \mathrm{m}^{*}$

$\mathrm{R}(\mathrm{B})$

T, H

Harwood's Francolin

$\mathrm{m}$

$\mathrm{R}(\mathrm{B})$

.

E, $\mathrm{H} \quad \mathrm{AH} \quad \mathrm{VU} \quad \mathrm{P}$

Yellow-necked Francolin

Crested Francolin

$\mathrm{R}(\mathrm{B})$

$* * *$

SM

T, H

Coqui Francolin

$1 / 5^{*}$

$\mathrm{R}(\mathrm{B})$

$* * *$

T, H

$\mathrm{T}, \mathrm{H}$

Orange River Francolin $\quad 1 / 2 *$

$\mathrm{R}(\mathrm{B})$

T, H

Moorland Francolin

$\mathrm{n} / 2 *$

$\mathrm{R}(\mathrm{B})$

$* *$

T, H

PODICIPEDIFORMES: Podicipedidae

Little Grebe $\quad 1 / 9^{*} \quad \mathrm{R}(\mathrm{B})+\mathrm{Af} ?+\quad * * *$

$\begin{array}{ccc}\text { Great Crested Grebe } & \text { Pl? } \\ & 1 / 3 * & \mathrm{R}(\mathrm{B})+\mathrm{Af} ?+ \\ \mathrm{Pl} ?\end{array} \quad * * *$

AEWA

Eared Grebe

$\begin{array}{cc}1 / 3^{*} & \mathrm{R}(\mathrm{b})+\mathrm{Af} ?+ \\ \mathrm{Pl} ?\end{array}$

AEWA

AEWA

PHOENICOPTERIFORMES: Phoenicopteridae

1746/50 Phoenicopterus roseus Pallas, 1811

Greater Flamingo $\quad \mathrm{m} \quad \mathrm{R}+\mathrm{Af}+\mathrm{Pl} \quad * * *$

AEWA

Phoeniconaias minor

Lesser Flamingo

m $\quad \mathrm{R}(\mathrm{B})+\mathrm{Af}$

NT

AEWA

Saint-Hilaire, 1798)

\section{CICONIIFORMES: Ciconiidae}

2072 / 53 Anastomus lamelligerus

African Openbill

$\mathrm{n} / 2$

$\mathrm{R}(\mathrm{b})+\mathrm{Af}$

Temminck, 1823

$2075 / 54 \begin{aligned} & \text { Ciconia nigra } \\ & \text { (Linnaeus, 1758) }\end{aligned}$

Black Stork

$\mathrm{n} / 2$

(b) + Af

AEWA

(Linnaeus, 1758)

2076 / 55 Ciconia abdimii

Lichtenstein, 1823 2077 / $56 \begin{aligned} & \text { Ciconia episcopus } \\ & \text { (Boddaert, 1783) }\end{aligned}$

2084 / 57 Ciconia ciconia (Linnaeus, 1758)

\section{Abdim's Stork}

Woolly-necked Stork

m

$\mathrm{R}(\mathrm{B})+\mathrm{Af}$

R(b) + Af

White Stork

$\mathrm{n} / 2$

Pl 


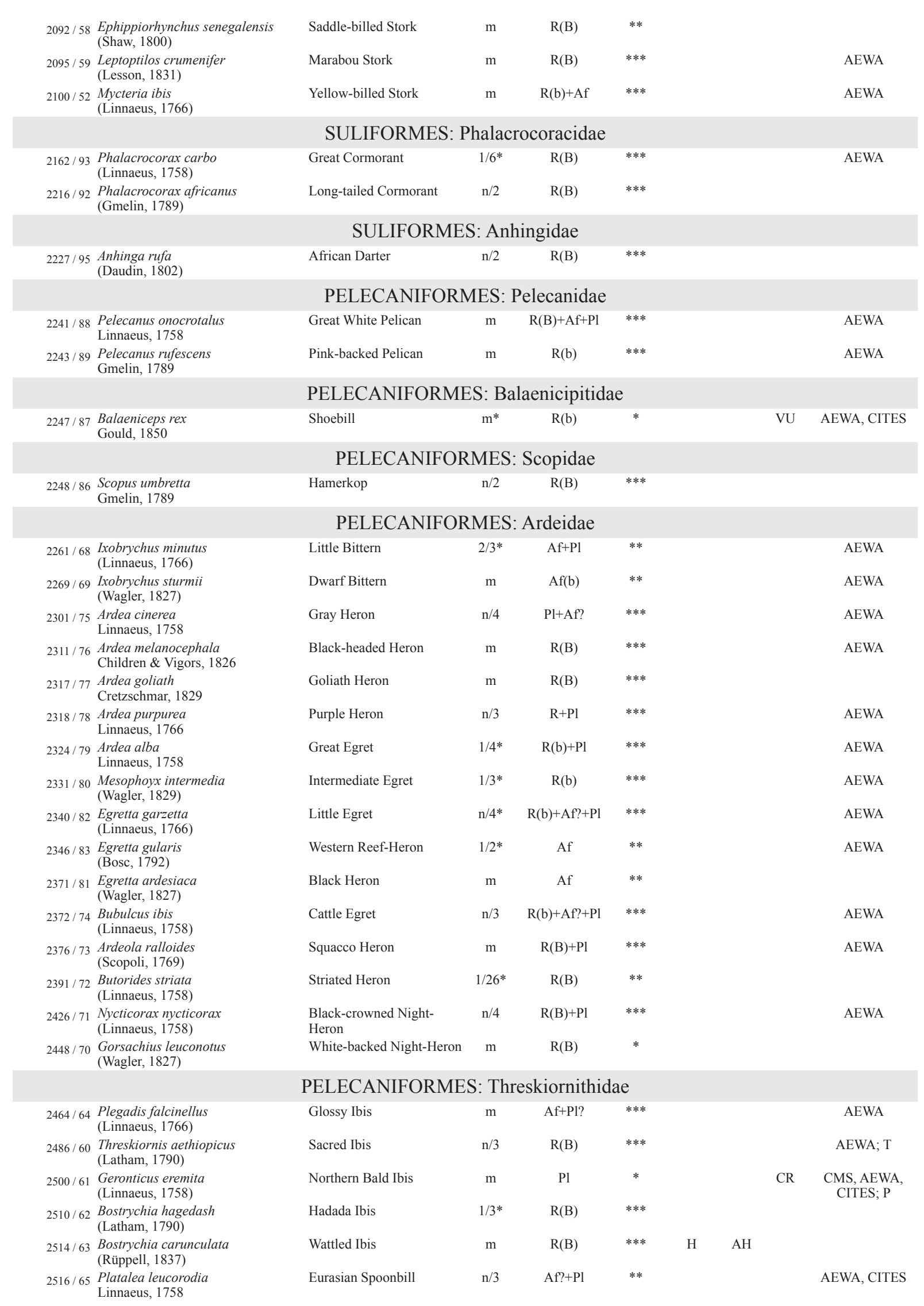




\begin{tabular}{|c|c|c|c|c|c|c|c|}
\hline $2521 / 66$ & $\begin{array}{l}\text { Platalea alba } \\
\text { Scopoli, } 1786\end{array}$ & African Spoonbill & $\mathrm{m}$ & $\mathrm{R}(\mathrm{b})$ & $* * *$ & & AEWA \\
\hline \multicolumn{8}{|c|}{ ACCIPITRIFORMES: Sagittariidae } \\
\hline $2544 / 114$ & $\begin{array}{l}\text { Sagittarius serpentarius } \\
\text { (J. F. Miller, 1779) }\end{array}$ & Secretarybird & $\mathrm{m}$ & $\mathrm{R}(\mathrm{B})$ & $* *$ & VU & CITES \\
\hline \multicolumn{8}{|c|}{ ACCIPITRIFORMES: Pandionidae } \\
\hline $2545 / 115$ & $\begin{array}{l}\text { Pandion haliaetus } \\
\text { (Linnaeus, 1758) }\end{array}$ & Osprey & $\mathrm{n} / 4$ & Af?+Pl & $* *$ & & CITES \\
\hline \multicolumn{8}{|c|}{ ACCIPITRIFORMES: Accipitridae } \\
\hline $2554 / 119$ & $\begin{array}{l}\text { Elanus caeruleus } \\
\text { (Desfontaines, 1789) }\end{array}$ & Black-shouldered Kite & $\mathrm{n} / 4$ & $\mathrm{R}(\mathrm{B})$ & $* *$ & & CITES \\
\hline $2564 / 120$ & $\begin{array}{l}\text { Chelictinia riocourii } \\
\text { (Vieillot, 1822) }\end{array}$ & Scissor-tailed Kite & $\mathrm{m}$ & $\mathrm{Af}(\mathrm{B})$ & $* *$ & & CITES \\
\hline $2565 / 140$ & $\begin{array}{l}\text { Polyboroides typus } \\
\text { A. Smith, } 1829\end{array}$ & African Harrier-Hawk & $\mathrm{n} / 2$ & $\mathrm{R}(\mathrm{B})$ & $* * *$ & & CITES \\
\hline $2571 / 123$ & $\begin{array}{l}\text { Gypaetus barbatus } \\
\text { (Linnaeus, 1758) }\end{array}$ & Lammergeier & $1 / 2^{*}$ & $\mathrm{R}(\mathrm{B})$ & $* *$ & & CITES; P \\
\hline $2574 / 124$ & $\begin{array}{l}\text { Neophron percnopterus } \\
\text { (Linnaeus, 1758) }\end{array}$ & Egyptian Vulture & $\mathrm{n} / 3^{*}$ & $\begin{array}{l}\mathrm{R}(\mathrm{B})+\mathrm{Af} ?+ \\
\quad \mathrm{Pl} ?\end{array}$ & $* *$ & EN & CMS, CITES \\
\hline 2586117 & $\begin{array}{l}\text { Pernis apivorus } \\
\text { (Linnaeus, 1758) }\end{array}$ & European Honey-Buzzard & $\mathrm{m}$ & Pl & $* *$ & & CITES \\
\hline $2607 / 116$ & $\begin{array}{l}\text { Aviceda cuculoides } \\
\text { Swainson, } 1837\end{array}$ & African Cuckoo-Hawk & $1 / 3^{*}$ & $\mathrm{R}(\mathrm{b})$ & $* *$ & & CITES \\
\hline $2638 / 129$ & $\begin{array}{l}\text { Trigonoceps occipitalis } \\
\text { (Burchell, 1824) }\end{array}$ & White-headed Vulture & $\mathrm{m}$ & $\mathrm{R}(\mathrm{B})$ & $* *$ & VU & CITES \\
\hline $2640 / 130$ & $\begin{array}{l}\text { Torgos tracheliotos } \\
\text { (J. R. Forster, 1796) }\end{array}$ & Lappet-faced Vulture & $1 / 3^{*}$ & $\mathrm{R}(\mathrm{B})$ & $* *$ & VU & CITES \\
\hline $2644 / 125$ & $\begin{array}{l}\text { Necrosyrtes monachus } \\
\text { (Temminck, 1823) }\end{array}$ & Hooded Vulture & $\mathrm{m}$ & $\mathrm{R}(\mathrm{B})$ & $* * *$ & EN & CITES \\
\hline $2645 / 126$ & $\begin{array}{l}\text { Gyps africanus } \\
\text { Salvadori, } 1865\end{array}$ & White-backed Vulture & $\mathrm{m}$ & $\mathrm{R}(\mathrm{B})$ & $* * *$ & EN & CITES \\
\hline $2649 / 127$ & $\begin{array}{l}\text { Gyps rueppelli } \\
\text { (A. E. Brehm, 1852) }\end{array}$ & Rueppell's Griffon & $1 / 2 *$ & $\mathrm{R}(\mathrm{B})$ & $* * *$ & EN & CITES \\
\hline $2654 / 128$ & $\begin{array}{l}\text { Gyps fulvus } \\
\text { (Hablizl, 1783) }\end{array}$ & Eurasian Griffon & $\mathrm{n} / 2$ & $\mathrm{Pl}$ & $* *$ & & CITES \\
\hline $2693 / 135$ & $\begin{array}{l}\text { Terathopius ecaudatus } \\
\text { (Daudin, 1800) }\end{array}$ & Bateleur & $\mathrm{m}$ & $\mathrm{R}(\mathrm{B})$ & $* * *$ & NT & CITES \\
\hline $2697 / 131$ & $\begin{array}{l}\text { Circaetus gallicus } \\
\text { (Gmelin, 1788) }\end{array}$ & Short-toed Eagle & $\mathrm{m}$ & Pl & $* *$ & & CITES \\
\hline $2699 / 132$ & $\begin{array}{l}\text { Circaetus pectoralis } \\
\text { A. Smith, } 1829\end{array}$ & $\begin{array}{l}\text { Black-breasted Snake- } \\
\text { Eagle }\end{array}$ & $\mathrm{m}$ & $\mathrm{R}(\mathrm{B})$ & $* *$ & & CITES \\
\hline $2700 / 133$ & $\begin{array}{l}\text { Circaetus cinereus } \\
\text { Vieillot, } 1818\end{array}$ & Brown Snake-Eagle & $\mathrm{m}$ & $\mathrm{R}(\mathrm{b})$ & $* *$ & & CITES \\
\hline $2702 / 134$ & $\begin{array}{l}\text { Circaetus cinerascens } \\
\text { J. W. von Müller, } 1851\end{array}$ & Banded Snake-Eagle & $\mathrm{m}$ & $\mathrm{R}(\mathrm{b})$ & $* *$ & & CITES \\
\hline $2704 / 118$ & $\begin{array}{l}\text { Macheiramphus alcinus } \\
\text { Westerman, } 1851\end{array}$ & Bat Hawk & $1 / 3^{*}$ & $\mathrm{R}(\mathrm{b})$ & $* *$ & & CITES \\
\hline $2711 / 172$ & $\begin{array}{l}\text { Stephanoaetus coronatus } \\
\text { (Linnaeus, 1766) }\end{array}$ & Crowned Hawk-Eagle & $\mathrm{m}$ & $\mathrm{R}(\mathrm{B})$ & $* *$ & NT & CITES \\
\hline $2734 / 170$ & $\begin{array}{l}\text { Polemaetus bellicosus } \\
\text { (Daudin, 1800) }\end{array}$ & Martial Eagle & $\mathrm{m}$ & $\mathrm{R}(\mathrm{B})$ & $* *$ & NT & CITES \\
\hline $2747 / 171$ & $\begin{array}{l}\text { Lophaetus occipitalis } \\
\text { (Daudin, 1800) }\end{array}$ & Long-crested Eagle & $\mathrm{m}$ & $\mathrm{R}(\mathrm{B})$ & $* * *$ & & CITES \\
\hline $2751 / 159$ & $\begin{array}{l}\text { Clanga pomarina } \\
\text { (C. L. Brehm, 1831) }\end{array}$ & Lesser Spotted Eagle & $\mathrm{m}$ & $\mathrm{Pl}$ & $* *$ & & CITES \\
\hline $2753 / 160$ & $\begin{array}{l}\text { Clanga clanga } \\
\text { (Pallas, 1811) }\end{array}$ & Greater Spotted Eagle & $\mathrm{m}$ & $\mathrm{Pl}$ & $* *$ & VU & CMS, CITES; P \\
\hline $2756 / 166$ & $\begin{array}{l}\text { Hieraaetus wahlbergi } \\
\text { (Sundevall, 1850) }\end{array}$ & Wahlberg's Eagle & $\mathrm{m}$ & $\mathrm{Af}(\mathrm{B})+\mathrm{R} ?$ & $* *$ & & CITES \\
\hline $2757 / 168$ & $\begin{array}{l}\text { Hieraaetus pennatus } \\
\text { (Gmelin, 1788) }\end{array}$ & Booted Eagle & $\mathrm{m}$ & $\mathrm{Pl}$ & $* *$ & & CITES \\
\hline $2760 / 169$ & $\begin{array}{l}\text { Hieraaetus ayresii } \\
\text { (Gurney, 1862) }\end{array}$ & Ayres's Hawk-Eagle & $\mathrm{m}$ & $\mathrm{R}(\mathrm{b})$ & $* *$ & & CITES \\
\hline $2761 / 161$ & $\begin{array}{l}\text { Aquila rapax } \\
\text { (Temminck, 1828) }\end{array}$ & Tawny Eagle & $1 / 3^{*}$ & $\mathrm{R}(\mathrm{B})$ & $* * *$ & & CITES \\
\hline $2765 / 162$ & $\begin{array}{l}\text { Aquila nipalensis } \\
\text { Hodgson, } 1833\end{array}$ & Steppe Eagle & $1 / 2 *$ & $\mathrm{Pl}$ & $* * *$ & & CITES \\
\hline $2769 / 163$ & $\begin{array}{l}\text { Aquila heliaca } \\
\text { Savigny, } 1809\end{array}$ & Imperial Eagle & $\mathrm{m}$ & Pl & $*$ & VU & CMS, CITES; P \\
\hline $2771 / 164$ & $\begin{array}{l}\text { Aquila chrysaetos } \\
\text { (Linnaeus, 1758) }\end{array}$ & Golden Eagle & $1 / 6^{*}$ & $\mathrm{R}(\mathrm{B})$ & $*$ & & CITES \\
\hline $2782 / 165$ & $\begin{array}{l}\text { Aquila verreauxii } \\
\text { Lesson, } 1831\end{array}$ & Verreaux's Eagle & $\mathrm{m}$ & $\mathrm{R}(\mathrm{B})$ & $* *$ & & CITES \\
\hline $2786 / 167$ & $\begin{array}{l}\text { Aquila spilogaster } \\
\text { (Bonaparte, 1850) }\end{array}$ & African Hawk-Eagle & $\mathrm{m}$ & $\mathrm{R}(\mathrm{B})$ & $* *$ & & CITES \\
\hline
\end{tabular}




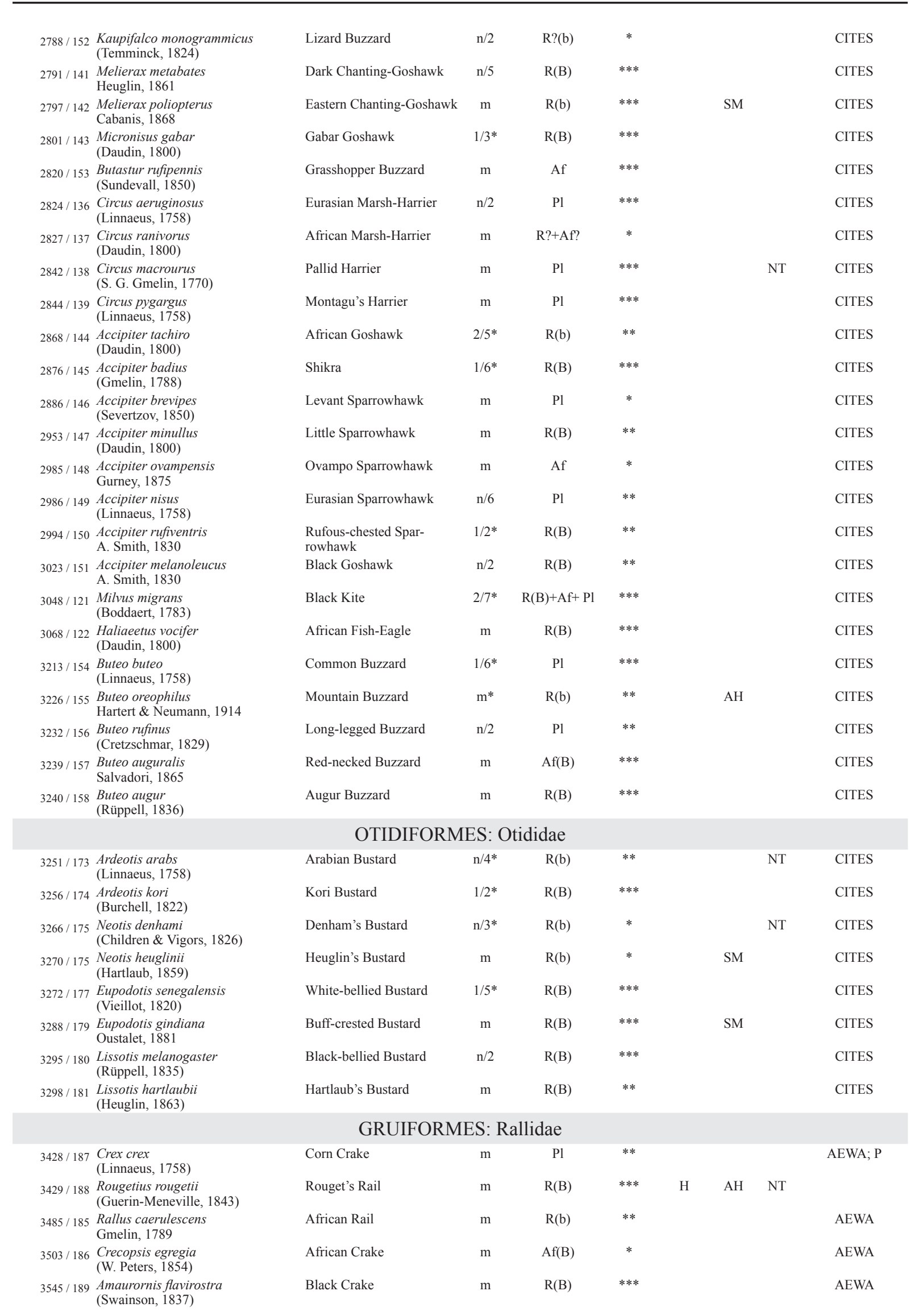




\begin{tabular}{|c|c|c|c|c|c|c|c|c|c|}
\hline $3556 / 191$ & $\begin{array}{l}\text { Porzana pusilla } \\
\text { (Pallas, 1776) }\end{array}$ & Baillon's Crake & $1 / 6^{*}$ & $\mathrm{R}(\mathrm{B})+\mathrm{Pl}$ ? & * & & & & AEWA \\
\hline $3565 / 192$ & $\begin{array}{l}\text { Porzana porzana } \\
\text { (Linnaeus, } 1766 \text { ) }\end{array}$ & Spotted Crake & $\mathrm{m}$ & Pl & $*$ & & & & AEWA \\
\hline $3628 / 194$ & $\begin{array}{l}\text { Porphyrio alleni } \\
\text { Thomson, } 1842\end{array}$ & Allen's Gallinule & $\mathrm{m}$ & $\operatorname{Af}(B)$ & $* *$ & & & & AEWA \\
\hline $3635 / 193$ & $\begin{array}{l}\text { Porphyrio madagascariensis } \\
\text { (Linneas, } 1758 \text { ) }\end{array}$ & Purple Swamphen & $\mathrm{m}^{*}$ & $\operatorname{Af}(B)$ & $* *$ & & & & \\
\hline $3655 / 195$ & $\begin{array}{l}\text { Gallinula chloropus } \\
\text { (Linnaeus, 1758) }\end{array}$ & Eurasian Moorhen & $1 / 5^{*}$ & $\mathrm{R}(\mathrm{B})$ & ** & & & & AEWA \\
\hline $3675 / 196$ & $\begin{array}{l}\text { Gallinula angulata } \\
\text { Sundevall, } 1850\end{array}$ & Lesser Moorhen & $\mathrm{m}$ & $\operatorname{Af}(B)$ & $* *$ & & & & AEWA \\
\hline $3679 / 197$ & $\begin{array}{l}\text { Fulica cristata } \\
\text { Gmelin, } 1789\end{array}$ & Red-knobbed Coot & $\mathrm{m}$ & $\mathrm{R}(\mathrm{B})$ & $* * *$ & & & & AEWA \\
\hline \multicolumn{10}{|c|}{ GRUIFORMES: Sarothruridae } \\
\hline $3714 / 182$ & $\begin{array}{l}\text { Sarothrura elegans } \\
\text { (A. Smith, 1839) }\end{array}$ & Buff-spotted Flufftail & $\mathrm{n} / 2$ & R?(b)+Af? & $*$ & & & \multirow{2}{*}{\multicolumn{2}{|c|}{ AEWA }} \\
\hline $3717 / 183$ & $\begin{array}{l}\text { Sarothrura rufa } \\
\text { (Vieillot, 1819) }\end{array}$ & Red-chested Flufftail & $1 / 3^{*}$ & $\mathrm{R} ?(\mathrm{~B})+\mathrm{Af} ?$ & $* *$ & & & & \\
\hline $3729 / 184$ & $\begin{array}{l}\text { Sarothrura ayresi } \\
\text { (Gurney, 1877) }\end{array}$ & White-winged Flufftail & $\mathrm{m}^{*}$ & $\mathrm{R} ?(\mathrm{~B})+\mathrm{Af} ?$ & $* *$ & & & EN & AEWA; P \\
\hline \multicolumn{10}{|c|}{ GRUIFORMES: Heliornithidae } \\
\hline $3732 / 198$ & $\begin{array}{l}\text { Podica senegalensis } \\
\text { (Vieillot, 1817) }\end{array}$ & African Finfoot & $\mathrm{n} / 4^{*}$ & $\mathrm{R}(\mathrm{b})$ & $* *$ & & & & \\
\hline \multicolumn{10}{|c|}{ GRUIFORMES: Gruidae } \\
\hline $3759 / 199$ & $\begin{array}{l}\text { Balearica pavonina } \\
\text { (Linnaeus, 1758) }\end{array}$ & Black Crowned-Crane & $1 / 2^{*}$ & $\mathrm{R}(\mathrm{B})+\mathrm{Af}$ & $* * *$ & & & VU & AEWA, CITES \\
\hline $3764 / 201$ & $\begin{array}{l}\text { Bugeranus carunculatus } \\
\text { (Gmelin, 1789) }\end{array}$ & Wattled Crane & $\mathrm{m}^{*}$ & $\mathrm{R}(\mathrm{B})$ & $* *$ & & & VU & AEWA, CITES; P \\
\hline $3780 / 202$ & $\begin{array}{l}\text { Grus grus } \\
\text { (Linnaeus, 1758) }\end{array}$ & Common Crane & $\mathrm{m}$ & $\mathrm{Pl}$ & $* * *$ & & & & AEWA, CITES \\
\hline \multicolumn{10}{|c|}{ CHARADRIIFORMES: Burhinidae } \\
\hline $3795 / 207$ & $\begin{array}{l}\text { Burhinus vermiculatus } \\
\text { (Cabanis, 1868) }\end{array}$ & Water Thick-knee & $\mathrm{n} / 2$ & $\begin{array}{c}\mathrm{R}(\mathrm{b}) \\
\mathrm{R}\end{array}$ & $*$ & & & & \\
\hline $3798 / 205$ & $\begin{array}{l}\text { Burhinus oedicnemus } \\
\text { (Linnaeus, 1758) }\end{array}$ & Eurasian Thick-knee & $\mathrm{n} / 5^{*}$ & $\mathrm{Pl}$ & $* *$ & & & & \\
\hline $3805 / 206$ & $\begin{array}{l}\text { Burhinus senegalensis } \\
\text { (Swainson, 1837) }\end{array}$ & Senegal Thick-knee & $\mathrm{m}$ & $\mathrm{R}(\mathrm{B})$ & $* * *$ & & & & AEWA \\
\hline $3806 / 208$ & $\begin{array}{l}\text { Burhinus capensis } \\
\text { (Lichtenstein, 1823) }\end{array}$ & Spotted Thick-knee & $1 / 4^{*}$ & $\mathrm{R}(\mathrm{B})$ & $* *$ & & & & \\
\hline \multicolumn{10}{|c|}{ CHARADRIIFORMES: Pluvianidae } \\
\hline $3821 / 265$ & $\begin{array}{l}\text { Pluvianus aegyptius } \\
\text { (Linnaeus, 1758) }\end{array}$ & Egyptian Plover & $\mathrm{m}$ & Af(B) & $* * *$ & & & & AEWA \\
\hline \multicolumn{10}{|c|}{ CHARADRIIFORMES: Recurvirostridae } \\
\hline $3822 / 211$ & $\begin{array}{l}\text { Himantopus himantopus } \\
\text { (Linnaeus, 1758) }\end{array}$ & Black-winged Stilt & $\mathrm{m}$ & $\mathrm{R}(\mathrm{B})+\mathrm{Pl}$ & $* * *$ & & & & AEWA; T \\
\hline $3834 / 212$ & $\begin{array}{l}\text { Recurvirostra avosetta } \\
\text { Linnaeus, } 1758\end{array}$ & Pied Avocet & $\mathrm{m}$ & Af & $* * *$ & & & & AEWA; T \\
\hline \multicolumn{10}{|c|}{ CHARADRIIFORMES: Charadriidae } \\
\hline $3862 / 223$ & $\begin{array}{l}\text { Pluvialis squatarola } \\
\text { (Linnaeus, 1758) }\end{array}$ & Black-bellied Plover & $\mathrm{m}$ & Pl & $* *$ & & & & AEWA \\
\hline $3867 / 222$ & $\begin{array}{l}\text { Pluvialis fulva } \\
\text { (Gmelin, 1789) }\end{array}$ & Pacific Golden-Plover & $\mathrm{m}$ & $\mathrm{Pl}$ & $* *$ & & & & AEWA \\
\hline $3875 / 213$ & $\begin{array}{l}\text { Vanellus crassirostris } \\
\text { (Hartlaub, 1855) }\end{array}$ & Long-toed Lapwing & $1 / 2^{*}$ & $\mathrm{R}(\mathrm{B})$ & $*$ & & & & \\
\hline $3879 / 214$ & $\begin{array}{l}\text { Vanellus spinosus } \\
\text { (Linnaeus, 1758) }\end{array}$ & Spur-winged Lapwing & $\mathrm{m}$ & $\mathrm{R}(\mathrm{B})$ & $* * *$ & & & & AEWA; T \\
\hline $3882 / 215$ & $\begin{array}{l}\text { Vanellus tectus } \\
\text { (Boddaert, 1783) }\end{array}$ & Black-headed Lapwing & $\mathrm{n} / 2^{*}$ & $\mathrm{R}(\mathrm{B})$ & $* * *$ & & & & \\
\hline $3887 / 216$ & $\begin{array}{l}\text { Vanellus melanopterus } \\
\text { (Cretzschmar, 1829) }\end{array}$ & Black-winged Lapwing & $\mathrm{n} / 2$ & $\mathrm{R}(\mathrm{B})$ & $* * *$ & & & & AEWA \\
\hline $3890 / 217$ & $\begin{array}{l}\text { Vanellus coronatus } \\
\text { (Boddaert, 1783) }\end{array}$ & Crowned Lapwing & $2 / 2^{*}$ & $\mathrm{R}(\mathrm{B})$ & $* * *$ & & & & AEWA \\
\hline $3893 / 218$ & $\begin{array}{l}\text { Vanellus senegallus } \\
\text { (Linnaeus, } 1766 \text { ) }\end{array}$ & Wattled Lapwing & $1 / 3^{*}$ & $\mathrm{R}(\mathrm{B})$ & $* * *$ & & & & AEWA \\
\hline $3897 / 219$ & $\begin{array}{l}\text { Vanellus melanocephalus } \\
\text { (Rüppell, 1845) }\end{array}$ & Spot-breasted Lapwing & $\mathrm{m}$ & $\mathrm{R}(\mathrm{B})$ & $* * *$ & $\mathrm{E}, \mathrm{H}$ & $\mathrm{AH}$ & & \\
\hline $3910 / 220$ & $\begin{array}{l}\text { Vanellus gregarius } \\
\text { (Pallas, 1771) }\end{array}$ & Sociable Lapwing & $\mathrm{m}$ & P1 & $*$ & & & $\mathrm{CR}$ & CMS, AEWA \\
\hline $3924 / 230$ & $\begin{array}{l}\text { Charadrius mongolus } \\
\text { Pallas, } 1776\end{array}$ & Lesser Sand-Plover & $1 / 5^{*}$ & $\mathrm{Pl}$ & $* *$ & & & & AEWA \\
\hline $3932 / 231$ & $\begin{array}{l}\text { Charadrius leschenaultii } \\
\text { Lesson, } 1826\end{array}$ & Greater Sand-Plover & $1 / 3^{*}$ & $\mathrm{Pl}$ & $*$ & & & & AEWA \\
\hline
\end{tabular}




\begin{tabular}{|c|c|c|c|c|c|c|c|}
\hline $3937 / 232$ & $\begin{array}{l}\text { Charadrius asiaticus } \\
\text { Pallas, } 1773\end{array}$ & Caspian Plover & $\mathrm{m}$ & $\mathrm{Pl}$ & $* * *$ & & AEWA \\
\hline $3944 / 226$ & $\begin{array}{l}\text { Charadrius pecuarius } \\
\text { Temminck, } 1823\end{array}$ & Kittlitz's Plover & $\mathrm{m}$ & $\mathrm{R}(\mathrm{B})$ & $* * *$ & & AEWA \\
\hline $3947 / 229$ & $\begin{array}{l}\text { Charadrius alexandrinus } \\
\text { Linnaeus, } 1758\end{array}$ & Kentish Plover & $\mathrm{n} / 3$ & Af?+Pl & $* * *$ & & AEWA \\
\hline $3961 / 224$ & $\begin{array}{l}\text { Charadrius hiaticula } \\
\text { Linnaeus, } 1758\end{array}$ & Common Ringed Plover & $1 / 2^{*}$ & $\mathrm{Pl}$ & $* * *$ & & AEWA \\
\hline $3969 / 225$ & $\begin{array}{l}\text { Charadrius dubius } \\
\text { Scopoli, } 1786\end{array}$ & Little Ringed Plover & $1 / 3^{*}$ & $\mathrm{Pl}$ & $* * *$ & & AEWA \\
\hline $3974 / 227$ & $\begin{array}{l}\text { Charadrius tricollaris } \\
\text { Vieillot, } 1818\end{array}$ & Three-banded Plover & $\mathrm{n} / 2$ & $\mathrm{R}(\mathrm{B})$ & $* * *$ & & AEWA \\
\hline \multicolumn{8}{|c|}{ CHARADRIIFORMES: Rostratulidae } \\
\hline $4018 / 233$ & $\begin{array}{l}\text { Rostratula benghalensis } \\
\text { (Linnaeus, 1758) }\end{array}$ & Greater Painted-snipe & $\mathrm{m}$ & $\mathrm{R}(\mathrm{B})$ & $* *$ & & \\
\hline \multicolumn{8}{|c|}{ CHARADRIIFORMES: Jacanidae } \\
\hline $4021 / 234$ & $\begin{array}{l}\text { Microparra capensis } \\
\text { (A. Smith, 1839) }\end{array}$ & Lesser Jacana & $\mathrm{m}$ & $\mathrm{R}(\mathrm{b})$ & $* *$ & & \\
\hline $4022 / 235$ & $\begin{array}{l}\text { Actophilornis africanus } \\
\text { (Gmelin, 1789) }\end{array}$ & African Jacana & $\mathrm{m}$ & $\mathrm{R}(\mathrm{B})$ & $* * *$ & & $\mathrm{~T}$ \\
\hline \multicolumn{8}{|c|}{ CHARADRIIFORMES: Scolopacidae } \\
\hline $4045 / 251$ & $\begin{array}{l}\text { Xenus cinereus } \\
\text { (Güldenstädt, 1775) }\end{array}$ & Terek Sandpiper & $\mathrm{m}$ & Pl & $* *$ & & AEWA \\
\hline $4046 / 252$ & $\begin{array}{l}\text { Actitis hypoleucos } \\
\text { (Linnaeus, 1758) }\end{array}$ & Common Sandpiper & $\mathrm{m}$ & Pl & $* * *$ & & AEWA \\
\hline $4049 / 249$ & $\begin{array}{l}\text { Tringa ochropus } \\
\text { Linnaeus, } 1758\end{array}$ & Green Sandpiper & $\mathrm{m}$ & Pl & $* * *$ & & AEWA \\
\hline $4056 / 245$ & $\begin{array}{l}\text { Tringa erythropus } \\
\text { (Pallas, 1764) }\end{array}$ & Spotted Redshank & $\mathrm{m}$ & Pl & $* * *$ & & AEWA \\
\hline $4058 / 248$ & $\begin{array}{l}\text { Tringa nebularia } \\
\text { (Gunnerus, 1767) }\end{array}$ & Common Greenshank & $\mathrm{m}$ & $\mathrm{Pl}$ & $* * *$ & & AEWA \\
\hline $4065 / 247$ & $\begin{array}{l}\text { Tringa stagnatilis } \\
\text { (Bechstein, 1803) }\end{array}$ & Marsh Sandpiper & $\mathrm{m}$ & $\mathrm{Pl}$ & $* * *$ & & AEWA \\
\hline $4066 / 250$ & $\begin{array}{l}\text { Tringa glareola } \\
\text { Linnaeus, } 1758\end{array}$ & Wood Sandpiper & $\mathrm{m}$ & $\mathrm{Pl}$ & $* * *$ & & AEWA \\
\hline $4067 / 246$ & $\begin{array}{l}\text { Tringa totanus } \\
\text { (Linnaeus, 1758) }\end{array}$ & Common Redshank & $2 / 6^{*}$ & Pl & $* * *$ & & AEWA \\
\hline $4078 / 242$ & $\begin{array}{l}\text { Numenius phaeopus } \\
\text { (Linnaeus, 1758) }\end{array}$ & Whimbrel & $\mathrm{n} / 4$ & $\mathrm{Pl}$ & $*$ & & AEWA \\
\hline $4087 / 244$ & $\begin{array}{l}\text { Numenius arquata } \\
\text { (Linnaeus, 1758) }\end{array}$ & Eurasian Curlew & $1 / 2^{*}$ & Pl & $* *$ & NT & AEWA \\
\hline $4095 / 240$ & $\begin{array}{l}\text { Limosa limosa } \\
\text { (Linnaeus, 1758) }\end{array}$ & Black-tailed Godwit & $\mathrm{n} / 3$ & $\mathrm{Pl}$ & $* *$ & NT & AEWA \\
\hline $4109 / 253$ & $\begin{array}{l}\text { Arenaria interpres } \\
\text { (Linnaeus, 1758) }\end{array}$ & Ruddy Turnstone & $\mathrm{n} / 2$ & $\mathrm{Pl}$ & $* * *$ & & AEWA \\
\hline $4128 / 262$ & $\begin{array}{l}\text { Calidris pugnax } \\
\text { (Linnaeus, 1758) }\end{array}$ & Ruff & $\mathrm{m}$ & $\mathrm{Pl}$ & $* * *$ & & AEWA \\
\hline $4129 / 261$ & $\begin{array}{l}\text { Calidris falcinellus } \\
\text { (Pontoppidan, 1763) }\end{array}$ & Broad-billed Sandpiper & $\mathrm{n} / 2$ & Pl & $*$ & & AEWA \\
\hline $4134 / 259$ & $\begin{array}{l}\text { Calidris ferruginea } \\
\text { (Pontoppidan, 1763) }\end{array}$ & Curlew Sandpiper & $\mathrm{m}$ & $\mathrm{Pl}$ & $* * *$ & & AEWA \\
\hline $4135 / 256$ & $\begin{array}{l}\text { Calidris temminckii } \\
\text { (Leisler, 1812) }\end{array}$ & Temminck's Stint & $\mathrm{m}$ & $\mathrm{Pl}$ & $* * *$ & & AEWA \\
\hline $4136 / 257$ & $\begin{array}{l}\text { Calidris subminuta } \\
\text { (Middendorff, 1853) }\end{array}$ & Long-toed Stint & $\mathrm{m}$ & Pl & $*$ & & \\
\hline $4139 / 254$ & $\begin{array}{l}\text { Calidris alba } \\
\text { (Pallas, 1764) }\end{array}$ & Sanderling & $\mathrm{m}$ & $\mathrm{Pl}$ & $*$ & & AEWA \\
\hline $4140 / 260$ & $\begin{array}{l}\text { Calidris alpina } \\
\text { (Linnaeus, 1758) }\end{array}$ & Dunlin & $\mathrm{n} / 9^{*}$ & $\mathrm{Pl}$ & $* *$ & & AEWA \\
\hline $4161 / 255$ & $\begin{array}{l}\text { Calidris minuta } \\
\text { (Leisler, 1812) }\end{array}$ & Little Stint & $\mathrm{m}$ & $\mathrm{Pl}$ & $* * *$ & & AEWA \\
\hline $4168 / 258$ & $\begin{array}{l}\text { Calidris melanotos } \\
\text { (Vieillot, 1819) }\end{array}$ & Pectoral Sandpiper & $\mathrm{m}$ & Pl & $*$ & & \\
\hline $4192 / 236$ & $\begin{array}{l}\text { Lymnocryptes minimus } \\
\text { (Brunnich, 1764) }\end{array}$ & Jack Snipe & $\mathrm{m}$ & $\mathrm{Pl}$ & $* *$ & & AEWA \\
\hline $4198 / 238$ & $\begin{array}{l}\text { Gallinago media } \\
\text { (Latham, 1787) }\end{array}$ & Great Snipe & $\mathrm{m}$ & Pl & $* * *$ & NT & AEWA; H \\
\hline $4200 / 239$ & $\begin{array}{l}\text { Gallinago gallinago } \\
\text { (Linnaeus, 1758) }\end{array}$ & Common Snipe & $\mathrm{n} / 2$ & $\mathrm{Pl}$ & $* * *$ & & AEWA; H \\
\hline $4220 / 237$ & $\begin{array}{l}\text { Gallinago nigripennis } \\
\text { Bonaparte, } 1839\end{array}$ & African Snipe & $1 / 3^{*}$ & $\mathrm{R}(\mathrm{B})$ & $* * *$ & & \\
\hline $4235 / 263$ & $\begin{array}{l}\text { Phalaropus lobatus } \\
\text { (Linnaeus, 1758) }\end{array}$ & Red-necked Phalarope & $\mathrm{m}$ & Pl & $* *$ & & AEWA \\
\hline $4236 / 264$ & $\begin{array}{l}\text { Phalaropus fulicarius } \\
\text { (Linnaeus, 1758) }\end{array}$ & Red Phalarope & $\mathrm{m}$ & $\mathrm{Pl}$ & $*$ & & AEWA \\
\hline
\end{tabular}




\begin{tabular}{|c|c|c|c|c|c|c|c|c|c|}
\hline \multicolumn{10}{|c|}{ CHARADRIIFORMES: Turnicidae } \\
\hline $4241 / 203$ & $\begin{array}{l}\text { Turnix sylvaticus } \\
\text { (Desfontaines, 1789) }\end{array}$ & Small Buttonquail & $1 / 9 *$ & $\mathrm{R} ?(\mathrm{~B})+\mathrm{Af} ?$ & $* *$ & & & & \\
\hline $4307 / 204$ & $\begin{array}{l}\text { Ortyxelos meiffrenii } \\
\text { (Vieillot, 1819) }\end{array}$ & Quail-plover & $\mathrm{m}$ & R?+Af? & $*$ & & & & \\
\hline \multicolumn{10}{|c|}{ CHARADRIIFORMES: Glareolidae } \\
\hline $4313 / 267$ & $\begin{array}{l}\text { Cursorius somalensis } \\
\text { Shelley, } 1885\end{array}$ & Somali Courser & $2 / 2 *$ & $\mathrm{R}(\mathrm{B})$ & $* *$ & $\mathrm{H}$ & SM & & \\
\hline $4317 / 268$ & $\begin{array}{l}\text { Cursorius temminckii } \\
\text { Swainson, } 1822\end{array}$ & Temminck's Courser & $\mathrm{m}$ & $\mathrm{R}(\mathrm{B})$ & $* * *$ & & & & \\
\hline $4319 / 269$ & $\begin{array}{l}\text { Smutsornis africanus } \\
\text { (Temminck, 1807) }\end{array}$ & Double-banded Courser & $2 / 8^{*}$ & $\mathrm{R}(\mathrm{B})$ & $* *$ & & & & \\
\hline $4328 / 270$ & $\begin{array}{l}\text { Rhinoptilus cinctus } \\
\text { (Heuglin, 1863) }\end{array}$ & Three-banded Courser & $\mathrm{n} / 3^{*}$ & $\mathrm{R}(\mathrm{B})$ & $* * *$ & & & & \\
\hline $4332 / 271$ & $\begin{array}{l}\text { Rhinoptilus chalcopterus } \\
\text { (Temminck, 1824) }\end{array}$ & Bronze-winged Courser & $\mathrm{m}$ & Af & $*$ & & & & \\
\hline $4336 / 272$ & $\begin{array}{l}\text { Glareola pratincola } \\
\text { (Linnaeus, 1766) }\end{array}$ & Collared Pratincole & $\mathrm{n} / 3^{*}$ & $\mathrm{R}(\mathrm{B})+\mathrm{Pl}$ & $* *$ & & & & AEWA \\
\hline $4342 / 273$ & $\begin{array}{l}\text { Glareola nordmanni } \\
\text { Fischer von Waldheim, } 1842\end{array}$ & Black-winged Pratincole & $\mathrm{m}$ & $\mathrm{Pl}$ & $*$ & & & NT & AEWA \\
\hline $4345 / 275$ & $\begin{array}{l}\text { Glareola nuchalis } \\
\text { G. R. Gray, } 1849\end{array}$ & Rock Pratincole & $\mathrm{n} / 2$ & $\mathrm{R}(\mathrm{b})$ & $*$ & & & & AEWA \\
\hline \multicolumn{10}{|c|}{ CHARADRIIFORMES: Stercorariidae } \\
\hline $4361 / 301$ & $\begin{array}{l}\text { Stercorarius parasiticus } \\
\text { (Linnaeus, 1758) }\end{array}$ & Parasitic Jaeger & $\mathrm{m}$ & Pl & $*$ & & & & \\
\hline \multicolumn{10}{|c|}{ CHARADRIIFORMES: Laridae } \\
\hline $4450 / 283$ & $\begin{array}{l}\text { Chroicocephalus genei } \\
\text { (Breme, 1839) }\end{array}$ & Slender-billed Gull & $\mathrm{m}$ & $\mathrm{Pl}$ & $*$ & & & & AEWA \\
\hline $4461 / 281$ & $\begin{array}{l}\text { Chroicocephalus cirrocephalus } \\
\text { (Vieillot, 1818) }\end{array}$ & Gray-hooded Gull & $1 / 2^{*}$ & Af(B) & $* * *$ & & & & AEWA \\
\hline $4465 / 282$ & $\begin{array}{l}\text { Chroicocephalus ridibundus } \\
\text { (Linnaeus, 1766) }\end{array}$ & Black-headed Gull & $\mathrm{m}$ & $\mathrm{Pl}$ & $* * *$ & & & & AEWA \\
\hline $4486 / 280$ & $\begin{array}{l}\text { Ichthyaetus ichthyaetus (Pallas, } \\
1773 \text { ) }\end{array}$ & Pallas's Gull & $\mathrm{m}$ & $\mathrm{Pl}$ & $* *$ & & & & AEWA \\
\hline $4537 / 278$ & $\begin{array}{l}\text { Larus fuscus } \\
\text { Linnaeus, } 1758\end{array}$ & Lesser Black-backed Gull & $2 / 5^{*}$ & $\mathrm{Pl}$ & $* * *$ & & & & AEWA \\
\hline $4611 / 294$ & $\begin{array}{l}\text { Onychoprion fuscatus } \\
\text { (Linnaeus, 1766) }\end{array}$ & Sooty Tern & $1 / 8^{*}$ & $\mathrm{Pl}$ & $*$ & & & & AEWA \\
\hline $4651 / 292$ & $\begin{array}{l}\text { Sternula saundersi } \\
\text { (Hume, 1877) }\end{array}$ & Saunders's Tern & $\mathrm{m}$ & Af? & $*$ & & & & AEWA \\
\hline $4657 / 284$ & $\begin{array}{l}\text { Gelochelidon nilotica (Gmelin, } \\
1789)\end{array}$ & Gull-billed Tern & $\mathrm{n} / 6$ & $\mathrm{Pl}$ & $* * *$ & & & & AEWA \\
\hline $4665 / 285$ & $\begin{array}{l}\text { Hydroprogne caspia } \\
\text { (Pallas, 1770) }\end{array}$ & Caspian Tern & $\mathrm{m}$ & $\mathrm{Af}+\mathrm{Pl}$ & $* *$ & & & & AEWA \\
\hline $4667 / 297$ & $\begin{array}{l}\text { Chlidonias niger } \\
\text { (Linnaeus, 1758) }\end{array}$ & Black Tern & $\mathrm{n} / 2$ & Pl & $*$ & & & & AEWA \\
\hline $4670 / 296$ & $\begin{array}{l}\text { Chlidonias leucopterus } \\
\text { (Temminck, 1815) }\end{array}$ & White-winged Tern & $\mathrm{m}$ & $\mathrm{Pl}$ & $* * *$ & & & & AEWA \\
\hline $4671 / 295$ & $\begin{array}{l}\text { Chlidonias hybrida } \\
\text { (Pallas, 1811) }\end{array}$ & Whiskered Tern & $\mathrm{n} / 7^{*}$ & Pl & $* * *$ & & & & AEWA \\
\hline $4694 / 289$ & $\begin{array}{l}\text { Sterna hirundo } \\
\text { Linnaeus, } 1758\end{array}$ & Common Tern & $\mathrm{n} / 4$ & Pl & $*$ & & & & AEWA \\
\hline $4729 / 287$ & $\begin{array}{l}\text { Thalasseus sandvicensis } \\
\text { (Latham, 1787) }\end{array}$ & Sandwich Tern & $\mathrm{n} / 3$ & $\mathrm{Pl}$ & $*$ & & & & AEWA \\
\hline $4748 / 299$ & $\begin{array}{l}\text { Rynchops flavirostris } \\
\text { Vieillot, } 1816\end{array}$ & African Skimmer & $\mathrm{m}$ & Af & $* *$ & & & NT & AEWA \\
\hline \multicolumn{10}{|c|}{ PTEROCLIFORMES: Pteroclidae } \\
\hline $4757 / 302$ & $\begin{array}{l}\text { Pterocles exustus } \\
\text { Temminck, } 1825\end{array}$ & $\begin{array}{l}\text { Chestnut-bellied Sand- } \\
\text { grouse }\end{array}$ & $2 / 6^{*}$ & $\mathrm{R}(\mathrm{B})$ & $* * *$ & & & & $\mathrm{~T}, \mathrm{H}$ \\
\hline 47697304 & $\begin{array}{l}\text { Pterocles gutturalis } \\
\text { A. Smith, } 1836\end{array}$ & $\begin{array}{l}\text { Yellow-throated Sand- } \\
\text { grouse }\end{array}$ & $1 / 2 *$ & $\mathrm{R} ?(\mathrm{~b})+\mathrm{Af} ?$ & $* * *$ & & & & $\mathrm{~T}, \mathrm{H}$ \\
\hline $4778 / 305$ & $\begin{array}{l}\text { Pterocles decoratus } \\
\text { Cabanis, } 1868\end{array}$ & Black-faced Sandgrouse & $1 / 3 *$ & $\mathrm{R} ?(\mathrm{~b})+\mathrm{Af} ?$ & $* * *$ & & SM & & $\mathrm{T}, \mathrm{H}$ \\
\hline $4783 / 306$ & $\begin{array}{l}\text { Pterocles lichtensteinii } \\
\text { Temminck, } 1825\end{array}$ & Lichtenstein's Sandgrouse & $2 / 5^{*}$ & $\mathrm{R} ?(\mathrm{~B})+\mathrm{Af} ?$ & $* * *$ & & & & $\mathrm{~T}, \mathrm{H}$ \\
\hline $4794 / 307$ & $\begin{array}{l}\text { Pterocles quadricinctus } \\
\text { Temminck, } 1815\end{array}$ & Four-banded Sandgrouse & $\mathrm{m}$ & $\mathrm{R}(\mathrm{B})$ & $* * *$ & & & & $\mathrm{~T}, \mathrm{H}$ \\
\hline \multicolumn{10}{|c|}{ COLUMBIFORMES: Columbidae } \\
\hline $4798 / 308$ & $\begin{array}{l}\text { Columba livia } \\
\text { Gmelin, } 1789\end{array}$ & $\begin{array}{l}\text { Rock Pigeon (Feral Pi- } \\
\text { geon) }\end{array}$ & $-/ 13^{*}$ & $\mathrm{R}(\mathrm{B})$ & $* * *$ & & & & $\mathrm{~T}$ \\
\hline $4820 / 309$ & $\begin{array}{l}\text { Columba guinea } \\
\text { Linnaeus, } 1758\end{array}$ & Speckled Pigeon & $\mathrm{n} / 2$ & $\mathrm{R}(\mathrm{B})$ & $* * *$ & & & & $\mathrm{~T}, \mathrm{H}$ \\
\hline
\end{tabular}




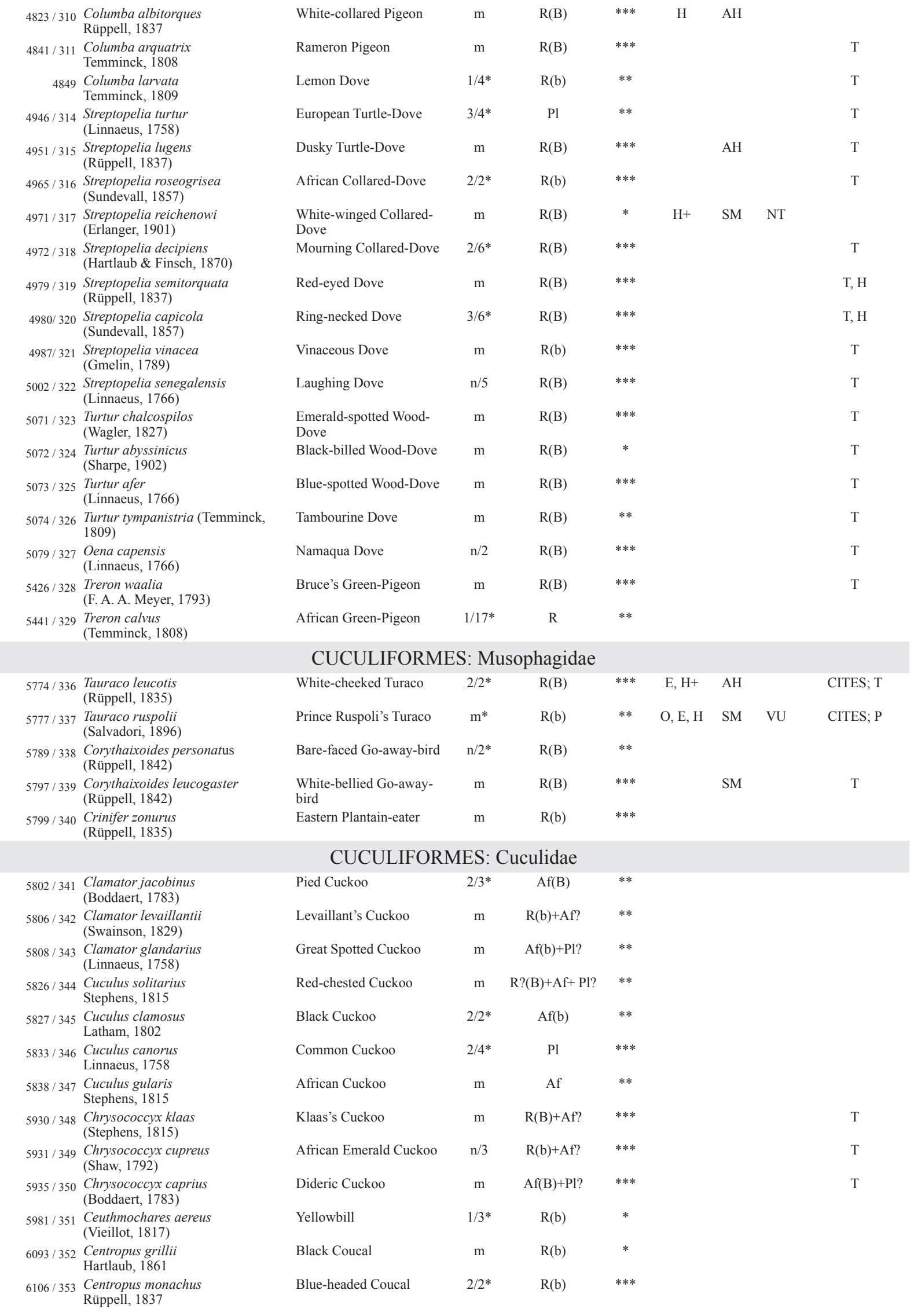




\begin{tabular}{|c|c|c|c|c|}
\hline $6110 / 354$ & $\begin{array}{l}\text { Centropus senegalensis } \\
\text { (Linnaeus, 1766) }\end{array}$ & Senegal Coucal & $\mathrm{n} / 3$ & $\mathrm{R}(\mathrm{b})$ \\
\hline \multirow[t]{2}{*}{$6114 / 355$} & $\begin{array}{l}\text { Centropus superciliosus } \\
\text { Hemprich \& Ehrenberg, } 1829\end{array}$ & White-browed Coucal & $\mathrm{n} / 4^{*}$ & $\mathrm{R}(\mathrm{B})$ \\
\hline & & \multicolumn{3}{|c|}{ STRIGIFORMES: Tytonidae } \\
\hline $6227 / 357$ & $\begin{array}{l}\text { Tyto capensis } \\
\text { (A. Smith, 1834) }\end{array}$ & African Grass-Owl & $\mathrm{m}$ & $\mathrm{R} ?+\mathrm{Af} ?$ \\
\hline \multirow[t]{2}{*}{$6228 / 356$} & $\begin{array}{l}\text { Tyto alba } \\
\text { (Scopoli, 1769) }\end{array}$ & Barn Owl & $1 / 29^{*}$ & $\mathrm{R}(\mathrm{B})$ \\
\hline & & \multicolumn{3}{|c|}{ STRIGIFORMES: Strigidae } \\
\hline $6362 / 359$ & $\begin{array}{l}\text { Otus scops } \\
\text { (Linnaeus, 1758) }\end{array}$ & European Scops-Owl & $2 / 6^{*}$ & $\mathrm{Pl}$ \\
\hline $6372 / 358$ & $\begin{array}{l}\text { Otus senegalensis } \\
\text { (Swainson, 1837) }\end{array}$ & African Scops-Owl & $\mathrm{n} / 3$ & $\mathrm{R}(\mathrm{B})$ \\
\hline $6491 / 360$ & $\begin{array}{l}\text { Ptilopsis leucotis } \\
\text { (Temminck, 1820) }\end{array}$ & Northern White-faced Owl & $\mathrm{m}$ & $\mathrm{R}(\mathrm{B})$ \\
\hline $6544 / 362$ & $\begin{array}{l}\text { Bubo capensis } \\
\text { A. Smith, } 1834\end{array}$ & Cape Eagle-Owl & $1 / 3^{*}$ & $\mathrm{R}(\mathrm{B})$ \\
\hline $6554 / 363$ & $\begin{array}{l}\text { Bubo cinerascens } \\
\text { Guerin-Meneville, } 1843\end{array}$ & Grayish Eagle-Owl & $\mathrm{m}$ & $\mathrm{R}(\mathrm{B})$ \\
\hline $6564 / 364$ & $\begin{array}{l}\text { Bubo lacteus } \\
\text { (Temminck, 1820) }\end{array}$ & Verreaux's Eagle-Owl & $\mathrm{m}$ & $\mathrm{R}(\mathrm{B})$ \\
\hline $6591 / 365$ & $\begin{array}{l}\text { Scotopelia peli } \\
\text { (Bonaparte, 1850) }\end{array}$ & Pel's Fishing-Owl & $\mathrm{m}$ & $\mathrm{R}(\mathrm{b})$ \\
\hline $6607 / 367$ & $\begin{array}{l}\text { Glaucidium perlatum } \\
\text { (Vieillot, 1817) }\end{array}$ & Pearl-spotted Owlet & $1 / 2^{*}$ & $\mathrm{R}(\mathrm{B})$ \\
\hline $6823 / 366$ & $\begin{array}{l}\text { Strix woodfordii } \\
\text { (A. Smith, 1834) }\end{array}$ & African Wood-Owl & $1 / 4^{*}$ & $\mathrm{R}(\mathrm{B})$ \\
\hline $6835 / 369$ & $\begin{array}{l}\text { Asio abyssinicus } \\
\text { (Guerin-Meneville, 1843) }\end{array}$ & African Long-eared Owl & $\mathrm{n} / 2^{*}$ & $\mathrm{R}(\mathrm{B})$ \\
\hline $6846 / 370$ & $\begin{array}{l}\text { Asio flammeus } \\
\text { (Pontoppidan, 1763) }\end{array}$ & Short-eared Owl & $\mathrm{n} / 10$ & $\mathrm{Pl}$ \\
\hline $6860 / 371$ & $\begin{array}{l}\text { Asio capensis } \\
\text { (A. Smith, 1834) }\end{array}$ & Marsh Owl & $\mathrm{n} / 3$ & $\mathrm{R} ?(\mathrm{~b})+\mathrm{Af}$ ? \\
\hline
\end{tabular}

7214 / 385 Caprimulgus longipennis

$7227 / 372$ Caprimulgus europaeus

Linnaeus, 1758

$7235 / 373$ Caprimulgus fraenatus Salvadori, 1884

7239/374 Caprimulgus aegyptius Lichtenstein, 1823

7242 / 375 Caprimulgus nubicus Lichtenstein, 1823

$7269 / 376$ Caprimulgus donaldsoni Sharpe, 1895

7276/377 Caprimulgus poliocephalus Rüppell, 1840

7287/378 Caprimulgus natalensis A. Smith, 1845

$7290 / 380$ Caprimulgus inornatus Heuglin, 1869

$7291 / 381$ Caprimulgus stellatus Blundell \& Lovat, 1899

$7304 / 382$ Caprimulgus tristigma Rüppell, 1840

7316/3383 Caprimulgus climacurus Vieillot, 1824

$7320 / 384$ Caprimulgus clarus Reichenow, 1892

CAPRIMULGIFORMES: Caprimulgidae

Standard-winged Nightjar $m \quad$ Af(B)
Eurasian Nightjar $\quad 2 / 6^{*}$

Sombre Nightjar

Egyptian Nightjar

Nubian Nightjar

Donaldson-Smith's

Nightjar

Abyssinian Nightjar

Swamp Nightjar

Plain Nightjar

Star-spotted Nightjar

Freckled Nightjar

Long-tailed Nightjar

Slender-tailed Nightjar $\mathrm{m}$

$\mathrm{R}(\mathrm{B})+\mathrm{Af}$ ?

$\mathrm{n} / 2$

$1 / 4^{*}$

$\mathrm{m}$

$\mathrm{m}$

m

$\mathrm{n} / 2$

$\mathrm{m}$

$\mathrm{m}$

$\mathrm{m}$

$\mathrm{n} / 5$

$1 / 3^{*}$

$\mathrm{m}$
$\mathrm{AH}$

SM

$\mathrm{AH}$

SM

\section{APODIFORMES: Apodidae}

Af

$1 / 10 * \quad \mathrm{R}(\mathrm{b})+\mathrm{Af?}+$

$\begin{array}{ccc}1 / 10 * & \mathrm{R}(\mathrm{b})+\mathrm{Af} ?+ & * * * \\ & \mathrm{Pl} ? \\ \mathrm{n} / 4 & \mathrm{R}(\mathrm{b}) & * * * \\ 1 / 2 * & \mathrm{Pl} & * * * \\ 1 / 2 * & \mathrm{R}(\mathrm{B}) & * * * \\ 1 / 7 * & \mathrm{Af} ? & *\end{array}$

$\mathrm{AH}$

$\mathrm{AH}$
Scarce Swift

Alpine Swift

Mottled Swift

Common Swift

Nyanza Swift

African Swift

$\mathrm{n} / 3$

Apus melba
(Linnaeus, 1758

7596/389 Apus aequatorialis (J. W. von Müller, 1851)

7602/390 Apus apus (Linnaeus, 1758)

7606/391 Apus niansae (Reichenow, 1887)

7615 / - Apus barbatus (P.L. Sclater, 1866)

African Swift 


\begin{tabular}{|c|c|c|c|c|c|c|c|}
\hline $7641 / 392$ & $\begin{array}{l}\text { Apus affinis } \\
\text { (J. E. Gray, 1830) }\end{array}$ & Little Swift & $1 / 6^{*}$ & $\mathrm{R}(\mathrm{B})$ & $* * *$ & & \\
\hline $7654 / 393$ & $\begin{array}{l}\text { Apus horus } \\
\text { (Heuglin, 1869) }\end{array}$ & Horus Swift & $\mathrm{n} / 2$ & $\mathrm{R}(\mathrm{B})$ & $* * *$ & & \\
\hline $7657 / 394$ & $\begin{array}{l}\text { Apus caffer } \\
\text { (Lichtenstein, 1823) }\end{array}$ & White-rumped Swift & $\mathrm{m}$ & $\operatorname{Af}(B)$ & $* * *$ & & \\
\hline $7680 / 387$ & $\begin{array}{l}\text { Cypsiurus parvus (Lichtenstein, } \\
\text { 1823) }\end{array}$ & African Palm-Swift & $1 / 3^{*}$ & $\mathrm{R}(\mathrm{B})$ & $* *$ & & \\
\hline \multicolumn{8}{|c|}{ COLIIFORMES: Coliidae } \\
\hline $8647 / 395$ & $\begin{array}{l}\text { Colius striatus } \\
\text { Gmelin, } 1789\end{array}$ & Speckled Mousebird & $1 / 17^{*}$ & $\mathrm{R}(\mathrm{B})$ & $* * *$ & & \\
\hline $8670 / 396$ & $\begin{array}{l}\text { Urocolius macrourus } \\
\text { (Linnaeus, 1766) }\end{array}$ & Blue-naped Mousebird & $2 / 6^{*}$ & $\mathrm{R}(\mathrm{B})$ & $* * *$ & & $\mathrm{~T}$ \\
\hline \multicolumn{8}{|c|}{ TROGONIFORMES: Trogonidae } \\
\hline $8795 / 397$ & $\begin{array}{l}\text { Apaloderma narina } \\
\text { (Stephens, 1815) }\end{array}$ & Narina Trogon & $\mathrm{n} / 4$ & $\mathrm{R}(\mathrm{B})$ & $* *$ & & \\
\hline \multicolumn{8}{|c|}{ BUCEROTIFORMES: Upupidae } \\
\hline $8847 / 424$ & $\begin{array}{l}\text { Upupa epops } \\
\text { Linnaeus, } 1758\end{array}$ & Eurasian Hoopoe & $3 / 9^{*}$ & $\mathrm{R} ?(\mathrm{~B})+\mathrm{Af}+\mathrm{Pl}$ & $* * *$ & & $\mathrm{~T}$ \\
\hline \multicolumn{8}{|c|}{ BUCEROTIFORMES: Phoeniculidae } \\
\hline $8860 / 425$ & $\begin{array}{l}\text { Phoeniculus purpureus } \\
\text { (J. F. Miller, 1784) }\end{array}$ & Green Woodhoopoe & $1 / 6^{*}$ & $\mathrm{R}(\mathrm{b})$ & $*$ & & \\
\hline $8871 / 426$ & $\begin{array}{l}\text { Phoeniculus somaliensis } \\
\text { (Ogilvie-Grant, 1901) }\end{array}$ & Black-billed Woodhoopoe & $2 / 3^{*}$ & $\mathrm{R}(\mathrm{B})$ & $* * *$ & SM & \\
\hline $8883 / 427$ & $\begin{array}{l}\text { Rhinopomastus aterrimus } \\
\text { (Stephens, 1826) }\end{array}$ & Black Scimitar-bill & $1 / 4^{*}$ & $\mathrm{R}(\mathrm{B})$ & $* *$ & & \\
\hline $8891 / 428$ & $\begin{array}{l}\text { Rhinopomastus minor } \\
\text { (Rüppell, 1845) }\end{array}$ & Abyssinian Scimitar-bill & $2 / 2^{*}$ & $\mathrm{R}(\mathrm{B})$ & $* *$ & SM & \\
\hline \multicolumn{8}{|c|}{ BUCEROTIFORMES: Bucorvidae } \\
\hline $8894 / 437$ & $\begin{array}{l}\text { Bucorvus abyssinicus } \\
\text { (Boddaert, 1783) }\end{array}$ & $\begin{array}{l}\text { Abyssinian Ground- } \\
\text { Hornbill }\end{array}$ & $\mathrm{m}$ & $\mathrm{R}(\mathrm{B})$ & $* * *$ & & \\
\hline \multicolumn{8}{|c|}{ BUCEROTIFORMES: Bucerotidae } \\
\hline $8897 / 429$ & $\begin{array}{l}\text { Lophoceros alboterminatus } \\
\text { Büttikofer, } 1889\end{array}$ & Crowned Hornbill & $\mathrm{m}$ & $\mathrm{R}(\mathrm{B})$ & $* *$ & & \\
\hline $8902 / 430$ & $\begin{array}{l}\text { Lophoceros hemprichii } \\
\text { (Ehrenberg, 1833) }\end{array}$ & Hemprich's Hornbill & $\mathrm{m}$ & $\mathrm{R}(\mathrm{B})$ & $* * *$ & SM & \\
\hline 8903 & $\begin{array}{l}\text { Lophoceros nasutus } \\
\text { (Linnaeus, 1766) }\end{array}$ & African Gray Hornbill & $\mathrm{n} / 2$ & $\mathrm{R}(\mathrm{B})$ & $* * *$ & & \\
\hline $8910 / 433$ & $\begin{array}{l}\text { Tockus flavirostris } \\
\text { (Rüppell, 1835) }\end{array}$ & $\begin{array}{l}\text { Eastern Yellow-billed } \\
\text { Hornbill }\end{array}$ & $\mathrm{m}$ & $\mathrm{R}(\mathrm{B})$ & $* *$ & SM & \\
\hline $8912 / 435$ & $\begin{array}{l}\text { Tockus jacksoni } \\
\text { (Ogilvie-Grant, 1891) }\end{array}$ & Jackson’s Hornbill & $\mathrm{m}^{*}$ & $\mathrm{R}(\mathrm{b})$ & * & SM & \\
\hline $8913 / 434$ & $\begin{array}{l}\text { Tockus deckeni } \\
\text { (Cabanis, 1868) }\end{array}$ & Von der Decken's Hornbill & $\mathrm{m}^{*}$ & $\mathrm{R}(\mathrm{B})$ & $* * *$ & SM & $\mathrm{T}$ \\
\hline $8920 / 432$ & $\begin{array}{l}\text { Tockus erythrorhynchus } \\
\text { (Temminck, 1823) }\end{array}$ & $\begin{array}{l}\text { Northern Red-billed } \\
\text { Hornbill }\end{array}$ & $\mathrm{m}$ & $\mathrm{R}(\mathrm{B})$ & $* * *$ & & $\mathrm{~T}$ \\
\hline $8932 / 436$ & $\begin{array}{l}\text { Ceratogymna brevis } \\
\text { (Friedmann, 1929) }\end{array}$ & Silvery-cheeked Hornbill & $\mathrm{m}$ & $\mathrm{R}(\mathrm{B})$ & $* * *$ & & $\mathrm{~T}$ \\
\hline \multicolumn{8}{|c|}{ CORACIIFORMES: Alcedinidae } \\
\hline $9079 / 410$ & $\begin{array}{l}\text { Alcedo semitorquata } \\
\text { Swainson, } 1823\end{array}$ & Half-collared Kingfisher & $1 / 3^{*}$ & $\mathrm{R}(\mathrm{b})$ & ** & & \\
\hline $9157 / 409$ & $\begin{array}{l}\text { Corythornis cristatus } \\
\text { (Pallas 1764) }\end{array}$ & Malachite Kingfisher & $1 / 5^{*}$ & $\mathrm{R}(\mathrm{B})$ & $* *$ & & $\mathrm{~T}$ \\
\hline $9174 / 408$ & $\begin{array}{l}\text { Ispidina picta } \\
\text { (Boddaert, 1783) }\end{array}$ & African Pygmy-Kingfisher & $n / 3$ & $\mathrm{R}(\mathrm{B})$ & $* *$ & & \\
\hline $9244 / 403$ & $\begin{array}{l}\text { Halcyon leucocephala } \\
\text { (Statius Müller, 1776) }\end{array}$ & Gray-headed Kingfisher & $\mathrm{n} / 5^{*}$ & $\mathrm{R}(\mathrm{B})+\mathrm{Af}$ & $* * *$ & & $\mathrm{~T}$ \\
\hline $9252 / 406$ & $\begin{array}{l}\text { Halcyon senegalensis } \\
\text { (Linnaeus, 1766) }\end{array}$ & Woodland Kingfisher & $\mathrm{n} / 3^{*}$ & $\mathrm{R}(\mathrm{B})$ & $* * *$ & & $\mathrm{~T}$ \\
\hline $9259 / 405$ & $\begin{array}{l}\text { Halcyon malimbica } \\
\text { (Shaw, 1811) }\end{array}$ & Blue-breasted Kingfisher & $\mathrm{n} / 4^{*}$ & $\mathrm{R}(\mathrm{b})$ & $*$ & & \\
\hline $9269 / 404$ & $\begin{array}{l}\text { Halcyon chelicuti } \\
\text { (Stanley, 1814) }\end{array}$ & Striped Kingfisher & $\mathrm{n} / 2$ & $\mathrm{R}(\mathrm{B})$ & $* * *$ & & \\
\hline $9447 / 411$ & $\begin{array}{l}\text { Megaceryle maxima } \\
\text { (Pallas, 1769) }\end{array}$ & Giant Kingfisher & $\mathrm{n} / 2$ & $\mathrm{R}(\mathrm{B})$ & $* *$ & & $\mathrm{~T}$ \\
\hline $9461 / 412$ & $\begin{array}{l}\text { Ceryle rudis } \\
\text { (Linnaeus, 1758) }\end{array}$ & Pied Kingfisher & $\mathrm{n} / 4$ & $\mathrm{R}(\mathrm{B})+\mathrm{Af}$ & $* * *$ & & $\mathrm{~T}$ \\
\hline \multicolumn{8}{|c|}{ CORACIIFORMES: Meropidae } \\
\hline $9489 / 416$ & $\begin{array}{l}\text { Merops bulocki } \\
\text { Vieillot, } 1817\end{array}$ & Red-throated Bee-eater & $1 / 2^{*}$ & $\mathrm{R}$ ?(b)+Af? & * & SG & \\
\hline
\end{tabular}




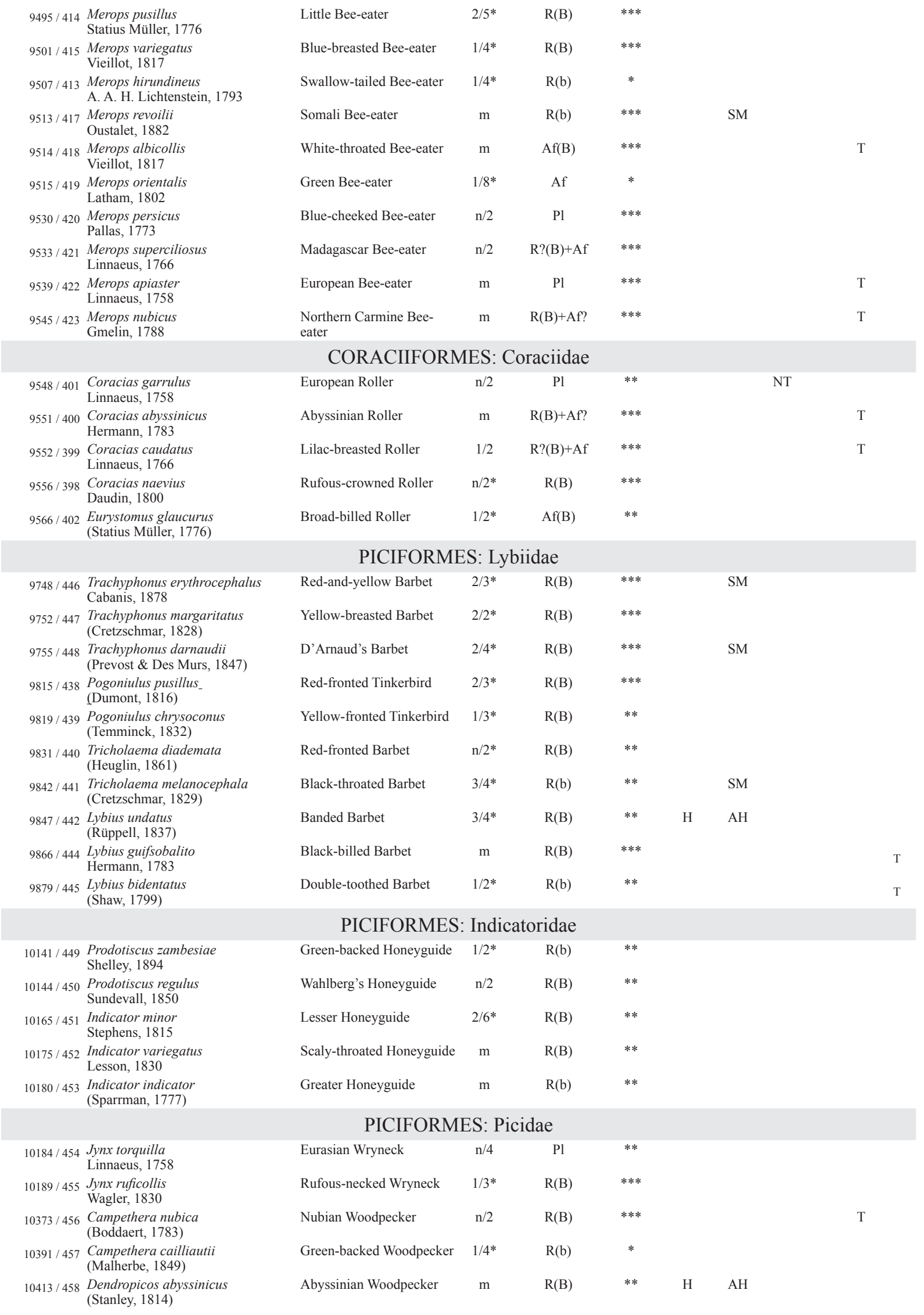




\begin{tabular}{|c|c|c|c|c|c|c|c|c|c|}
\hline $10414 / 459$ & $\begin{array}{l}\text { Dendropicos fuscescens } \\
\text { (Vieillot, 1818) }\end{array}$ & Cardinal Woodpecker & $3 / 9^{*}$ & $\mathrm{R}(\mathrm{B})$ & $* * *$ & & & & \\
\hline $10429 / 460$ & $\begin{array}{l}\text { Dendropicos namaquus } \\
\text { (A. A. H. Lichtenstein, 1793) }\end{array}$ & Bearded Woodpecker & $1 / 3^{*}$ & $\mathrm{R}(\mathrm{B})$ & $* *$ & & & & \\
\hline $10438 / 461$ & $\begin{array}{l}\text { Dendropicos goertae } \\
\text { (Statius Müller, 1776) }\end{array}$ & Gray Woodpecker & $1 / 4^{*}$ & $\mathrm{R}(\mathrm{B})$ & $* *$ & & & & $\mathrm{~T}$ \\
\hline $10444 / 461$ & $\begin{array}{l}\text { Dendropicos spodocephalus } \\
\text { (Bonaparte, } 1850 \text { ) }\end{array}$ & Gray-headed Woodpecker & $\mathrm{n} / 2^{*}$ & $\mathrm{R}(\mathrm{b})$ & $*$ & & & & \\
\hline $10451 / 462$ & $\begin{array}{l}\text { Dendropicos obsoletus } \\
\text { (Wagler, 1829) }\end{array}$ & $\begin{array}{l}\text { Brown-backed Wood- } \\
\text { pecker }\end{array}$ & $1 / 4 *$ & $\mathrm{R}(\mathrm{b})$ & $*$ & & & & \\
\hline \multicolumn{10}{|c|}{ FALCONIFORMES: Falconidae } \\
\hline $11081 / 96$ & $\begin{array}{l}\text { Polihierax semitorquatus } \\
\text { (A. Smith, 1836) }\end{array}$ & Pygmy Falcon & $\mathrm{m}^{*}$ & $\mathrm{R}(\mathrm{B})$ & $* * *$ & & & & CITES \\
\hline $11095 / 97$ & $\begin{array}{l}\text { Falco naumanni } \\
\text { Fleischer, } 1818\end{array}$ & Lesser Kestrel & $\mathrm{m}$ & Pl & $* * *$ & & & & CMS, CITES; P \\
\hline $11096 / 98$ & $\begin{array}{l}\text { Falco tinnunculus } \\
\text { Linnaeus, } 1758\end{array}$ & Eurasian Kestrel & $3 / 10^{*}$ & $\mathrm{R}(\mathrm{B})+\mathrm{Af}+\mathrm{Pl}$ & $* * *$ & & & & CITES \\
\hline $11122 / 99$ & $\begin{array}{l}\text { Falco rupicoloides } \\
\text { A. Smith, } 1829\end{array}$ & Greater Kestrel & $2 / 3 *$ & $\mathrm{R}(\mathrm{b})$ & $*$ & & & & CITES \\
\hline $11146 / 100$ & $\begin{array}{l}\text { Falco alopex } \\
\text { (Heuglin, 1861) }\end{array}$ & Fox Kestrel & $\mathrm{m}$ & $\mathrm{R}(\mathrm{B})$ & $* *$ & & SG & & CITES \\
\hline $11147 / 101$ & $\begin{array}{l}\text { Falco ardosiaceus } \\
\text { Vieillot, } 1823\end{array}$ & Gray Kestrel & $\mathrm{m}$ & $\mathrm{R}(\mathrm{b})$ & $* *$ & & & & CITES \\
\hline $11150 / 102$ & $\begin{array}{l}\text { Falco chicquera } \\
\text { Daudin, } 1800\end{array}$ & Red-necked Falcon & $1 / 3 *$ & $\mathrm{R} ?(\mathrm{~B})+\mathrm{Af}$ & $* *$ & & & & CITES \\
\hline $11156 / 104$ & $\begin{array}{l}\text { Falco amurensis } \\
\text { Radde, } 1863\end{array}$ & Amur Falcon & $\mathrm{m}$ & $\mathrm{Pl}$ & $*$ & & & & CITES \\
\hline $11157 / 105$ & $\begin{array}{l}\text { Falco eleonorae } \\
\text { Gene, } 1839\end{array}$ & Eleonora's Falcon & $\mathrm{m}$ & $\mathrm{Pl}$ & $*$ & & & & CITES \\
\hline $11158 / 106$ & $\begin{array}{l}\text { Falco concolor } \\
\text { Temminck, } 1825\end{array}$ & Sooty Falcon & $\mathrm{m}$ & Af & $*$ & & & & CITES \\
\hline $11170 / 107$ & $\begin{array}{l}\text { Falco subbuteo } \\
\text { Linnaeus, } 1758\end{array}$ & Eurasian Hobby & $\mathrm{n} / 2$ & $\mathrm{Pl}$ & $* * *$ & & & & CITES \\
\hline $11173 / 108$ & $\begin{array}{l}\text { Falco cuvierii } \\
\text { A. Smith, } 1830\end{array}$ & African Hobby & $\mathrm{m}$ & $\mathrm{R}(\mathrm{B})$ & $* *$ & & & & CITES \\
\hline $11194 / 109$ & $\begin{array}{l}\text { Falco biarmicus } \\
\text { Temminck, } 1825\end{array}$ & Lanner Falcon & $1 / 5^{*}$ & $\mathrm{R}(\mathrm{B})$ & $* * *$ & & & & CITES \\
\hline $11201 / 110$ & $\begin{array}{l}\text { Falco cherrug } \\
\text { J. E. Gray, } 1834\end{array}$ & Saker Falcon & $\mathrm{n} / 2$ & $\mathrm{Pl}$ & $* *$ & & & $\mathrm{EN}$ & CMS, CITES \\
\hline $11205 / 111$ & $\begin{array}{l}\text { Falco peregrinus } \\
\text { Tunstall, } 1771\end{array}$ & Peregrine Falcon & $2 / 18^{*}$ & $\mathrm{R}(\mathrm{B})+\mathrm{Pl}$ & $* *$ & & & & CITES; P \\
\hline $11227 / 112$ & $\begin{array}{l}\text { Falco pelegrinoides } \\
\text { Temminck, } 1829\end{array}$ & Barbary Falcon & $\mathrm{m}$ & $\mathrm{R} ?+\mathrm{Pl}$ ? & $* *$ & & & & CITES \\
\hline $11230 / 113$ & $\begin{array}{l}\text { Falco fasciinucha } \\
\text { Reichenow \& Neumann, } 1895\end{array}$ & Taita Falcon & $\mathrm{m}$ & $\mathrm{R}$ ? & $*$ & & & NT & $\mathrm{P}$ \\
\hline \multicolumn{10}{|c|}{ PSITTACIFORMES: Psittaculidae } \\
\hline $11789 / 331$ & $\begin{array}{l}\text { Agapornis pullarius } \\
\text { (Linnaeus, 1758) }\end{array}$ & Red-headed Lovebird & $1 / 2 *$ & $\mathrm{R}(\mathrm{b})$ & $*$ & & & & CITES \\
\hline $11792 / 332$ & $\begin{array}{l}\text { Agapornis taranta } \\
\text { (Stanley, 1814) }\end{array}$ & Black-winged Lovebird & $\mathrm{m}$ & $\mathrm{R}(\mathrm{B})$ & $* * *$ & $\mathrm{H}$ & $\mathrm{AH}$ & & CITES \\
\hline $11817 / 333$ & $\begin{array}{l}\text { Poicephalus meyeri } \\
\text { (Cretzschmar, 1827) }\end{array}$ & Meyer's Parrot & $1 / 6^{*}$ & $\mathrm{R}(\mathrm{b})$ & $*$ & & & & CITES \\
\hline $11829 / 334$ & $\begin{array}{l}\text { Poicephalus rufiventris } \\
\text { (Rüppell, 1845) }\end{array}$ & Red-bellied Parrot & $1 / 2^{*}$ & $\mathrm{R}(\mathrm{B})$ & $* *$ & & SM & & CITES; T \\
\hline $11835 / 335$ & $\begin{array}{l}\text { Poicephalus flavifrons } \\
\text { (Rüppell, 1845) }\end{array}$ & Yellow-fronted Parrot & $\mathrm{m}$ & $\mathrm{R}(\mathrm{B})$ & $* *$ & E H & $\mathrm{AH}$ & & CITES \\
\hline \multicolumn{10}{|c|}{ PASSERIFORMES: Pittidae } \\
\hline $12380 / 463$ & $\begin{array}{l}\text { Pitta angolensis } \\
\text { Vieillot, } 1816\end{array}$ & African Pitta & $1 / 3^{*}$ & Af & $*$ & & & & \\
\hline \multicolumn{10}{|c|}{ PASSERIFORMES: Platysteiridae } \\
\hline $17403 / 467$ & $\begin{array}{l}\text { Platysteira cyanea } \\
\text { (Statius Müller, 1776) }\end{array}$ & Brown-throated Wattle-eye & $1 / 3^{*}$ & $\mathrm{R}(\mathrm{b})$ & $* *$ & & & & \\
\hline $17453 / 464$ & $\begin{array}{l}\text { Batis orientalis } \\
\text { (Heuglin, } 1870)\end{array}$ & Gray-headed Batis & $3 / 4^{*}$ & $\mathrm{R}(\mathrm{B})$ & $* * *$ & & & & \\
\hline $17458 / 465$ & $\begin{array}{l}\text { Batis minor } \\
\text { Erlanger, } 1901\end{array}$ & Black-headed Batis & $1 / 3^{*}$ & $\mathrm{R}(\mathrm{B})$ & $* * *$ & & & & \\
\hline 17463 & $\begin{array}{l}\text { Batis perkeo } \\
\text { Neumann, } 1907\end{array}$ & Pygmy Batis & $\mathrm{m}$ & $\mathrm{R}(\mathrm{B})$ & $* *$ & & SM & & \\
\hline \multicolumn{10}{|c|}{ PASSERIFORMES: Vangidae } \\
\hline $17470 / 468$ & $\begin{array}{l}\text { Prionops plumatus } \\
\text { (Shaw, 1809) }\end{array}$ & White Helmetshrike & $3 / 5^{*}$ & $\mathrm{R}(\mathrm{B})$ & $* * *$ & & & & \\
\hline
\end{tabular}




\begin{tabular}{|c|c|c|c|c|c|c|c|c|}
\hline \multicolumn{9}{|c|}{ PASSERIFORMES: Malaconotidae } \\
\hline $17571 / 481$ & $\begin{array}{l}\text { Nilaus afer } \\
\text { (Latham, 1802) }\end{array}$ & Brubru & $1 / 9^{*}$ & $\mathrm{R}(\mathrm{B})$ & $* * *$ & & & \\
\hline $17581 / 475$ & $\begin{array}{l}\text { Dryoscopus gambensis } \\
\text { (Lichtenstein, 1823) }\end{array}$ & Northern Puffback & $1 / 4^{*}$ & $\mathrm{R}(\mathrm{B})$ & $* * *$ & & & \\
\hline $17586 / 476$ & $\begin{array}{l}\text { Dryoscopus pringlii } \\
\text { Jackson, } 1893\end{array}$ & Pringle's Puffback & $\mathrm{m}$ & $\mathrm{R}(\mathrm{b})$ & $* *$ & & SM & \\
\hline $17602 / 472$ & $\begin{array}{l}\text { Tchagra minutus } \\
\text { (Hartlaub, 1858) }\end{array}$ & Marsh Tchagra & $\mathrm{n} / 3$ & $\mathrm{R}(\mathrm{b})$ & $* *$ & & & \\
\hline $17607 / 474$ & $\begin{array}{l}\text { Tchagra senegalus } \\
\text { Linnaeus, } 1766\end{array}$ & Black-crowned Tchagra & $1 / 10^{*}$ & $\mathrm{R}(\mathrm{B})$ & $* * *$ & & & \\
\hline $17629 / 473$ & $\begin{array}{l}\text { Tchagra jamesi } \\
\text { (Shelley, 1885) }\end{array}$ & Three-streaked Tchagra & $\mathrm{n} / 2$ & $\mathrm{R}(\mathrm{B})$ & $* *$ & & SM & \\
\hline $17631 / 478$ & $\begin{array}{l}\text { Laniarius ruficeps } \\
\text { (Shelley, 1885) }\end{array}$ & Red-naped Bushshrike & $1 / 3^{*}$ & $\mathrm{R}(\mathrm{b})$ & $* *$ & & SM & \\
\hline $17646 / 479$ & $\begin{array}{l}\text { Laniarius aethiopicus } \\
\text { (J. F. Gmelin, 1788) }\end{array}$ & Tropical Boubou & $\mathrm{m}$ & $\mathrm{R}(\mathrm{B})$ & $* * *$ & & & \\
\hline $17668 / 480$ & $\begin{array}{l}\text { Laniarius erythrogaster } \\
\text { (Cretzschmar, 1829) }\end{array}$ & Black-headed Gonolek & $\mathrm{m}$ & $\mathrm{R}(\mathrm{b})$ & $*$ & & & \\
\hline $17672 / 477$ & $\begin{array}{l}\text { Laniarius funebris } \\
\text { (Hartlaub, 1863) }\end{array}$ & Slate-colored Boubou & $\mathrm{n} / 2^{*}$ & $\mathrm{R}(\mathrm{B})$ & $* * *$ & & & \\
\hline $17685 / 471$ & $\begin{array}{l}\text { Rhodophoneus cruentus } \\
\text { (Ehrenberg, 1828) }\end{array}$ & Rosy-patched Bushshrike & $1 / 4^{*}$ & $\mathrm{R}(\mathrm{B})$ & $* *$ & & SM & \\
\hline $17699 / 470$ & $\begin{array}{l}\text { Telophorus sulfureopectus } \\
\text { (Lesson, 1830) }\end{array}$ & $\begin{array}{l}\text { Sulphur-breasted Bu- } \\
\text { shshrike }\end{array}$ & $1 / 2^{*}$ & $\mathrm{R}(\mathrm{B})$ & $* * *$ & & & \\
\hline $17729 / 469$ & $\begin{array}{l}\text { Malaconotus blanchoti } \\
\text { Stephens, } 1826\end{array}$ & Gray-headed Bushshrike & $2 / 7^{*}$ & $\mathrm{R}(\mathrm{B})$ & $* * *$ & & & \\
\hline \multicolumn{9}{|c|}{ PASSERIFORMES: Campephagidae } \\
\hline $17943 / 482$ & $\begin{array}{l}\text { Coracina caesia } \\
\text { (Lichtenstein, 1823) }\end{array}$ & Gray Cuckoo-shrike & $1 / 3^{*}$ & $\mathrm{R}(\mathrm{B})$ & $* * *$ & & $\mathrm{AH}$ & \\
\hline $17947 / 483$ & $\begin{array}{l}\text { Coracina pectoralis } \\
\text { (Jardine \& Selby, 1828) }\end{array}$ & $\begin{array}{l}\text { White-breasted Cuckoo- } \\
\text { shrike }\end{array}$ & $\mathrm{m}$ & $\mathrm{R}(\mathrm{b})$ & $* *$ & & & \\
\hline $18053 / 484$ & $\begin{array}{l}\text { Campephaga flava } \\
\text { Vieillot, } 1817\end{array}$ & Black Cuckoo-shrike & $\mathrm{m}$ & $\mathrm{R}(\mathrm{b})+\mathrm{Af?}$ & $* *$ & & & \\
\hline $18055 / 485$ & $\begin{array}{l}\text { Campephaga phoenicea } \\
\text { (Latham, 1790) }\end{array}$ & $\begin{array}{l}\text { Red-shouldered Cuckoo- } \\
\text { shrike }\end{array}$ & $\mathrm{m}$ & $\mathrm{R}(\mathrm{B})$ & $* *$ & & & \\
\hline \multicolumn{9}{|c|}{ PASSERIFORMES: Laniidae } \\
\hline $18512 / 487$ & $\begin{array}{l}\text { Lanius collurio } \\
\text { Linnaeus, } 1758\end{array}$ & Red-backed Shrike & $\mathrm{m}$ & Pl & $* * *$ & & & \\
\hline $18513 / 488$ & $\begin{array}{l}\text { Lanius phoenicuroides } \\
\text { (Schalow, 1875) }\end{array}$ & Red-tailed Shrike & $\mathrm{m}^{*}$ & $\mathrm{Pl}$ & $* * *$ & & & \\
\hline $18514 / 488$ & $\begin{array}{l}\text { Lanius isabellinus } \\
\text { Ehrenberg, } 1833\end{array}$ & Isabelline Shrike & $2 / 3^{*}$ & $\mathrm{Pl}$ & $* * *$ & & & \\
\hline $18579 / 490$ & $\begin{array}{l}\text { Lanius meridionalis } \\
\text { Temminck, } 1820\end{array}$ & Southern Gray Shrike & $2 / 12^{*}$ & $\mathrm{R}(\mathrm{b})+\mathrm{Pl}$ & $* * *$ & & & \\
\hline $18593 / 489$ & $\begin{array}{l}\text { Lanius minor } \\
\text { J. F. Gmelin, } 1788\end{array}$ & Lesser Gray Shrike & $\mathrm{m}$ & $\mathrm{Pl}$ & $* *$ & & & \\
\hline $18597 / 491$ & $\begin{array}{l}\text { Lanius excubitoroides } \\
\text { Prevost \& DesMurs, } 1847\end{array}$ & Gray-backed Fiscal & $2 / 3 *$ & $\mathrm{R}(\mathrm{B})$ & $* * *$ & & & $\mathrm{~T}$ \\
\hline $18602 / 492$ & $\begin{array}{l}\text { Lanius dorsalis } \\
\text { Cabanis, } 1878\end{array}$ & Taita Fiscal & $\mathrm{m}$ & $\mathrm{R}(\mathrm{B})$ & $* *$ & & SM & \\
\hline $18603 / 493$ & $\begin{array}{l}\text { Lanius somalicus } \\
\text { Cabanis, } 1859\end{array}$ & Somali Fiscal & $\mathrm{m}$ & $\mathrm{R}(\mathrm{B})$ & $* * *$ & & SM & \\
\hline $18605 / 494$ & $\begin{array}{l}\text { Lanius humeralis } \\
\text { (Stanley, 1814) }\end{array}$ & Northern Fiscal & $\mathrm{n} / 3^{*}$ & $\mathrm{R}(\mathrm{B})$ & $* * *$ & & & $\mathrm{~T}$ \\
\hline $18622 / 496$ & $\begin{array}{l}\text { Lanius nubicus } \\
\text { Lichtenstein, } 1823\end{array}$ & Masked Shrike & $\mathrm{m}$ & $\mathrm{Pl}$ & $* * *$ & & & \\
\hline $18623 / 495$ & $\begin{array}{l}\text { Lanius senator } \\
\text { Linnaeus, } 1758\end{array}$ & Woodchat Shrike & $1 / 3^{*}$ & $\mathrm{Pl}$ & $* *$ & & & \\
\hline $18637 / 486$ & $\begin{array}{l}\text { Eurocephalus rueppelli } \\
\text { Bonaparte, } 1853\end{array}$ & White-rumped Shrike & $\mathrm{m}^{*}$ & $\mathrm{R}(\mathrm{B})$ & $* * *$ & & & \\
\hline \multicolumn{9}{|c|}{ PASSERIFORMES: Oriolidae } \\
\hline $18966 / 497$ & $\begin{array}{l}\text { Oriolus oriolus } \\
\text { (Linnaeus, 1758) }\end{array}$ & Eurasian Golden Oriole & $\mathrm{m}$ & $\mathrm{Pl}$ & $* * *$ & & & \\
\hline $18969 / 4989$ & $\begin{array}{l}\text { Oriolus auratus } \\
\text { Vieillot, } 1817\end{array}$ & African Golden Oriole & $\mathrm{n} / 2$ & Af & $* *$ & & & \\
\hline $19006 / 499$ & $\begin{array}{l}\text { Oriolus monacha } \\
\text { (J. F. Gmelin, 1789) }\end{array}$ & Dark-headed Oriole & $2 / 2^{*}$ & $\mathrm{R}(\mathrm{B})$ & $* *$ & $\mathrm{H}$ & $\mathrm{AH}$ & \\
\hline $19009 / 500$ & $\begin{array}{l}\text { Oriolus larvatus } \\
\text { Lichtenstein, } 1823\end{array}$ & $\begin{array}{l}\text { African Black-headed } \\
\text { Oriole }\end{array}$ & $1 / 6^{*}$ & $\mathrm{R}(\mathrm{B})$ & $* * *$ & & & $\mathrm{~T}$ \\
\hline \multicolumn{9}{|c|}{ PASSERIFORMES: Dicruridae } \\
\hline $19053 / 501$ & $\begin{array}{l}\text { Dicrurus adsimilis } \\
\text { (Bechstein, 1794) }\end{array}$ & Fork-tailed Drongo & $1 / 4^{*}$ & $\mathrm{R}(\mathrm{B})$ & $* * *$ & & & \\
\hline
\end{tabular}




\begin{tabular}{|c|c|c|c|c|c|c|c|c|c|}
\hline \multicolumn{10}{|c|}{ PASSERIFORMES: Monarchidae } \\
\hline $19474 / 502$ & $\begin{array}{l}\text { Terpsiphone viridis } \\
\text { (Muller, 1776) }\end{array}$ & $\begin{array}{l}\text { African Paradise-Flycat- } \\
\text { cher }\end{array}$ & $1 / 10^{*}$ & $\mathrm{R}(\mathrm{B})$ & $* * *$ & & & & $\mathrm{~T}$ \\
\hline \multicolumn{10}{|c|}{ PASSERIFORMES: Corvidae } \\
\hline $20014 / 503$ & $\begin{array}{l}\text { Zavattariornis stresemanni } \\
\text { Moltoni, } 1938\end{array}$ & Ethiopian Bush-crow & $\mathrm{m}^{*}$ & $\mathrm{R}(\mathrm{B})$ & $* * *$ & $\mathrm{OEH}$ & SM & EN & $\mathrm{P}$ \\
\hline $20033 / 504$ & $\begin{array}{l}\text { Pyrrhocorax pyrrhocorax } \\
\text { (Linnaeus, 1758) }\end{array}$ & Red-billed Chough & $1 / 8^{*}$ & $\mathrm{R}(\mathrm{B})$ & $* *$ & & & & \\
\hline $20047 / 505$ & $\begin{array}{l}\text { Ptilostomus afer } \\
\text { (Linnaeus, 1766) }\end{array}$ & Piapiac & $\mathrm{m}$ & Af & $*$ & & SG & & \\
\hline $20085 / 507$ & $\begin{array}{l}\text { Corvus capensis } \\
\text { Lichtenstein, } 1823\end{array}$ & Cape Crow & $1 / 2 *$ & $\mathrm{R}(\mathrm{B})$ & $* * *$ & & & & \\
\hline $20149 / 508$ & $\begin{array}{l}\text { Corvus albus } \\
\text { Muller, } 1776\end{array}$ & Pied Crow & $\mathrm{m}$ & $\mathrm{R}(\mathrm{B})$ & $* * *$ & & & & \\
\hline $20151 / 510$ & $\begin{array}{l}\text { Corvus edithae } \\
\text { E. L. Phillips, } 1895\end{array}$ & Somali Crow & $\mathrm{m}^{*}$ & $\mathrm{R}(\mathrm{B})$ & $* * *$ & & SM & & \\
\hline $20152 / 511$ & $\begin{array}{l}\text { Corvus rhipidurus } \\
\text { Hartert, } 1918\end{array}$ & Fan-tailed Raven & $\mathrm{m}$ & $\mathrm{R}(\mathrm{B})$ & $* * *$ & & & & \\
\hline $20154 / 512$ & $\begin{array}{l}\text { Corvus crassirostris } \\
\text { Rüppell, } 1836\end{array}$ & Thick-billed Raven & $\mathrm{m}$ & $\mathrm{R}(\mathrm{B})$ & $* * *$ & $\mathrm{H}^{+}$ & $\mathrm{AH}$ & & \\
\hline \multicolumn{10}{|c|}{ PASSERIFORMES: Alaudidae } \\
\hline $20497 / 537$ & $\begin{array}{l}\text { Mirafra cantillans } \\
\text { Blyth, } 1845\end{array}$ & Singing Bushlark & $1 / 4^{*}$ & $\mathrm{R}(\mathrm{B})$ & $* *$ & & & & \\
\hline $20511 / 539$ & $\begin{array}{l}\text { Mirafra pulpa } \\
\text { Friedmann, } 1930\end{array}$ & Friedmann's Lark & $\mathrm{m}^{*}$ & $\mathrm{R}$ ?(b) & $*$ & & SM & & \\
\hline $20516 / 541$ & $\begin{array}{l}\text { Mirafra hypermetra } \\
\text { (Reichenow, 1879) }\end{array}$ & Red-winged Lark & $2 / 4^{*}$ & $\mathrm{R}(\mathrm{B})$ & $* * *$ & & SM & & \\
\hline $20553 / 542$ & $\begin{array}{l}\text { Mirafra rufocinnamomea } \\
\text { (Salvadori, 1865) }\end{array}$ & Flappet Lark & $3 / 15^{*}$ & $\mathrm{R}(\mathrm{B})$ & $* * *$ & & & & \\
\hline $20579 / 545$ & $\begin{array}{l}\text { Mirafra gilletti } \\
\text { Sharpe, } 1895\end{array}$ & Gillett's Lark & $\mathrm{n} / 2$ & $\mathrm{R}(\mathrm{b})$ & $* *$ & $\mathrm{H}^{+}$ & SM & & \\
\hline $20583 /-$ & $\begin{array}{l}\text { Heteromirafra archeri } \\
\text { Clarke, } 1920\end{array}$ & Liben Lark & $\mathrm{m}^{*}$ & $\mathrm{R}(\mathrm{b})$ & $*$ & E H & SM & $\mathrm{CR}$ & $\mathrm{P}$ \\
\hline $20585 / 546$ & $\begin{array}{l}\text { Calendulauda poecilosterna } \\
\text { (Reichenow, 1879) }\end{array}$ & Pink-breasted Lark & $\mathrm{m}$ & $\mathrm{R}(\mathrm{B})$ & $* *$ & & SM & & \\
\hline $20605 / 543$ & $\begin{array}{l}\text { Calendulauda alopex } \\
\text { (Sharpe, 1890) }\end{array}$ & Foxy Lark & $1 / 2^{*}$ & $\mathrm{R}(\mathrm{b})$ & $* *$ & & SM & & \\
\hline $20669 / 558$ & $\begin{array}{l}\text { Eremopterix leucotis } \\
\text { (Stanley, 1814) }\end{array}$ & $\begin{array}{l}\text { Chestnut-backed Sparrow- } \\
\text { Lark }\end{array}$ & $\mathrm{n} / 5$ & $\mathrm{R}(\mathrm{B})$ & $* * *$ & & & & \\
\hline $20675 / 560$ & $\begin{array}{l}\text { Eremopterix nigriceps } \\
\text { (Gould, 1839) }\end{array}$ & $\begin{array}{l}\text { Black-crowned Sparrow- } \\
\text { Lark }\end{array}$ & $1 / 4^{*}$ & $\mathrm{R}(\mathrm{b})$ & $* *$ & & & & \\
\hline $20685 / 559$ & $\begin{array}{l}\text { Eremopterix signatus } \\
\text { (Oustalet, 1886) }\end{array}$ & $\begin{array}{l}\text { Chestnut-headed Sparrow- } \\
\text { Lark }\end{array}$ & $2 / 2^{*}$ & $\mathrm{R}(\mathrm{b})$ & $* * *$ & & SM & & \\
\hline $20695 / 550$ & $\begin{array}{l}\text { Ammomanes deserti } \\
\text { (Lichtenstein, 1823) }\end{array}$ & Desert Lark & $1 / 24 *$ & $\mathrm{R}(\mathrm{B})$ & $* *$ & & & & \\
\hline $20733 / 551$ & $\begin{array}{l}\text { Calandrella brachydactyla } \\
\text { (Leisler, 1814) }\end{array}$ & Greater Short-toed Lark & $\mathrm{n} / 8$ & $\mathrm{Pl}$ & $*$ & & & & \\
\hline $20751 / 552$ & $\begin{array}{l}\text { Calandrella blanfordi } \\
\text { (Shelley, 1902) }\end{array}$ & Blanford's Lark & $1 / 3^{*}$ & $\mathrm{R}(\mathrm{b})$ & $*$ & & & & \\
\hline $20755 / 552$ & $\begin{array}{l}\text { Calandrella erlangeri } \\
\text { (Neumann, 1906) }\end{array}$ & Erlanger's Lark & $\mathrm{m}^{*}$ & $\mathrm{R}(\mathrm{B})$ & $* * *$ & E H & $\mathrm{AH}$ & & \\
\hline $20760 / 553$ & $\begin{array}{l}\text { Calandrella somalica } \\
\text { (Sharpe, 1895) }\end{array}$ & Somali Short-toed Lark & $2 / 4^{*}$ & $\mathrm{R}(\mathrm{B})$ & $* * *$ & & SM & & \\
\hline $20804 / 554$ & $\begin{array}{l}\text { Spizocorys personata } \\
\text { Sharpe, } 1895\end{array}$ & Masked Lark & $1 / 4^{*}$ & $\mathrm{R}(\mathrm{B})$ & $*$ & & SM & & \\
\hline $20810 / 555$ & $\begin{array}{l}\text { Pseudalaemon fremantlii } \\
\text { (Lort Phillips, 1897) }\end{array}$ & Short-tailed Lark & $1 / 3^{*}$ & $\mathrm{R} ?(\mathrm{~b})+\mathrm{Af} ?$ & $* *$ & & SM & & \\
\hline $20814 / 556$ & $\begin{array}{l}\text { Galerida cristata } \\
\text { (Linnaeus, 1758) }\end{array}$ & Crested Lark & $1 / 37^{*}$ & $\mathrm{R}(\mathrm{b})$ & $* *$ & & & & \\
\hline $20854 / 557$ & $\begin{array}{l}\text { Galerida theklae } \\
\text { (Brehm, 1858) }\end{array}$ & Thekla Lark & $4 / 12^{*}$ & $\mathrm{R}(\mathrm{B})$ & $* * *$ & & & & \\
\hline \multicolumn{10}{|c|}{ PASSERIFORMES: Hirundinidae } \\
\hline $21038 / 521$ & $\begin{array}{l}\text { Riparia paludicola } \\
\text { (Vieillot, 1817) }\end{array}$ & Plain Martin & $1 / 7^{*}$ & $\mathrm{R}(\mathrm{B})$ & $* * *$ & & & & \\
\hline $21051 / 522$ & $\begin{array}{l}\text { Riparia riparia } \\
\text { (Linnaeus, 1758) }\end{array}$ & Bank Swallow & $\mathrm{n} / 5$ & $\mathrm{Pl}$ & $* * *$ & & & & \\
\hline $21065 / 523$ & $\begin{array}{l}\text { Riparia cincta } \\
\text { (Boddaert, 1783) }\end{array}$ & Banded Martin & $1 / 5^{*}$ & $\mathrm{R} ?(\mathrm{~B})+\mathrm{Af} ?$ & $* * *$ & & & & \\
\hline $21076 / 529$ & $\begin{array}{l}\text { Ptyonoprogne rupestris } \\
\text { (Scopoli, 1769) }\end{array}$ & Eurasian Crag-Martin & $\mathrm{m}$ & $\mathrm{Pl}$ & $* *$ & & & & \\
\hline $21077 / 531$ & $\begin{array}{l}\text { Ptyonoprogne fuligula } \\
\text { (M. H. K. Lichtenstein, 1842) }\end{array}$ & Rock Martin & $3 / 13^{*}$ & $\mathrm{R}(\mathrm{B})+\mathrm{Pl}$ & $* * *$ & & & & \\
\hline $21096 / 524$ & $\begin{array}{l}\text { Hirundo rustica } \\
\text { Linnaeus, } 1758\end{array}$ & Barn Swallow & $\mathrm{n} / 7$ & $\mathrm{Pl}$ & $* * *$ & & & & \\
\hline
\end{tabular}




\begin{tabular}{|c|c|c|c|c|c|c|c|c|c|}
\hline $21107 / 525$ & $\begin{array}{l}\text { Hirundo lucida } \\
\text { Hartlaub, } 1858\end{array}$ & Red-chested Swallow & $1 / 3^{*}$ & $\mathrm{R}(\mathrm{B})$ & $* * *$ & & & & \\
\hline $21111 / 526$ & $\begin{array}{l}\text { Hirundo aethiopica } \\
\text { Blanford, } 1869\end{array}$ & Ethiopian Swallow & $2 / 2 *$ & $\mathrm{R}(\mathrm{B})$ & $* * *$ & & & & \\
\hline $21117 / 527$ & $\begin{array}{l}\text { Hirundo smithii } \\
\text { Leach, } 1818\end{array}$ & Wire-tailed Swallow & $\mathrm{n} / 2$ & $\mathrm{R}(\mathrm{B})$ & $* * *$ & & & & \\
\hline $21134 / 528$ & $\begin{array}{l}\text { Hirundo megaensis } \\
\text { Benson, } 1942\end{array}$ & White-tailed Swallow & $\mathrm{m}^{*}$ & $\mathrm{R}(\mathrm{B})$ & $* *$ & $\mathrm{OEH}$ & SM & VU & $\mathrm{P}$ \\
\hline $21141 / 535$ & $\begin{array}{l}\text { Cecropis daurica } \\
\text { (Laxmann, 1769) }\end{array}$ & Red-rumped Swallow & $3 / 9 *$ & $\mathrm{R}(\mathrm{B})+\mathrm{Pl} ?$ & $* * *$ & & & & \\
\hline $21160 / 533$ & $\begin{array}{l}\text { Cecropis abyssinica } \\
\text { (Guérin-Méneville, 1843) }\end{array}$ & Lesser Striped-Swallow & $\mathrm{n} / 6^{*}$ & $\mathrm{R}(\mathrm{B})$ & $* * *$ & & & & \\
\hline $21170 / 534$ & $\begin{array}{l}\text { Cecropis senegalensis } \\
\text { (Linnaeus, 1766) }\end{array}$ & Mosque Swallow & $1 / 3 *$ & $\mathrm{R} ?(\mathrm{~B})+\mathrm{Af} ?$ & $* * *$ & & & & \\
\hline $21210 / 532$ & $\begin{array}{l}\text { Delichon urbicum } \\
\text { (Linnaeus, 1758) }\end{array}$ & Common House-Martin & $1 / 3 *$ & $\mathrm{Pl}$ & $* * *$ & & & & \\
\hline $21232 / 519$ & $\begin{array}{l}\text { Psalidoprocne pristoptera } \\
\text { (Rüppell, 1840) }\end{array}$ & Black Sawwing & $4 / 12^{*}$ & $\mathrm{R}(\mathrm{B})$ & $* * *$ & & & & \\
\hline $21247 / 520$ & $\begin{array}{l}\text { Pseudhirundo griseopyga } \\
\text { (Sundevall, 1850) }\end{array}$ & Gray-rumped Swallow & $\mathrm{n} / 2$ & Af(B) & $* * *$ & & & & \\
\hline \multicolumn{10}{|c|}{ PASSERIFORMES: Paridae } \\
\hline $21563 / 514$ & $\begin{array}{l}\text { Melaniparus guineensis } \\
\text { (Shelley, 1900) }\end{array}$ & $\begin{array}{l}\text { White-shouldered Black- } \\
\text { Tit }\end{array}$ & $\mathrm{m}^{*}$ & $\mathrm{R}(\mathrm{B})$ & $* *$ & & & & \\
\hline $21564 / 514$ & $\begin{array}{l}\text { Melaniparus leucomelas } \\
\text { (Rüppell, 1840) }\end{array}$ & White-winged Black-Tit & $\mathrm{n} / 2 *$ & $\mathrm{R}(\mathrm{B})$ & $* *$ & & & & \\
\hline $21586 / 516$ & $\begin{array}{l}\text { Melaniparus thruppi } \\
\text { (Shelley, 1885) }\end{array}$ & Somali Tit & $2 / 2 *$ & $\mathrm{R}(\mathrm{B})$ & $* *$ & & SM & & \\
\hline $21590 / 515$ & $\begin{array}{l}\text { Melaniparus leuconotus } \\
\text { (Guérin-Méneville, 1843) }\end{array}$ & White-backed Black-Tit & $\mathrm{m}$ & $\mathrm{R}(\mathrm{B})$ & $* *$ & $\mathrm{H}$ & $\mathrm{AH}$ & & \\
\hline \multicolumn{10}{|c|}{ PASSERIFORMES: Remizidae } \\
\hline $21618 / 518$ & $\begin{array}{l}\text { Anthoscopus musculus } \\
\text { (Hartlaub, 1882) }\end{array}$ & $\begin{array}{l}\text { Mouse-colored Penduline- } \\
\text { Tit }\end{array}$ & $\mathrm{m}$ & $\mathrm{R}(\mathrm{B})$ & $* *$ & & SM & & \\
\hline \multicolumn{10}{|c|}{ PASSERIFORMES: Certhiidae } \\
\hline $21871 / 649$ & $\begin{array}{l}\text { Salpornis salvadori } \\
\text { (Franklin, 1831) }\end{array}$ & African Spotted Creeper & $1 / 4^{*}$ & $\mathrm{R}(\mathrm{b})$ & $* *$ & & & & \\
\hline \multicolumn{10}{|c|}{ PASSERIFORMES: Pycnonotidae } \\
\hline $22547 / 589$ & Atimastillas flavicollis & Yellow-throated Greenbul & $1 / 5^{*}$ & $\mathrm{R}(\mathrm{B})$ & $* *$ & & & & \\
\hline $22594 / 590$ & $\begin{array}{l}\text { Phyllastrephus strepitans } \\
\text { (Reichenow, 1879) }\end{array}$ & Northern Brownbul & $\mathrm{m}$ & $\mathrm{R}(\mathrm{B})$ & $* * *$ & & & & \\
\hline $22715 / 587$ & $\begin{array}{l}\text { Pycnonotus barbatus } \\
\text { (Desfontaines, 1789) }\end{array}$ & Common Bulbul & $4 / 10^{*}$ & $\mathrm{R}(\mathrm{B})$ & $* * *$ & & & & $\mathrm{~T}$ \\
\hline \multicolumn{10}{|c|}{ PASSERIFORMES: Macrosphenidae } \\
\hline $23016 / 621$ & $\begin{array}{l}\text { Sylvietta brachyura } \\
\text { Lafresnaye, } 1839\end{array}$ & Northern Crombec & $1 / 3 *$ & $\mathrm{R}(\mathrm{b})$ & $* * *$ & & & & \\
\hline $23028 / 622$ & $\begin{array}{l}\text { Sylvietta whytii } \\
\text { (Shelley, 1894) }\end{array}$ & Red-faced Crombec & $1 / 4 *$ & $\mathrm{R}(\mathrm{B})$ & $* *$ & & & & \\
\hline $23033 / 624$ & $\begin{array}{l}\text { Sylvietta isabellina } \\
\text { (Elliot, 1897) }\end{array}$ & Somali Crombec & $\mathrm{m}$ & $\mathrm{R}(\mathrm{B})$ & $* *$ & & SM & & \\
\hline $23046 / 595$ & $\begin{array}{l}\text { Melocichla mentalis } \\
\text { (Fraser, 1843) }\end{array}$ & Moustached Grass-Warbler & $1 / 5^{*}$ & $\mathrm{R}(\mathrm{B})$ & $* * *$ & & & & \\
\hline \multicolumn{10}{|c|}{ PASSERIFORMES: Phylloscopidae } \\
\hline $23231 / 613$ & $\begin{array}{l}\text { Phylloscopus umbrovirens } \\
\text { (Rüppell, 1840) }\end{array}$ & Brown Woodland-Warbler & $1 / 9 *$ & $\mathrm{R}(\mathrm{B})$ & $* *$ & & $\mathrm{AH}$ & & \\
\hline $23244 / 614$ & $\begin{array}{l}\text { Phylloscopus trochilus } \\
\text { (Linnaeus, 1758) }\end{array}$ & Willow Warbler & $3 / 3 *$ & $\mathrm{Pl}$ & $* * *$ & & & & \\
\hline $23248 / 615$ & $\begin{array}{l}\text { Phylloscopus collybita } \\
\text { (Vieillot, 1817) }\end{array}$ & Common Chiffchaff & $2 / 6^{*}$ & $\mathrm{Pl}$ & $* * *$ & & & & \\
\hline $23268 / 616$ & $\begin{array}{l}\text { Phylloscopus orientalis } \\
\text { (C. L. Brehm, 1855) }\end{array}$ & Eastern Bonelli's Warbler & $\mathrm{m}$ & $\mathrm{Pl}$ & $*$ & & & & \\
\hline $23270 / 617$ & $\begin{array}{l}\text { Phylloscopus sibilatrix } \\
\text { (Bechstein, 1793) }\end{array}$ & Wood Warbler & $\mathrm{m}$ & Pl & $*$ & & & & \\
\hline \multicolumn{10}{|c|}{ PASSERIFORMES: Acrocephalidae } \\
\hline $23469 / 607$ & $\begin{array}{l}\text { Iduna pallida } \\
\text { (Hemprich \& Ehrenberg, 1833) }\end{array}$ & $\begin{array}{l}\text { Eastern Olivaceous } \\
\text { Warbler }\end{array}$ & $2 / 4 *$ & $\mathrm{Pl}$ & $* * *$ & & & & \\
\hline $23476 / 611$ & $\begin{array}{l}\text { Iduna natalensis } \\
\text { (Smith, 1847) }\end{array}$ & African Yellow Warbler & $1 / 4 *$ & $\mathrm{R}(\mathrm{b})$ & $* *$ & & & & \\
\hline $23483 / 608$ & $\begin{array}{l}\text { Hippolais languida } \\
\text { (Ehrenberg, 1833) }\end{array}$ & Upcher's Warbler & $\mathrm{m}$ & $\mathrm{Pl}$ & $* * *$ & & & & \\
\hline $23484 / 609$ & $\begin{array}{l}\text { Hippolais olivetorum } \\
\text { (Strickland, 1837) }\end{array}$ & Olive-tree Warbler & $\mathrm{m}$ & $\mathrm{Pl}$ & $*$ & & & & \\
\hline
\end{tabular}




\begin{tabular}{|c|c|c|c|c|c|c|c|c|}
\hline $23486 / 610$ & $\begin{array}{l}\text { Hippolais icterina } \\
\text { (Vieillot, 1817) }\end{array}$ & Icterine Warbler & $\mathrm{m}$ & Pl & $* *$ & & \multirow{8}{*}{$\mathrm{EN}$} & \multirow{8}{*}{ CMS } \\
\hline $23496 / 603$ & $\begin{array}{l}\text { Acrocephalus schoenobaenus } \\
\text { (Linnaeus, 1758) }\end{array}$ & Sedge Warbler & $\mathrm{m}$ & $\mathrm{Pl}$ & $* * *$ & & & \\
\hline $23506 / 605$ & $\begin{array}{l}\text { Acrocephalus palustris } \\
\text { (Bechstein, 1798) }\end{array}$ & Marsh Warbler & $\mathrm{m}$ & Pl & $* * *$ & & & \\
\hline $23507 / 604$ & $\begin{array}{l}\text { Acrocephalus scirpaceus } \\
\text { (Hermann, 1804) }\end{array}$ & Eurasian Reed-Warbler & $\mathrm{n} / 2$ & Pl & $* * *$ & & & \\
\hline $23511 / 604$ & $\begin{array}{l}\text { Acrocephalus baeticatus } \\
\text { (Vieillot, 1817) }\end{array}$ & African Reed-Warbler & $1 / 6^{*}$ & $\mathrm{R}(\mathrm{b})$ & $* *$ & & & \\
\hline $23519 / 599$ & $\begin{array}{l}\text { Acrocephalus griseldis } \\
\text { (Hartlaub, 1891) }\end{array}$ & Basra Reed-Warbler & $\mathrm{m}$ & $\mathrm{Pl}$ & $* *$ & & & \\
\hline $23520 / 600$ & $\begin{array}{l}\text { Acrocephalus gracilirostris } \\
\text { (Hartlaub, 1864) }\end{array}$ & Lesser Swamp-Warbler & $2 / 8^{*}$ & $\mathrm{R}(\mathrm{B})$ & $* * *$ & & & \\
\hline $23538 / 601$ & $\begin{array}{l}\text { Acrocephalus arundinaceus } \\
\text { (Linnaeus, 1758) }\end{array}$ & Great Reed-Warbler & $2 / 2^{*}$ & $\mathrm{Pl}$ & $* *$ & & & \\
\hline \multicolumn{9}{|c|}{ PASSERIFORMES: Locustellidae } \\
\hline $23653 / 591$ & $\begin{array}{l}\text { Schoenicola brevirostris } \\
\text { (Sundevall, 1850) }\end{array}$ & Fan-tailed Grassbird & $1 / 2 *$ & $\mathrm{R} ?(\mathrm{~b})+\mathrm{Af} ?$ & $*$ & & & \\
\hline $23674 / 594$ & $\begin{array}{l}\text { Bradypterus cinnamomeus } \\
\text { (Rüppell, 1840) }\end{array}$ & $\begin{array}{l}\text { Cinnamon Bracken- } \\
\text { Warbler }\end{array}$ & $\mathrm{n} / 4$ & $\mathrm{R}(\mathrm{B})$ & $* * *$ & $\mathrm{AH}$ & & \\
\hline $23684 / 592$ & $\begin{array}{l}\text { Bradypterus baboecala } \\
\text { (Vieillot, 1817) }\end{array}$ & Little Rush-Warbler & $2 / 10$ & $\mathrm{R}(\mathrm{B})$ & $* * *$ & & & \\
\hline $23698 / 593$ & $\begin{array}{l}\text { Bradypterus alfredi } \\
\text { Hartlaub, } 1890\end{array}$ & Bamboo Warbler & $\mathrm{n} / 2$ & Af? & $*$ & $\mathrm{AH}$ & & \\
\hline $23717 / 597$ & $\begin{array}{l}\text { Locustella fluviatilis } \\
\text { (Wolf, 1810) }\end{array}$ & Eurasian River Warbler & $\mathrm{m}$ & Pl & $* *$ & & & \\
\hline $23718 / 598$ & $\begin{array}{l}\text { Locustella luscinioides } \\
\text { (Savi, 1824) }\end{array}$ & Savi's Warbler & $3 / 3^{*}$ & Pl & $* *$ & & & \\
\hline $23727 / 596$ & $\begin{array}{l}\text { Locustella naevia } \\
\text { (Boddaert, 1783) }\end{array}$ & $\begin{array}{l}\text { Common Grasshopper- } \\
\text { Warbler }\end{array}$ & $1 / 4^{*}$ & Pl & $*$ & & & \\
\hline \multicolumn{9}{|c|}{ PASSERIFORMES: Cisticolidae } \\
\hline $23849 / 583$ & $\begin{array}{l}\text { Apalis flavida } \\
\text { (Strickland, 1852) }\end{array}$ & Yellow-breasted Apalis & $3 / 9^{*}$ & $\mathrm{R}(\mathrm{B})$ & $* * *$ & & & \\
\hline $23919 / 585$ & $\begin{array}{l}\text { Camaroptera brachyura } \\
\text { (Vieillot, 1820) }\end{array}$ & Green-backed Camaroptera & $1 / 17^{*}$ & $\mathrm{R}(\mathrm{B})$ & $* * *$ & & & \\
\hline $23947 / 584$ & $\begin{array}{l}\text { Urorhipis rufifrons } \\
\text { (Rüppell, 1840) }\end{array}$ & Red-fronted Warbler & $1 / 3^{*}$ & $\mathrm{R}(\mathrm{B})$ & $* *$ & & & \\
\hline $23963 / 586$ & $\begin{array}{l}\text { Calamonastes simplex } \\
\text { (Cabanis, 1878) }\end{array}$ & Gray Wren-Warbler & $\mathrm{m}$ & $\mathrm{R}(\mathrm{b})$ & $* * *$ & SM & & \\
\hline $23993 / 561$ & $\begin{array}{l}\text { Cisticola erythrops } \\
\text { (Hartlaub, 1857) }\end{array}$ & Red-faced Cisticola & $2 / 6^{*}$ & $\mathrm{R}(\mathrm{B})$ & $* *$ & & & \\
\hline $24001 / 562$ & $\begin{array}{l}\text { Cisticola cantans } \\
\text { (Heuglin, 1869) }\end{array}$ & Singing Cisticola & $\mathrm{n} / 7 *$ & $\mathrm{R}(\mathrm{B})$ & $* *$ & & & \\
\hline $24041 / 564$ & $\begin{array}{l}\text { Cisticola bodessa } \\
\text { Mearns, } 1913\end{array}$ & Boran Cisticola & $2 / 2^{*}$ & $\mathrm{R}(\mathrm{B})$ & $* * *$ & SM & & \\
\hline $24044 / 563$ & $\begin{array}{l}\text { Cisticola chiniana } \\
\text { (A. Smith, 1843) }\end{array}$ & Rattling Cisticola & $1 / 17^{*}$ & $\mathrm{R}(\mathrm{B})$ & $* * *$ & & & \\
\hline $24062 / 565$ & $\begin{array}{l}\text { Cisticola cinereolus } \\
\text { Salvadori, } 1888\end{array}$ & Ashy Cisticola & $2 / 2^{*}$ & $\mathrm{R}(\mathrm{b})$ & $* * *$ & SM & & \\
\hline $24065 / 569$ & $\begin{array}{l}\text { Cisticola ruficeps } \\
\text { (Cretzschmar, 1830) }\end{array}$ & Red-pate Cisticola & $1 / 3^{*}$ & $\mathrm{R}(\mathrm{b})$ & $*$ & SG & & \\
\hline $24096 / 566$ & $\begin{array}{l}\text { Cisticola galactotes } \\
\text { (Temminck, 1821) }\end{array}$ & Winding Cisticola & $2 / 11^{*}$ & $\mathrm{R}(\mathrm{B})$ & $* * *$ & & & \\
\hline $24122 / 567$ & $\begin{array}{l}\text { Cisticola robustus } \\
\text { (Rüppell, 1845) }\end{array}$ & Stout Cisticola & $3 / 7 *$ & $\mathrm{R}(\mathrm{B})$ & $* *$ & & & \\
\hline $24132 / 568$ & $\begin{array}{l}\text { Cisticola natalensis } \\
\text { (Smith, 1843) }\end{array}$ & Croaking Cisticola & $2 / 7 *$ & $\mathrm{R}(\mathrm{B})$ & $* *$ & & & \\
\hline $24153 / 571$ & $\begin{array}{l}\text { Cisticola brachypterus } \\
\text { (Sharpe, 1870) }\end{array}$ & Siffling Cisticola & $1 / 9^{*}$ & $\mathrm{R}(\mathrm{B})$ & $* * *$ & & & \\
\hline $24164 / 572$ & $\begin{array}{l}\text { Cisticola troglodytes } \\
\text { (Antinori, 1864) }\end{array}$ & Foxy Cisticola & $1 / 2 *$ & $\mathrm{R}(\mathrm{b})$ & $* *$ & SG & & \\
\hline $24167 / 570$ & $\begin{array}{l}\text { Cisticola nana } \\
\text { G. A. Fischer \& Reichenow, } 1884\end{array}$ & Tiny Cisticola & $\mathrm{m}$ & $\mathrm{R}(\mathrm{B})$ & $* *$ & SM & & \\
\hline $24168 / 573$ & $\begin{array}{l}\text { Cisticola juncidis } \\
\text { (Rafinesque, 1810) }\end{array}$ & Zitting Cisticola & $1 / 17^{*}$ & $\mathrm{R}(\mathrm{B})$ & $* * *$ & & & \\
\hline $24190 / 574$ & $\begin{array}{l}\text { Cisticola aridulus } \\
\text { Witherby, } 1900\end{array}$ & Desert Cisticola & $1 / 9^{*}$ & $\mathrm{R}(\mathrm{B})$ & $* * *$ & & & \\
\hline $24215 / 576$ & $\begin{array}{l}\text { Cisticola brunnescens } \\
\text { Heuglin, } 1862\end{array}$ & Pectoral-patch Cisticola & $1 / 6^{*}$ & $\mathrm{R}(\mathrm{B})$ & $* * *$ & & & \\
\hline $24257 / 582$ & $\begin{array}{l}\text { Phyllolais pulchella } \\
\text { (Cretzschmar, 1830) }\end{array}$ & Buff-bellied Warbler & $\mathrm{m}$ & $\mathrm{R}(\mathrm{B})$ & $* * *$ & & & \\
\hline $24381 / 578$ & $\begin{array}{l}\text { Prinia subflava } \\
\text { (J. F. Gmelin, 1789) }\end{array}$ & Tawny-flanked Prinia & $2 / 10^{*}$ & $\mathrm{R}(\mathrm{B})$ & $* * *$ & & & \\
\hline $24403 / 579$ & $\begin{array}{l}\text { Prinia somalica } \\
\text { (Elliot, 1897) }\end{array}$ & Pale Prinia & $2 / 2^{*}$ & $\mathrm{R}(\mathrm{B})$ & $* * *$ & SM & & \\
\hline
\end{tabular}




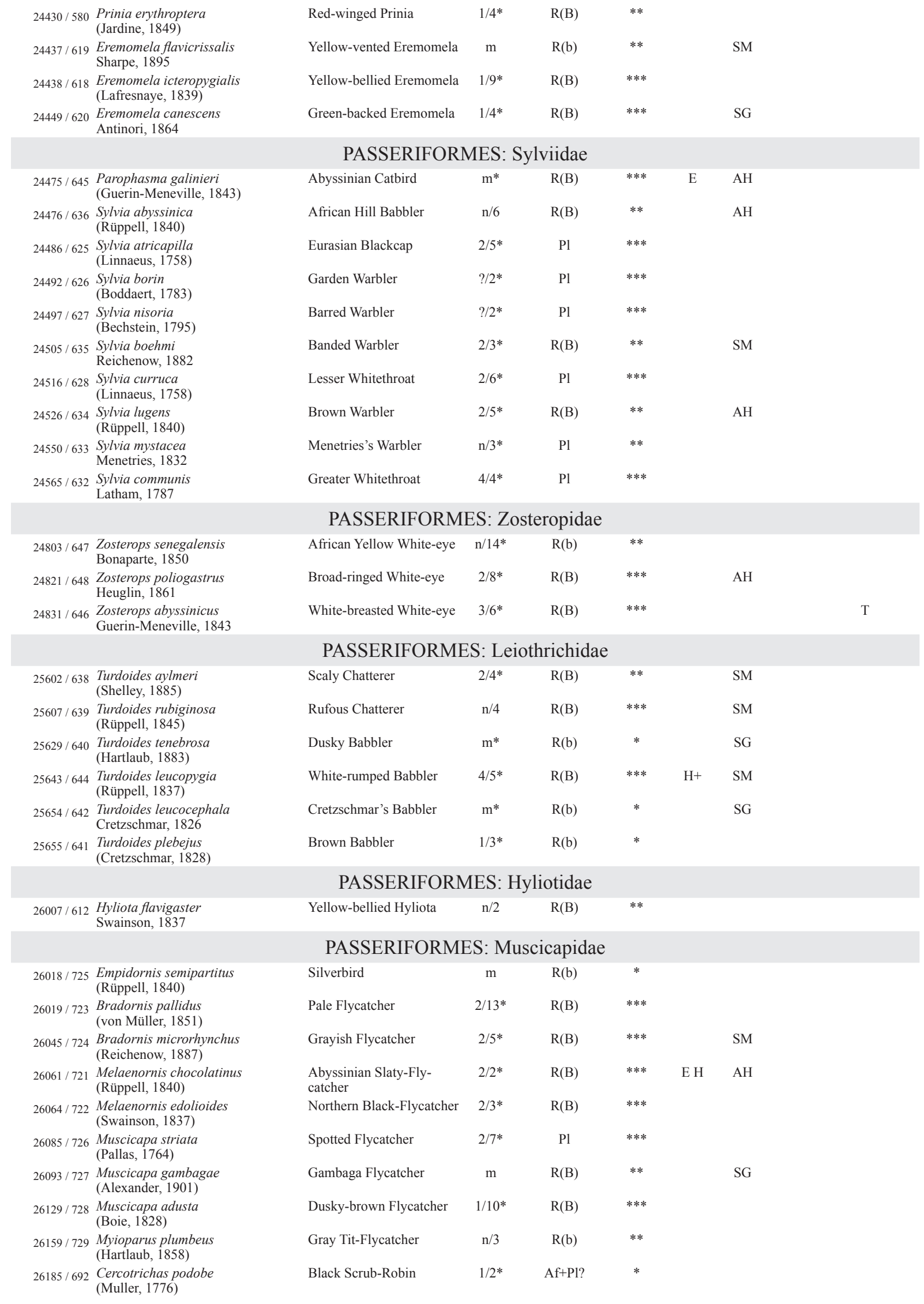




\begin{tabular}{|c|c|}
\hline $26188 / 691$ & $\begin{array}{l}\text { Cercotrichas galactotes } \\
\text { (Temminck, 1820) }\end{array}$ \\
\hline $26192 / 691$ & $\begin{array}{l}\text { Cercotrichas minor } \\
\text { (Cabanis, 1850) }\end{array}$ \\
\hline $26201 / 690$ & $\begin{array}{l}\text { Cercotrichas leucophrys } \\
\text { (Vieillot, 1817) }\end{array}$ \\
\hline $26449 / 684$ & $\begin{array}{l}\text { Cossypha semirufa } \\
\text { (Rüppell, 1837) }\end{array}$ \\
\hline $26453 / 685$ & $\begin{array}{l}\text { Cossypha heuglini } \\
\text { Hartlaub, } 1866\end{array}$ \\
\hline $26457 / 686$ & $\begin{array}{l}\text { Cossypha natalensis } \\
\text { Smith, } 1840\end{array}$ \\
\hline $26465 / 687$ & $\begin{array}{l}\text { Cossypha niveicapilla } \\
\text { (Lafresnaye, 1838) }\end{array}$ \\
\hline $26468 / 688$ & $\begin{array}{l}\text { Cossypha albicapillus } \\
\text { (Vieillot, 1818) }\end{array}$ \\
\hline $26476 / 689$ & $\begin{array}{l}\text { Cichladusa guttata } \\
\text { (Heuglin, 1862) }\end{array}$ \\
\hline $26611 / 683$ & $\begin{array}{l}\text { Irania gutturalis } \\
\text { (Guerin-Meneville, 1843) }\end{array}$ \\
\hline $26612 / 681$ & $\begin{array}{l}\text { Luscinia luscinia } \\
\text { (Linnaeus, 1758) }\end{array}$ \\
\hline $26613 / 682$ & $\begin{array}{l}\text { Luscinia megarhynchos } \\
\text { (Brehm, 1831) }\end{array}$ \\
\hline $26622 / 680$ & $\begin{array}{l}\text { Luscinia svecica } \\
\text { (Linnaeus, 1758) }\end{array}$ \\
\hline $26763 / 730$ & $\begin{array}{l}\text { Ficedula semitorquata } \\
\text { (Homeyer, 1885) }\end{array}$ \\
\hline $26812 / 694$ & $\begin{array}{l}\text { Phoenicurus phoenicurus } \\
\text { (Linnaeus, 1758) }\end{array}$ \\
\hline $26820 / 693$ & $\begin{array}{l}\text { Phoenicurus ochruros } \\
\text { (S. G. Gmelin, 1774) }\end{array}$ \\
\hline $26835 / 719$ & $\begin{array}{l}\text { Monticola rufocinereus } \\
\text { (Rüppell, 1837) }\end{array}$ \\
\hline $26852 / 718$ & $\begin{array}{l}\text { Monticola saxatilis } \\
\text { (Linnaeus, 1766) }\end{array}$ \\
\hline $26853 / 720$ & $\begin{array}{l}\text { Monticola solitarius } \\
\text { (Linnaeus, 1758) }\end{array}$ \\
\hline $26866 / 695$ & $\begin{array}{l}\text { Saxicola rubetra } \\
\text { (Linnaeus, 1758) }\end{array}$ \\
\hline $26876 /-$ & $\begin{array}{l}\text { Saxicola maurus } \\
\text { (Pallas, 1773) }\end{array}$ \\
\hline $26885 / 696$ & $\begin{array}{l}\text { Saxicola torquatus } \\
\text { (Linnaeus, 1766) }\end{array}$ \\
\hline $26940 / 714$ & $\begin{array}{l}\text { Myrmecocichla melaena } \\
\text { (Rüppell, 1837) }\end{array}$ \\
\hline $26941 / 715$ & $\begin{array}{l}\text { Myrmecocichla albifrons } \\
\text { (Rüppell, 1837) }\end{array}$ \\
\hline $26951 / 716$ & $\begin{array}{l}\text { Thamnolaea cinnamomeiventris } \\
\text { (Lafresnaye, 1836) }\end{array}$ \\
\hline $26960 / 717$ & $\begin{array}{l}\text { Thamnolaea semirufa } \\
\text { (Rüppell, 1837) }\end{array}$ \\
\hline $26977 / 709$ & $\begin{array}{l}\text { Cercomela familiaris } \\
\text { (Stephens, 1826) }\end{array}$ \\
\hline $26986 / 710$ & $\begin{array}{l}\text { Cercomela scotocerca } \\
\text { (Heuglin, 1869) }\end{array}$ \\
\hline $26995 / 711$ & $\begin{array}{l}\text { Cercomela dubia } \\
\text { (Blundell \& Lovat, 1899) }\end{array}$ \\
\hline $26996 / 712$ & $\begin{array}{l}\text { Cercomela melanura } \\
\text { (Temminck, 1824) }\end{array}$ \\
\hline $27003 / 713$ & $\begin{array}{l}\text { Cercomela sordida } \\
\text { (Rüppell, 1837) }\end{array}$ \\
\hline $27023 / 701$ & $\begin{array}{l}\text { Oenanthe phillipsi } \\
\text { (Shelley, 1885) }\end{array}$ \\
\hline $27024 / 700$ & $\begin{array}{l}\text { Oenanthe oenanthe } \\
\text { (Linnaeus, 1758) }\end{array}$ \\
\hline $27030 / 707$ & $\begin{array}{l}\text { Oenanthe lugubris } \\
\text { (Rüppell, 1837) }\end{array}$ \\
\hline $27050 / 704$ & $\begin{array}{l}\text { Oenanthe pleschanka } \\
\text { (Lepechin, 1770) }\end{array}$ \\
\hline $27052 / 705$ & $\begin{array}{l}\text { Oenanthe hispanica } \\
\text { (Linnaeus, 1758) }\end{array}$ \\
\hline $27060 / 706$ & $\begin{array}{l}\text { Oenanthe deserti } \\
\text { (Temminck, 1825) }\end{array}$ \\
\hline $27069 / 699$ & $\begin{array}{l}\text { Oenanthe isabellina } \\
\text { (Temminck, 1829) }\end{array}$ \\
\hline
\end{tabular}

\begin{tabular}{|c|c|c|c|c|}
\hline Rufous-tailed Scrub-Robin & $1 / 3 *$ & $\mathrm{Pl}$ & $* * *$ & \\
\hline African Scrub-Robin & $\mathrm{n} / 2^{*}$ & $\mathrm{R}(\mathrm{b})$ & $* * *$ & \\
\hline Red-backed Scrub-Robin & $1 / 9^{*}$ & $\mathrm{R}(\mathrm{B})$ & $* * *$ & \\
\hline Rueppell's Robin-Chat & $2 / 3^{*}$ & $\mathrm{R}(\mathrm{B})$ & $* * *$ & \\
\hline White-browed Robin-Chat & $\mathrm{n} / 3$ & $\mathrm{R}(\mathrm{b})$ & $* *$ & \\
\hline Red-capped Robin-Chat & $1 / 3^{*}$ & Af(b) & $* *$ & \\
\hline $\begin{array}{l}\text { Snowy-crowned Robin- } \\
\text { Chat }\end{array}$ & $\mathrm{n} / 2$ & $\mathrm{R}(\mathrm{B})$ & $* *$ & \\
\hline $\begin{array}{l}\text { White-crowned Robin- } \\
\text { Chat }\end{array}$ & $1 / 3^{*}$ & $\mathrm{R}(\mathrm{b})$ & * & \\
\hline Spotted Morning-Thrush & $3 / 3 *$ & $\mathrm{R}(\mathrm{B})$ & $* * *$ & \\
\hline White-throated Robin & $\mathrm{m}$ & $\mathrm{Pl}$ & $* *$ & \\
\hline Thrush Nightingale & $\mathrm{m}$ & $\mathrm{Pl}$ & $* *$ & \\
\hline Common Nightingale & $3 / 3^{*}$ & $\mathrm{Pl}$ & $* * *$ & \\
\hline Bluethroat & $3 / 12^{*}$ & $\mathrm{Pl}$ & $* *$ & \\
\hline Semi-collared Flycatcher & $\mathrm{m}$ & $\mathrm{Pl}$ & ** & \\
\hline Common Redstart & $2 / 2^{*}$ & $\mathrm{Pl}$ & $* * *$ & \\
\hline Black Redstart & $1 / 7^{*}$ & $\mathrm{Pl}$ & $*$ & \\
\hline Little Rock-Thrush & $\mathrm{n} / 2$ & $\mathrm{R}(\mathrm{B})$ & $* * *$ & \\
\hline Rufous-tailed Rock-Thrush & $\mathrm{m}$ & $\mathrm{Pl}$ & $* * *$ & \\
\hline Blue Rock-Thrush & $1 / 5^{*}$ & $\mathrm{Pl}$ & $* * *$ & \\
\hline Whinchat & $\mathrm{m}$ & $\mathrm{Pl}$ & $* * *$ & \\
\hline Siberian Stonechat & $2 / 6^{*}$ & $\mathrm{Pl}$ & $* * *$ & \\
\hline African Stonechat & $1 / 16^{*}$ & $\mathrm{R}(\mathrm{B})$ & $* * *$ & \\
\hline Rueppell's Chat & $\mathrm{m}$ & $\mathrm{R}(\mathrm{b})$ & $* *$ & $\mathrm{H}$ \\
\hline White-fronted Black-Chat & $1 / 5^{*}$ & $\mathrm{R}(\mathrm{b})$ & $*$ & \\
\hline Mocking Cliff-Chat & $2 / 8^{*}$ & $\mathrm{R}(\mathrm{B})$ & $* * *$ & \\
\hline White-winged Cliff-Chat & $\mathrm{m}$ & $\mathrm{R}(\mathrm{B})$ & $* * *$ & $\mathrm{H}$ \\
\hline Familiar Chat & $1 / 8^{*}$ & $\mathrm{R}(\mathrm{b})$ & $* *$ & \\
\hline Brown-tailed Chat & $1 / 5^{*}$ & $\mathrm{R}(\mathrm{B})$ & $* *$ & \\
\hline Sombre Chat & $\mathrm{m}^{*}$ & $\mathrm{R}(\mathrm{B})$ & $* *$ & EH \\
\hline Blackstart & $1 / 6^{*}$ & $R(B)$ & $* * *$ & \\
\hline Moorland Chat & $\mathrm{n} / 5$ & $\mathrm{R}(\mathrm{B})$ & $* * *$ & \\
\hline Somali Wheatear & $\mathrm{m}^{*}$ & $\mathrm{R}(\mathrm{b})$ & $*$ & $\mathrm{H}$ \\
\hline Northern Wheatear & $2 / 4 *$ & $\mathrm{Pl}$ & $* * *$ & \\
\hline Mourning Wheatear & $1 / 3^{*}$ & $\mathrm{R}(\mathrm{B})$ & $* * *$ & \\
\hline Pied Wheatear & $\mathrm{m}$ & Pl & $* * *$ & \\
\hline Black-eared Wheatear & $1 / 2^{*}$ & $\mathrm{Pl}$ & $* *$ & \\
\hline Desert Wheatear & $\mathrm{n} / 4$ & $\mathrm{Pl}$ & $* *$ & \\
\hline Isabelline Wheatear & $\mathrm{m}$ & $\mathrm{Pl}$ & $* * *$ & \\
\hline
\end{tabular}




\begin{tabular}{|c|c|c|c|c|c|c|c|c|}
\hline $27070 / 697$ & $\begin{array}{l}\text { Oenanthe bottae } \\
\text { (Bonaparte, 1854) }\end{array}$ & Red-breasted Wheatear & $1 / 2 *$ & $\mathrm{R}(\mathrm{B})$ & $* * *$ & & & \\
\hline $27073 / 698$ & $\begin{array}{l}\text { Oenanthe heuglini } \\
\text { (Finsch \& Hartlaub, 1870) }\end{array}$ & Heuglin's Wheatear & $\mathrm{m}^{*}$ & $\mathrm{R}(\mathrm{b})$ & $* *$ & & & \\
\hline \multicolumn{9}{|c|}{ PASSERIFORMES: Turdidae } \\
\hline $27111 / 674$ & $\begin{array}{l}\text { Geokichla piaggiae } \\
\text { (Bouvier, 1877) }\end{array}$ & Abyssinian Ground-Thrush & $\mathrm{n} / 5^{*}$ & $\mathrm{R}(\mathrm{B})$ & $* *$ & & $\mathrm{AH}$ & \\
\hline $27342 / 675$ & $\begin{array}{l}\text { Psophocichla litsitsirupa } \\
\text { (A. Smith, 1836) }\end{array}$ & Groundscraper Thrush & $1 / 4^{*}$ & $\mathrm{R}(\mathrm{B})$ & $* * *$ & & & \\
\hline $27358 / 678$ & $\begin{array}{l}\text { Turdus abyssinicus } \\
\text { J. F. Gmelin, } 1789\end{array}$ & Abyssinian Thrush & $\mathrm{n} / 6^{*}$ & $\mathrm{R}(\mathrm{B})$ & $* * *$ & & $\mathrm{AH}$ & \\
\hline $27386 / 676$ & $\begin{array}{l}\text { Turdus pelios } \\
\text { Bonaparte, } 1850\end{array}$ & African Thrush & $2 / 10^{*}$ & $\mathrm{R}(\mathrm{B})$ & $* * *$ & & & \\
\hline $27400 / 677$ & $\begin{array}{l}\text { Turdus tephronotus } \\
\text { Cabanis, } 1878\end{array}$ & African Bare-eyed Thrush & $\mathrm{m}$ & $\mathrm{R}(\mathrm{b})$ & $* *$ & & SM & \\
\hline \multicolumn{9}{|c|}{ PASSERIFORMES: Sturnidae } \\
\hline $27919 / 651$ & $\begin{array}{l}\text { Sturnus vulgaris } \\
\text { Linnaeus, } 1758\end{array}$ & European Starling & $\mathrm{n} / 12$ & $\mathrm{Pl}$ & $*$ & & & \\
\hline $27934 / 652$ & $\begin{array}{l}\text { Creatophora cinerea } \\
\text { (Meuschen, 1787) }\end{array}$ & Wattled Starling & $\mathrm{m}$ & $\mathrm{R}(\mathrm{b})+\mathrm{Af}$ & $* * *$ & & & $\mathrm{~T}$ \\
\hline $27935 / 650$ & $\begin{array}{l}\text { Pastor roseus } \\
\text { (Linnaeus, 1758) }\end{array}$ & Rosy Starling & $\mathrm{m}$ & $\mathrm{Pl}$ & $*$ & & & \\
\hline $27988 / 662$ & $\begin{array}{l}\text { Cinnyricinclus leucogaster } \\
\text { (Boddaert, 1783) }\end{array}$ & Violet-backed Starling & $2 / 3 *$ & $\mathrm{R}(\mathrm{B})+\mathrm{Af}$ & $* * *$ & & & $\mathrm{~T}$ \\
\hline $27992 / 665$ & $\begin{array}{l}\text { Onychognathus tenuirostris } \\
\text { (Rüppell, 1836) }\end{array}$ & Slender-billed Starling & $\mathrm{n} / 2^{*}$ & $\mathrm{R}(\mathrm{B})$ & $* * *$ & & $\mathrm{AH}$ & \\
\hline $27999 / 664$ & $\begin{array}{l}\text { Onychognathus morio } \\
\text { (Linnaeus, 1766) }\end{array}$ & Red-winged Starling & $1 / 2 *$ & $\mathrm{R}(\mathrm{B})$ & $* * *$ & & & \\
\hline $28012 / 668$ & $\begin{array}{l}\text { Onychognathus albirostris } \\
\text { (Rüppell, 1836) }\end{array}$ & White-billed Starling & $\mathrm{m}^{*}$ & $\mathrm{R}(\mathrm{B})$ & $* * *$ & $\mathrm{H}$ & $\mathrm{AH}$ & \\
\hline $28013 / 667$ & $\begin{array}{l}\text { Onychognathus salvadorii } \\
\text { (Sharpe, 1891) }\end{array}$ & Bristle-crowned Starling & $\mathrm{m}^{*}$ & $\mathrm{R}(\mathrm{B})$ & $* * *$ & & SM & \\
\hline $28021 / 671$ & $\begin{array}{l}\text { Speculipastor bicolor } \\
\text { Reichenow, } 1879\end{array}$ & Magpie Starling & $\mathrm{m}^{*}$ & $\mathrm{R} ?(\mathrm{~b})+\mathrm{Af}$ & $* *$ & & SM & \\
\hline $28022 / 670$ & $\begin{array}{l}\text { Pholia rabica } \\
\text { (F. J. Jackson, 1898) }\end{array}$ & Sharpe's Starling & $\mathrm{m}^{*}$ & $\mathrm{R}(\mathrm{B})$ & $* *$ & & $\mathrm{AH}$ & \\
\hline $28025 / 669$ & $\begin{array}{l}\text { Poeoptera stuhlmanni } \\
\text { (Reichenow, 1893) }\end{array}$ & Stuhlmann's Starling & $\mathrm{m}$ & $\mathrm{R}(\mathrm{b})$ & $* *$ & & $\mathrm{AH}$ & \\
\hline $28035 / 659$ & $\begin{array}{l}\text { Lamprotornis shelleyi } \\
\text { (Sharpe, 1890) }\end{array}$ & Shelley's Starling & $\mathrm{m}^{*}$ & $\mathrm{R}(\mathrm{B})+\mathrm{Af}$ & $* * *$ & & SM & \\
\hline $28037 / 656$ & $\begin{array}{l}\text { Lamprotornis purpuroptera } \\
\text { Rüppell, } 1845\end{array}$ & Rueppell's Starling & $\mathrm{n} / 2$ & $\mathrm{R}(\mathrm{B})$ & $* * *$ & & & $\mathrm{~T}$ \\
\hline $28047 / 655$ & $\begin{array}{l}\text { Lamprotornis splendidus } \\
\text { (Vieillot, 1822) }\end{array}$ & Splendid Starling & $\mathrm{n} / 4$ & $\mathrm{R}(\mathrm{b})$ & $* *$ & & & \\
\hline $28053 / 657$ & $\begin{array}{l}\text { Lamprotornis regius } \\
\text { (Reichenow, 1879) }\end{array}$ & Golden-breasted Starling & $\mathrm{m}$ & $\mathrm{R}(\mathrm{B})$ & $* * *$ & & SM & \\
\hline $28054 / 658$ & $\begin{array}{l}\text { Lamprotornis superbus } \\
\text { Rüppell, } 1845\end{array}$ & Superb Starling & $\mathrm{m}$ & $\mathrm{R}(\mathrm{B})$ & $* * *$ & & & $\mathrm{~T}$ \\
\hline $28057 / 663$ & $\begin{array}{l}\text { Lamprotornis albicapillus } \\
\text { (Blyth, 1856) }\end{array}$ & White-crowned Starling & $2 / 2^{*}$ & $\mathrm{R}(\mathrm{B})$ & $* * *$ & $\mathrm{H}+$ & SM & \\
\hline $28060 / 661$ & $\begin{array}{l}\text { Lamprotornis fischeri } \\
\text { (Reichenow, 1884) }\end{array}$ & Fischer's Starling & $\mathrm{m}$ & $\mathrm{R}(\mathrm{b})$ & $*$ & & SM & \\
\hline $28061 / 654$ & $\begin{array}{l}\text { Lamprotornis chloropterus } \\
\text { Swainson, } 1838\end{array}$ & Lesser Blue-eared Starling & $\mathrm{n} / 2$ & $\mathrm{R}(\mathrm{B})$ & $* * *$ & & SG & \\
\hline $28067 / 653$ & $\begin{array}{l}\text { Lamprotornis chalybaeus } \\
\text { Ehrenberg, } 1828\end{array}$ & Greater Blue-eared Starling & $1 / 4 *$ & $\mathrm{R}(\mathrm{B})$ & $* * *$ & & & $\mathrm{~T}$ \\
\hline \multicolumn{9}{|c|}{ PASSERIFORMES: Buphagidae } \\
\hline $28082 / 672$ & $\begin{array}{l}\text { Buphagus erythrorhynchus } \\
\text { (Stanley, 1814) }\end{array}$ & Red-billed Oxpecker & $\mathrm{m}$ & $\mathrm{R}(\mathrm{B})$ & $* * *$ & & & \\
\hline \multicolumn{9}{|c|}{ PASSERIFORMES: Nectariniidae } \\
\hline $28359 / 731$ & $\begin{array}{l}\text { Anthreptes orientalis } \\
\text { Hartlaub, } 1880\end{array}$ & $\begin{array}{l}\text { Kenya Violet-backed } \\
\text { Sunbird }\end{array}$ & $\mathrm{m}$ & $\mathrm{R}(\mathrm{B})$ & $* * *$ & & SM & \\
\hline $28370 / 732$ & $\begin{array}{l}\text { Hedydipna collaris } \\
\text { (Vieillot, 1819) }\end{array}$ & Collared Sunbird & $2 / 9 *$ & $\mathrm{R}(\mathrm{B})$ & $* * *$ & & & \\
\hline $28380 / 733$ & $\begin{array}{l}\text { Hedydipna platura } \\
\text { (Vieillot, 1819) }\end{array}$ & Pygmy Sunbird & $\mathrm{m}$ & Af & $*$ & & & \\
\hline $28381 / 734$ & $\begin{array}{l}\text { Hedydipna metallica } \\
\text { (Lichtenstein, 1823) }\end{array}$ & Nile Valley Sunbird & $\mathrm{m}$ & $\mathrm{R}(\mathrm{B})+\mathrm{Af} ?$ & $* * *$ & & & \\
\hline $28416 / 735$ & $\begin{array}{l}\text { Cyanomitra obscura } \\
\text { (Jardine, 1843) }\end{array}$ & Western Olive Sunbird & $1 / 6^{*}$ & $\mathrm{R}(\mathrm{B})$ & $* *$ & & & \\
\hline $28441 / 736$ & $\begin{array}{l}\text { Chalcomitra senegalensis } \\
\text { (Linnaeus, 1766) }\end{array}$ & Scarlet-chested Sunbird & $1 / 5^{*}$ & $\mathrm{R}(\mathrm{B})$ & $* * *$ & & & $\mathrm{~T}$ \\
\hline $28447 / 737$ & $\begin{array}{l}\text { Chalcomitra hunteri } \\
\text { (Shelley, 1889) }\end{array}$ & Hunter's Sunbird & $? / 2 *$ & $\mathrm{R}(\mathrm{b})$ & $* * *$ & & SM & \\
\hline
\end{tabular}




\begin{tabular}{|c|c|c|c|c|c|c|c|c|c|}
\hline $28493 / 738$ & $\begin{array}{l}\text { Nectarinia tacazze } \\
\text { (Stanley, 1814) }\end{array}$ & Tacazze Sunbird & $\mathrm{n} / 2^{*}$ & $\mathrm{R}(\mathrm{B})$ & $* * *$ & \multicolumn{3}{|c|}{$\mathrm{AH}$} & \multirow[t]{2}{*}{$\mathrm{T}$} \\
\hline $28500 / 739$ & $\begin{array}{l}\text { Nectarinia famosa } \\
\text { (Linnaeus, 1766) }\end{array}$ & Malachite Sunbird & $1 / 2 *$ & $\mathrm{R}(\mathrm{b})$ & $* *$ & & & & \\
\hline $28554 / 741$ & $\begin{array}{l}\text { Cinnyris pulchellus } \\
\text { (Linnaeus, 1766) }\end{array}$ & Beautiful Sunbird & $1 / 2 *$ & $\mathrm{R}(\mathrm{B})$ & $* * *$ & & & & \multirow[t]{2}{*}{$\mathrm{T}$} \\
\hline $28557 / 742$ & $\begin{array}{l}\text { Cinnyris mariquensis } \\
\text { A. Smith, } 1836\end{array}$ & Mariqua Sunbird & $1 / 3 *$ & $\mathrm{R}(\mathrm{B})$ & $* * *$ & & & & \\
\hline 28566 & $\begin{array}{l}\text { Cinnyris nectarinioides } \\
\text { Richmond, } 1897\end{array}$ & Black-bellied Sunbird & $1 / 2 *$ & $\mathrm{R}(\mathrm{b})$ & $*$ & & SM & & \\
\hline $28579 / 745$ & $\begin{array}{l}\text { Cinnyris habessinicus } \\
\text { (Hemprich \& Ehrenberg, 1828) }\end{array}$ & Shining Sunbird & $2 / 5^{*}$ & $\mathrm{R}(\mathrm{B})$ & $* * *$ & & SM & & \\
\hline $28598 / 746$ & $\begin{array}{l}\text { Cinnyris venustus } \\
\text { (Shaw, 1799) }\end{array}$ & Variable Sunbird & $2 / 5^{*}$ & $\mathrm{R}(\mathrm{B})$ & $* * *$ & & & & $\mathrm{~T}$ \\
\hline $28610 / 747$ & $\begin{array}{l}\text { Cinnyris cupreus } \\
\text { (Shaw, 1812) }\end{array}$ & Copper Sunbird & $\mathrm{n} / 2$ & $\mathrm{R}(\mathrm{b})$ & $* *$ & & & & \\
\hline \multicolumn{10}{|c|}{ PASSERIFORMES: Motacillidae } \\
\hline $28851 / 833$ & $\begin{array}{l}\text { Motacilla flava } \\
\text { Linnaeus, } 1758\end{array}$ & Western Yellow Wagtail & $6 / 12 *$ & Pl & $* * *$ & & & & \\
\hline $28875 / 834$ & $\begin{array}{l}\text { Motacilla citreola } \\
\text { Pallas, } 1776\end{array}$ & Citrine Wagtail & $\mathrm{n} / 3$ & Pl & $*$ & & & & \\
\hline $28884 / 835$ & $\begin{array}{l}\text { Motacilla cinerea } \\
\text { Tunstall, } 1771\end{array}$ & Gray Wagtail & $\mathrm{n} / 3$ & Pl & $* * *$ & & & & \\
\hline $28888 / 836$ & $\begin{array}{l}\text { Motacilla clara } \\
\text { Sharpe, } 1908\end{array}$ & Mountain Wagtail & $\mathrm{n} / 3^{*}$ & $\mathrm{R}(\mathrm{B})$ & $* * *$ & & & & \\
\hline $28892 / 837$ & $\begin{array}{l}\text { Motacilla alba } \\
\text { Linnaeus, } 1758\end{array}$ & White Wagtail & $2 / 10^{*}$ & Pl & $* * *$ & & & & \\
\hline $28908 / 838$ & $\begin{array}{l}\text { Motacilla aguimp } \\
\text { Dumont, } 1821\end{array}$ & African Pied Wagtail & $1 / 2 *$ & $\mathrm{R}(\mathrm{B})$ & $* *$ & & & & \\
\hline $28939 / 841$ & $\begin{array}{l}\text { Anthus cinnamomeus } \\
\text { Rüppell, } 1840\end{array}$ & African Pipit & $\mathrm{n} / 13 *$ & $\mathrm{R}(\mathrm{B})$ & $* * *$ & & & & \\
\hline $28961 / 843$ & $\begin{array}{l}\text { Anthus similis } \\
\text { Jerdon, } 1840\end{array}$ & Long-billed Pipit & $2 / 20^{*}$ & $\mathrm{R}(\mathrm{B})$ & $* * *$ & & & & \\
\hline $28986 / 842$ & $\begin{array}{l}\text { Anthus campestris } \\
\text { (Linnaeus, 1758) }\end{array}$ & Tawny Pipit & $\mathrm{n} / 3$ & Pl & $* *$ & & & & \\
\hline $28990 / 844$ & $\begin{array}{l}\text { Anthus leucophrys } \\
\text { Vieillot, } 1818\end{array}$ & Plain-backed Pipit & $1 / 7 *$ & $\mathrm{R}(\mathrm{B})$ & $* * *$ & & & & \\
\hline $28998 /-$ & $\begin{array}{l}\text { Anthus vaalensis } \\
\text { Shelley, } 1900\end{array}$ & Buffy Pipit & $1 / 8^{*}$ & $\mathrm{R}(\mathrm{B})$ & $* *$ & & & & \\
\hline $29030 / 845$ & $\begin{array}{l}\text { Anthus trivialis } \\
\text { (Linnaeus, 1758) }\end{array}$ & Tree Pipit & $\mathrm{n} / 2$ & Pl & $* * *$ & & & & \\
\hline $29043 / 846$ & $\begin{array}{l}\text { Anthus cervinus } \\
\text { (Pallas, 1811) }\end{array}$ & Red-throated Pipit & $\mathrm{m}$ & $\mathrm{Pl}$ & $* * *$ & & & & \\
\hline $29093 / 847$ & $\begin{array}{l}\text { Anthus caffer } \\
\text { Sundevall, } 1850\end{array}$ & Bush Pipit & $1 / 5^{*}$ & $\mathrm{R} ?+\mathrm{Af} ?$ & $*$ & & & & \\
\hline $29101 / 839$ & $\begin{array}{l}\text { Tmetothylacus tenellus } \\
\text { (Cabanis, 1878) }\end{array}$ & Golden Pipit & $\mathrm{m}$ & $\mathrm{R}(\mathrm{b})+\mathrm{Af} ?$ & $* *$ & & SM & & \\
\hline $29112 / 840$ & $\begin{array}{l}\text { Macronyx flavicollis } \\
\text { Rüppell, } 1840\end{array}$ & Abyssinian Longclaw & $\mathrm{m}$ & $\mathrm{R}(\mathrm{B})$ & $* * *$ & E H & $\mathrm{AH}$ & NT & \\
\hline \multicolumn{10}{|c|}{ PASSERIFORMES: Emberizidae } \\
\hline $31491 / 864$ & $\begin{array}{l}\text { Emberiza cineracea } \\
\text { C. L. Brehm, } 1855\end{array}$ & Cinereous Bunting & $1 / 2 *$ & Pl & $*$ & & & & \\
\hline $31494 / 865$ & $\begin{array}{l}\text { Emberiza hortulana } \\
\text { Linnaeus, } 1758\end{array}$ & Ortolan Bunting & $\mathrm{m}^{*}$ & Pl & $* * *$ & & & & \\
\hline $31499 / 867$ & $\begin{array}{l}\text { Emberiza striolata } \\
\text { (Lichtenstein, 1823) }\end{array}$ & House Bunting & $2 / 3 *$ & $\mathrm{R}(\mathrm{b})$ & $* *$ & & & & \\
\hline $31507 / 868$ & $\begin{array}{l}\text { Emberiza tahapisi } \\
\text { A. Smith, } 1836\end{array}$ & $\begin{array}{l}\text { Cinnamon-breasted Bun- } \\
\text { ting }\end{array}$ & $\mathrm{n} / 4$ & $\mathrm{R}(\mathrm{b})$ & $* * *$ & & & & \\
\hline $31551 / 869$ & $\begin{array}{l}\text { Emberiza poliopleura } \\
\text { (Salvadori, 1888) }\end{array}$ & Somali Bunting & $\mathrm{m}$ & $\mathrm{R}(\mathrm{B})$ & $* * *$ & & SM & & \\
\hline $31552 / 871$ & $\begin{array}{l}\text { Emberiza affinis } \\
\text { Heuglin, } 1867\end{array}$ & Brown-rumped Bunting & $1 / 4^{*}$ & $\mathrm{R}(\mathrm{b})$ & $* *$ & & SG & & \\
\hline \multicolumn{10}{|c|}{ PASSERIFORMES: Fringillidae } \\
\hline $32678 / 862$ & $\begin{array}{l}\text { Carduelis ankoberensis } \\
\text { (Ash, 1979) }\end{array}$ & Ankober Serin & $\mathrm{m}^{*}$ & $\mathrm{R}(\mathrm{b})$ & $*$ & $\mathrm{E} \mathrm{H}$ & $\mathrm{AH}$ & VU & $\mathrm{P}$ \\
\hline $32703 / 848$ & $\begin{array}{l}\text { Serinus flavivertex } \\
\text { (Blanford, 1869) }\end{array}$ & Yellow-crowned Canary & $\mathrm{n} / 3$ & $\mathrm{R}(\mathrm{B})$ & $* * *$ & & SM & & \\
\hline $32711 / 852$ & $\begin{array}{l}\text { Serinus leucopygius } \\
\text { (Sundevall, 1850) }\end{array}$ & White-rumped Seedeater & $\mathrm{n} / 2$ & $\mathrm{R}(\mathrm{b})$ & $* *$ & & SG & & \\
\hline $32714 / 857$ & $\begin{array}{l}\text { Serinus mozambicus } \\
\text { (Statius Muller, 1776) }\end{array}$ & Yellow-fronted Canary & $2 / 10^{*}$ & $\mathrm{R}(\mathrm{B})$ & $* * *$ & & & & \\
\hline $32725 / 849$ & $\begin{array}{l}\text { Serinus nigriceps } \\
\text { Rüppell, } 1840\end{array}$ & Abyssinian Siskin & $\mathrm{m}$ & $\mathrm{R}(\mathrm{B})$ & $* * *$ & E H & $\mathrm{AH}$ & & \\
\hline $32726 / 850$ & $\begin{array}{l}\text { Serinus citrinelloides } \\
\text { Rüppell, } 1840\end{array}$ & African Citril & $\mathrm{n} / 2^{*}$ & $\mathrm{R}(\mathrm{B})$ & $* *$ & & $\mathrm{AH}$ & & \\
\hline
\end{tabular}




\begin{tabular}{|c|c|c|c|c|c|c|c|c|c|}
\hline \\
\hline $32730 / 851$ & $\begin{array}{l}\text { Serinus hypostictus } \\
\text { (Reichenow, 1904) }\end{array}$ & Southern Citril & $1 / 2^{*}$ & $\mathrm{R}(\mathrm{b})$ & $*$ & & \multicolumn{3}{|l|}{$\mathrm{AH}$} \\
\hline $32750 / 853$ & $\begin{array}{l}\text { Serinus reichenowi } \\
\text { Salvadori, } 1888\end{array}$ & Reichenow's Seedeater & $\mathrm{m}$ & $\mathrm{R}(\mathrm{B})$ & $* * *$ & & & & \\
\hline $32751 / 854$ & $\begin{array}{l}\text { Serinus xanthopygius } \\
\text { Rüppell, } 1840\end{array}$ & Yellow-rumped Serin & $\mathrm{m}^{*}$ & $\mathrm{R}(\mathrm{b})$ & $* *$ & $\mathrm{H}$ & $\mathrm{AH}$ & & \\
\hline $32753 / 859$ & $\begin{array}{l}\text { Serinus dorsostriatus } \\
\text { (Reichenow, 1887) }\end{array}$ & White-bellied Canary & $1 / 3 *$ & $\mathrm{R}(\mathrm{B})$ & $* * *$ & & SM & & \\
\hline $32757 / 855$ & $\begin{array}{l}\text { Serinus flavigula } \\
\text { Salvadori, } 1888\end{array}$ & Yellow-throated Serin & $\mathrm{m}^{*}$ & $\mathrm{R}(\mathrm{b})$ & $*$ & E H & SM & EN & $\mathrm{P}$ \\
\hline $32758 / 856$ & $\begin{array}{l}\text { Serinus xantholaemus } \\
\text { Salvadori, } 1896\end{array}$ & Salvadori's Serin & $\mathrm{m}^{*}$ & $\mathrm{R}(\mathrm{B})$ & $* *$ & $\mathrm{EH}$ & SM & VU & $\mathrm{P}$ \\
\hline $32759 / 858$ & $\begin{array}{l}\text { Serinus donaldsoni } \\
\text { Sharpe, } 1895\end{array}$ & Northern Grosbeak-Canary & $\mathrm{m}$ & $\mathrm{R}(\mathrm{b})$ & $* *$ & & SM & & \\
\hline $32775 / 863$ & $\begin{array}{l}\text { Serinus striolatus } \\
\text { (Rüppell, 1840) }\end{array}$ & Streaky Seedeater & $\mathrm{n} / 2$ & $\mathrm{R}(\mathrm{B})$ & $* * *$ & & $\mathrm{AH}$ & & \\
\hline $32802 / 860$ & $\begin{array}{l}\text { Serinus reichardi } \\
\text { (Reichenow, 1882) }\end{array}$ & Reichard's Seedeater & $1 / 2^{*}$ & $\mathrm{R}(\mathrm{b})$ & $* *$ & & & & \\
\hline $32805 / 861$ & $\begin{array}{l}\text { Serinus tristriatus } \\
\text { Rüppell, } 1840\end{array}$ & Brown-rumped Seedeater & $\mathrm{m}$ & $\mathrm{R}(\mathrm{B})$ & $* * *$ & $\mathrm{H}$ & $\mathrm{AH}$ & & \\
\hline \multicolumn{10}{|c|}{ PASSERIFORMES: Passeridae } \\
\hline $32853 / 753$ & $\begin{array}{l}\text { Passer domesticus } \\
\text { (Linnaeus, 1758) }\end{array}$ & House Sparrow & $1 / 12^{*}$ & $\mathrm{R}(\mathrm{b})$ & $*$ & & SM & & \\
\hline $32874 / 754$ & $\begin{array}{l}\text { Passer castanopterus } \\
\text { Blyth, } 1856\end{array}$ & Somali Sparrow & $1 / 2^{*}$ & $\mathrm{R}(\mathrm{b})$ & $*$ & & SM & & \\
\hline $32893 / 755$ & $\begin{array}{l}\text { Passer shelleyi } \\
\text { Sharpe, } 1891\end{array}$ & Shelley's Rufous Sparrow & $\mathrm{m}^{*}$ & $\mathrm{R}(\mathrm{B})$ & $* *$ & & SM & & \\
\hline $32899 / 756$ & $\begin{array}{l}\text { Passer griseus } \\
\text { (Vieillot, 1817) }\end{array}$ & $\begin{array}{l}\text { Northern Gray-headed } \\
\text { Sparrow }\end{array}$ & $1 / 3^{*}$ & $\mathrm{R}(\mathrm{B})$ & $* * *$ & & & & $\mathrm{~T}$ \\
\hline $32903 / 756$ & $\begin{array}{l}\text { Passer swainsonii } \\
\text { (Rüppell, 1838) }\end{array}$ & Swainson’s Sparrow & $\mathrm{m}^{*}$ & $\mathrm{R}(\mathrm{B})$ & $* * *$ & $\mathrm{H}+$ & $\mathrm{AH}$ & & $\mathrm{T}$ \\
\hline $32904 / 756$ & $\begin{array}{l}\text { Passer gongonensis } \\
\text { (Oustalet, 1890) }\end{array}$ & Parrot-billed Sparrow & $\mathrm{m}^{*}$ & $\mathrm{R}(\mathrm{b})$ & $* *$ & & SM & & \\
\hline $32930 / 758$ & $\begin{array}{l}\text { Passer eminibey } \\
\text { (Hartlaub, 1880) }\end{array}$ & Chestnut Sparrow & $\mathrm{m}$ & $\mathrm{R}(\mathrm{B})$ & $* * *$ & & & & \\
\hline $32932 / 761$ & $\begin{array}{l}\text { Petronia pyrgita } \\
\text { (Heuglin, 1862) }\end{array}$ & Yellow-spotted Petronia & $\mathrm{n} / 2$ & $\mathrm{R}(\mathrm{B})$ & $* *$ & & SM & & \\
\hline $32943 / 760$ & $\begin{array}{l}\text { Petronia dentata } \\
\text { (Sundevall, 1850) }\end{array}$ & Bush Petronia & $\mathrm{m}$ & $\mathrm{R} ?(\mathrm{~b})+\mathrm{Af} ?$ & $* * *$ & & SG & & \\
\hline $32952 / 759$ & $\begin{array}{l}\text { Carpospiza brachydactyla } \\
\text { (Bonaparte, } 1850 \text { ) }\end{array}$ & Pale Rockfinch & $\mathrm{m}$ & $\mathrm{Pl}$ & $*$ & & & & \\
\hline \multicolumn{10}{|c|}{ PASSERIFORMES: Ploceidae } \\
\hline $32978 / 763$ & $\begin{array}{l}\text { Bubalornis niger } \\
\text { Smith, } 1836\end{array}$ & Red-billed Buffalo-Weaver & $1 / 2^{*}$ & $\mathrm{R}(\mathrm{B})$ & $* * *$ & & & & \\
\hline $32981 / 764$ & $\begin{array}{l}\text { Dinemellia dinemelli } \\
\text { (Rüppell, 1845) }\end{array}$ & $\begin{array}{l}\text { White-headed Buffalo- } \\
\text { Weaver }\end{array}$ & $\mathrm{n} / 2$ & $\mathrm{R}(\mathrm{B})$ & $* * *$ & & SM & & \\
\hline $32984 / 765$ & $\begin{array}{l}\text { Sporopipes frontalis } \\
\text { (Daudin, 1800) }\end{array}$ & Speckle-fronted Weaver & $\mathrm{n} / 2^{*}$ & $\mathrm{R}(\mathrm{B})$ & $*$ & & & & \\
\hline $32988 / 748$ & $\begin{array}{l}\text { Plocepasser mahali } \\
\text { Smith, } 1836\end{array}$ & $\begin{array}{l}\text { White-browed Sparrow- } \\
\text { Weaver }\end{array}$ & $1 / 4$ & $\mathrm{R}(\mathrm{B})$ & $* * *$ & & & & $\mathrm{~T}$ \\
\hline $32993 / 749$ & $\begin{array}{l}\text { Plocepasser superciliosus } \\
\text { (Cretzschmar, 1827) }\end{array}$ & $\begin{array}{l}\text { Chestnut-crowned Spar- } \\
\text { row-Weaver }\end{array}$ & $\mathrm{m}$ & $\mathrm{R}(\mathrm{B})$ & $* * *$ & & SG & & \\
\hline $32995 / 750$ & $\begin{array}{l}\text { Plocepasser donaldsoni } \\
\text { Sharpe, } 1895\end{array}$ & $\begin{array}{l}\text { Donaldson-Smith's Spar- } \\
\text { row-Weaver }\end{array}$ & $\mathrm{m}^{*}$ & $\mathrm{R}(\mathrm{b})$ & $*$ & & SM & & \\
\hline $32997 / 751$ & $\begin{array}{l}\text { Pseudonigrita arnaudi } \\
\text { (Bonaparte, 1850) }\end{array}$ & $\begin{array}{l}\text { Gray-headed Social- } \\
\text { Weaver }\end{array}$ & $\mathrm{n} / 2^{*}$ & $\mathrm{R}(\mathrm{B})$ & $* * *$ & & & & \\
\hline $33000 / 752$ & $\begin{array}{l}\text { Pseudonigrita cabanisi } \\
\text { (Fischer \& Reichenow, 1884) }\end{array}$ & $\begin{array}{l}\text { Black-capped Social- } \\
\text { Weaver }\end{array}$ & $\mathrm{m}$ & $\mathrm{R}(\mathrm{B})$ & $* * *$ & & SM & & \\
\hline $33026 / 782$ & $\begin{array}{l}\text { Anaplectes rubriceps } \\
\text { (Sundevall, 1850) }\end{array}$ & Red-headed Weaver & $1 / 3^{*}$ & $\mathrm{R}(\mathrm{B})$ & $* * *$ & & & & \\
\hline $33032 / 767$ & $\begin{array}{l}\text { Ploceus baglafecht } \\
\text { (Daudin, 1799) }\end{array}$ & Baglafecht Weaver & $3 / 8^{*}$ & $\mathrm{R}(\mathrm{B})$ & $* * *$ & & $\mathrm{AH}$ & & $\mathrm{T}$ \\
\hline $33046 / 768$ & $\begin{array}{l}\text { Ploceus luteolus } \\
\text { (Lichtenstein, 1823) }\end{array}$ & Little Weaver & $\mathrm{m}$ & $\mathrm{R}(\mathrm{B})$ & $* * *$ & & & & \\
\hline $33051 / 770$ & $\begin{array}{l}\text { Ploceus nigricollis } \\
\text { (Vieillot, 1805) }\end{array}$ & Black-necked Weaver & $1 / 3^{*}$ & $\mathrm{R}(\mathrm{b})$ & $* *$ & & & & \\
\hline $33057 / 769$ & $\begin{array}{l}\text { Ploceus ocularis } \\
\text { Smith, } 1839\end{array}$ & Spectacled Weaver & $1 / 3^{*}$ & $\mathrm{R}(\mathrm{B})$ & $* * *$ & & & & \\
\hline $33085 / 773$ & $\begin{array}{l}\text { Ploceus taeniopterus } \\
\text { Reichenbach, } 1863\end{array}$ & Northern Masked-Weaver & $\mathrm{m}$ & $\mathrm{R}(\mathrm{B})$ & $*$ & & & & \\
\hline $33086 / 774$ & $\begin{array}{l}\text { Ploceus intermedius } \\
\text { Rüppell, } 1845\end{array}$ & Lesser Masked-Weaver & $\mathrm{n} / 3^{*}$ & $\mathrm{R}(\mathrm{B})$ & $* * *$ & & & & $\mathrm{~T}$ \\
\hline $33097 / 775$ & $\begin{array}{l}\text { Ploceus vitellinus } \\
\text { (Lichtenstein, 1823) }\end{array}$ & Vitelline Masked-Weaver & $1 / 2 *$ & $\mathrm{R}(\mathrm{B})$ & $* * *$ & & & & \\
\hline $33104 / 772$ & $\begin{array}{l}\text { Ploceus galbula } \\
\text { Rüppell, } 1837\end{array}$ & Rueppell's Weaver & $\mathrm{m}$ & $\mathrm{R}(\mathrm{B})$ & $* * *$ & & SM & & $\mathrm{T}$ \\
\hline
\end{tabular}




\begin{tabular}{|c|c|c|c|c|c|c|c|c|}
\hline $33105 / 776$ & $\begin{array}{l}\text { Ploceus spekei } \\
\text { (Heuglin, 1861) }\end{array}$ & Speke's Weaver & $\mathrm{m}^{*}$ & $\mathrm{R}(\mathrm{B})$ & $* *$ & & SM & \\
\hline $33110 / 777$ & $\begin{array}{l}\text { Ploceus cucullatus } \\
\text { (Muller, 1776) }\end{array}$ & Village Weaver & $1 / 8^{*}$ & $\mathrm{R}(\mathrm{B})$ & $* * *$ & & & $\mathrm{~T}$ \\
\hline $33124 / 778$ & $\begin{array}{l}\text { Ploceus dichrocephalus } \\
\text { (Salvadori, 1896) }\end{array}$ & Salvadori's Weaver & $\mathrm{m}$ & $\mathrm{R}(\mathrm{B})$ & $*$ & $\mathrm{H}+$ & SM & \\
\hline $33131 / 780$ & $\begin{array}{l}\text { Ploceus rubiginosus } \\
\text { Rüppell, } 1837\end{array}$ & Chestnut Weaver & $\mathrm{n} / 2^{*}$ & $\mathrm{R}(\mathrm{B})$ & $* * *$ & & & \\
\hline $33185 / 781$ & $\begin{array}{l}\text { Pachyphantes superciliosus } \\
\text { (Shelley, 1873) }\end{array}$ & Compact Weaver & $\mathrm{m}$ & $\mathrm{R}(\mathrm{B})$ & $*$ & & & \\
\hline 33186783 & $\begin{array}{l}\text { Quelea cardinalis } \\
\text { (Hartlaub, 1880) }\end{array}$ & Cardinal Quelea & $\mathrm{m}$ & Af & $*$ & & & \\
\hline $33187 / 784$ & $\begin{array}{l}\text { Quelea erythrops } \\
\text { (Hartlaub, 1848) }\end{array}$ & Red-headed Quelea & $\mathrm{m}$ & Af & $* * *$ & & & \\
\hline $33188 / 785$ & $\begin{array}{l}\text { Quelea quelea } \\
\text { (Linnaeus, 1758) }\end{array}$ & Red-billed Quelea & $1 / 3^{*}$ & $\mathrm{R}(\mathrm{B})+\mathrm{Af}$ & $* * *$ & & & \\
\hline $33206 / 789$ & $\begin{array}{l}\text { Euplectes franciscanus } \\
\text { (Isert, 1789) }\end{array}$ & Orange Bishop & $\mathrm{m}^{*}$ & $\mathrm{R}(\mathrm{B})$ & $* * *$ & & & $\mathrm{~T}$ \\
\hline $33209 / 788$ & $\begin{array}{l}\text { Euplectes hordeaceus } \\
\text { (Linnaeus, 1758) }\end{array}$ & Black-winged Bishop & $\mathrm{m}$ & $\mathrm{R}(\mathrm{B})$ & $* * *$ & & & $\mathrm{~T}$ \\
\hline $33210 / 787$ & $\begin{array}{l}\text { Euplectes gierowii } \\
\text { Cabanis, } 1880\end{array}$ & Black Bishop & $1 / 3^{*}$ & $\mathrm{R}(\mathrm{b})$ & $* *$ & & & \\
\hline $33214 / 786$ & $\begin{array}{l}\text { Euplectes afer } \\
\text { (J. F. Gmelin, 1789) }\end{array}$ & Yellow-crowned Bishop & $2 / 3^{*}$ & $\mathrm{R}(\mathrm{B})$ & $* *$ & & & $\mathrm{~T}$ \\
\hline $33220 / 790$ & $\begin{array}{l}\text { Euplectes capensis } \\
\text { (Linnaeus, 1766) }\end{array}$ & Yellow Bishop & $1 / 6^{*}$ & $\mathrm{R}(\mathrm{B})$ & $* * *$ & & & $\mathrm{~T}$ \\
\hline $33228 / 793$ & $\begin{array}{l}\text { Euplectes albonotatus } \\
\text { (Cassin, 1848) }\end{array}$ & White-winged Widowbird & $1 / 3^{*}$ & $\mathrm{R}(\mathrm{B})$ & $* *$ & & & \\
\hline $33232 / 792$ & $\begin{array}{l}\text { Euplectes macroura } \\
\text { (J. F. Gmelin, 1789) }\end{array}$ & $\begin{array}{l}\text { Yellow-shouldered Wi- } \\
\text { dowbird }\end{array}$ & $1 / 3^{*}$ & $\mathrm{R}(\mathrm{b})$ & $* * *$ & & & $\mathrm{~T}$ \\
\hline $33236 / 794$ & $\begin{array}{l}\text { Euplectes ardens } \\
\text { (Boddaert, 1783) }\end{array}$ & Red-collared Widowbird & $1 / 3 *$ & $\mathrm{R}(\mathrm{B})$ & $* *$ & & & \\
\hline $33240 / 791$ & $\begin{array}{l}\text { Euplectes axillaris } \\
\text { (Smith, 1838) }\end{array}$ & Fan-tailed Widowbird & $2 / 5^{*}$ & $\mathrm{R}(\mathrm{B})$ & $* *$ & & & $\mathrm{~T}$ \\
\hline $33256 / 766$ & $\begin{array}{l}\text { Amblyospiza albifrons } \\
\text { (Vigors, 1831) }\end{array}$ & Grosbeak Weaver & $1 / 10^{*}$ & $\mathrm{R}(\mathrm{B})$ & $* * *$ & & & \\
\hline \multicolumn{9}{|c|}{ PASSERIFORMES: Estrildidae } \\
\hline $33297 / 809$ & $\begin{array}{l}\text { Coccopygia quartinia } \\
\text { (Bonaparte, 1850) }\end{array}$ & Yellow-bellied Waxbill & $\mathrm{n} / 3^{*}$ & $\mathrm{R}(\mathrm{B})$ & $* * *$ & & $\mathrm{AH}$ & \\
\hline $33301 / 799$ & $\begin{array}{l}\text { Mandingoa nitidula } \\
\text { (Hartlaub, 1865) }\end{array}$ & Green-backed Twinspot & $1 / 4^{*}$ & $\mathrm{R}(\mathrm{b})$ & $* * *$ & & & \\
\hline $33310 / 800$ & $\begin{array}{l}\text { Cryptospiza salvadorii } \\
\text { Reichenow, } 1892\end{array}$ & Abyssinian Crimson-wing & $\mathrm{n} / 3^{*}$ & $\mathrm{R}(\mathrm{B})$ & $* *$ & & $\mathrm{AH}$ & \\
\hline $33324 / 810$ & $\begin{array}{l}\text { Estrilda paludicola } \\
\text { Heuglin, } 1863\end{array}$ & Fawn-breasted Waxbill & $1 / 6^{*}$ & $\mathrm{R}(\mathrm{b})$ & $* * *$ & & & \\
\hline $33335 / 811$ & $\begin{array}{l}\text { Estrilda rhodopyga } \\
\text { Sundevall, } 1850\end{array}$ & Crimson-rumped Waxbill & $\mathrm{n} / 2$ & $\mathrm{R}(\mathrm{b})$ & $* * *$ & & & $\mathrm{~T}$ \\
\hline $33339 / 813$ & $\begin{array}{l}\text { Estrilda astrild } \\
\text { (Linnaeus, 1758) }\end{array}$ & Common Waxbill & $1 / 15$ & $\mathrm{R}(\mathrm{B})$ & $* * *$ & & & $\mathrm{~T}$ \\
\hline $33368 / 814$ & $\begin{array}{l}\text { Estrilda charmosyna } \\
\text { (Reichenow, 1881) }\end{array}$ & Black-cheeked Waxbill & $\mathrm{n} / 2$ & $\mathrm{R}(\mathrm{B})$ & $* *$ & & SM & \\
\hline $33387 / 806$ & $\begin{array}{l}\text { Uraeginthus bengalus } \\
\text { (Linnaeus, 1766) }\end{array}$ & Red-cheeked Cordonbleu & $\mathrm{n} / 4$ & $\mathrm{R}(\mathrm{B})$ & $* * *$ & & & $\mathrm{~T}$ \\
\hline $33394 / 808$ & $\begin{array}{l}\text { Granatina ianthinogaster } \\
\text { (Reichenow, 1879) }\end{array}$ & Purple Grenadier & $\mathrm{m}$ & $\mathrm{R}(\mathrm{B})$ & $* * *$ & & SM & $\mathrm{T}$ \\
\hline $33409 / 795$ & $\begin{array}{l}\text { Pytilia lineata } \\
\text { Heuglin, } 1863\end{array}$ & Red-billed Pytilia & $\mathrm{m}^{*}$ & $\mathrm{R}(\mathrm{B})$ & $* *$ & $\mathrm{E} \mathrm{H}+$ & SG & \\
\hline $33410 / 797$ & $\begin{array}{l}\text { Pytilia melba } \\
\text { (Linnaeus, 1758) }\end{array}$ & Green-winged Pytilia & $1 / 9^{*}$ & $\mathrm{R}(\mathrm{B})$ & $* *$ & & & \\
\hline $33421 / 796$ & $\begin{array}{l}\text { Pytilia afra } \\
\text { (J. F. Gmelin, 1789) }\end{array}$ & Orange-winged Pytilia & $\mathrm{m}$ & $\mathrm{R}(\mathrm{B})$ & $*$ & & & \\
\hline $33422 / 802$ & $\begin{array}{l}\text { Lagonosticta senegala } \\
\text { (Linnaeus, 1766) }\end{array}$ & Red-billed Firefinch & $3 / 6^{*}$ & $\mathrm{R}(\mathrm{B})$ & $* * *$ & & & $\mathrm{~T}$ \\
\hline $33429 / 801$ & $\begin{array}{l}\text { Lagonosticta rufopicta } \\
\text { (Fraser, 1843) }\end{array}$ & Bar-breasted Firefinch & $1 / 2 *$ & $\mathrm{R}(\mathrm{B})$ & $* *$ & & SG & \\
\hline $33433 / 805$ & $\begin{array}{l}\text { Lagonosticta larvata } \\
\text { (Rüppell, 1838) }\end{array}$ & Black-faced Firefinch & $\mathrm{n} / 3^{*}$ & $\mathrm{R}(\mathrm{B})$ & $* * *$ & & SG & \\
\hline $33440 / 803$ & $\begin{array}{l}\text { Lagonosticta rubricata } \\
\text { (Lichtenstein, 1823) }\end{array}$ & African Firefinch & $1 / 4^{*}$ & $\mathrm{R}(\mathrm{b})$ & $* *$ & & & \\
\hline $33446 / 804$ & $\begin{array}{l}\text { Lagonosticta rhodopareia } \\
\text { (Heuglin, 1868) }\end{array}$ & Jameson's Firefinch & $\mathrm{n} / 3$ & $\mathrm{R}(\mathrm{b})$ & $* *$ & & & \\
\hline $33454 / 798$ & $\begin{array}{l}\text { Amadina fasciata } \\
\text { (J. F. Gmelin, 1789) }\end{array}$ & Cut-throat & $1 / 4 *$ & $\mathrm{R}(\mathrm{B})$ & $* * *$ & & & $\mathrm{~T}$ \\
\hline $33462 / 815$ & $\begin{array}{l}\text { Sporaeginthus subflavus } \\
\text { (Vieillot, 1819) }\end{array}$ & Zebra Waxbill & $\mathrm{n} / 2$ & $\mathrm{R}(\mathrm{b})$ & $* *$ & & & \\
\hline $33478 / 816$ & $\begin{array}{l}\text { Ortygospiza fuscocrissa } \\
\text { Heuglin, } 1863\end{array}$ & African Quailfinch & $\mathrm{n} / 5^{*}$ & $\mathrm{R}(\mathrm{b})$ & $* *$ & & & \\
\hline
\end{tabular}




\begin{tabular}{|c|c|c|c|c|c|c|c|}
\hline $33562 / 818$ & $\begin{array}{l}\text { Odontospiza griseicapilla } \\
\text { (Delacour 1943) }\end{array}$ & Gray-headed Silverbill & $\mathrm{m}$ & $\mathrm{R}(\mathrm{b})$ & $* *$ & SM & \\
\hline $33563 / 819$ & $\begin{array}{l}\text { Spermestes cucullata } \\
\text { (Swainson, 1837) }\end{array}$ & Bronze Mannikin & $1 / 2 *$ & $\mathrm{R}(\mathrm{B})$ & $* * *$ & & $\mathrm{~T}$ \\
\hline $33566 / 820$ & $\begin{array}{l}\text { Spermestes bicolor } \\
\text { (Fraser, 1843) }\end{array}$ & Black-and-white Mannikin & $1 / 4^{*}$ & $\mathrm{R}(\mathrm{b})$ & $* * *$ & & \\
\hline $33573 / 821$ & $\begin{array}{l}\text { Spermestes fringilloides } \\
\text { (Lafresnaye, 1835) }\end{array}$ & Magpie Mannikin & $\mathrm{m}$ & $\mathrm{R} ?(\mathrm{~B})+\mathrm{Af} ?$ & $* * *$ & & \\
\hline $33575 / 817$ & $\begin{array}{l}\text { Euodice cantans } \\
\text { (Gmelin, 1789) }\end{array}$ & African Silverbill & $1 / 2 *$ & $\mathrm{R}(\mathrm{B})$ & $* * *$ & & \\
\hline \multicolumn{8}{|c|}{ PASSERIFORMES: Viduidae } \\
\hline $33685 / 826$ & $\begin{array}{l}\text { Vidua macroura } \\
\text { (Pallas, 1764) }\end{array}$ & Pin-tailed Whydah & $\mathrm{m}$ & $\mathrm{R}(\mathrm{B})$ & $* * *$ & & \\
\hline $33686 / 830$ & $\begin{array}{l}\text { Vidua orientalis } \\
\text { Heuglin, } 1870\end{array}$ & Northern Paradise-Whydah & $\mathrm{n} / 2$ & $\mathrm{R}(\mathrm{b})$ & $*$ & & \\
\hline $33689 / 831$ & $\begin{array}{l}\text { Vidua interjecta } \\
\text { (Grote, 1922) }\end{array}$ & $\begin{array}{l}\text { Exclamatory Paradise- } \\
\text { Whydah }\end{array}$ & $\mathrm{m}$ & $\mathrm{R}(\mathrm{b})$ & $*$ & SG & \\
\hline $33692 / 829$ & $\begin{array}{l}\text { Vidua paradisaea } \\
\text { (Linnaeus, 1758) }\end{array}$ & Eastern Paradise-Whydah & $\mathrm{m}$ & $\mathrm{R}(\mathrm{B})$ & $* *$ & & \\
\hline $33693 / 827$ & $\begin{array}{l}\text { Vidua hypocherina } \\
\text { Verreaux \& Verreaux, } 1856\end{array}$ & Steel-blue Whydah & $\mathrm{m}$ & $\mathrm{R}(\mathrm{B})$ & $* *$ & SM & \\
\hline $33694 / 828$ & $\begin{array}{l}\text { Vidua fischeri } \\
\text { (Reichenow, 1882) }\end{array}$ & Straw-tailed Whydah & $\mathrm{m}$ & $\mathrm{R}(\mathrm{B})$ & $* *$ & SM & \\
\hline $33696 / 822$ & $\begin{array}{l}\text { Vidua chalybeata } \\
\text { (Muller, 1776) }\end{array}$ & Village Indigobird & $1 / 6^{*}$ & $\mathrm{R}(\mathrm{B})$ & $* * *$ & & \\
\hline $33703 / 825$ & $\begin{array}{l}\text { Vidua wilsoni } \\
\text { (Hartert, 1901) }\end{array}$ & Pale-winged Indigobird & $\mathrm{m}^{*}$ & $\mathrm{R}(\mathrm{b})$ & $*$ & & \\
\hline $33706 / 823$ & $\begin{array}{l}\text { Vidua raricola } \\
\text { Payne, } 1982\end{array}$ & Jambandu Indigobird & $\mathrm{m}^{*}$ & $\mathrm{R}(\mathrm{b})$ & $*$ & & \\
\hline $33707 / 824$ & $\begin{array}{l}\text { Vidua larvaticola } \\
\text { Payne, } 1982\end{array}$ & Baka Indigobird & $\mathrm{m}^{*}$ & $\mathrm{R}(\mathrm{b})$ & $*$ & & \\
\hline $33717 / 832$ & $\begin{array}{l}\text { Anomalospiza imberbis } \\
\text { (Cabanis, 1868) }\end{array}$ & Parasitic Weaver & $\mathrm{n} / 2 *$ & $\mathrm{R} ?(\mathrm{~b})+\mathrm{Af} ?$ & $* *$ & & \\
\hline
\end{tabular}


Appendix 2. Annotations to the Checklist of Birds of Oromia. All subspecies regonized by Clements et al. (2015) that are found within the area are listed. Status of occurrence: $\mathrm{x}=$ recorded in Oromia; ? = status in Oromia questionable. Endemism: $\mathrm{O}=$ Oromia; $\mathrm{E}=$ Ethiopia; $\mathrm{H}=$ Horn of Africa (incl. Ethiopia, Eritrea, Somalia, Djibouti and Socotra); H+= Horn of Africa incl. cases where offshoots of the ranges extend to Kenya, Sudan and South Sudan. Biome restrict: $S G=$ Sudan-Guinea; $S M=$ SomaliMasai; AH = Afrotropical Highlands. Notes: N/n = North/northern; S/s = South/southern; E/e = East $/$ eastern; $\mathrm{W} / \mathrm{w}=\mathrm{West} /$ western; $\mathrm{C}=$ Central. The English and scientific names follow Clements et al. (2015). Taxon numbering, separated by slash, corresponds to the numbers given by Clements et al. (2015) and Ash \& Atkins (2009), respectively.

\begin{tabular}{|c|c|c|c|c|c|}
\hline & 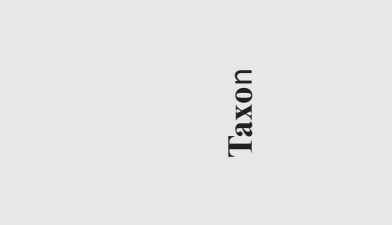 & 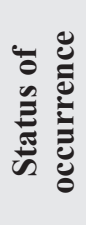 & 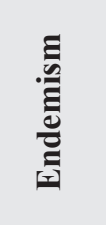 & 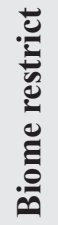 & $\begin{array}{l}\mathscr{E} \\
\stackrel{0}{0} \\
Z\end{array}$ \\
\hline $3 / 1$ & $\begin{array}{l}\text { Struthio camelus camelus } \\
\text { Linnaeus, } 1758\end{array}$ & $\mathrm{x}$ & & & $\begin{array}{l}\text { Within Oromia in far W with only one occupied 1-degree-tetrad } \\
\text { (Ash \& Atkins } 2009 \text { ). Current } S \text {. molybdophanes previously considered } \\
\text { a subspecies of } S \text {. camelus. }\end{array}$ \\
\hline $7 / 1$ & $\begin{array}{l}\text { Struthio molybdophanes } \\
\text { Reichenow, } 1883\end{array}$ & $\mathrm{x}$ & & & $\begin{array}{l}\text { Within Oromia in S and E (Ash \& Atkins 2009). Previously considered } \\
\text { a subspecies of } S \text {. camelus. }\end{array}$ \\
\hline $354 / 25$ & $\begin{array}{l}\text { Tadorna ferruginea } \\
\text { (Pallas, 1764) }\end{array}$ & $\mathrm{x}$ & & & $\begin{array}{l}\text { Isolated and only breeding population south of Sahara in Bale Mts. (Oro- } \\
\text { mia). A few scattered records elsewhere in Ethiopia and Eritrea } \\
\text { (Ash \& Atkins 2009). }\end{array}$ \\
\hline $401 / 30$ & $\begin{array}{l}\text { Anas sparsa leucostigma } \\
\text { Rüppell, } 1845\end{array}$ & $\mathrm{x}$ & & & \\
\hline $417 / 32$ & $\begin{array}{l}\text { Anas undulata ruppelli } \\
\text { Blyth, } 1855\end{array}$ & $\mathrm{x}$ & & & \\
\hline $540 / 39$ & $\begin{array}{l}\text { Netta erythrophthalma brunnea } \\
\text { (Eyton, 1838) }\end{array}$ & $\mathrm{x}$ & & & \\
\hline $820 / 2$ & $\begin{array}{l}\text { Numida meleagris meleagris } \\
\text { (Linnaeus, 1758) }\end{array}$ & $\mathrm{x}$ & & & $\begin{array}{l}\text { Two subspecies in Ethiopia and Oromia. Within Oromia N. m. meleagris } \\
\text { W of Rift Valley, } N \text {. m. somaliensis E of Rift Valley. }\end{array}$ \\
\hline $821 / 2$ & $\begin{array}{l}\text { Numida meleagris somaliensis } \\
\text { Neumann, } 1899\end{array}$ & $\mathrm{x}$ & $\mathrm{H}$ & SM & \\
\hline $844 / 4$ & $\begin{array}{l}\text { Ptilopachus petrosus petrosus } \\
\text { (J.F. Gmelin, 1789) }\end{array}$ & $\mathrm{x}$ & & & $\begin{array}{l}\text { Proposed subspecies } P . p \text {. florentiae (see map in Ash \& Atkins 2009) } \\
\text { included in P. p. petrosus. }\end{array}$ \\
\hline $1149 / 15$ & $\begin{array}{l}\text { Coturnix coturnix } \\
\text { (Linnaeus, 1758) }\end{array}$ & $\mathrm{x}$ & & & $\begin{array}{l}\text { Monotypic, subspecies coturnix and erlangeri (in Ash \& Atkins 2009) } \\
\text { not acknowledged by Clements et al. (2015). }\end{array}$ \\
\hline $1229 / 12$ & $\begin{array}{l}\text { Pternistis castaneicollis castaneicollis } \\
\text { (Salvadori, 1888) }\end{array}$ & $\mathrm{x}$ & $\mathrm{H}$ & $\mathrm{AH}$ & $\begin{array}{l}\text { Two subspecies in Ethiopia and Oromia. Within Oromia } \\
\text { P. c. castaneicollis mainly in SW, C and NE. P. c. atrifrons in S. The } \\
\text { latter nearly restricted to the Mega mountains in Oromia's extreme south }\end{array}$ \\
\hline $1230 / 12$ & $\begin{array}{l}\text { Pternistis castaneicollis atrifrons } \\
\text { (Conover, 1930) }\end{array}$ & $\mathrm{x}$ & $\mathrm{O}, \mathrm{E}, \mathrm{H}+$ & $\mathrm{AH}$ & $\begin{array}{l}\text { below } 5^{\circ} \mathrm{N} \text { (beside one single record near Moyale, Kenya). The respec- } \\
\text { tive map in Ash \& Atkins (2009) documents four occupied one-degree } \\
\text { tetrads of which one represents the Mega mountains population. The re- } \\
\text { cords from the northernmost of these tetrads (120B), however, refer to } \\
\text { nominate Chestnut-naped Francolin but not to atrifrons } \\
\text { (cf. Benson 1945), as confirmed by Benson's specimens in the British } \\
\text { Museum. Form atrifrons originally described as a separate species but } \\
\text { shows a number of distinct features that may justify a split from } \\
P \text {. castaneicollis (Töpfer et al. 2014). That would make it another en- } \\
\text { demic species to Oromia. }\end{array}$ \\
\hline $1239 / 9$ & $\begin{array}{l}\text { Pternistis squamatus } \\
\text { (Cassin, 1857) }\end{array}$ & $\mathrm{x}$ & & & $\begin{array}{l}\text { Monotypic. Form schuetti (in Ash \& Atkins 2009) not acknowledged by } \\
\text { Clements et al. (2015). }\end{array}$ \\
\hline $1246 / 10$ & $\begin{array}{l}\text { Pternistis clappertoni } \\
\text { (Children \& Vigors, 1826) }\end{array}$ & $\mathrm{x}$ & & & $\begin{array}{l}\text { Monotypic. Forms sharpi and nigrosquamatus (Ash \& Atkins 2009) not } \\
\text { acknowledged by Clements et al. (2015). }\end{array}$ \\
\hline
\end{tabular}




\begin{tabular}{|c|c|}
\hline \multirow[t]{2}{*}{$1285 / 8$} & Francolinus sephaena grantii \\
\hline & Hartlaub, 1866 \\
\hline \multirow{2}{*}{$1286 / 8$} & Francolinus sephaena spilogaster \\
\hline & Salvadori, 1888 \\
\hline \multirow{2}{*}{$1307 / 5$} & Peliperdix coqui maharao \\
\hline & W. L. Sclater, 1927 \\
\hline \multirow[t]{2}{*}{$1327 / 7$} & Scleroptila gutturalis lorti \\
\hline & (Sharpe, 1897) \\
\hline \multirow[t]{2}{*}{$1332 / 6$} & Scleroptila psilolaema psilolaema \\
\hline & (G. R. Gray, 1867) \\
\hline \multirow{2}{*}{$1503 / 47$} & Tachybaptus ruficollis capensis \\
\hline & (Salvadori, 1884) \\
\hline \multirow{2}{*}{$1721 / 48$} & Podiceps cristatus infuscatus \\
\hline & Salvadori, 1884 \\
\hline \multirow{2}{*}{$1725 / 49$} & Podiceps nigricollis gurneyi \\
\hline & (Roberts, 1919) \\
\hline \multirow{2}{*}{$2078 / 56$} & Ciconia episcopus microscelis \\
\hline & G. R. Gray, 1848 \\
\hline \multirow[t]{2}{*}{$2169 / 93$} & Phalacrocorax carbo lucidus \\
\hline & (Lichtenstein, 1823) \\
\hline \multirow[t]{2}{*}{$2247 / 87$} & Balaeniceps rex \\
\hline & Gould, 1850 \\
\hline \multirow[t]{2}{*}{$2262 / 68$} & Ixobrychus minutus minutus \\
\hline & (Linnaeus, 1766) \\
\hline \multirow[t]{2}{*}{$2263 / 68$} & Ixobrychus minutus payesii \\
\hline & (Hartlaub, 1858) \\
\hline \multirow[t]{2}{*}{$2326 / 79$} & Ardea alba melanorhynchos \\
\hline & Wagler, 1827 \\
\hline \multirow[t]{2}{*}{2334 / 80} & Mesophoyx intermedia brachyrhyncha \\
\hline & (A. E. Brehm, 1854) \\
\hline \multirow[t]{2}{*}{$2348 / 83$} & Egretta gularis schistacea \\
\hline & (Hemprich \& Ehrenberg, 1828) \\
\hline \multirow[t]{2}{*}{$2395 / 72$} & Butorides striata atricapilla \\
\hline & (Afzelius, 1804) \\
\hline \multirow[t]{2}{*}{$2512 / 62$} & Bostrychia hagedash nilotica \\
\hline & (Neumann, 1909) \\
\hline \multirow[t]{2}{*}{$2573 / 123$} & Gypaetus barbatus meridionalis \\
\hline & Keyserling \& J. H. Blasius, 1840 \\
\hline \multirow[t]{2}{*}{$2575 / 124$} & Neophron percnopterus percnopterus \\
\hline & (Linnaeus, 1758) \\
\hline \multirow[t]{2}{*}{2577124} & Neophron percnopterus ginginianus \\
\hline & (Latham, 1790) \\
\hline \multirow[t]{2}{*}{$2610 / 116$} & Aviceda cuculoides verreauxii \\
\hline & Lafresnaye, 1846 \\
\hline \multirow[t]{2}{*}{$2642 / 130$} & Torgos tracheliotos nubicus \\
\hline & (C. H. Smith, 1828) \\
\hline \multirow[t]{2}{*}{$2651 / 127$} & Gyps rueppelli erlangeri \\
\hline & Salvadori, 1908 \\
\hline
\end{tabular}

Two subspecies in Ethiopia and Oromia. Within Oromia F. s. grantii mainly in Rift Valley and S, F. s. spilogaster in easternmost fringes. However, division line between these forms unclear SM (Ash \& Atkins 2009)

Scleroptila gutturalis formerly named Francolinus levaillantoides (A. Smith, 1836). Within Oromia only S. g. lorti in S and NE (Ash \& Atkins 2009). Form archeri from S Ethiopia not acknowledged by Clements et al. (2015).

x O, E, H AH Split from S. (p.) elgonensis (E Uganda to C Kenya) considered. That would make $S$. psilolaema another endemic bird species to Ethiopia Nearly restricted to Oromia, with only two occupied 1-degree-tetrads outside (Ash \& Atkins 2009).

Records in 2004 on the Weyto river in SW Oromia (Ash \& Atkins 2009). The indicated 1-degree-tetrad is half in Oromia and half in the Southern Nations, Nationalities, and Peoples' Region.

N. p. percnopterus in Oromia. In 2010 first record of $N$. p. ginginianus in Afar region (Bulgarian Society for Protection of Birds 2010). The latter could also be expected in Oromia.

Ash \& Atkins (2009) list T. t. nubicus for Ethiopia, but according to Clements et al. (2015) this subspecies is restricted to Egypt and N Sudan while nominate tracheliotos is said to occur in Africa south of the dan while

G. r. erlangeri seems to be restricted to Horn of Africa. Subspecific affiliation of S Arabian birds uncertain. These birds mainly show the characteristics of T.t.negavensis, but not consistently (Jennings 2010). 


$\begin{array}{llc}2705 / 118 & \begin{array}{l}\text { Macheiramphus alcinus anderssoni } \\ \text { (Gurney, 1866) }\end{array} & \text { x } \\ 2762 / 161 & \begin{array}{l}\text { Aquila rapax belisarius } \\ \text { (J. Levaillant, 1850) }\end{array} & \text { x } \\ 2766 / 162 & \text { Aquila nipalensis orientalis } & \text { x } \\ & \text { Cabanis, 1854 } & \\ 2772 / 164 & \text { Aquila chrysaetos homeyeri } & \text { Severtzov, } 1888\end{array}$

$2803 / 143$

Micronisus gabar aequatorius

Clancey, 1987

$2870 / 144$

(Rüppell, 1836)

$2871 / 144$

Accipiter tachiro croizati

Desfayes, 1974

$2881 / 145$

$2995 / 150$

$3050 / 121$

Milvus migrans migrans

(Boddaert, 1783)

$3058 / 121$

(Daudin, 1800)

$3219 / 154$

(Gloger, 1833)

$3226 / 155$

Buteo oreophilus

Hartert \& Neumann, 1914

$3254 / 173$

Ardeotis arabs butleri

(Bannerman, 1930)

$3255 / 173$

Ardeotis arabs arabs

(Linnaeus, 1758)

Ardeotis kori struthiunculus

(Neumann, 1907)

$3267 / 175$

Neotis denhami denhami

(Children \& Vigors, 1826)

$3275 / 177$

Eupodotis senegalensis canicollis

(Reichenow, 1881)

$3557 / 191$

Porzana pusilla intermedia

(Hermann, 1804)

$3657 / 195$

(C. L. Brehm, 1831)

Sarothrura rufa elizabethae

Someren, 1919

Sarothrura ayresi

(Gurney, 1877)

$3733 / 198$

Podica senegalensis senegalensis

(Vieillot, 1817)
A very isolated population in the Bale Mts., discovered as late as 1988 (Clouet \& Barrau 1999), single birds at Debre Libanos in 1989 and Aseita in 2005 (Ash \& Atkins 2009). Genetically distinct, separation could date from about 200,000-300,000 years BP (Wink et al. 2004). Bale birds not described as a separate form, presumably related to $A$. $c$. homeyeri of which nearest populations are in Saudi Arabia and Oman.

\section{$\mathrm{H} \quad \mathrm{AH} \quad$ A.t. unduliventer suggested as a separate species (Louette \& Borrow 2010)}

E, $\mathrm{H} \quad \mathrm{AH}$

E, H AH Nearly endemic to Ethiopia (only two 1-degree-tetrads in Eritrea), core range in Oromia. A. r. perspicillaris considerably larger and darker than A. $r$ r rufiventris and geographically well separated from the latter (Kenya to South Africa)

M. m. migrans is a Palaearctic migrant, $M$. m. parasitus a breeding resident and intra-African migrant in Oromia.

AH Mountain Buzzard B. trizonatus of South Africa formerly considered a subspecies of $B$. oreophilus.

SG A. a. arabs in NE Oromia. A. a. butleri could be expected in easternmost Oromia, but no record so far (one occupied 1-degree-tetrad just outside, Ash \& Atkins 2009).

SM

SG Very rare. Only two occupied 1-degree-tetrads in W and SW Oromia (Ash \& Atkins 2009).

SM

P. p. obscura (Ash \& Atkins 2009) not acknowledged by

Clements et al. (2015), included in P. p. intermedia.
Breeding and maybe all-year resident in Ethiopia. Recent records from only three 1-degree-tetrads. Core areas in Sululta plain and near Addis Ababa (Ash \& Atkins 2009). Geographically isolated from birds in S Africa but preliminary genetic and isotope results indicate close relationship between Ethiopian and South African populations.

SG Birds in S Ethiopia belong possibly to P. s. senegalensis, but differ from this subspecies and may be an undescribed form (Ash \& Atkins 2009). 


\begin{tabular}{|c|c|c|c|c|c|}
\hline $3761 / 199$ & $\begin{array}{l}\text { Balearica pavonina ceciliae } \\
\text { Mitchell, } 1904\end{array}$ & $\mathrm{x}$ & & & \\
\hline $3764 / 201$ & $\begin{array}{l}\text { Bugeranus carunculatus } \\
\text { (J. F. Gmelin, 1789) }\end{array}$ & $\mathrm{x}$ & & & $\begin{array}{l}\text { Population in Ethiopia geographically isolated from other sub-equatorial } \\
\text { populations, the closest as far as Tanzania. Core area of the Ethiopian } \\
\text { population in Oromia. Comprehensive list of locations in } \\
\text { Collar \& Stuart (1985). }\end{array}$ \\
\hline $3801 / 205$ & $\begin{array}{l}\text { Burhinus oedicnemus saharae } \\
\text { (Reichenow, 1894) }\end{array}$ & ? & & & $\begin{array}{l}\text { B. o. oedicnemus is an overwintering Palaearctic visitor in Oromia. } \\
\text { B. o. saharae could be expected in Oromia and has been recorded nearby } \\
\text { in Somalia (Ash \& Atkins 2009). }\end{array}$ \\
\hline $3802 / 205$ & $\begin{array}{l}\text { Burhinus oedicnemus oedicnemus } \\
\text { (Linnaeus, 1758) }\end{array}$ & $\mathrm{x}$ & & & \\
\hline $3807 / 208$ & $\begin{array}{l}\text { Burhinus capensis maculosus } \\
\text { (Temminck, 1824) }\end{array}$ & $\mathrm{x}$ & & SG & \\
\hline $3876 / 213$ & $\begin{array}{l}\text { Vanellus crassirostris crassirostris } \\
\text { (Hartlaub, 1855) }\end{array}$ & $\mathrm{x}$ & & & Only one occupied 1-degree-tetrad in E Oromia (Ash \& Atkins 2009). \\
\hline $3883 / 215$ & $\begin{array}{l}\text { Vanellus tectus tectus } \\
\text { (Boddaert, 1783) }\end{array}$ & $\mathrm{x}$ & & & $\begin{array}{l}\text { Two subspecies in Ethiopia. Within Oromia } V . t \text {. tectus in Rift Valley and } \\
\text { Awash area. } V . t \text {. latifrons could be expected on southernmost fringes, } \\
\text { only one occupied 1-degree-tetrad on the Oromia-Kenya border, just }\end{array}$ \\
\hline $3884 / 215$ & $\begin{array}{l}\text { Vanellus tectus latifrons } \\
\text { (Reichenow, 1881) }\end{array}$ & ? & & SM & outside Oromia (Ash \& Atkins 2009). \\
\hline $3891 / 217$ & $\begin{array}{l}\text { Vanellus coronatus coronatus } \\
\text { (Boddaert, 1783) }\end{array}$ & $\mathrm{x}$ & & & $\begin{array}{l}\text { Two subspecies in Ethiopia and Oromia. Within Oromia } V . c \text {. coronatus } \\
\text { mainly in S and NE, } V . c \text {. demissus in north-easternmost fringe of Oro- } \\
\text { mia. Dividing line between the two subspecies appears uncertain (Ash \& }\end{array}$ \\
\hline $3892 / 217$ & $\begin{array}{l}\text { Vanellus coronatus demissus } \\
\text { (Friedmann, 1928) }\end{array}$ & $\mathrm{x}$ & & SM & Atkins 2009). \\
\hline $3895 / 218$ & $\begin{array}{l}\text { Vanellus senegallus major } \\
\text { (Neumann, 1914) }\end{array}$ & $\mathrm{x}$ & $\mathrm{H}$ & & $\begin{array}{l}\text { Main range of } V . \text { s. major in Ethiopia. Few occupied 1-degree-tetrads in } \\
\text { Eritrea (Ash \& Atkins 2009). }\end{array}$ \\
\hline $3926 / 230$ & $\begin{array}{l}\text { Charadrius mongolus pamirensis } \\
\text { (Richmond, 1896) }\end{array}$ & $\mathrm{x}$ & & & \\
\hline $3934 / 231$ & $\begin{array}{l}\text { Charadrius leschenaultii scythicus } \\
\text { (Severtzov, 1873) }\end{array}$ & $?$ & & & $\begin{array}{l}\text { Only one record of C. leschenaultii in Oromia, possibly referable to } \\
\text { C. l. scythicus (formerly named C. l. crassirostris; cf. } \\
\text { Ash \& Atkins 2009). }\end{array}$ \\
\hline $3963 / 224$ & $\begin{array}{l}\text { Charadrius hiaticula tundrae } \\
\text { (Lowe, 1915) }\end{array}$ & $\mathrm{x}$ & & & \\
\hline $3970 / 225$ & $\begin{array}{l}\text { Charadrius dubius curonicus } \\
\text { J.F. Gmelin, } 1789\end{array}$ & $\mathrm{x}$ & & & \\
\hline $4069 / 246$ & $\begin{array}{l}\text { Tringa totanus totanus } \\
\text { (Linnaeus, 1758) }\end{array}$ & $\mathrm{x}$ & & & $\begin{array}{l}\text { T. t. totanus and } T \text {. t. ussuriensis in Ethiopia and most likely in Oromia. } \\
\text { Both are Palaearctic passage migrants and winter visitors. Most birds are } \\
\text { probably } T \text {. } t \text {. ussurensis (Ash \& Atkins 2009). }\end{array}$ \\
\hline $4070 / 246$ & $\begin{array}{l}\text { Tringa totanus ussuriensis } \\
\text { Buturlin, } 1934\end{array}$ & $\mathrm{x}$ & & & \\
\hline $4089 / 244$ & $\begin{array}{l}\text { Numenius arquata orientalis } \\
\text { C. L. Brehm, } 1831\end{array}$ & $\mathrm{x}$ & & & \\
\hline $4147 / 260$ & $\begin{array}{l}\text { Calidris alpina alpina } \\
\text { (Linnaeus, 1758) }\end{array}$ & $\mathrm{x}$ & & & $\begin{array}{l}\text { Suggested subspecies C. a. centralis (Ash \& Atkins 2009) included in } \\
\text { C. c. alpina. }\end{array}$ \\
\hline $4221 / 237$ & $\begin{array}{l}\text { Gallinago nigripennis aequatorialis } \\
\text { Rüppell, } 1845\end{array}$ & $\mathrm{x}$ & & & \\
\hline $4243 / 203$ & $\begin{array}{l}\text { Turnix sylvaticus lepurana } \\
\text { (A. Smith, 1836) }\end{array}$ & $\mathrm{x}$ & & & \\
\hline $4314 / 267$ & $\begin{array}{l}\text { Cursorius somalensis somalensis } \\
\text { Shelley, } 1885\end{array}$ & $\mathrm{x}$ & $\mathrm{H}$ & SM & $\begin{array}{l}\text { Two subspecies in Ethiopia and Oromia. Within Oromia C. s. somalensis } \\
\text { in NE with two occupied 1-degree-tetrads, and C. s. littoralis in S with } \\
\text { four occupied 1-degree-tetrads (Ash \& Atkins 2009). Lines of subspe- }\end{array}$ \\
\hline $4315 / 267$ & $\begin{array}{l}\text { Cursorius somalensis littoralis } \\
\text { Erlanger, } 1905\end{array}$ & $\mathrm{x}$ & & SM & $\begin{array}{l}\text { cific segregation given in Ash \& Atkins' (2009) map provisional. Further } \\
\text { study needed. }\end{array}$ \\
\hline $4320 / 269$ & $\begin{array}{l}\text { Smutsornis africanus raffertyi } \\
\text { (Mearns, 1915) }\end{array}$ & $\mathrm{x}$ & $\mathrm{H}$ & SM & $\begin{array}{l}\text { Two subspecies in Ethiopia and Oromia. Within Oromia S. a. raffertyi } \\
\text { scattered mainly in Rift Valley and Middle Awash area. S. a. hartingi in } \\
\text { NE with two occupied 1-degree-tetrads (Ash \& Atkins 2009). }\end{array}$ \\
\hline $4321 / 269$ & Smutsornis africanus hartingi & $\mathrm{x}$ & $\mathrm{H}$ & SM & \\
\hline
\end{tabular}

(Sharpe, 1893) 


\begin{tabular}{|c|c|c|}
\hline $4328 / 270$ & $\begin{array}{l}\text { Rhinoptilus cinctus cinctus } \\
\text { (Heuglin, 1863) }\end{array}$ & $\mathrm{x}$ \\
\hline $4337 / 272$ & $\begin{array}{l}\text { Glareola pratincola pratincola } \\
\text { (Linnaeus, 1766) }\end{array}$ & $\mathrm{x}$ \\
\hline $4339 / 272$ & $\begin{array}{l}\text { Glareola pratincola fuelleborni } \\
\text { Neumann, } 1910\end{array}$ & ? \\
\hline $4463 / 281$ & $\begin{array}{l}\text { Chroicocephalus cirrocephalus poiocephalus } \\
\text { (Swainson, 1837) }\end{array}$ & $\mathrm{x}$ \\
\hline $4538 / 278$ & $\begin{array}{l}\text { Larus fuscus fuscus } \\
\text { Linnaeus, } 1758\end{array}$ & $\mathrm{x}$ \\
\hline $4541 / 279$ & $\begin{array}{l}\text { Larus fuscus heuglini } \\
\text { Bree, } 1876\end{array}$ & $\mathrm{x}$ \\
\hline $4613 / 294$ & $\begin{array}{l}\text { Onychoprion fuscatus nubilosus } \\
\text { (Sparrman, 1788) }\end{array}$ & $\mathrm{x}$ \\
\hline $4672 / 295$ & $\begin{array}{l}\text { Chlidonias hybrida hybrida } \\
\text { (Pallas, 1811) }\end{array}$ & $\mathrm{x}$ \\
\hline $4677 / 295$ & $\begin{array}{l}\text { Chlidonias hybrida delalandii } \\
\text { (Mathews, 1912) }\end{array}$ & ? \\
\hline $4700 /-$ & $\begin{array}{l}\text { Sterna paradisaea } \\
\text { Pontoppidan, } 1763\end{array}$ & $\mathrm{x}$ \\
\hline $4761 / 302$ & $\begin{array}{l}\text { Pterocles exustus ellioti } \\
\text { Bogdanov, } 1881\end{array}$ & $\mathrm{x}$ \\
\hline $4762 / 302$ & $\begin{array}{l}\text { Pterocles exustus olivascens } \\
\text { (Hartert, 1909) }\end{array}$ & $\mathrm{x}$ \\
\hline $4770 / 304$ & $\begin{array}{l}\text { Pterocles gutturalis saturatior } \\
\text { Hartert, } 1900\end{array}$ & $\mathrm{x}$ \\
\hline $4779 / 305$ & $\begin{array}{l}\text { Pterocles decoratus ellenbecki } \\
\text { Erlanger, } 1905\end{array}$ & $\mathrm{x}$ \\
\hline $4786 / 306$ & $\begin{array}{l}\text { Pterocles lichtensteinii lichtensteinii } \\
\text { Temminck, } 1825\end{array}$ & $\mathrm{x}$ \\
\hline $4787 / 306$ & $\begin{array}{l}\text { Pterocles lichtensteinii sukensis } \\
\text { Neumann, } 1909\end{array}$ & $\mathrm{x}$ \\
\hline $4798 / 308$ & $\begin{array}{l}\text { Columba livia } \\
\text { J. F. Gmelin, } 1789\end{array}$ & $\mathrm{x}$ \\
\hline $4853 / 313$ & $\begin{array}{l}\text { Columba larvata bronzina } \\
\text { Rüppell, } 1837\end{array}$ & $\mathrm{x}$ \\
\hline $4947 / 314$ & $\begin{array}{l}\text { Streptopelia turtur turtur } \\
\text { (Linnaeus, 1758) }\end{array}$ & $\mathrm{x}$ \\
\hline $4948 / 314$ & $\begin{array}{l}\text { Streptopelia turtur arenicola } \\
\text { (Hartert, 1894) }\end{array}$ & $\mathrm{x}$ \\
\hline $4950 / 314$ & $\begin{array}{l}\text { Streptopelia turtur rufescens } \\
\text { (C. L. Brehm, 1855) }\end{array}$ & $\mathrm{X}$ \\
\hline $4966 / 316$ & $\begin{array}{l}\text { Streptopelia roseogrisea roseogrisea } \\
\text { (Sundevall, 1857) }\end{array}$ & ? \\
\hline $4967 / 316$ & $\begin{array}{l}\text { Streptopelia roseogrisea arabica } \\
\text { (Neumann, 1904) }\end{array}$ & $\mathrm{x}$ \\
\hline $4975 / 318$ & $\begin{array}{l}\text { Streptopelia decipiens decipiens } \\
\text { (Hartlaub \& Finsch, 1870) }\end{array}$ & $\mathrm{x}$ \\
\hline $4976 / 318$ & $\begin{array}{l}\text { Streptopelia decipiens elegans } \\
\text { (Zedlitz, 1913) }\end{array}$ & $\mathrm{x}$ \\
\hline
\end{tabular}

SM R. c. cinctus in Ethiopia and Oromia. R. c. mayaudi (Ash \& Atkins 2009) not acknowledged by del Hoyo et al. (1996) and Clements et al. (2015).

Possibly two subspecies in Ethiopia and Oromia. However, only G. p. pratincola is reliably identified from Oromia, mainly in Rift Valley and Middle Awash area. Presumed G. p. fuelleborni in S (Ash \& Atkins 2009). Recent records (2011) in Sarrite plain E of Yabello, but subspecific affiliation not noticed. Clarification needed.

Two subspecies in Ethiopia and Oromia. Both are Palaearctic winter visitors and presumed passage migrants. $L$, heuglini treated as a separate species by Ash and Atkins (2009). L.f. fuscus locally very common, few species by Ash and Atkins (2009). L. f. fuscus locally very common, few
L.f. heuglini. The possibility of other related taxa (e.g. L. cachinnans) is not ruled out (Ash \& Atkins 2009).

C. h. hybrida as a Palaearctic winter visitor in Ethiopia and Oromia. African breeding $C$. h. delalandii might be in SW Ethiopia just outside Oromia, but no clear evidence (Ash \& Atkins 2009).

First record for Ethiopia on Lake Awasa in 2011 (Fisher et al. 2012). The lake is located just outside of Oromia, but on the way to this landlocked place, the bird crossed Oromia almost certainly. Not included in Oromia checklist.

SM Two subspecies in Ethiopia and Oromia. Within Oromia P. e. ellioti mainly in Rift Valley and Middle Awash area, P. e. olivascens in $\mathrm{S}$
(Ash \& Atkins 2009).

SM

SM

Two subspecies in Ethiopia and Oromia. Within Oromia

P. l. lichtensteinii in C and NE, P. l. sukensis in S (Ash \& Atkins 2009).

SM

Feral birds in towns and some villages. C. l. domestica

(cf. Ash \& Atkins 2009) not acknowledged by Clements et al. (2015).

$\mathrm{H}+$

All three subspecies said to be in Ethiopia and most likely in Oromia (Urban \& Brown 1986, Ash \& Atkins 2009). No further details, verification required.

Two subspecies in Ethiopia and Oromia. Within Oromia $S$, $r$ arabica in $\mathrm{C}$ and NE, $S$. r. roseogrisea most likely in W with one occupied 1 -degree-tetrad. Subspecific distribution in W Ethiopia not clearly understood (Ash \& Atkins 2009).

Two subspecies in Ethiopia and Oromia. Within Oromia $S$. d. decipiens widespread with greatest numbers through the Rift Valley. S. d. elegans in SE with two occupied 1-degree-tetrads. (Ash \& Atkins 2009). 


\begin{tabular}{|c|c|}
\hline $4981 / 329$ & $\begin{array}{l}\text { Streptopelia capicola electa } \\
\text { (Madarasz, 1913) }\end{array}$ \\
\hline $4982 / 320$ & $\begin{array}{l}\text { Streptopelia capicola somalica } \\
\text { (Erlanger, 1905) }\end{array}$ \\
\hline $4983 / 320$ & $\begin{array}{l}\text { Streptopelia capicola tropica } \\
\text { (Reichenow, 1902) }\end{array}$ \\
\hline $5446 / 329$ & $\begin{array}{l}\text { Treron calvus uellensis } \\
\text { (Reichenow, 1912) }\end{array}$ \\
\hline $5775 / 329$ & $\begin{array}{l}\text { Tauraco leucotis leucotis } \\
\text { (Rüppell, 1835) }\end{array}$ \\
\hline $5776 / 329$ & $\begin{array}{l}\text { Tauraco leucotis donaldsoni } \\
\text { (Sharpe, 1895) }\end{array}$ \\
\hline 777 / 337 & Tauraco ruspolii \\
\hline
\end{tabular}

(Salvadori, 1896)
$5790 / 338$

Corythaixoides personatus personatus

(Rüppell, 1842)

$5803 / 341$

$5804 / 341$

$5828 / 345$

$5829 / 34$

$5835 / 346$

$5836 / 346$

$5984 / 351$

$5985 / 351$

$6107 / 353$

$6108 / 353$

$6117 / 355$

$6238 / 356$

$6364 / 359$

$6365 / 359$

$6546 / 362$

$6609 / 367$

Clamator jacobinus pica

(Hemprich \& Ehrenberg, 1833)

Clamator jacobinus serratus

(Sparrman, 1786)

Cuculus clamosus gabonensis

Lafresnaye, 1853

Cuculus clamosus clamosus

Latham, 1802

Cuculus canorus canorus

Linnaeus, 1758

Cuculus canorus subtelephonus

Zarudny, 1914

Ceuthmochares aereus aereus

(Vieillot, 1817)

Ceuthmochares aereus australis

Sharpe, 1873

Centropus monachus fischeri

Reichenow, 1887

Centropus monachus monachus

Rüppell, 1837

Centropus superciliosus superciliosus

Hemprich \& Ehrenberg, 1829

Tyto alba affinis

(Blyth, 1862)

Otus scops scops

(Linnaeus, 1758)

Otus scops pulchellus

(Pallas, 1771)

Bubo capensis dillonii

Des Murs \& Prevost, 1846

Glaucidium perlatum licua

(Lichtenstein, 1842)
SM Three subspecies in Ethiopia and Oromia. Within Oromia S. c. electa in C (Rift Valley), S. c. somalica in E and S. c. tropica in NE and probably in far SW, based on specimen from the early $20^{\text {th }}$ century. Lines of distriSM bution in Ash \& Atkins' (2009) map provisional.

SM

$\mathrm{H}+\quad \mathrm{AH}$ Two subspecies in Ethiopia and Oromia. Within Oromia T. l. leucotis widespread. $T$. $l$. donaldsoni in E, nearly restricted to Oromia. In Bale Mts. the latter is found alongside with $T$. l. leucotis, supporting the view

x O, E, H AH for its specific separation (Ash \& Atkins 2009). Research needed.

$\mathrm{x} \quad \mathrm{O}, \mathrm{E}, \mathrm{H} \quad \mathrm{SM} \quad$ T. ruspoli is an endemic to Oromia with a very small range

(Borghesio \& Massa 2000). According to recent studies the species is still abundant where appropriate habitat occurs. However, the frequency decreases rapidly as the amount of agriculture in the landscape increases. Hybridizes with T. leucotis. In landscapes modified by human activities, Hybridizes with $T$. leucotis. In landscapes modified by human activities,
the two species of turacos might come in contact much more frequently, and chances of hybridization might become higher. Conservation efforts and further studies needed.

E, H SM C.p.personatus restricted to Rift valley of Ethiopia (Clements et al. 2015), core range in Oromia.

Two subspecies in Ethiopia and Oromia. C. j. pica and C.j. serratus are both intra-tropical migrants, with one population of $C . j$. pica breeding across Sub-Saharan Africa, and another from north-west India visiting eastern Africa. The relative status of the two populations in Ethiopia is unknown. C. $j$. serratus breeds in southern Africa, and then migrates north (Ash \& Atkins 2009).

Two valid subspecies in Ethiopia and Oromia. Within Oromia C. c. gabonensis in $\mathrm{W}$, and C. c. clamosus in $\mathrm{E}$. Birds in $\mathrm{S}$ referred to as C. c. jacksoni represent an intermediate or intergrade population, not a C. c. jacksoni represent an intermediate or intergrade populatid
valid subspecies (Ash \& Atkins 2009, del Hoyo et al. 1997).

Two subspecies in Ethiopia and Oromia, both are Palaearctic passage migrants (Ash \& Atkins 2009).

C. aereus is an elusive and seldom seen presumed resident in Ethiopia and Oromia. Within Oromia only two occupied 1-degree-tetrads (Rift Valley). Birds generally attributed to C. a. australis, but they are perhaps more likely C. a. aereus, or possibly even a new subspecies

(Ash \& Atkins 2009).

Two subspecies in Ethiopia and most likely in Oromia. Within Oromia C. m. monachus in $\mathrm{C}, \mathrm{S}$ and $\mathrm{W}$ almost to Sudan border, where it meets $C$. m. fischeri (van den Elzen \& König 1983). No further details on distribuSM tion in Ethiopia (Ash \& Atkins 2009). Clarification needed.

SM

Scattered records from Ethiopia and Oromia. All collected birds have been identified as $O$. s. scops, except for one record of $O$. s. pulchellus from Moulou (1-degree-tetrad in NE Oromia). Both subspecies Palaearctic winter visitors (Ash \& Atkins 2009).

E, $\mathrm{H} \quad \mathrm{AH}$ Core range of B. c. dilloni in Oromia, with few, mostly uncertain records from other parts of Ethiopia, and only one recent record in Eritrea (Ash \& Atkins 2009). 


\section{$6734 / 368$}

Athene noctua somaliensi

Reichenow, 1905

$6825 / 366$

$6836 / 369$

(Heuglin, 1863)

Asio abyssinicus abyssinicu

(Guerin-Meneville, 1843)

$7228 / 372$

Caprimulgus europaeus europaeus

Linnaeus, 1758

$7231 / 372$

Caprimulgus europaeus unwin

Hume, 1871

$7246 / 375$

Caprimulgus nubicus torridus

Lort Phillips, 1898

$7292 /$

Safford, Ash, Duckworth, Telfer and Zewdie,

1995

$7319 / 383$

Caprimulgus climacurus nigricans

(Salvadori, 1868)

$7586 / 388$

Apus melba melba

(Linnaeus, 1758)

$7589 / 388$

Apus melba africanus

(Temminck, 1815)

$7603 / 390$

Apus apus apus

(Linnaeus, 1758)

$7604 / 390$

apus apus pekinensi

(Swinhoe, 1870)

$7620 /$

Apus barbatus roehli

Reichenow, 1906

$7644 / 392$

Apus affinis aerobates

Brooke, 1969

$7646 / 392$

Apus affinis affinis

(J. E. Gray, 1830)

$7682 / 387$

Cypsiurus parvus parvus

(Lichtenstein, 1823)

$7684 / 387$

Cypsiurus parvus myochrous

(Reichenow, 1886)

$7685 / 387$

Cypsiurus parvus laemostigma

(Reichenow, 1905)

$8655 / 395$

Colius striatus mombassicus

Someren, 1919

$8662 / 395$

Colius striatus leucotis

Rüppell, 1839

$8663 / 395$

Zedlitz, 1910

$8673 / 396$

(Neumann, 1900)

$8674 / 396$

Urocolius macrourus abyssinicus

Schifter, 1975

$8675 / 396$
$\mathrm{E}, \mathrm{H}+$

E, $\mathrm{H}$ AH A. a. abyssinicus restricted to Ethiopia, core range in Oromia

A. m. africanus presumed resident and intra-tropical migrant. A. m. melba assumed Palaearctic visitor, but no evidence so far (Ash \& Atkins 2009)

One, maybe two subspecies in Ethiopia and Oromia. Passage migrant, some possibly overwintering. A. a. pekinensis confirmed for Ethiopia from a single specimen (Addis Ababa). One A. a. apus specimen collected in Eritrea, but this subspecies may occur also in Ethiopia and Oromia (Ash \& Atkins 2009)

Recorded for the first time in 2014 between Goba and Sannetti plateau (Andrews 2015). Subspecies not identified, according to Clements et al. (2015) roehli most likely.

A. a. aerobates in Ethiopia and Oromia. A. a. affinis could occur in the region close to the southern border, but not confirmed so far (Ash \& Atkins 2009).

In Ethiopia two, possibly all three described subspecies are involved. In $\mathrm{W}$ and SW Oromia probably C. p. myochorus, but this subspecies not confirmed with a specimen. In SE Oromia $C$. p. laemostigma (three occupied 1-degree-tetrads). C. p. parvus could occur in NW Oromia, but no record so far The subspecies of the birds in the northern Rift is unknown (Ash \& Atkins 2009). Clarification needed.

C. s. leucotis widespread in Ethiopia, incl. Oromia. C. s. mombassicus could be expected in SE Oromia, and C. s. hilgerti in NE Oromia, but no records so far (Ash \& Atkins 2009).

SM Four subspecies in Ethiopia, two of them in Oromia. U. m. abyssinicus in C, E, S Oromia, and U. m. griseogularis in W Oromia. U. m. pulcher could be expected in SE Oromia, but no record so far

SM (Ash \& Atkins 2009). 


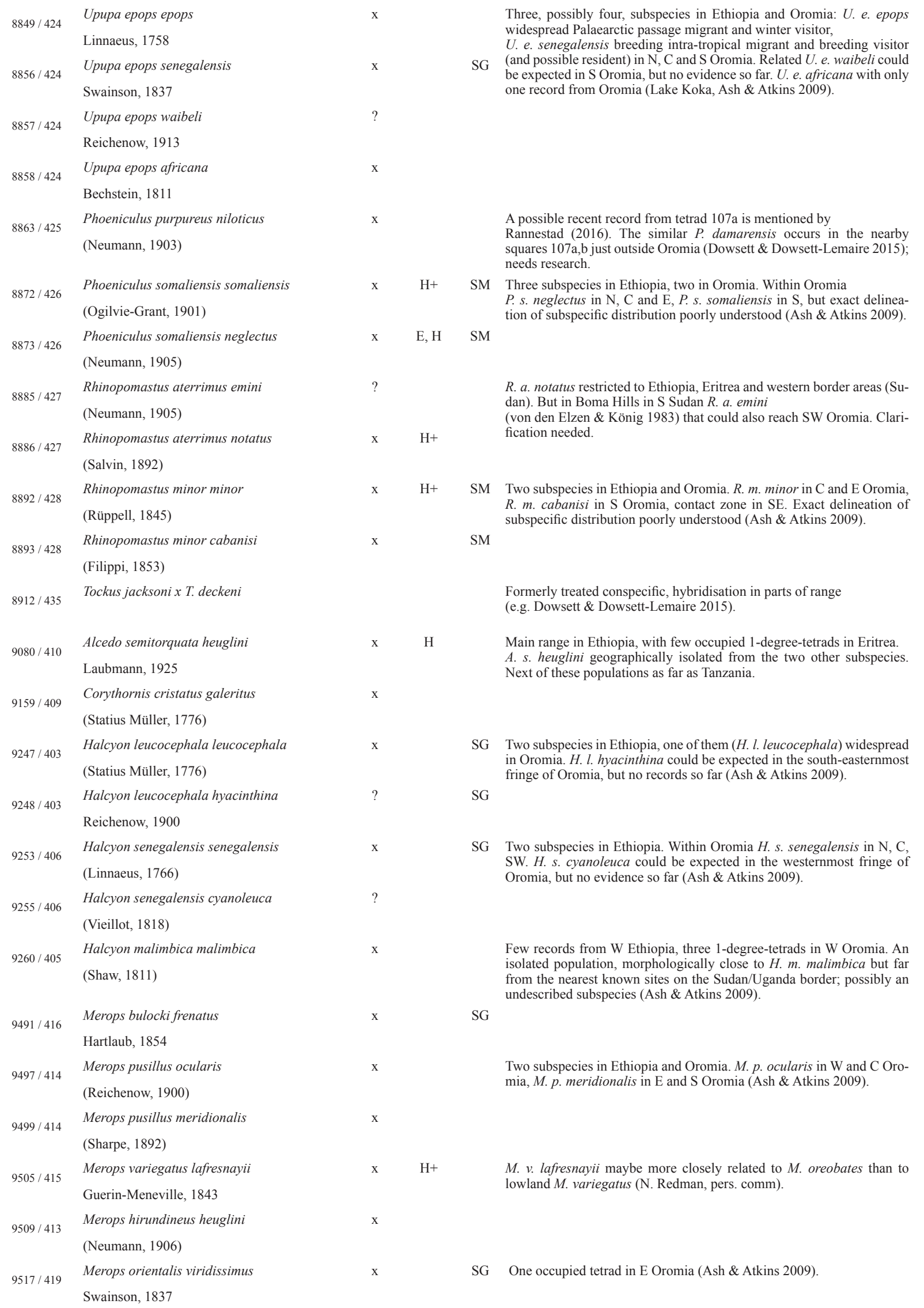




\begin{tabular}{|c|c|c|c|c|c|}
\hline $9553 / 399$ & $\begin{array}{l}\text { Coracias caudatus lorti } \\
\text { Shelley, } 1885\end{array}$ & $\mathrm{x}$ & $\mathrm{H}^{+}$ & SM & \\
\hline $9557 / 398$ & $\begin{array}{l}\text { Coracias naevius naevius } \\
\text { Daudin, } 1800\end{array}$ & $\mathrm{x}$ & & SG & \\
\hline $9568 / 402$ & $\begin{array}{l}\text { Eurystomus glaucurus afer } \\
\text { (Latham, 1790) }\end{array}$ & $?$ & & & $\begin{array}{l}\text { According to Ash \& Atkins (2009) E. g. afer in W Ethiopia. But accord- } \\
\text { ing to del Hoyo et al. (2001) and Clements et al. (2015) E. g. aethiopicus. } \\
\text { Clarification needed. }\end{array}$ \\
\hline $9569 / 402$ & $\begin{array}{l}\text { Eurystomus glaucurus aethiopicus } \\
\text { Neumann, } 1905\end{array}$ & $?$ & & SG & \\
\hline 9749 / 446 & $\begin{array}{l}\text { Trachyphonus erythrocephalus shelleyi } \\
\text { Hartlaub, } 1886\end{array}$ & $\mathrm{x}$ & $\mathrm{H}$ & SM & $\begin{array}{l}\text { Two subspecies in Ethiopia. Within Oromia T. e. shelleyi in NE, T. e. } \\
\text { versicolor in S, but range of each unclear and much intergradation where } \\
\text { they meet (Ash \& Atkins 2009). Two other proposed subspecies from }\end{array}$ \\
\hline 9750 / 446 & $\begin{array}{l}\text { Trachyphonus erythrocephalus versicolor } \\
\text { Hartlaub, } 1882\end{array}$ & $\mathrm{x}$ & & SM & $\begin{array}{l}\text { Ethiopia (T. e gallarum, T. e. jacksoni) probably represent intergrades } \\
\text { (del Hoyo et al. 2002) and are not acknowledged. }\end{array}$ \\
\hline 9753 / 447 & $\begin{array}{l}\text { Trachyphonus margaritatus margaritatus } \\
\text { (Cretzschmar, 1828) }\end{array}$ & $\mathrm{x}$ & & & $\begin{array}{l}\text { Two subspecies in Ethiopia. Within Oromia T. m. margaritatus in C and } \\
\text { NE, T. m. somalicus even further to the NE, with some overlapping in the } \\
\text { Harar area (Ash \& Atkins 2009). }\end{array}$ \\
\hline 9754 / 447 & $\begin{array}{l}\text { Trachyphonus margaritatus somalicus } \\
\text { Zedlitz, } 1910\end{array}$ & $\mathrm{x}$ & $\mathrm{H}$ & SM & \\
\hline $9757 / 448$ & $\begin{array}{l}\text { Trachyphonus darnaudii darnaudii } \\
\text { (Prevost \& Des Murs, 1847) }\end{array}$ & $\mathrm{x}$ & & SM & $\begin{array}{l}\text { Two subspecies in Ethiopia. Within Oromia } T . d \text {. darnaudi common in } \\
\mathrm{S} \text { and } T . d \text {. boehmi patchily distributed in the E. Possible overlap and } \\
\text { contact zones of the two ranges not known (Ash \& Atkins 2009). }\end{array}$ \\
\hline $9758 / 448$ & $\begin{array}{l}\text { Trachyphonus darnaudii boehmi } \\
\text { Fischer \& Reichenow, } 1884\end{array}$ & $\mathrm{x}$ & & SM & \\
\hline $9816 / 438$ & $\begin{array}{l}\text { Pogoniulus pusillus uropygialis } \\
\text { (Heuglin, 1862) }\end{array}$ & $\mathrm{x}$ & $\mathrm{H}$ & SM & $\begin{array}{l}\text { Two subspecies in Ethiopia. Within Oromia } P . p \text {. uropygialis in the far } \\
\mathrm{N} \text { and NE, P. p. affinis in C and S. Dividing line shown on the map in } \\
\text { Ash \& Atkins (2009) only approximate. }\end{array}$ \\
\hline $9817 / 438$ & $\begin{array}{l}\text { Pogoniulus pusillus affinis } \\
\text { (Reichenow, 1879) }\end{array}$ & $\mathrm{x}$ & & SM & \\
\hline $9820 / 439$ & $\begin{array}{l}\text { Pogoniulus chrysoconus chrysoconus } \\
\text { (Temminck, 1832) }\end{array}$ & $?$ & & SG & $\begin{array}{l}\text { Two subspecies in Ethiopia. Within Oromia only } P . c \text {. xanthostictus } \\
\text { which is mainly found in the highlands of Oromia west and east of the } \\
\text { Rift Valley. } P . \text { c. chrysoconus in Tigre and Amhara Regional State, with }\end{array}$ \\
\hline $9821 / 439$ & $\begin{array}{l}\text { Pogoniulus chrysoconus xanthostictus } \\
\text { (Blundell \& Lovat, 1899) }\end{array}$ & $\mathrm{x}$ & E, $\mathrm{H}$ & & $\begin{array}{l}\text { southernmost records just outside Oromia (Ash \& Atkins 2009). Occur- } \\
\text { rence in Oromia conceivable. }\end{array}$ \\
\hline $9832 / 440$ & $\begin{array}{l}\text { Tricholaema diademata diademata } \\
\text { (Heuglin, 1861) }\end{array}$ & $\mathrm{x}$ & & SM & \\
\hline $9843 / 441$ & $\begin{array}{l}\text { Tricholaema melanocephala melanocephala } \\
\text { (Cretzschmar, 1829) }\end{array}$ & $\mathrm{x}$ & $\mathrm{H}$ & SM & $\begin{array}{l}\text { Three subspecies in Ethiopia. Within Oromia T. m. melanocephala in } \mathrm{N} \\
\text { (to } 8^{\circ} \mathrm{N} \text { in Rift Valley), T. m. stigmatothorax in S and SE, T. m. blandi far } \\
\text { E with only one occupied 1-degree-tetrad (Ash \& Atkins 2009). }\end{array}$ \\
\hline 9844 / 441 & $\begin{array}{l}\text { Tricholaema melanocephala stigmatothorax } \\
\text { Cabanis, } 1878\end{array}$ & $\mathrm{x}$ & & SM & \\
\hline 9845 / 441 & $\begin{array}{l}\text { Tricholaema melanocephala blandi } \\
\text { Lort Phillips, } 1897\end{array}$ & $\mathrm{x}$ & $\mathrm{H}$ & SM & \\
\hline 9849 / 442 & $\begin{array}{l}\text { Lybius undatus undatus } \\
\text { (Rüppell, 1837) }\end{array}$ & $\mathrm{x}$ & E, $\mathrm{H}$ & $\mathrm{AH}$ & $\begin{array}{l}\text { Four subspecies in Ethiopia, thereof three in Oromia. Within Oromia } \\
\text { L. u. undunatus in N, L. u. leucogenys in C, W and SW, and } \\
\text { L. u. salvadori in NE (Ash \& Atkins 2009). The latter regarded as squa- }\end{array}$ \\
\hline $9850 / 442$ & $\begin{array}{l}\text { Lybius undatus leucogenys } \\
\text { (Blundell \& Lovat, 1899) }\end{array}$ & $\mathrm{x}$ & & $\mathrm{AH}$ & $\begin{array}{l}\text { matus by Ash \& Atkins (2009) without further explanation. However, } L \text {. } \\
\text { u. squamatus considered untenable by } \\
\text { del Hoyo et al. (2002). }\end{array}$ \\
\hline $9850 / 442$ & $\begin{array}{l}\text { Lybius undatus leucogenys } \\
\text { (Blundell \& Lovat, 1899) }\end{array}$ & $\mathrm{x}$ & & $\mathrm{AH}$ & \\
\hline $9851 / 442$ & $\begin{array}{l}\text { Lybius undatus salvadorii } \\
\text { Neumann, } 1903\end{array}$ & $\mathrm{x}$ & & $\mathrm{AH}$ & \\
\hline $9881 / 445$ & $\begin{array}{l}\text { Lybius bidentatus aequatorialis } \\
\text { (Shelley, 1889) }\end{array}$ & $\mathrm{x}$ & & & \\
\hline $10143 / 449$ & $\begin{array}{l}\text { Prodotiscus zambesiae ellenbecki } \\
\text { Erlanger, } 1901\end{array}$ & $\mathrm{x}$ & & & \\
\hline
\end{tabular}




\begin{tabular}{|c|c|c|c|c|c|}
\hline $10168 / 451$ & $\begin{array}{l}\text { Indicator minor diademata } \\
\text { Rüppell, } 1837\end{array}$ & $\mathrm{x}$ & & & $\begin{array}{l}\text { Two subspecies in Ethiopia and Oromia. } I . m \text {. diademata widespread, } I \text {. } \\
\text { m. teitensis in the southernmost areas along the border to Kenya and So- } \\
\text { malia. Few records from Oromia around Moyale (Ash \& Atkins 2009). }\end{array}$ \\
\hline $10170 / 451$ & $\begin{array}{l}\text { Indicator minor teitensis } \\
\text { Neumann, } 1900\end{array}$ & $\mathrm{x}$ & & & \\
\hline $10192 / 455$ & $\begin{array}{l}\text { Jynx ruficollis aequatorialis } \\
\text { Rüppell, } 1842\end{array}$ & $\mathrm{x}$ & E, $\mathrm{H}$ & $\mathrm{AH}$ & $\begin{array}{l}\text { Endemic to Ethiopia. Mainly distributed in the highlands of Oromia over } \\
\text { rather restricted areas (Ash \& Atkins 2009). }\end{array}$ \\
\hline $10394 / 457$ & $\begin{array}{l}\text { Campethera cailliautii nyansae } \\
\text { (Neumann, 1900) }\end{array}$ & $\mathrm{x}$ & & & $\begin{array}{l}\text { Rare in SW Ethiopia. Within Oromia only two occupied 1-degree-tet- } \\
\text { rads. }\end{array}$ \\
\hline $10421 / 459$ & $\begin{array}{l}\text { Dendropicos fuscescens lepidus } \\
\text { (Cabanis \& Heine, 1863) }\end{array}$ & $\mathrm{x}$ & & $\mathrm{AH}$ & $\begin{array}{l}\text { Three subspecies in Ethiopia. Within Oromia } D . f \text {. hemprichii in } \mathrm{C}, \mathrm{N} \\
\text { and } \mathrm{NE}, D \text {. } f \text {. lepidus in } \mathrm{W} \text {, and } D . f . \text { massaicus in S. Interbreed where } \\
\text { they meet and distribution and relative status poorly understood. (Ash \& }\end{array}$ \\
\hline $10422 / 459$ & $\begin{array}{l}\text { Dendropicos fuscescens massaicus } \\
\text { Neumann, } 1900\end{array}$ & $\mathrm{x}$ & & SM & Atkins 2009). Research needed. \\
\hline $10423 / 459$ & $\begin{array}{l}\text { Dendropicos fuscescens hemprichii } \\
\text { (Ehrenberg, 1833) }\end{array}$ & $\mathrm{x}$ & $\mathrm{H}+$ & SM & \\
\hline $10431 / 460$ & $\begin{array}{l}\text { Dendropicos namaquus schoensis } \\
\text { (Rüppell, 1842) }\end{array}$ & $\mathrm{x}$ & $\mathrm{H}+$ & SM & \\
\hline $10440 / 461$ & $\begin{array}{l}\text { Dendropicos goertae abessinicus } \\
\text { (Reichenow, 1900) }\end{array}$ & $\mathrm{x}$ & & SG & \\
\hline $10446 / 461$ & $\begin{array}{l}\text { Dendropicos spodocephalus spodocephalus } \\
\text { (Bonaparte, 1850) }\end{array}$ & $\mathrm{x}$ & $\mathrm{E}, \mathrm{H}+$ & & \\
\hline $10454 / 462$ & $\begin{array}{l}\text { Dendropicos obsoletus ingens } \\
\text { (Hartert, 1900) }\end{array}$ & $\mathrm{x}$ & & & \\
\hline $11081 / 96$ & $\begin{array}{l}\text { Polihierax semitorquatus } \\
\text { (A. Smith, 1836) }\end{array}$ & $\mathrm{x}$ & & & $\begin{array}{l}\text { P. semitorquatus has two separated and very distinct ranges in SW Africa } \\
\text { and NE Africa, respectively. Birds from NE Africa sometimes separated } \\
\text { as P. s. castanonotus, but poorly differentiated. }\end{array}$ \\
\hline $11098 / 98$ & $\begin{array}{l}\text { Falco tinnunculus tinnunculus } \\
\text { Linnaeus, } 1758\end{array}$ & $\mathrm{x}$ & & & $\begin{array}{l}\text { F. } t \text {. rufescens is an uncommon breeding resident in Ethiopia. There are } \\
\text { only twelve records, all from May to September. F. } t \text {. archeri occurs to an } \\
\text { unknown extent along the border to Somalia. Most birds seen in Ethiopia }\end{array}$ \\
\hline 11099 / 98 & $\begin{array}{l}\text { Falco tinnunculus archerii } \\
\text { Hartert \& Neumann, } 1932\end{array}$ & $\mathrm{x}$ & & & $\begin{array}{l}\text { are Palaearctic overwintering migrant } F . \text { t. tinnunculus } \\
\text { (Ash \& Atkins 2009). }\end{array}$ \\
\hline $11109 / 98$ & $\begin{array}{l}\text { Falco tinnunculus rufescens } \\
\text { Swainson, } 1837\end{array}$ & $\mathrm{x}$ & & & \\
\hline $11123 / 99$ & $\begin{array}{l}\text { Falco rupicoloides fieldi } \\
\text { (Elliot, 1897) }\end{array}$ & $\mathrm{x}$ & $\mathrm{H}$ & SM & $\begin{array}{l}\text { Only a few scattered records from Ethiopia. Within Oromia } F . r \text { fieldi in } \\
\text { NE, only one occupied 1-degree-tetrad. F. r. arthuri in SW } \\
\text { (Ash \& Atkins 2009). Nominate rupicoloides in SW Africa, geographi- }\end{array}$ \\
\hline $11124 / 99$ & $\begin{array}{l}\text { Falco rupicoloides arthuri } \\
\text { (Gurney, 1884) }\end{array}$ & $\mathrm{x}$ & & SM & cally isolated from NE African populations. \\
\hline $11153 / 102$ & $\begin{array}{l}\text { Falco chicquera ruficollis } \\
\text { Swainson, } 1837\end{array}$ & $\mathrm{x}$ & & & \\
\hline $11155 / 103$ & $\begin{array}{l}\text { Falco vespertinus } \\
\text { Linnaeus, } 1766\end{array}$ & $?$ & & & $\begin{array}{l}\text { Few records, but not confirmed (Ash \& Atkins 2009). Not included in } \\
\text { Oromia checklist. }\end{array}$ \\
\hline $11198 / 109$ & $\begin{array}{l}\text { Falco biarmicus abyssinicus } \\
\text { Neumann, } 1904\end{array}$ & $\mathrm{x}$ & & & \\
\hline $11209 / 111$ & $\begin{array}{l}\text { Falco peregrinus calidus } \\
\text { Latham, } 1790\end{array}$ & $\mathrm{x}$ & & & $\begin{array}{l}\text { Two subspecies in Ethiopia and Oromia. Uncommon } F . p \text {. minor is a } \\
\text { breeding resident, } F \text {. p. calidus a Palaearctic winter visitor and passage } \\
\text { migrant (Ash \& Atkins 2009). }\end{array}$ \\
\hline $11219 / 111$ & $\begin{array}{l}\text { Falco peregrinus minor } \\
\text { Bonaparte, } 1850\end{array}$ & $\mathrm{x}$ & & & \\
\hline $11790 / 331$ & $\begin{array}{l}\text { Agapornis pullarius pullarius } \\
\text { (Linnaeus, 1758) }\end{array}$ & $?$ & & & $\begin{array}{l}\text { Both A. p. ugandae and A. p. pullaris are recorded from SE Ethiopia, but } \\
\text { confirmation is desirable for A. p. pullaris (Ash \& Atkins 2009). }\end{array}$ \\
\hline $11791 / 331$ & $\begin{array}{l}\text { Agapornis pullarius ugandae } \\
\text { Neumann, } 1908\end{array}$ & $\mathrm{x}$ & & & \\
\hline
\end{tabular}




\begin{tabular}{|c|c|c|c|c|c|}
\hline $11818 / 333$ & $\begin{array}{l}\text { Poicephalus meyeri meyeri } \\
\text { (Cretzschmar, 1827) }\end{array}$ & $\mathrm{x}$ & & & $\begin{array}{l}\text { One, maybe two subspecies in Ethiopia and Oromia. Within Oromia } \\
\text { P. m. meyeri in NW with one occupied 1-degree-tetrad. P. m. saturatus } \\
\text { with only one uncertain record in S (Ash \& Atkins 2009). }\end{array}$ \\
\hline $11819 / 333$ & $\begin{array}{l}\text { Poicephalus meyeri saturatus } \\
\text { (Sharpe, 1901) }\end{array}$ & ? & & & \\
\hline $11830 / 334$ & $\begin{array}{l}\text { Poicephalus rufiventris pallidus } \\
\text { Someren, } 1922\end{array}$ & ? & $\mathrm{H}$ & SM & \multirow{2}{*}{$\begin{array}{l}\text { One, maybe two subspecies in Ethiopia and Oromia. Within Oromia } \\
P . r \text { rufiventris in E and S. P. } r \text {. pallidus could be expected on the east- } \\
\text { ernmost fringes, current records from tetrads just outside Oromia (Ash \& } \\
\text { Atkins 2009) }\end{array}$} \\
\hline $11831 / 334$ & $\begin{array}{l}\text { Poicephalus rufiventris rufiventris } \\
\text { (Rüppell, 1845) }\end{array}$ & $\mathrm{x}$ & & SM & \\
\hline $12383 / 463$ & $\begin{array}{l}\text { Pitta angolensis longipennis } \\
\text { Reichenow, } 1901\end{array}$ & $\mathrm{x}$ & & & \\
\hline $17405 / 467$ & $\begin{array}{l}\text { Platysteira cyanea aethiopica } \\
\text { Neumann, } 1905\end{array}$ & $\mathrm{x}$ & $\mathrm{E}, \mathrm{H}+$ & & Restricted to Ethiopia, core range in Oromia (Ash \& Atkins 2009). \\
\hline $17455 / 464$ & $\begin{array}{l}\text { Batis orientalis bella } \\
\text { (Elliot, } 18979 \\
\text { Batis orientalis orientalis } \\
\text { (Heuglin, 1871) }\end{array}$ & $\mathrm{x}$ & $\mathrm{H}^{+}$ & SM & $\begin{array}{l}\text { Three subspecies in Ethiopia and Oromia. In the distribution map in } \\
\text { Ash \& Atkins (2009) B. o. bella is lumped with B. o. orientalis. The lat- } \\
\text { ter is widespread in Oromia, B. o. bella in S Oromia. Area of division is } \\
\text { unclear. B. o. chadensis only in westernmost parts (Ash \& Atkins 2009). } \\
\text { Dowsett \& Dowsett-Lemaire (2015) state that the broad geographic } \\
\text { overlap with B. orientalis might be erroneous. }\end{array}$ \\
\hline $17456 / 464$ & $\begin{array}{l}\text { Batis orientalis chadensis } \\
\text { Alexander, } 1908\end{array}$ & $\mathrm{x}$ & & & \\
\hline $17459 / 465$ & $\begin{array}{l}\text { Batis minor erlangeri } \\
\text { Neumann, } 1907\end{array}$ & $\mathrm{x}$ & & & $\begin{array}{l}\text { Sometimes treated as a separate species Batis erlangeri (del Hoyo et al. } \\
\text { 2006). Dowsett \& Dowsett-Lemaire }(2015) \text { state that the broad ge- } \\
\text { graphic overlap with } B \text {. orientalis might be erroneous. }\end{array}$ \\
\hline $17473 / 468$ & $\begin{array}{l}\text { Prionops plumatus concinnatus } \\
\text { Sundevall, } 1850\end{array}$ & $\mathrm{x}$ & & SG & $\begin{array}{l}\text { Three subspecies in Ethiopia. Within Oromia } P . p \text {. concinnatus in W, } P \text {. } \\
\text { p. cristatus in C and SW, and } P . \text { p. vinaceigularis in E and SE } \\
\text { (Ash \& Atkins 2009). }\end{array}$ \\
\hline $17474 / 468$ & $\begin{array}{l}\text { Prionops plumatus cristatus } \\
\text { Rüppell, } 1836\end{array}$ & $\mathrm{x}$ & & SM & \\
\hline $17475 / 468$ & $\begin{array}{l}\text { Prionops plumatus vinaceigularis } \\
\text { Richmond, } 1897\end{array}$ & $\mathrm{x}$ & & SM & \\
\hline $17574 / 481$ & $\begin{array}{l}\text { Nilaus afer minor } \\
\text { Sharpe, } 1895\end{array}$ & $\mathrm{x}$ & & SM & $\begin{array}{l}N . \text { a. minor throughout Oromia. Proposed subspecies } N . \text { a. hilgerti syn- } \\
\text { onymised with } N \text {. a. minor (del Hoyo et al. } 2009 \text { ). Map in } \\
\text { Ash \& Atkins (2009) shows } N \text {. a. hilgerti and } N \text {. a. minor separately. }\end{array}$ \\
\hline $17585 / 475$ & $\begin{array}{l}\text { Dryoscopus gambensis erythreae } \\
\text { O. Neumann, } 1899\end{array}$ & $\mathrm{x}$ & $\mathrm{H}^{+}$ & & \\
\hline $17615 / 474$ & $\begin{array}{l}\text { Tchagra senegalus habessinicus } \\
\text { (Ehrenberg, 1833) }\end{array}$ & $\mathrm{x}$ & & & \\
\hline $17638 / 478$ & $\begin{array}{l}\text { Laniarius ruficeps rufinuchalis } \\
\text { (Sharpe, 1895) }\end{array}$ & $\mathrm{x}$ & $\mathrm{H}^{+}$ & SM & \\
\hline $17673 / 477$ & $\begin{array}{l}\text { Laniarius funebris funebris } \\
\text { (Hartlaub, 1863) }\end{array}$ & $\mathrm{x}$ & & SM & $\begin{array}{l}\text { Demarcation line between } L . f . \text { funebris and } L . f . \text { deneger in SE Ethiopia } \\
\text { unclear, subspecies not differentiated in Ash \& Atkins (2009). } \\
\text { L.f. deneger could reach southern and easternmost Oromia. }\end{array}$ \\
\hline $17674 / 477$ & $\begin{array}{l}\text { Laniarius funebris degener } \\
\text { Hilgert, } 1912\end{array}$ & $?$ & & SM & \\
\hline $17688 / 471$ & $\begin{array}{l}\text { Rhodophoneus cruentus hilgerti } \\
\text { (Neumann, 1903) }\end{array}$ & $\mathrm{x}$ & $\mathrm{H}+$ & SM & \\
\hline $17701 / 470$ & $\begin{array}{l}\text { Telophorus sulfureopectus similis } \\
\text { (A. Smith, 1836) }\end{array}$ & $\mathrm{x}$ & & & \\
\hline $17731 / 469$ & $\begin{array}{l}\text { Malaconotus blanchoti catharoxanthus } \\
\text { Neumann, } 1899\end{array}$ & $\mathrm{x}$ & & SG & $\begin{array}{l}\text { Two subspecies in Ethiopia. Within Oromia } M . b . \text { catharoxanthus rare } \\
\text { in W and NW (two occupied 1-degree-tetrads), M. b. approximans else- } \\
\text { where (Ash \& Atkins 2009). }\end{array}$ \\
\hline $17732 / 469$ & $\begin{array}{l}\text { Malaconotus blanchoti approximans } \\
\text { (Cabanis, 1869) }\end{array}$ & $\mathrm{x}$ & & SM & \\
\hline $17944 / 482$ & $\begin{array}{l}\text { Coracina caesia pura } \\
\text { (Sharpe, 1891) }\end{array}$ & $\mathrm{x}$ & & $\mathrm{AH}$ & \\
\hline $18513 / 488$ & $\begin{array}{l}\text { Lanius phoenicuroides } \\
\text { (Schalow, 1875) }\end{array}$ & $\mathrm{x}$ & & & $\begin{array}{l}\text { Formerly considered a subspecies of Lanius isabellinus. Palaearctic pas- } \\
\text { sage migrant. }\end{array}$ \\
\hline
\end{tabular}




\begin{tabular}{|c|c|c|c|c|c|}
\hline $18515 / 488$ & Lanius isabellinus isabellinus & \multicolumn{3}{|l|}{$\mathrm{x}$} & \multirow[t]{2}{*}{ Palaearctic passage migrant. } \\
\hline & Hemprich \& Ehrenberg, 1833 & & & & \\
\hline $18586 / 490$ & $\begin{array}{l}\text { Lanius meridionalis aucheri } \\
\text { Bonaparte, } 1853\end{array}$ & $\mathrm{x}$ & & & $\begin{array}{l}\text { Two subspecies in Ethiopia. Within Oromia } L . m \text {. aucheri breeding resi- } \\
\text { dent in C and NE. L. m. pallidirostris an uncommon winter visitor, in the } \\
\text { south as far as } 5^{\circ} \mathrm{N} \text { (Ash \& Atkins 2009). }\end{array}$ \\
\hline $18592 / 490$ & $\begin{array}{l}\text { Lanius meridionalis pallidirostris } \\
\text { Cassin, } 1852\end{array}$ & $\mathrm{x}$ & & & \\
\hline $18598 / 491$ & $\begin{array}{l}\text { Lanius excubitoroides excubitoroides } \\
\text { Prévost \& Des Murs, } 1847\end{array}$ & $\mathrm{x}$ & & SG & $\begin{array}{l}\text { Two subspecies in Ethiopia and Oromia. L. e. intercedens in central- } \\
\text { southern areas and presumably L. e. excubitoroides elsewhere. Distribu- } \\
\text { tion boundaries between them not very clear }\end{array}$ \\
\hline $18599 / 491$ & $\begin{array}{l}\text { Lanius excubitoroides intercedens } \\
\text { Neumann, } 1905\end{array}$ & $\mathrm{x}$ & & & (Ash \& Atkins 2009). \\
\hline $18607 / 494$ & $\begin{array}{l}\text { Lanius humeralis humeralis } \\
\text { Stanley, } 1814\end{array}$ & $\mathrm{x}$ & & & In Ash \& Atkins (2009) referred to as Lanius collaris humeralis. \\
\hline $18626 / 495$ & $\begin{array}{l}\text { Lanius senator niloticus } \\
\text { (Bonaparte, 1853) }\end{array}$ & $\mathrm{x}$ & & & \\
\hline $18637 / 486$ & $\begin{array}{l}\text { Eurocephalus rueppelli } \\
\text { Bonaparte, } 1853\end{array}$ & $\mathrm{x}$ & & & $\begin{array}{l}\text { Monotypic species according to Clements et al. (2015). In } \\
\text { del Hoyo et al. (2008) two subspecies recognized: E. r. rueppelli in W } \\
\text { and part of S Ethiopia, and somewhat larger E. r. erlangeri in C and SE } \\
\text { Ethiopia. Map in Ash \& Atkins (2009) without differentiation of subspe- } \\
\text { cies. }\end{array}$ \\
\hline $19007 / 499$ & $\begin{array}{l}\text { Oriolus monacha monacha } \\
\text { (J. F. Gmelin, 1789) }\end{array}$ & $\mathrm{x}$ & $\mathrm{H}$ & $\mathrm{AH}$ & $\begin{array}{l}\text { Two subspecies in Ethiopia and Oromia: } O . \text { m. monacha north of } 9^{\circ} 30^{\prime} \mathrm{N} \\
\text { and } O . \text { m. meneliki south of this (Ash \& Atkins 2009). Precise informa- } \\
\text { tion difficult to assess, most notably because } O \text {. monacha meneliki easily }\end{array}$ \\
\hline $19008 / 499$ & $\begin{array}{l}\text { Oriolus monacha meneliki } \\
\text { Blundell \& Lovat, } 1899\end{array}$ & $\mathrm{x}$ & E, $\mathrm{H}$ & $\mathrm{AH}$ & $\begin{array}{l}\text { confused with } O \text {. larvatus rolletti in the field. Subspecies intergrade in } \mathrm{C} \\
\text { Ethiopia (del Hoyo et al. 2008). }\end{array}$ \\
\hline $19014 / 500$ & $\begin{array}{l}\text { Oriolus larvatus rolleti } \\
\text { Salvadori, } 1864\end{array}$ & $\mathrm{x}$ & & & $\begin{array}{l}\text { O. larvatus rolletti easily confused with } O \text {. monacha meneliki in the field } \\
\text { (cf. annotations on } O \text {. monacha sspp.). }\end{array}$ \\
\hline $19056 / 501$ & $\begin{array}{l}\text { Dicrurus adsimilis divaricatus } \\
\text { (Lichtenstein, 1823) }\end{array}$ & $\mathrm{x}$ & & & \\
\hline $19477 / 502$ & $\begin{array}{l}\text { Terpsiphone viridis ferreti } \\
\text { (Guérin-Méneville, 1843) }\end{array}$ & $\mathrm{x}$ & & & \\
\hline $20014 / 503$ & $\begin{array}{l}\text { Zavattariornis stresemanni } \\
\text { Moltoni, } 1938\end{array}$ & $\mathrm{x}$ & $\mathrm{O}, \mathrm{E}, \mathrm{H}$ & SM & $\begin{array}{l}\text { Z. stresemanni is restricted to the Borana zone in SE Oromia, with a total } \\
\text { range of } 6,000 \mathrm{~km}^{2} \text {. Range is a consequence of high reliance on modified } \\
\text { habitats within narrow climatic limits. Climate change may therefore } \\
\text { pose a serious threat to the species long-term survival } \\
\text { (Donald et al. } 2012 \text { ). For studies on distribution, habitat and biology see } \\
\text { Borghesio \& Giannetti (2005), Gedeon (2006), } \\
\text { Ross et al. (2009), Mellanby et al. (2008), Töpfer \& Gedeon (2012) and } \\
\text { Bladon } \text { et al. (2016). }\end{array}$ \\
\hline $20042 / 504$ & $\begin{array}{l}\text { Pyrrhocorax pyrrhocorax baileyi } \\
\text { Rand \& Vaurie, } 1955\end{array}$ & $\mathrm{x}$ & E, $\mathrm{H}$ & $\mathrm{AH}$ & $\begin{array}{l}\text { Geographically very isolated subspecies. In Sub-Saharan Africa only in } \\
\text { Simien and nearby highlands (N and NE Amhara) and in Bale Mts. (Oro- } \\
\text { mia). The two populations are separated by } 800 \mathrm{~km} \text { and may be subspe- } \\
\text { cifically distinct, but no Bale specimen exists for comparison } \\
\text { (Ash \& Atkins 2009). Next populations of P. pyrrhocorax as far as Israel } \\
\text { and Iran. }\end{array}$ \\
\hline $20087 / 507$ & $\begin{array}{l}\text { Corvus capensis kordofanensis } \\
\text { Laubmann, } 1919\end{array}$ & $\mathrm{x}$ & & & \\
\hline $20151 / 510$ & $\begin{array}{l}\text { Corvus edithae } \\
\text { E. L. Phillips, } 1895\end{array}$ & $\mathrm{x}$ & & SM & $\begin{array}{l}\text { Replaces widespread } C \text {. albus in NE Africa, but sympatric occurrence } \\
\text { over parts of their range with frequent hybridization in SE Highlands, } \\
\text { mixed pairs sporadically reported from most of the range } \\
\text { (del Hoyo et al. 2009). Details in Ash \& Atkins (2009: 372), further data } \\
\text { in Londei }(2005,2008) \text {. }\end{array}$ \\
\hline $20498 / 537$ & $\begin{array}{l}\text { Mirafra cantillans marginata } \\
\text { Hawker, } 1898\end{array}$ & $\mathrm{x}$ & & SM & $\begin{array}{l}\text { Patchily distributed in Ethiopia, apparently populations isolated from } \\
\text { each other but relationships between them unclear. Within Oromia only } \\
\text { in S (Borana) and NW (middle Awash area). Some movements, but not } \\
\text { understood (Ash \& Atkins 2009). }\end{array}$ \\
\hline $20502 / 538$ & $\begin{array}{l}\text { Mirafra albicauda } \\
\text { Reichenow, } 1891\end{array}$ & $?$ & & & $\begin{array}{l}\text { Patchily distributed from Chad to E and NE Africa, with some very iso- } \\
\text { lated populations. In Ethiopia records from only two 1-degree-tetrads } \\
\text { on Lake Abaya (one with about } 40 \% \text { area fraction in Oromia). Recently } \\
\text { only in Nechisar NP as rare visitor or possible resident, which lies just } \\
\text { outside Oromia (Ash \& Atkins 2009). Not included in Oromia checklist. }\end{array}$ \\
\hline $20511 / 539$ & $\begin{array}{l}\text { Mirafra pulpa } \\
\text { Friedmann, } 1930\end{array}$ & $\mathrm{x}$ & & SM & $\begin{array}{l}\text { Very locally distributed in Kenya and Ethiopia. The only Ethiopian re- } \\
\text { cord is from May } 1912 \text { in SW Oromia (specimen, Sagan river). Several } \\
\text { searches in the Sagan river area in the past years have not revealed the } \\
\text { species' presence (Ash \& Atkins 2009). }\end{array}$ \\
\hline
\end{tabular}




\begin{tabular}{|c|c|}
\hline $20519 / 541$ & $\begin{array}{l}\text { Mirafra hypermetra kathangorensis } \\
\text { Cave, } 1940\end{array}$ \\
\hline \multirow{2}{*}{$20521 / 541$} & Mirafra hypermetra gallarum \\
\hline & Hartert, 1907 \\
\hline \multirow[t]{2}{*}{$20523 / 540$} & Mirafra africana sharpii \\
\hline & Elliot, 1897 \\
\hline \multirow[t]{2}{*}{$20559 / 542$} & Mirafra rufocinnamomea torrida \\
\hline & Shelley, 1882 \\
\hline \multirow[t]{2}{*}{$20560 / 542$} & Mirafra rufocinnamomea rufocinnamomea \\
\hline & (Salvadori, 1865) \\
\hline \multirow{2}{*}{$20561 / 542$} & Mirafra rufocinnamomea omoensis \\
\hline & Neumann, 1928 \\
\hline \multirow{2}{*}{$20578 / 545$} & Mirafra collaris \\
\hline & Sharpe, 1896 \\
\hline \multirow[t]{2}{*}{$20583 /-$} & Heteromirafra archeri \\
\hline & Clarke, 1920 \\
\hline
\end{tabular}

SM Most likely M. h. kathangorensis in SW Ethiopia, incl. SW Oromia (SE Borana zone). Geographically isolated M. h. gallarum endemic to Ethiopia (Oromia, Afar and Somali Regional State), mainly in middle Awash area.

SM Most likely M. h. kathangorensis in SW Ethiopia, incl. SW Oromia (SE Borana zone). Geographically isolated M. h. gallarum endemic to Ethiopia (Oromia, Afar and Somali Regional State), mainly in middle Awash area.

One record of M. a. sharpii in E Ethiopia (Somali National Regional State) just outside of Oromia and some more on Somali side of the borState) just outside of Oromia and some more on Somali side of the bor-
der. Another recent record of one bird in August 2007 from Huri Hills on the Kenya side of the Oromia-Kenya border (Ash \& Atkins 2009); subspecies there unknown, but possibly M. a. athi. Not included in Oromia list.

SM Three subspecies of M. rufocinnamomea in Ethiopia and Oromia, whose distributional boundaries are poorly known. M. r. omoensis is found W and SW, M. r. torrida in S and nominate M. r. rufocinnamomea elsewhere, except SE (Ash \& Atkins 2009).

No record in Oromia so far, but can be expected at the southernmost edge

E, $\mathrm{H} \quad \mathrm{SM} \quad H$. archeri is one of the most endangered bird species in Africa. Its extinction in the near future must be feared if no serious protective measures are taken (Spottiswoode et al. 2009, Donald et al. 2010). According to recent studies, the species occurs not only in SE Oromia (Liben Plain near Negele Borana), but also near Jijiga (Somali Regional State). Recently it has been shown that H. sidamoensis Erard, 1975 and H. archeri form a single species (Spottiswoode et al. 2013).

Recent records between Turmi and Murle (Dowsett \& Dowsett-Lamaire 2015)

$20678 / 560$

Eremopterix nigriceps melanauchen

(Cabanis, 1851)

$20686 / 559$

Eremopterix signatus signatus

(Oustalet, 1886)

$20687 / 559$

Eremopterix signatus harrisoni

(Ogilvie-Grant, 1900)

$20706 / 550$

Ammomanes deserti assabensis

Salvadori, 1902

$20754 / 552$

Calandrella blanfordi daaroodensis

C. M. N. White, 1960

$20755 / 552$

Calandrella erlanger

(Neumann, 1906)

20762 / 553 Calandrella somalica megaensis

Benson, 1946

Calandrella somalica perconfusa

C. M. N. White, 1960

Spizocorys personata yavelloensis

(Benson, 1947)
SM C. alopex formerly placed in genus Mirafra. Treated conspecific with disjunct $C$. africanoides from S Africa. In E and S Oromia C. a. intercedens (Ash \& Atkins 2009). C. a. macdonaldi from Borana considered synonymous with C. a. intercedens (del Hoyo et al. 2004).

$\mathrm{H}+\quad \mathrm{SM}$ Most likely both subspecies in Ethiopia. Within Oromia E. s. signatus in NE (middle Awash) and maybe outermost S. Apparently E. s. harrisoni in SW Oromia (Ash \& Atkins 2009). Extent of the contact zone in the south, if any, is unclear.

SM

SM C. b. daaroodensis with only one occupied 1-minute-tetrad in easternmost Oromia (Ash \& Atkins 2009).

E, H AH Calandrella erlangeri formerly treated as C. blanfordi erlangeri. Within Ethiopia restricted to Amhara and C Oromia (Ash \& Atkins 2009).

O, E, H SM Two disjunct subspecies in Oromia: C. s. megaensis in S and C. s. perconfusa in NE (only two occupied 1-degree-tetrads). Nominate somalica from Somalia (and only one occupied record in E Somali Re-

H SM $\begin{aligned} & \text { gional State) also reported in SE highlands, but according to } \\ & \text { Ash \& Atkins (2009) no record has been traced. C. } \text { s. megaensis seems to }\end{aligned}$ Ash \& Atkins (2009) no record has been traced. C. S. megaensis seems to
be restricted to S Oromia, but with records along the Ethiopian-Kenyan border.

SM According to Ash \& Atkins (2009) there have been only five S. personata records (altogether 18 birds) in Ethiopia in more than 110 years. In Kenya only three sites are known. That makes worldwide only nine known locations with four subspecies. The range is said to be $350000 \mathrm{~km}^{2}$ (Birdlife International 2017), but probably much smaller and fragmented. The Birdlife map shows a range divided in three large coherent parts, which do probably not exist in this way. There is need for research, not least because the future outlook for this species could be research, not least because the future outlook for
critical due to climate change. In southern Ethiopia

S. p. yavelloensis has been scientifically described from the Yabello area around 70 years ago. Since then apparently no other reliable data exist on the presence of this form near its locus typicus. A search in June 2011 confimed, however, the presence of the birds in an area about $80 \mathrm{~km}$ west of Yabello (K. Gedeon, pers. obs.) 
$20812 / 555$

$20861 / 557$

$20862 / 557$

20863 / 557

$20865 / 557$

$21041 / 521$

21042 / 52

$21066 / 523$

$21082 /$

530-531

$21085 /$

530-531

$21086 /$

530-531

$21089 /$

530-531

$21109 / 525$

$21112 / 526$

$21113 / 526$

$5134 / 528$

$21134 / 528$

$21147 / 535$

$21150 / 535$

$21151 / 535$

$21172 / 534$

(Bannerman, 1923)

-/536 Petrochelidon sp.

Benson, 1946

Reichenow, 1907

(Blanford, 1869)

Benson, 1947

(Cabanis, 1850)

Reichenow, 1920

Reichenow, 1905

(Cabanis, 1850)

(Reichenow, 1905)

(Zedlitz, 1908)

Vincent, 1933

Neumann, 1904

Blanford, 1869

Hirundo megaensis

Benson, 1942

(Temminck, 1835)

Rüppell, 1845
Pseudalaemon fremantlii megaensis

Galerida cristata somaliensis

Galerida theklae praetermissa

Galerida theklae huei

Érard \& de Naurois, 1973

Galerida theklae huriensis

Galerida theklae harrarensis

Érard \& Jarry, 1973

Riparia paludicola minor

Riparia paludicola schoensis

Riparia cincta erlangeri

Ptyonoprogne fuligula obsoleta

Ptyonoprogne fuligula arabica

Ptyonoprogne fuligula pusilla

Ptyonoprogne fuligula fusciventris

Hirundo lucida rothschildi

Hirundo aethiopica aethiopica

Hirundo aethiopica amadoni

C. M. N. White, 1956

Cecropis daurica rufula

Cecropis daurica melanocrissus

Cecropis daurica domicella

(Hartlaub \& Finsch, 1870)

Cecropis senegalensis saturatior $\mathrm{x}$

$\mathrm{x}$

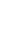

SM P. fremantlii restricted to $\mathrm{E}$ and NE Africa with three disjunct subspecies. P. f. megaensis geographically isolated in S Oromia (Borana) and N Kenya.

SM Subspecific taxonomy extremely complex. According to

Clements et al. (2015) 37 subspecies recognized, but that number could be provisional (cf. del Hoyo et al. 2004). In SE Oromia

G. c. somaliensis. This population (extending to N Kenya) is geographically widely separated from $G$. c. somaliensis, the same subspecies, in $\mathrm{N}$ Somali. Such a curious distribution raises doubts about the relative subspecific identification. Up to now no specimen from SE Ethiopia has been examined (Ash \& Atkins 2009).

AH Five subspecies of $G$. theklae in Ethiopia, four of them in Oromia: G. t. praetermissa in C Oromia, G. t. huei in Bale and Arusi Mts., G. t. harrarensis in NE, and G. t. huriensis in outermost S. Only

$\mathrm{x} \quad \mathrm{O}, \mathrm{E}, \mathrm{H} \quad \mathrm{AH} \quad \begin{aligned} & \text { G.t.praetermissa and G. t. huei with contact zone, though G. } t \text {. hu hu } \\ & \text { higher elevations. Other subspecies disjunct (Ash \& Atkins 2009). }\end{aligned}$

SM

E, $\mathrm{H} \quad \mathrm{SM}$

SG Two subspecies in Ethiopia: R. p. minor in NW, R. p. schoensis further south. The latter seems to be restricted to Ethiopia, but no information about their relative distribution (Ash \& Atkins 2009). Maybe R. p. minor does not reach Oromia.

P. fuligula and P. obsoleta treated as different species by

Ash \& Atkins (2009). Three or four subspecies in Ethiopia and Oromia: $P$. f. arabica and possibly P. o. obsoleta considered as migrant overwintering in Ethiopia (Ash \& Atkins 2009). P. f. pusilla is a breeding resident in N, C and E, P. t. fusciventris a breeding resident in C, W, S. There seems to be some geographical overlapping between these subspecies. More study is required to clarify the rather confused situation, as particularly in the west the taxa involved are unknown (Ash \& Atkins 2009).
E, $\mathrm{H}$

Two subspecies in Ethiopia and Oromia: H. a. aethiopica scattered over $\mathrm{N}, \mathrm{W}$ and S. H. a. amadoni in E. The latter with only one 1-degree-tetrad in NE Oromia (Ash \& Atkins 2009).

$\mathrm{H}+\quad \mathrm{SM}$

O, E, H SM Endemic to Oromia and restricted mainly to the Borana zone in SE Ethiopia (Mellanby et al. 2008). Biology and habitat requirements poorly understood (Bladon et al. 2015). From the eastern part of the range there are only a few records (Negele in the Guji zone,

Gabremichael et al. 2009, Bladon et al. 2015). Studies needed.

Three subspecies in Ethiopia and Oromia: Palaearctic C. d. rufula overwinters, but is difficult to separate from breeding resident

C. d. melanocrissus. The latter is nearly restricted to Ethiopia (mainly Oromia, Amhara, Tigre), with seven occupied 1-degree-tetrads in W Eritrea. Distributed over most of Oromia, but only scattered in SE C.d. domicella with only two occupied tetrads in E, Baro river area C.d. domicella with only
(Ash \& Atkins 2009). SG

Unidentified species with several records from Oromia (Ash \& Atkins 2009). Not included in the Oromia checklist. 


\begin{tabular}{|c|c|c|c|c|c|}
\hline $21211 / 532$ & $\begin{array}{l}\text { Delichon urbicum urbicum } \\
\text { (Linnaeus, 1758) }\end{array}$ & \multicolumn{3}{|l|}{$\mathrm{x}$} & \multirow[t]{2}{*}{$\begin{array}{l}\text { D. u. urbicum in Ethiopia and Oromia, D. u. meridionale possible } \\
\text { (Ash \& Atkins 2009). }\end{array}$} \\
\hline $21211 / 532$ & $\begin{array}{l}\text { Delichon urbicum meridionale } \\
\text { (Hartert, 1910) }\end{array}$ & \multicolumn{3}{|l|}{$?$} & \\
\hline $21235 / 519$ & $\begin{array}{l}\text { Psalidoprocne pristoptera pristoptera } \\
\text { (Rüppell, 1836) }\end{array}$ & $\mathrm{x}$ & $\mathrm{H}$ & & \multirow{8}{*}{$\begin{array}{l}\text { Four subspecies of } P \text {. pristoptera in Ethiopia and Oromia, but distribu- } \\
\text { tional boundaries poorly known. Within Oromia P.p. pristoptera in NW, } \\
P . \text { p. blanfordi and P. p. oleaginea in W, and P. p. antinori elsewhere, } \\
\text { except in far E (Ash \& Atkins 2009). Intermingling to a large extent } \\
\text { (Ash \& Atkins 2009), but apparently little interbreeding } \\
\text { (del Hoyo et al. 2004). Hence, blanfordi, antinorii and oleaginea some- } \\
\text { times treated as full species. Further work required, but distinguishing } \\
\text { the different subspecies in the field almost impossible. }\end{array}$} \\
\hline $21236 / 519$ & $\begin{array}{l}\text { Psalidoprocne pristoptera blanfordi } \\
\text { Blundell \& Lovat, } 1899\end{array}$ & $\mathrm{x}$ & $\mathrm{E}, \mathrm{H}$ & & \\
\hline \multirow[t]{2}{*}{$21237 / 519$} & Psalidoprocne pristoptera antinorii & $\mathrm{x}$ & $\mathrm{H}^{+}$ & & \\
\hline & Salvadori, 1884 & & & & \\
\hline \multirow{2}{*}{$21238 / 519$} & Psalidoprocne pristoptera oleaginea & $\mathrm{x}$ & $\mathrm{E}, \mathrm{H}$ & & \\
\hline & Neumann, 1904 & & & & \\
\hline \multirow[t]{2}{*}{$21563 / 514$} & Melaniparus guineensis & \multirow[t]{2}{*}{$\mathrm{x}$} & & & \\
\hline & (Shelley, 1900) & & & & \\
\hline $21565 / 514$ & $\begin{array}{l}\text { Melaniparus leucomelas leucomelas } \\
\text { (Rüppell, 1840) }\end{array}$ & $\mathrm{x}$ & $\mathrm{H}$ & $\mathrm{AH}$ & $\begin{array}{l}\text { M. l. leucomelas in NE Ethiopia and Eritrea geographically far separated } \\
\text { from } M \text {. l. insignis in Uganda and further south. In NE Oromia across } \\
\text { the Harar Mts. The Harar population seems also separated from popula- } \\
\text { tions in W Amhara, Tigre and Eritrea. However, distribution in some } \\
\text { parts uncertain, e.g. dark-eyed nominate leucomelas seen as far south } \\
\text { at Lake Langano (Ash \& Atkins } 2009 \text { ). Very different map in Redman } \\
\text { et al. (2011). Former subspecies M. l. guineensis now considered a spe- } \\
\text { cies on its own. }\end{array}$ \\
\hline $21587 / 516$ & $\begin{array}{l}\text { Melaniparus thruppi thruppi } \\
\text { (Shelley, 1885) }\end{array}$ & $\mathrm{x}$ & $\mathrm{H}$ & SM & \multirow[t]{2}{*}{$\begin{array}{l}\text { Two subspecies of M. thruppi in Ethiopia and Oromia. M. t. thruppi in E, } \\
\text { M. t. barakae in S, but dividing line between them uncertain } \\
\text { (Ash \& Atkins 2009). Not clear if they are spatially isolated or if a con- } \\
\text { tact zone exists in SE Oromia. }\end{array}$} \\
\hline $21588 / 519$ & $\begin{array}{l}\text { Melaniparus thruppi barakae } \\
\text { (F. J. Jackson, 1899) }\end{array}$ & $\mathrm{x}$ & $\mathrm{H}$ & SM & \\
\hline $21873 / 649$ & $\begin{array}{l}\text { Salpornis salvadori erlangeri } \\
\text { Neumann, } 1907\end{array}$ & $\mathrm{x}$ & $\mathrm{E}, \mathrm{H}$ & & $\begin{array}{l}\text { Within Ethiopia centre of distribution in Oromia. The Ethiopian subspe- } \\
\text { cies spatially separated from other African populations, next in W Kenya } \\
(S . \text { s. salvadori). Poorly known in Ethiopia. There are clear morpho- } \\
\text { logical and vocal differences that separate } S \text {. } s \text {. erlangeri from the other } \\
\text { groups (Tietze \& Martens } 2010 \text { ). Further studies should clarify whether } \\
\text { S. s. erlangeri is even a separate species. }\end{array}$ \\
\hline $22549 / 589$ & $\begin{array}{l}\text { Atimastillas flavicollis soror } \\
\text { (Neumann, 1914) }\end{array}$ & $\mathrm{x}$ & & & $\begin{array}{l}\text { Birds from Ethiopia are provisionally identified as } A . f \text {. soror, but are } \\
\text { greener on the back (Ash \& Atkins 2009). }\end{array}$ \\
\hline $22721 / 587$ & $\begin{array}{l}\text { Pycnonotus barbatus schoanus } \\
\text { Neumann, } 1905\end{array}$ & $\mathrm{x}$ & $\mathrm{H}+$ & & $\begin{array}{l}\text { Four subspecies in Ethiopia and Oromia. } P . b \text {. schoanus in } \mathrm{N}, \mathrm{C} \text { and } \mathrm{W} \text {, } \\
P . b \text {. somaliensis in NE, } P \text {. b. spurius in } \mathrm{S} \text {, and } P \text {. } b \text {. dodsoni in } \mathrm{S} \text { and } \\
\text { E. Interbreed where they meet. Subspeciation is not clear-cut, owing to }\end{array}$ \\
\hline $22722 / 587$ & $\begin{array}{l}\text { Pycnonotus barbatus somaliensis } \\
\text { Reichenow, } 1905\end{array}$ & $\mathrm{x}$ & $\mathrm{H}^{+}$ & & $\begin{array}{l}\text { (a) intersubspecific cross-breeding, (b) much overlap in distribution, } \\
\text { (c) different subspecies occurring in different ecological zones, and } \\
\text { (d) some wandering (Ash \& Atkins 2009). }\end{array}$ \\
\hline $22723 / 587$ & Pycnonotus barbatus dodsoni & $\mathrm{x}$ & $\mathrm{H}$ & SM & \\
\hline & Sharpe, 1895 & & & & \\
\hline $22725 / 587$ & Pycnonotus barbatus spurius & $\mathrm{x}$ & $\mathrm{H}^{+}$ & SM & \\
\hline & Reichenow, 1905 & & & & \\
\hline $23029 / 621$ & Sylvietta brachyura leucopsis & $\mathrm{x}$ & & & \\
\hline & (Reichenow, 1879) & & & & \\
\hline $23029 / 622$ & Sylvietta whytii loringi & $\mathrm{x}$ & & & \\
\hline & Mearns, 1911 & & & & \\
\hline $23048 / 595$ & $\begin{array}{l}\text { Melocichla mentalis amauroura } \\
\text { (Pelzeln, 1883) }\end{array}$ & $\mathrm{x}$ & & & \\
\hline $23234 / 613$ & $\begin{array}{l}\text { Phylloscopus umbrovirens omoensis } \\
\text { (Neumann, 1905) }\end{array}$ & $\mathrm{x}$ & $\mathrm{O}, \mathrm{E}, \mathrm{H}$ & $\mathrm{AH}$ & $\begin{array}{l}\text { P. u. omoensis endemic to Ethiopia and nearly restricted to mountainous } \\
\text { areas of Oromia, with only three occupied 1-degree-tetrads outside } \\
\text { (Ash \& Atkins 2009). }\end{array}$ \\
\hline $23245 / 614$ & $\begin{array}{l}\text { Phylloscopus trochilus acredula } \\
\text { (Linnaeus, 1758) }\end{array}$ & $\mathrm{x}$ & & & $\begin{array}{l}\text { Three subspecies in Ethiopia and most likely in Oromia. All are Pa- } \\
\text { laearctic passage migrants and winter visitors. Subspecific abundance } \\
\text { poorly understood, but most birds are probably P. } \text { t. acredula }\end{array}$ \\
\hline $23246 / 614$ & Phylloscopus trochilus trochilus & $\mathrm{x}$ & & & (Ash \& Atkins 2009). \\
\hline & (Linnaeus, 1758) & & & & \\
\hline $23247 / 614$ & Phylloscopus trochilus yakutensis & $\mathrm{x}$ & & & \\
\hline & Ticehurst, 1935 & & & & \\
\hline
\end{tabular}




\begin{tabular}{|c|c|c|c|c|c|}
\hline $23249 / 615$ & $\begin{array}{l}\text { Phylloscopus collybita abietinus } \\
\text { (Nilsson, 1819) }\end{array}$ & $\mathrm{x}$ & & & $\begin{array}{l}\text { Two subspecies in Ethiopia and most likely in Oromia. Both are Pa- } \\
\text { laearctic passage migrants and winter visitors (Ash \& Atkins 2009). }\end{array}$ \\
\hline $23250 / 615$ & $\begin{array}{l}\text { Phylloscopus collybita collybita } \\
\text { (Vieillot, 1817) }\end{array}$ & $\mathrm{x}$ & & & \multirow{4}{*}{$\begin{array}{l}\text { Two subspecies in Ethiopia and Oromia. Both overwintering migrants } \\
\text { and presumably on passage. Most birds are Palaearctic I. p. elaeica. In } \\
\text { Oromia I. p. pallida (ssp. breeding in Egypt) has been recorded only in } \\
\text { one 1-degree-tetrad, on the westernmost fringe (Ash \& Atkins 2009). }\end{array}$} \\
\hline $23470 / 607$ & $\begin{array}{l}\text { Iduna pallida elaeica } \\
\text { (Lindermayer, A, 1843) }\end{array}$ & $\mathrm{x}$ & & & \\
\hline $23471 / 607$ & $\begin{array}{l}\text { Iduna pallida pallida } \\
\text { (Hemprich \& Ehrenberg, 1833) }\end{array}$ & $\mathrm{x}$ & & & \\
\hline $23478 / 611$ & $\begin{array}{l}\text { Iduna natalensis massaica } \\
\text { (Fischer, GA \& Reichenow, 1884) }\end{array}$ & $\mathrm{x}$ & & & \\
\hline $23509 / 604$ & $\begin{array}{l}\text { Acrocephalus scirpaceus fuscus } \\
\text { (Hemprich \& Ehrenberg, 1833) }\end{array}$ & $\mathrm{x}$ & & & \multirow[t]{2}{*}{$\begin{array}{l}\text { Most Palaearctic migrants in Ethiopia are } A \text {. s. fuscus, but } \\
\text { A. s. scirpaceus may occur (Ash \& Atkins 2009). }\end{array}$} \\
\hline $23514 / 604$ & $\begin{array}{l}\text { Acrocephalus baeticatus cinnamomeus } \\
\text { Reichenow, } 1908\end{array}$ & $\mathrm{x}$ & & & \\
\hline $23522 / 600$ & $\begin{array}{l}\text { Acrocephalus gracilirostris jacksoni } \\
\text { (Neumann, 1901) }\end{array}$ & $\mathrm{x}$ & & & \multirow[t]{2}{*}{$\begin{array}{l}\text { Two subspecies in Ethiopia and Oromia. Within Oromia A. g. jacksoni } \\
\text { on the westernmost fringe (one occupied 1-degree-tetrad), A. g. parvus } \\
\text { along the Rift Valley and Awash (Ash \& Atkins 2009). }\end{array}$} \\
\hline $23525 / 600$ & $\begin{array}{l}\text { Acrocephalus gracilirostris parvus } \\
\text { (Fischer, GA \& Reichenow, 1884) }\end{array}$ & $\mathrm{x}$ & & & \\
\hline $23539 / 601$ & $\begin{array}{l}\text { Acrocephalus arundinaceus arundinaceus } \\
\text { (Linnaeus, 1758) }\end{array}$ & $\mathrm{x}$ & & & \multirow[t]{2}{*}{$\begin{array}{l}\text { Two subspecies in Ethiopia and Oromia. Both Palaearctic migrants and } \\
\text { overwintering in small numbers, A. a. arundinaceus predominate } \\
\text { (Ash \& Atkins 2009). }\end{array}$} \\
\hline $23540 / 601$ & $\begin{array}{l}\text { Acrocephalus arundinaceus zarudnyi } \\
\text { Hartert, } 1907\end{array}$ & $\mathrm{x}$ & & & \\
\hline $23654 / 591$ & $\begin{array}{l}\text { Schoenicola brevirostris alexinae } \\
\text { (Heuglin, 1863) }\end{array}$ & $\mathrm{x}$ & & & Last recorded in 1927. Further studies needed. \\
\hline $23689 / 592$ & $\begin{array}{l}\text { Bradypterus baboecala sudanensis } \\
\text { Grant \& Mackworth-Praed, } 1941\end{array}$ & $\mathrm{x}$ & & & \multirow{2}{*}{$\begin{array}{l}\text { Two subspecies in Ethiopia and Oromia. Within Oromia B. b. sudanensis } \\
\text { in the westernmost fringe (one occupied 1-degree-tetrad), B. b. abyssini- } \\
\text { cus scattered in other parts, with six occupied 1-degree-tetrads in Oromia } \\
\text { (Ash \& Atkins 2009). }\end{array}$} \\
\hline $23690 / 592$ & $\begin{array}{l}\text { Bradypterus baboecala abyssinicus } \\
\text { (Blundell \& Lovat, 1899) }\end{array}$ & $\mathrm{x}$ & E, $\mathrm{H}$ & & \\
\hline $23719 / 598$ & $\begin{array}{l}\text { Locustella luscinioides luscinioides } \\
\text { (Savi, 1824) }\end{array}$ & $\mathrm{x}$ & & & \multirow[t]{2}{*}{$\begin{array}{l}\text { Three subspecies in Ethiopia and most likely in Oromia. All are Pa- } \\
\text { laearctic overwinterers and difficult to observe. Only two occupied } \\
\text { 1-degree-terads in Oromia (Ash \& Atkins 2009). }\end{array}$} \\
\hline $23720 / 598$ & $\begin{array}{l}\text { Locustella luscinioides sarmatica } \\
\text { Kazakov, } 1973\end{array}$ & $\mathrm{x}$ & & & \\
\hline $23721 / 598$ & $\begin{array}{l}\text { Locustella luscinioides fusca } \\
\text { (Severtsov, 1873) }\end{array}$ & $\mathrm{x}$ & & & \multirow{6}{*}{$\begin{array}{l}\text { Three subspecies in Ethiopia and Oromia. Within Ethiopia } \\
\text { A.f. flavocincta in S, A.f. viridiceps in NE (only two occupied 1-degree- } \\
\text { tetrads), and } A \text {. } f \text {. abyssinica in W. Core range of the latter in Oromia } \\
\text { (Ash \& Atkins 2009). }\end{array}$} \\
\hline $23730 / 596$ & $\begin{array}{l}\text { Locustella naevia straminea } \\
\text { Seebohm, } 1881\end{array}$ & $\mathrm{x}$ & & & \\
\hline $23851 / 583$ & Apalis flavida viridiceps & $\mathrm{x}$ & $\mathrm{H}^{+}$ & SM & \\
\hline $23852 / 583$ & $\begin{array}{l}\text { Apalis flavida flavocincta } \\
\text { (Sharpe, 1882) }\end{array}$ & $\mathrm{x}$ & & SM & \\
\hline $23855 / 583$ & $\begin{array}{l}\text { Apalis flavida abyssinica } \\
\text { Érard, } 1974\end{array}$ & $\mathrm{x}$ & E, $\mathrm{H}$ & & \\
\hline $23924 / 585$ & $\begin{array}{l}\text { Camaroptera brachyura abessinica } \\
\text { Zedlitz, } 1911\end{array}$ & $\mathrm{x}$ & & & \\
\hline $23949 / 584$ & $\begin{array}{l}\text { Urorhipis rufifrons rufifrons } \\
\text { (Rüppell, 1840) }\end{array}$ & $?$ & & & \multirow[t]{2}{*}{$\begin{array}{l}\text { Two subspecies in Ethiopia. Within Oromia } U . r \text { smithi in E and S, } \\
\text { U. r. rufifrons could be expected in the NE corner. Division line between } \\
\text { the two subspecies unknown (Ash \& Atkins 2009). }\end{array}$} \\
\hline $23950 / 584$ & $\begin{array}{l}\text { Urorhipis rufifrons smithi } \\
\text { (Sharpe, 1895) }\end{array}$ & $\mathrm{x}$ & & SM & \\
\hline
\end{tabular}




\begin{tabular}{|c|c|c|c|c|c|}
\hline $23998 / 561$ & $\begin{array}{l}\text { Cisticola erythrops pyrrhomitra } \\
\text { Reichenow, } 1916\end{array}$ & $\mathrm{x}$ & & & $\begin{array}{l}\text { Two subspecies in Ethiopia. Within Oromia C. e. pyrrhomitra in C and } \\
\text { W. C. e. niloticus could be expected in the outermost NW (Ash \& Atkins } \\
\text { 2009). }\end{array}$ \\
\hline $23999 / 561$ & $\begin{array}{l}\text { Cisticola erythrops niloticus } \\
\text { Madarász, } 1914\end{array}$ & ? & & & \\
\hline $24006 / 562$ & $\begin{array}{l}\text { Cisticola cantans cantans } \\
\text { (Heuglin, 1869) }\end{array}$ & $\mathrm{x}$ & E, $\mathrm{H}$ & & $\begin{array}{l}\text { C. c. cantans nearly restricted to Ethiopia, core range in Oromia } \\
\text { (Ash \& Atkins 2009). }\end{array}$ \\
\hline $24042 / 564$ & $\begin{array}{l}\text { Cisticola bodessa bodessa } \\
\text { Mearns, } 1913\end{array}$ & $\mathrm{x}$ & & SM & $\begin{array}{l}\text { Two subspecies in Ethiopia and Oromia. C. } \text { b. bodessa in E and S Oro- } \\
\text { mia with offshoots to Kenya, C. b. kaffensis in SW Oromia, W of Rift } \\
\text { Valley, where it could reach S Sudan and Kenya }\end{array}$ \\
\hline $24043 / 564$ & $\begin{array}{l}\text { Cisticola bodessa kaffensis } \\
\text { Érard, } 1974\end{array}$ & $\mathrm{x}$ & & SM & $\begin{array}{l}\text { (Ash \& Atkins 2009). However, the distribution of the subspecies some- } \\
\text { what unclear. According to del Hoyo et al. (2006) C. b. bodessa also in } \\
\text { Eritrea and SE Sudan. Further clarification needed. }\end{array}$ \\
\hline $24046 / 563$ & $\begin{array}{l}\text { Cisticola chiniana fricki } \\
\text { Mearns, } 1913\end{array}$ & $\mathrm{x}$ & & SM & \\
\hline $24063 / 565$ & $\begin{array}{l}\text { Cisticola cinereolus cinereolus } \\
\text { Salvadori, } 1888\end{array}$ & $\mathrm{x}$ & $\mathrm{H}$ & SM & $\begin{array}{l}\text { Two subspecies in Ethiopia and Oromia. Within Oromia disjunct distri- } \\
\text { bution, C. c. cinereolus in NE, C. c. schillingsi in S } \\
\text { (Ash \& Atkins 2009). }\end{array}$ \\
\hline 24064 / 565 & $\begin{array}{l}\text { Cisticola cinereolus schillingsi } \\
\text { Reichenow, } 1905\end{array}$ & $\mathrm{x}$ & & SM & \\
\hline $24068 / 569$ & $\begin{array}{l}\text { Cisticola ruficeps mongalla } \\
\text { Lynes, } 1930\end{array}$ & $\mathrm{x}$ & & SG & $\begin{array}{l}\text { Ash \& Atkins (2009) list only three records of C. r. mongalla from } \\
\text { Ethiopa. Two recent observations, possibly of mongalla, from Sala river } \\
\text { (Rannestad 2016). }\end{array}$ \\
\hline $24100 / 566$ & $\begin{array}{l}\text { Cisticola galactotes marginatus } \\
\text { (Heuglin, 1869) }\end{array}$ & $\mathrm{x}$ & & & $\begin{array}{l}\text { Two subspecies in Ethiopia and Oromia: } C . \text { g. marginatus (sometimes } \\
\text { considered a separate species C. marginatus) in westernmost Oromia, } \\
\text { and } C . \text { g. lugubris (sometimes considered a separate species C. lugubris) }\end{array}$ \\
\hline $24108 / 566$ & $\begin{array}{l}\text { Cisticola galactotes lugubris } \\
\text { (Rüppell, 1840) }\end{array}$ & $\mathrm{x}$ & E, $\mathrm{H}$ & & $\begin{array}{l}\text { widespread in Oromia, except S and SE. The latter nearly endemic to } \\
\text { Ethiopia, with few occupied 1-degree-tetrads in Eritrea } \\
\text { (Ash \& Atkins 2009). }\end{array}$ \\
\hline $24125 / 567$ & $\begin{array}{l}\text { Cisticola robustus schraderi } \\
\text { Neumann, } 1906\end{array}$ & $\mathrm{x}$ & & & $\begin{array}{l}\text { Three subspecies in Ethiopia and Oromia. Within Oromia C. r. schraderi } \\
\text { in NW (only } 1 \text { occupied } 1 \text {-degree). Records near the Sudan border pecu- } \\
\text { liarly geographically isolated from schraderi records from Eritrea and } \mathrm{N}\end{array}$ \\
\hline $24126 / 567$ & $\begin{array}{l}\text { Cisticola robustus robustus } \\
\text { (Rüppell, 1845) }\end{array}$ & $\mathrm{x}$ & E, $\mathrm{H}$ & & $\begin{array}{l}\text { Ethiopia. C. r. robustus in N and E, and C.r. omo in W (nearly restricted } \\
\text { to Oromia). Birds in lower Rift Valley remain subspecifically unidenti- } \\
\text { fied (Ash \& Atkins 2009). }\end{array}$ \\
\hline $24127 / 567$ & $\begin{array}{l}\text { Cisticola robustus omo } \\
\text { Neumann \& Lynes, } 1928\end{array}$ & $\mathrm{x}$ & $\mathrm{O}, \mathrm{E}, \mathrm{H}$ & & \\
\hline $24135 / 568$ & $\begin{array}{l}\text { Cisticola natalensis inexpectatus } \\
\text { Neumann, } 1906\end{array}$ & $\mathrm{x}$ & E, $\mathrm{H}$ & & $\begin{array}{l}\text { Two subspecies in Ethiopia and Oromia. Within Oromia } \\
\text { C. } n \text {. inexpectatus scattered in W, C and E, core range of this subspecies } \\
\text { in Oromia. C. } n \text {. argenteus in SE (Ash \& Atkins 2009). }\end{array}$ \\
\hline $24136 / 568$ & $\begin{array}{l}\text { Cisticola natalensis argenteus } \\
\text { Reichenow, } 1905\end{array}$ & $\mathrm{x}$ & & SM & \\
\hline $24155 / 571$ & $\begin{array}{l}\text { Cisticola brachypterus zedlitzi } \\
\text { Reichenow, } 1909\end{array}$ & $\mathrm{x}$ & E, $\mathrm{H}$ & & $\begin{array}{l}\text { C. b. zedlitzi nearly restricted to Ethiopia, with core range in Oromia } \\
\text { (Ash \& Atkins 2009). }\end{array}$ \\
\hline $24166 / 572$ & $\begin{array}{l}\text { Cisticola troglodytes ferrugineus } \\
\text { Heuglin, } 1864\end{array}$ & $\mathrm{x}$ & & SG & \\
\hline $24186 / 573$ & $\begin{array}{l}\text { Cisticola juncidis uropygialis } \\
\text { (Fraser, 1843) }\end{array}$ & $\mathrm{x}$ & & & \\
\hline $24192 / 574$ & $\begin{array}{l}\text { Cisticola aridulus lavendulae } \\
\text { Ogilvie-Grant \& Reid, } 1901\end{array}$ & $\mathrm{x}$ & $\mathrm{H}$ & SM & Scattered records. In Oromia in Awash region and S. \\
\hline $24220 / 576$ & $\begin{array}{l}\text { Cisticola brunnescens brunnescens } \\
\text { Heuglin, } 1862\end{array}$ & $\mathrm{x}$ & $\mathrm{H}$ & & \\
\hline $24382 / 578$ & $\begin{array}{l}\text { Prinia subflava subflava } \\
\text { (J. F. Gmelin, 1789) }\end{array}$ & $\mathrm{x}$ & & & $\begin{array}{l}\text { Two subspecies in Ethiopia and Oromia. Within Oromia P. s. subflava } \\
\text { widespread, except in S and SE, C. s. pallescens on the westernmost } \\
\text { fringe, with only three occupied 1-degree-tetrads (Ash \& Atkins 2009). }\end{array}$ \\
\hline $24383 / 578$ & $\begin{array}{l}\text { Prinia subflava pallescens } \\
\text { Madarász, } 1914\end{array}$ & $\mathrm{x}$ & & & \\
\hline $24404 / 579$ & $\begin{array}{l}\text { Prinia somalica erlangeri } \\
\text { Reichenow, } 1905\end{array}$ & $\mathrm{x}$ & & SM & $\begin{array}{l}\text { Two subspecies in Ethiopia and Oromia. Within Oromia P. s. erlangeri in } \\
\text { S and SE. P. s. somalica on the north-easternmost fringe, with only one } \\
\text { occupied 1-degree-tetrad (Ash \& Atkins 2009). }\end{array}$ \\
\hline $24405 / 579$ & Prinia somalica somalica & $\mathrm{x}$ & $\mathrm{H}$ & SM & \\
\hline
\end{tabular}




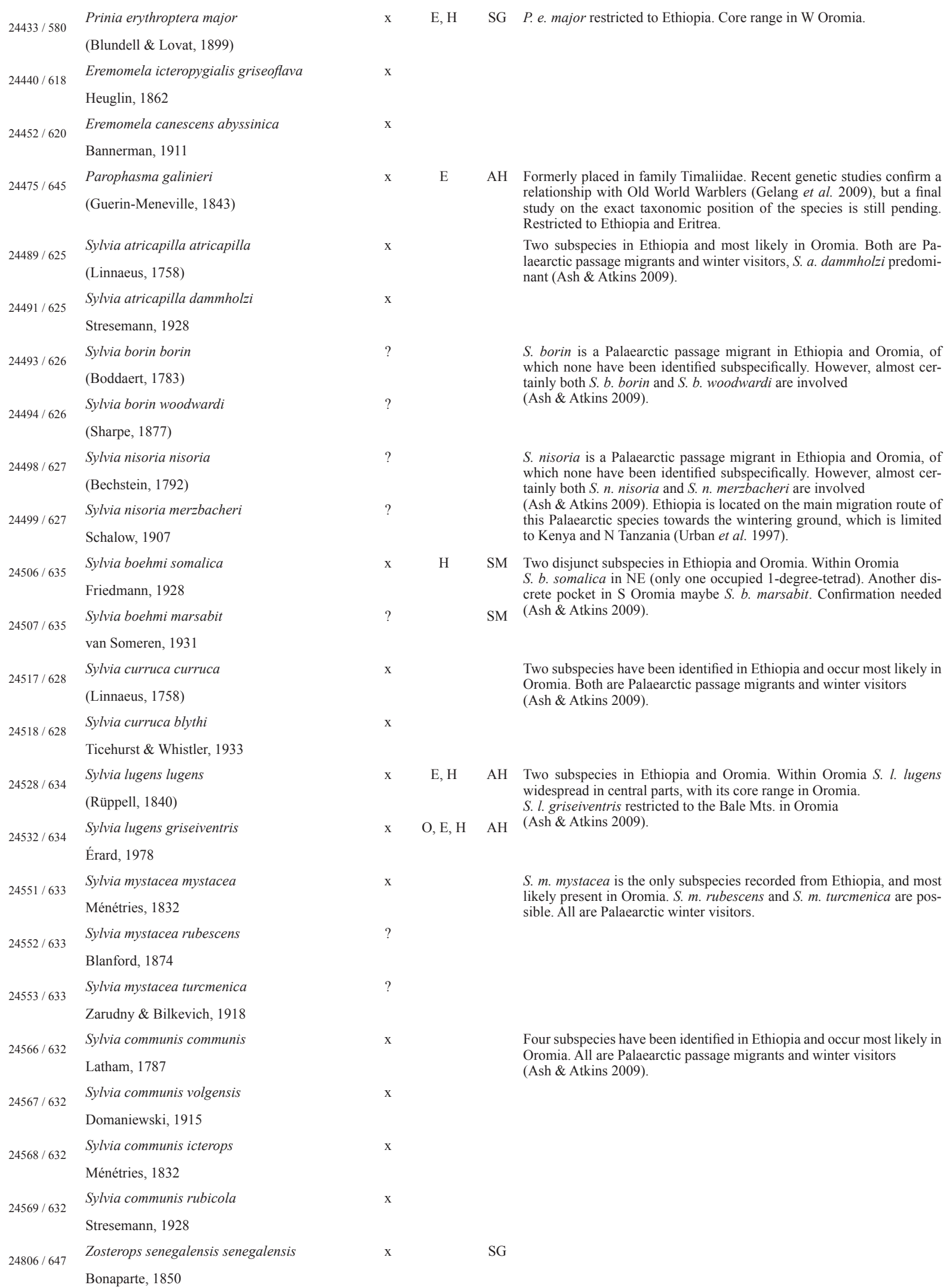




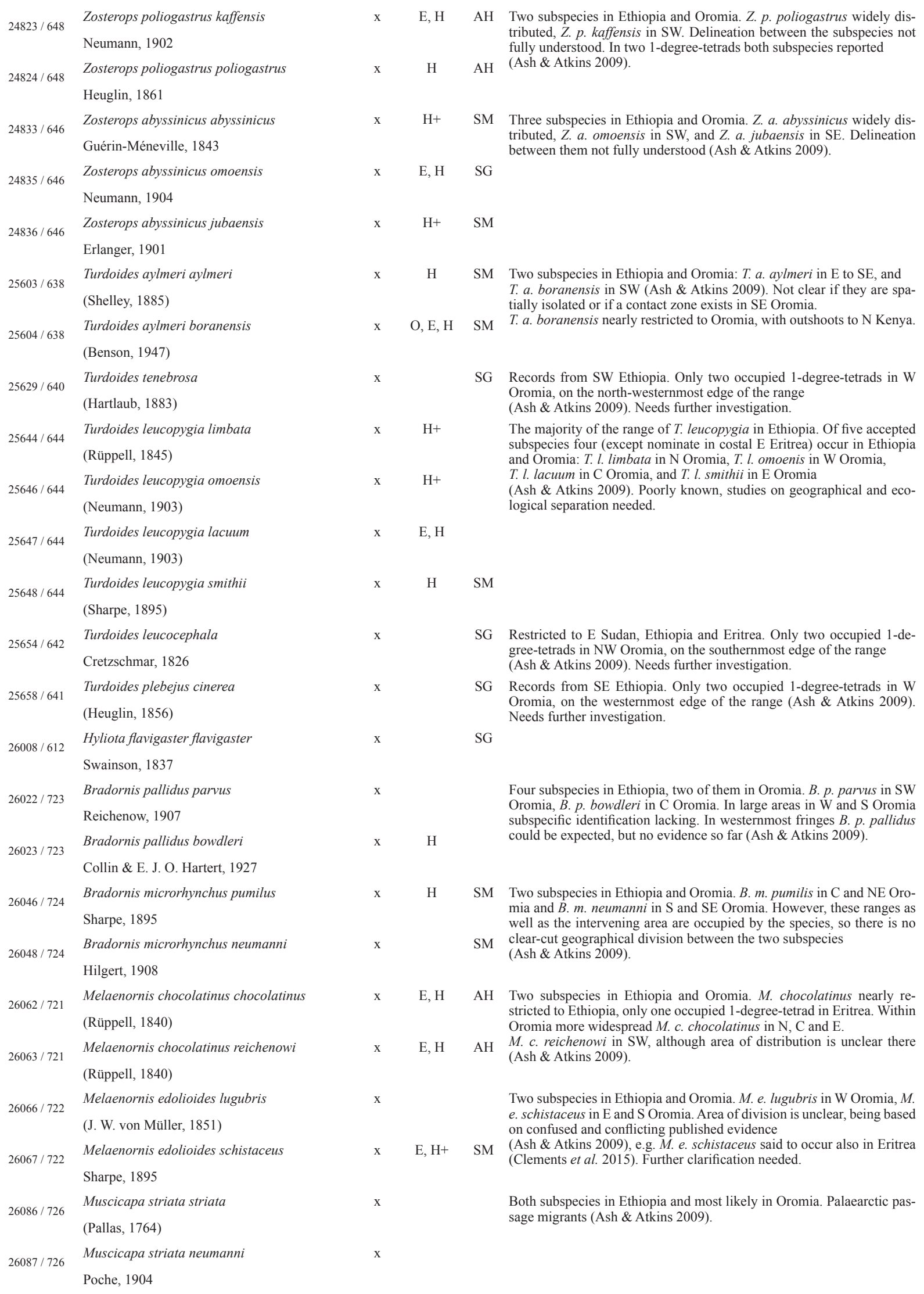




\begin{tabular}{|c|c|c|c|c|c|}
\hline $26132 / 728$ & $\begin{array}{l}\text { Muscicapa adusta minima } \\
\text { Heuglin, } 1862\end{array}$ & $\mathrm{x}$ & \multicolumn{2}{|l|}{ E, H } & $\begin{array}{l}\text { M. a. minima nearly restricted to Ethiopia with only } 3 \text { occupied 1-de- } \\
\text { gree-tetrads in Eritrea, core range in Oromia (Ash \& Atkins 2009). }\end{array}$ \\
\hline $26186 / 692$ & $\begin{array}{l}\text { Cercotrichas podobe podobe } \\
\text { (Statius Müller, 1776) }\end{array}$ & $\mathrm{x}$ & & & \multirow{2}{*}{$\begin{array}{l}\text { According to Ash \& Atkins (2009) C.p. podobe a non-breeding visitor to } \\
\text { Ethiopia. Could include Palaearctic migrants from W Arabia, where this } \\
\text { mainly African subspecies also occurs (del Hoyo et al. 2005). No clear } \\
\text { evidence for C. p. melanoptera from S Arabia. }\end{array}$} \\
\hline $26187 / 692$ & $\begin{array}{l}\text { Cercotrichas podobe melanoptera } \\
\text { (Hemprich \& Ehrenberg, 1833) }\end{array}$ & $?$ & & & \\
\hline $26190 / 691$ & $\begin{array}{l}\text { Cercotrichas galactotes syriacus } \\
\text { (Hemprich \& Ehrenberg, 1833) }\end{array}$ & $?$ & & & \multirow[t]{2}{*}{$\begin{array}{l}\text { C. g. familaris widespread Palaearctic visitor. C. g. syriacus visitor in } \\
\text { Eritrea and possibly in Ethiopia, but no clear evidence } \\
\text { (Ash \& Atkins 2009) }\end{array}$} \\
\hline $26191 / 691$ & $\begin{array}{l}\text { Cercotrichas galactotes familiaris } \\
\text { (Ménétriés, 1832) }\end{array}$ & $\mathrm{x}$ & & & \\
\hline $26193 / 691$ & $\begin{array}{l}\text { Cercotrichas minor minor } \\
\text { (Cabanis, } 1850)\end{array}$ & $\mathrm{x}$ & & & \multirow[t]{2}{*}{$\begin{array}{l}\text { C. m. minor breeding resident in SE Oromia. Treated by } \\
\text { del Hoyo et al. (2005) as Erythropygia galactotes minor, by } \\
\text { Ash \& Atkins (2009) as Cercostrichas galactotes minor. }\end{array}$} \\
\hline $26203 / 690$ & $\begin{array}{l}\text { Cercotrichas leucophrys leucoptera } \\
\text { (Rüppell, 1845) }\end{array}$ & $\mathrm{x}$ & & & \\
\hline $26450 / 684$ & $\begin{array}{l}\text { Cossypha semirufa semirufa } \\
\text { (Rüppell, 1840) }\end{array}$ & $\mathrm{x}$ & & $\mathrm{AH}$ & \multirow{3}{*}{$\begin{array}{l}\text { Two subspecies of C. semirufa in Ethiopia and Oromia. C. s. semirufa in } \\
\mathrm{N}, \mathrm{W} \text {, and S (most of the species range), and C. s. donaldsoni in C and } \\
\text { parts of E. Subspecies intergrade. C. s. donaldsoni is restricted to Ethio- } \\
\text { pia and nearly restricted to Oromia, with only one occupied 1-degree- } \\
\text { tetrad in Afar National Regional State (Ash \& Atkins 2009). }\end{array}$} \\
\hline $26451 / 684$ & $\begin{array}{l}\text { Cossypha semirufa donaldsoni } \\
\text { Sharpe, } 1895\end{array}$ & $\mathrm{x}$ & $\mathrm{O}, \mathrm{E}, \mathrm{H}$ & $\mathrm{AH}$ & \\
\hline $26459 / 686$ & $\begin{array}{l}\text { Cossypha natalensis intensa } \\
\text { Mearns, } 1913\end{array}$ & $\mathrm{x}$ & & & \\
\hline $26471 / 688$ & $\begin{array}{l}\text { Cossypha albicapillus omoensis } \\
\text { Sharpe, } 1900\end{array}$ & $\mathrm{x}$ & & SM & $\begin{array}{l}\text { C. a. omoensis with tiny range in SE South Sudan and SW Ethiopia. } \\
\text { Geographically very isolated from the two other subspecies (next popu- } \\
\text { lation in S Chad). In Oromia only two occupied } 1-\mathrm{km} \text {-tetrads on Lake } \\
\text { Chew Bahir. Largely a Sudan-Guinean species, but C. a. omoensis more } \\
\text { connected to the Somalia-Masai biome (cf. similar situation with Myr- } \\
\text { mecocichla albifrons pachyrhyncha). }\end{array}$ \\
\hline $26477 / 689$ & $\begin{array}{l}\text { Cichladusa guttata guttata } \\
\text { (Heuglin, 1862) }\end{array}$ & $\mathrm{x}$ & & & \multirow[t]{4}{*}{$\begin{array}{l}\text { All three subspecies of } C \text {. guttata in Ethiopia and Oromia. C. g. guttata } \\
\text { in SW, C. g. intercalas in S, and C. g. rufipennis in SE. Dividing line } \\
\text { between subspecies in Ash \& Atkins' (2009) map uncertain. }\end{array}$} \\
\hline $26478 / 689$ & Cichladusa guttata intercalans & $\mathrm{x}$ & & & \\
\hline & Clancey, 1986 & & & & \\
\hline $26479 / 689$ & $\begin{array}{l}\text { Cichladusa guttata rufipennis } \\
\text { Sharpe, } 1901\end{array}$ & $\mathrm{x}$ & & & \\
\hline $26615 / 682$ & $\begin{array}{l}\text { Luscinia megarhynchos megarhynchos } \\
\text { C. L. Brehm, } 1831\end{array}$ & $\mathrm{x}$ & & & \multirow[t]{3}{*}{$\begin{array}{l}\text { All three subspecies of L. megarhynchos are Palaearctic visitors and pas- } \\
\text { sage migrants (Ash \& Atkins 2009). L. m. golzii formerly named } \\
\text { L. m. hafizi Severtsov, } 1873 .\end{array}$} \\
\hline $26616 / 682$ & $\begin{array}{l}\text { Luscinia megarhynchos africana } \\
\text { (G. A. Fischer \& Reichenow, 1884) }\end{array}$ & $\mathrm{x}$ & & & \\
\hline $26617 / 682$ & $\begin{array}{l}\text { Luscinia megarhynchos golzii } \\
\text { Cabanis, } 1873\end{array}$ & $\mathrm{x}$ & & & \\
\hline $26624 / 680$ & $\begin{array}{l}\text { Luscinia svecica svecica } \\
\text { (Linnaeus, 1758) }\end{array}$ & $\mathrm{x}$ & & & \multirow{3}{*}{$\begin{array}{l}\text { Three subspecies of } L \text {. svecica are Palaearctic visitors and passage mi- } \\
\text { grants (Ash \& Atkins 2009). } \\
\text { Both subspecies of } P \text {. phoenicurus are Palaearctic visitors and passage } \\
\text { migrants (Ash \& Atkins 2009). }\end{array}$} \\
\hline $26634 / 680$ & $\begin{array}{l}\text { Luscinia svecica cyanecula } \\
\text { (Meisner, 1804) }\end{array}$ & $\mathrm{x}$ & & & \\
\hline $26636 / 680$ & $\begin{array}{l}\text { Luscinia svecica magna } \\
\text { (Zarudny \& Loudon, 1904) }\end{array}$ & $\mathrm{x}$ & & & \\
\hline $26813 / 694$ & $\begin{array}{l}\text { Phoenicurus phoenicurus phoenicurus } \\
\text { (Linnaeus, 1758) }\end{array}$ & $\mathrm{x}$ & & & \multirow[t]{3}{*}{$\begin{array}{l}\text { Both subspecies of } P \text {. phoenicurus are Palaearctic visitors and passage } \\
\text { migrants (Ash \& Atkins 2009). }\end{array}$} \\
\hline 26814 / 694 & $\begin{array}{l}\text { Phoenicurus phoenicurus samamisicus } \\
\text { (Hablizl, 1783) }\end{array}$ & $\mathrm{x}$ & & & \\
\hline 26827693 & $\begin{array}{l}\text { Phoenicurus ochruros phoenicuroides } \\
\text { (F. Moore, 1854) }\end{array}$ & $\mathrm{x}$ & & & \\
\hline
\end{tabular}




\begin{tabular}{|c|c|}
\hline $26855 / 720$ & $\begin{array}{l}\text { Monticola solitarius solitarius } \\
\text { (Linnaeus, 1758) }\end{array}$ \\
\hline $26856 / 720$ & $\begin{array}{l}\text { Monticola solitarius longirostris } \\
\text { (Blyth, 1847) }\end{array}$ \\
\hline $26877 /-$ & $\begin{array}{l}\text { Saxicola maurus hemprichii } \\
\text { Ehrenberg, } 1833\end{array}$ \\
\hline $26879 /-$ & $\begin{array}{l}\text { Saxicola maurus variegatus } \\
\text { (S. G. Gmelin, 1774) }\end{array}$ \\
\hline $26901 / 696$ & $\begin{array}{l}\text { Saxicola torquatus albofasciatus } \\
\text { Rüppell, } 1845\end{array}$ \\
\hline $26946 / 715$ & $\begin{array}{l}\text { Myrmecocichla albifrons pachyrhyncha } \\
\text { (Neumann, 1906) }\end{array}$ \\
\hline $26955 / 716$ & $\begin{array}{l}\text { Thamnolaea cinnamomeiventris albiscapulata } \\
\text { (Rüppell, 1837) }\end{array}$ \\
\hline $26956 / 716$ & $\begin{array}{l}\text { Thamnolaea cinnamomeiventris subrufipennis } \\
\text { Reichenow, } 1887\end{array}$ \\
\hline $26978 / 709$ & $\begin{array}{l}\text { Cercomela familiaris falkensteini } \\
\text { (Cabanis, 1875) }\end{array}$ \\
\hline $26979 / 709$ & $\begin{array}{l}\text { Cercomela familiaris omoensis } \\
\text { (Neumann, 1904) }\end{array}$ \\
\hline $26990 / 710$ & $\begin{array}{l}\text { Cercomela scotocerca turkana } \\
\text { van Someren, } 1920\end{array}$ \\
\hline $26992 / 710$ & $\begin{array}{l}\text { Cercomela scotocerca spectatrix } \\
\text { S. R. Clarke, } 1919\end{array}$ \\
\hline $26995 / 711$ & $\begin{array}{l}\text { Cercomela dubia } \\
\text { (Blundell \& Lovat, 1899) }\end{array}$ \\
\hline $26996 / 712$ & $\begin{array}{l}\text { Cercomela melanura lypura } \\
\text { (Hemprich \& Ehrenberg, 1833) }\end{array}$ \\
\hline $27003 / 713$ & $\begin{array}{l}\text { Cercomela sordida sordida } \\
\text { (Rüppell, 1837) }\end{array}$ \\
\hline $27023 / 701$ & Oenanthe phillipsi \\
\hline
\end{tabular}

(Shelley, 1885)

$27027 / 700$

$27028 / 700$

$27041 / 707$

$27054 / 705$

$27072 / 697$

$27073 / 698$

Oenanthe oenanthe oenanthe

(Linnaeus, 1758)

Oenanthe oenanthe libanotica

(Hemprich \& Ehrenberg, 1833)

Oenanthe lugubris lugubris

(Rüppell, 1837)

Oenanthe hispanica melanoleuca

(Güldenstädt, 1775)

Oenanthe bottae frenata

(Heuglin, 1869)

Oenanthe heuglini

(Finsch \& Hartlaub, 1870)
$?$

$\mathrm{x}$

$\mathrm{x}$

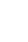

C. f. falkensteini recorded above about $11^{\circ} \mathrm{N}$ (outside Oromia), C. f. omoensis in SW Oromia and further SW. In the intervening area, and therefore in most parts of Oromia, the subspecies is unknown.

Note that $S$. $m$. hemprichii now refers to $S$. torquatus variegatus in Ash \& Atkins (2009). Moreover, S. t. armenicus in Ash \& Atkins (2009) is now treated as $S . m$. variegatus (Clements et al. 2015). Both subspecies are Palaearctic visitors in Ethiopia and Oromia (with few certain records of $S$. $m$. variegatus).

AH Note that S. t. variegatus in Ash \& Atkins (2009) now refers to S. maurus hemprichii. Moreover, S. t. armenicus in Ash \& Atkins (2009) is now treated as $S . m$. variegatus (Clements et al. 2015).

SM Geographically isolated subspecies with tiny range restricted to SW Ethiopia. Records only from Oromia and Southern Nations, Nationalities, and People's Regional State. A largely Sudan-Guinean species, but M. a. pachyrhycha more connected to the Somalia-Masai biome (cf. similar situation with Cossypha albicapillus omoensis).

Two subspecies in Ethiopia and Oromia. T. c. albiscapulata in $\mathrm{N}, \mathrm{W}$, $\mathrm{C}$ and NE (extension into Harar area), and T. c. subrufipennis is said to be in SW. Division line between them unknown (Ash \& Atkins 2009).

C. f. omoensis could also reach north westernmost Kenya (see maps in Lewis and Pomeroy 1989, Ash \& Atkins 2009).

SM C. scotocerca patchily distributed in C and NE Africa with isolated populations. C. s. turkana in S Oromia. Also isolated records from four 1-degree-tetrads in E Oromia, E of Bale, but affiliation of these birds

SM uncertain. Could be either C. s. turkana or C. s. spectatrix

(Ash \& Atkins 2009).

E, H SM C. dubia restricted to C and NE Ethiopia, with most records around Mt. Fantalle (triangle Oromia, Afar, Amhara). Only one old record from Somalia (Ash \& Atkins 2009). Single birds found in Djibouti and Somaliland in 2010 (Borrow 2010) and 2012 (Redman 2012)

E, H AH C. sordida patchily distributed in NE and E Africa with isolated populations. Nominate nearly restricted to Ethiopia. One recent first record from Eritrea in 1998 (Ash \& Atkins 2009).

H SM Restricted to Ethiopia and Somalia. In Ethiopia nearly all records from Somali National Regional State. Only one occupied 1-minute-tetrad in NE Oromia, more appear possible. Known altitudes in Ethiopia are $220-410 \mathrm{~m}$ (Ash \& Atkins 2009), but 600-1,200 m in N Somalia (del Hoyo et al. 2005).

Both subspecies are Palaearctic winter visitors and passage migrants, but their relative abundance and distribution is not understood (Ash \& Atkins 2009)

AH Formerly treated as subspecies of $O$. lugens. Restricted to Ethiopia and Eritrea, within Oromia in $\mathrm{C}$ and $\mathrm{N}$.

AH Nearly restricted to Ethiopia, with only few records from Eritrea (three 1-degree-tetrads, Ash \& Atkins 2009). Within Ethiopia nearly restricted to Amhara and Oromia.

Formerly regarded as subspecies of $O$. bottae. Map in

Ash \& Atkins (2009) with few records from NW Ethiopia and one in Eritrea. Only one occupied 1-minute-tetrad in SW Oromia (Lake Chew Bahir). Will be found more frequently in the future, because an unknown number of birds recorded in the past as $O$. bottae my actually have been O. heuglini.

AH Placed in genera Zoothera in Ash \& Atkins (2009). 


\begin{tabular}{|c|c|c|c|c|c|}
\hline $27343 / 675$ & $\begin{array}{l}\text { Psophocichla litsitsirupa simensis } \\
\text { (Rüppell, 1840) }\end{array}$ & $\mathrm{x}$ & $\mathrm{H}$ & $\mathrm{AH}$ & $\begin{array}{l}\text { P. l. simensis restricted to Ethiopia (widespread) and Eritrea (five oc- } \\
\text { cupied 1-degree-tetrads). Geographically isolated from the other three } \\
\text { subspecies, living from E Congo/Tanzania to S Africa. Genetic relation- } \\
\text { ships should be investigated. }\end{array}$ \\
\hline $27362 / 678$ & $\begin{array}{l}\text { Turdus abyssinicus abyssinicus } \\
\text { J. F. Gmelin, } 1789\end{array}$ & $\mathrm{x}$ & $\mathrm{H}$ & $\mathrm{AH}$ & $\begin{array}{l}\text { Referred to as Turdus olivaceus abyssinicus in Ash \& Atkins (2009). } \\
\text { Restricted to Ethiopia (widespread) and Eritrea (six occupied 1-degree- } \\
\text { tetrads). Range is very similar to that of } \\
\text { Psophocichla litsitsirupa simensis. }\end{array}$ \\
\hline $27386 / 676$ & $\begin{array}{l}\text { Turdus pelios pelios } \\
\text { Bonaparte, } 1850\end{array}$ & $\mathrm{x}$ & & SG & $\begin{array}{l}\text { Two subspecies of } T \text {. pelios in Ethiopia and Oromia. } T \text {. p. pelios widely } \\
\text { distributed, and } T \text {. } p \text {. centralis stated in SW, but } T \text {. } p \text {. centralis (darker } \\
\text { and and more heavily streaked on throat) poorly differentiated from }\end{array}$ \\
\hline $27392 / 676$ & $\begin{array}{l}\text { Turdus pelios centralis } \\
\text { Reichenow, } 1905\end{array}$ & $\mathrm{x}$ & & & nominate form (Ash \& Atkins 2009). \\
\hline $27989 / 662$ & $\begin{array}{l}\text { Cinnyricinclus leucogaster leucogaster } \\
\text { (Boddaert, 1783) }\end{array}$ & $\mathrm{x}$ & & & $\begin{array}{l}\text { C. leucogaster with all three subspecies in Ethiopia. C. l. leucogaster in } \\
\mathrm{C} \text { and } \mathrm{S} \text {, where presumably a breeding resident. C. l. verreauxi also in } \mathrm{C} \\
\text { and } \mathrm{S} \text {, where apparently an intra-tropical migrant visitor. C. l. arabicus }\end{array}$ \\
\hline $27990 / 662$ & $\begin{array}{l}\text { Cinnyricinclus leucogaster verreauxi } \\
\text { (Bocage, 1870) }\end{array}$ & $\mathrm{x}$ & & & $\begin{array}{l}\text { probably outside Oromia north of at least } 10^{\circ} \mathrm{N} \text {. In Ash \& Atkins' (2009) } \\
\text { map subspecies not separated. }\end{array}$ \\
\hline $27991 / 662$ & $\begin{array}{l}\text { Cinnyricinclus leucogaster arabicus } \\
\text { C. H. B. Grant \& Mackworth-Praed, } 1942\end{array}$ & ? & & & \\
\hline $27993 / 665$ & $\begin{array}{l}\text { Onychognathus tenuirostris tenuirostris } \\
\text { (Rüppell, 1836) }\end{array}$ & $\mathrm{x}$ & E, $\mathrm{H}$ & $\mathrm{AH}$ & $\begin{array}{l}\text { O. t. tenuirostris nearly restricted to Ethiopia, with only two occupied } \\
\text { 1-degree-tetrads in Eritrea (Ash \& Atkins 2009). }\end{array}$ \\
\hline $28001 / 664$ & $\begin{array}{l}\text { Onychognathus morio rueppellii } \\
\text { (J. Verreaux, 1856) }\end{array}$ & $\mathrm{x}$ & $\mathrm{H}+$ & SM & \\
\hline $28012 / 668$ & $\begin{array}{l}\text { Onychognathus albirostris } \\
\text { (Rüppell, 1836) }\end{array}$ & $\mathrm{x}$ & $\mathrm{H}$ & $\mathrm{AH}$ & $\begin{array}{l}\text { Endemic to Eritrea and Ethiopia, mainly north of } 8^{\circ} \mathrm{N} \text { and locally com- } \\
\text { mon there, uncommon in Bale Mts. }\end{array}$ \\
\hline $28013 / 667$ & $\begin{array}{l}\text { Onychognathus salvadorii } \\
\text { (Sharpe, 1891) }\end{array}$ & $\mathrm{x}$ & & SM & $\begin{array}{l}\text { Small range restricted to NE Africa, in S up to C Kenya. Rather patchily } \\
\text { distributed, in E and S Ethiopia locally common (Ash \& Atkins 2009). }\end{array}$ \\
\hline 28014 / 666 & $\begin{array}{l}\text { Onychognathus blythii } \\
\text { (Hartlaub, 1859) }\end{array}$ & $?$ & $\mathrm{H}$ & & $\begin{array}{l}\text { O. blythii occurs in N and NE Ethiopia. Also records from Oromia (Rift } \\
\text { valley, Bale Mts.), but not confirmed (Ash \& Atkins 2009). Not included } \\
\text { in Oromia checklist. }\end{array}$ \\
\hline $28021 / 671$ & $\begin{array}{l}\text { Speculipastor bicolor } \\
\text { Reichenow, } 1879\end{array}$ & $\mathrm{x}$ & & SM & $\begin{array}{l}\text { Small breeding range restricted to NE Africa, from Somalia to S and } \\
\text { E Ethiopia and N Kenya. Recorded nonbreeding from E Kenya and S } \\
\text { Somalia, but movements need investigation. }\end{array}$ \\
\hline $28022 / 670$ & $\begin{array}{l}\text { Pholia sharpii } \\
\text { (F. J. Jackson, 1898) }\end{array}$ & $\mathrm{x}$ & & $\mathrm{AH}$ & $\begin{array}{l}\text { Patchily distributed forest species, in Ethiopia only } \mathrm{W} \text { and } \mathrm{C} \text {. Ethiopian } \\
\text { population appears geographically isolated, next population in C Kenya. }\end{array}$ \\
\hline $28035 / 659$ & $\begin{array}{l}\text { Lamprotornis shelleyi } \\
\text { (Sharpe, 1890) }\end{array}$ & $\mathrm{x}$ & & SM & $\begin{array}{l}\text { Small breeding range restricted to NE Africa, from NW Somalia to S } \\
\text { Ethiopia, probably also SE South Sudan and N Kenya. Nonbreeding in } \\
\text { S Somalia and E Kenya to the Tanzanian border. Movements not fully } \\
\text { understood. }\end{array}$ \\
\hline $28058 / 663$ & $\begin{array}{l}\text { Lamprotornis albicapillus albicapillus } \\
\text { (Blyth, 1855) }\end{array}$ & $\mathrm{x}$ & $\mathrm{H}^{+}$ & SM & $\begin{array}{l}\text { Formerly treated as Spreo albicapillus. Both subspecies in Ethiopia. } \\
\text { Within Oromia L. a. albicapillus in S, and L. a. horrensis in Awash area } \\
\text { and maybe in the south-easternmost fringe. Dividing line between sub- } \\
\text { species unclear (Ash \& Atkins 2009). }\end{array}$ \\
\hline $28059 / 663$ & $\begin{array}{l}\text { Lamprotornis albicapillus horrensis } \\
\text { (Keith, 1964) }\end{array}$ & $\mathrm{x}$ & & SM & \\
\hline $28069 / 653$ & $\begin{array}{l}\text { Lamprotornis chalybaeus cyaniventris } \\
\text { Blyth, } 1855\end{array}$ & $\mathrm{x}$ & & & \\
\hline 28374 / 732 & $\begin{array}{l}\text { Hedydipna collaris djamdjamensis } \\
\text { (Benson, 1942) }\end{array}$ & $\mathrm{x}$ & E, $\mathrm{H}$ & SM & $\begin{array}{l}\text { Three subspecies in Ethiopia, two within Oromia. H. c. djamdjamensis } \\
\text { in C, E and S, H. c. garguensis in south- westernmost fringe (Lake Chew } \\
\text { Bahir, Ash \& Atkins 2009). }\end{array}$ \\
\hline $28375 / 732$ & $\begin{array}{l}\text { Hedydipna collaris garguensis } \\
\text { (Mearns, 1915) }\end{array}$ & $\mathrm{x}$ & & & \\
\hline $28420 / 735$ & $\begin{array}{l}\text { Cyanomitra obscura ragazzii } \\
\text { (Salvadori, 1888) }\end{array}$ & $\mathrm{x}$ & & & \\
\hline 28444 / 736 & $\begin{array}{l}\text { Chalcomitra senegalensis cruentata } \\
\text { (Rüppell, 1845) }\end{array}$ & $\mathrm{x}$ & & & \\
\hline $28448 / 737$ & $\begin{array}{l}\text { Chalcomitra hunteri hunteri } \\
\text { (Shelley, 1889) }\end{array}$ & $?$ & & SM & $\begin{array}{l}\text { Both subspecies said to be in S Ethiopia (Clements et al. 2015). In } \\
\text { Ash \& Atkins (2009) no differentiation, occurrence of the two subspe- } \\
\text { cies in Ethiopia and Oromia needs clarification. }\end{array}$ \\
\hline $28449 / 737$ & Chalcomitra hunteri siccata & $?$ & & SM & \\
\hline
\end{tabular}




\begin{tabular}{|c|c|c|c|}
\hline $28494 / 738$ & $\begin{array}{l}\text { Nectarinia tacazze tacazze } \\
\text { (Stanley, 1814) }\end{array}$ & $\mathrm{x}$ & $\mathrm{H}$ \\
\hline $28501 / 739$ & $\begin{array}{l}\text { Nectarinia famosa cupreonitens } \\
\text { Shelley, } 1876\end{array}$ & $\mathrm{x}$ & \\
\hline $28556 / 741$ & $\begin{array}{l}\text { Cinnyris pulchellus melanogastrus } \\
\text { (G. A. Fischer \& Reichenow, 1884) }\end{array}$ & $\mathrm{x}$ & \\
\hline $28558 / 742$ & $\begin{array}{l}\text { Cinnyris mariquensis osiris } \\
\text { (Finsch, 1870) }\end{array}$ & $\mathrm{x}$ & \\
\hline $28567 / 743$ & $\begin{array}{l}\text { Cinnyris nectarinioides erlangeri } \\
\text { (Reichenow, 1905) }\end{array}$ & $\mathrm{x}$ & $\mathrm{E}, \mathrm{H}^{+}$ \\
\hline $28580 / 745$ & $\begin{array}{l}\text { Cinnyris habessinicus habessinicus } \\
\text { (Hemprich \& Ehrenberg, 1828) }\end{array}$ & $\mathrm{x}$ & \\
\hline $28582 / 745$ & $\begin{array}{l}\text { Cinnyris habessinicus turkanae } \\
\text { van Someren, } 1920\end{array}$ & $\mathrm{x}$ & \\
\hline $28581 / 745$ & $\begin{array}{l}\text { Cinnyris habessinicus alter } \\
\text { Neumann, } 1906\end{array}$ & ? & \\
\hline $28602 / 746$ & $\begin{array}{l}\text { Cinnyris venustus fazoqlensis } \\
\text { (Heuglin, 1873) }\end{array}$ & $\mathrm{x}$ & \\
\hline $28604 / 746$ & $\begin{array}{l}\text { Cinnyris venustus albiventris } \\
\text { (Strickland, 1852) }\end{array}$ & $\mathrm{x}$ & \\
\hline $28854 / 833$ & $\begin{array}{l}\text { Motacilla flava lutea } \\
\text { (S. G. Gmelin, 1774) }\end{array}$ & $\mathrm{x}$ & \\
\hline $28855 / 833$ & $\begin{array}{l}\text { Motacilla flava thunbergi } \\
\text { Billberg, } 1828\end{array}$ & $\mathrm{x}$ & \\
\hline $28857 / 833$ & $\begin{array}{l}\text { Motacilla flava flava } \\
\text { Linnaeus, } 1758\end{array}$ & $\mathrm{x}$ & \\
\hline $28858 / 833$ & $\begin{array}{l}\text { Motacilla flava beema } \\
\text { (Sykes, 1832) }\end{array}$ & $\mathrm{x}$ & \\
\hline $28863 / 833$ & $\begin{array}{l}\text { Motacilla flava feldegg } \\
\text { Michahelles, } 1830\end{array}$ & $\mathrm{x}$ & \\
\hline $28864 / 833$ & $\begin{array}{l}\text { Motacilla flava leucocephala } \\
\text { (Przevalski, 1887) }\end{array}$ & $\mathrm{x}$ & \\
\hline $28891 / 836$ & $\begin{array}{l}\text { Motacilla clara clara } \\
\text { Sharpe, } 1908\end{array}$ & $\mathrm{x}$ & E, $\mathrm{H}$ \\
\hline $28894 / 837$ & $\begin{array}{l}\text { Motacilla alba alba } \\
\text { Linnaeus, } 1758\end{array}$ & $\mathrm{x}$ & \\
\hline $28895 / 837$ & $\begin{array}{l}\text { Motacilla alba dukhunensis } \\
\text { Sykes, } 1832\end{array}$ & $\mathrm{x}$ & \\
\hline $28909 / 838$ & $\begin{array}{l}\text { Motacilla aguimp vidua } \\
\text { Sundevall, } 1850\end{array}$ & $\mathrm{x}$ & \\
\hline $28945 / 841$ & $\begin{array}{l}\text { Anthus cinnamomeus cinnamomeus } \\
\text { Rüppell, } 1840\end{array}$ & $\mathrm{x}$ & E, $\mathrm{H}$ \\
\hline $28946 / 841$ & $\begin{array}{l}\text { Anthus cinnamomeus annae } \\
\text { Meinertzhagen, } 1921\end{array}$ & ? & \\
\hline $28955 / 841$ & $\begin{array}{l}\text { Anthus latistriatus } \\
\text { Jackson, } 1899\end{array}$ & ? & \\
\hline $28970 / 843$ & $\begin{array}{l}\text { Anthus similis nivescens } \\
\text { Reichenow, } 1905\end{array}$ & $\mathrm{x}$ & \\
\hline $28971 / 843$ & $\begin{array}{l}\text { Anthus similis hararensis } \\
\text { Neumann, } 1906\end{array}$ & $\mathrm{x}$ & E, $\mathrm{H}$ \\
\hline
\end{tabular}

AH N.t.tacazze restricted to Ethiopia and Eritrea. Geographically well separated from N. t. jacksoni in South Sudan, Kenya, and further S.

SM In Ethiopia only in SE Oromia (three 1-minute-tetrads in Ash \& Atkins 2009), and in S Somali National Regional State.

Separating line between C. h. habessinicus and C. h. turkanae in S Oromia, but their relative distributions unclear. Larger $C$. h. alter could reach Oromia in NE (Ash \& Atkins 2009).

$\mathrm{SM}$

SM

Within Ethiopia and Oromia C. v. fazoqlensis (yellow-bellied) in W and C. v. albiventris (white-bellied) in E. Distribution boundaries between them unclear, moreover, there is a wide wedge of overlap of the two SM subspecies in the southern Rift Valley (Ash \& Atkins 2009).

Six subspecies in Ethiopia and Oromia, all Palaearctic overwintering visitors and passage migrants. Proposed M. f. melanogrisea (mentioned by Ash \& Atkins 2009) not acknowledged by Clements et al. (2015).
E, H AH M. c. clara restricted to Ethiopia, except one record in Eritrea

(Ash \& Atkins 2009). Widespread in Oromia, but lacking in dry E and SE. Geographically isolated from other subspecies. Next populations as far as SW Kenya.

M. a. alba widespread Palaearctic overwintering visitor and passage migrant. M. a. dukhunensis said to be in $\mathrm{S}$, but only one certain record at Lake Langano (Ash \& Atkins 2009). A. c. cinnamomeus probably restricted to Ethiopia. Another subspecies,
A.c. annae, seems to be involved, but no clear evidence for Ethiopia or
Oromia. Could reach Oromia in north-easternmost fringes.

SM
Dark Anthus cinnamomeus known from Asosa (Benishangul-Gumuz Regional State) resemble A. latistriatus (Ash \& Akins 2009). Could also be expected in E Oromia. Further review is necessary. Not included in Oromia checklist.

Two subspecies considered to be in Ethiopia. Within Oromia A. s nivescens in the far NE, near the Somali border (three occupied 1-degreetetrads in Oromia) and A. s. hararensis elsewhere, except W. Seems nearly restricted to Ethiopia. Confident identification complicated, further review necessary. 


$\begin{array}{cl}28994 / 844 & \text { Anthus leucophrys omoensis } \\ & \text { Neumann, } 1906 \\ 28999 / 844 & \text { Anthus vaalensis saphiroi } \\ & \text { Neumann, } 1906 \\ 29094 / 847 & \text { Anthus caffer australoabyssinicus } \\ & \text { Benson, } 1942\end{array}$

$31493 / 864$

31494 / 865

$31500 / 867$

$31501 / 867$

$31556 / 871$

$32678 / 862$

Emberiza cineracea semenowi

Zarudny, 1904

Emberiza hortulana

Linnaeus, 1758

Emberiza striolata striolata

(Lichtenstein, 1823)

Emberiza striolata saturatior

(Sharpe, 1901)

Emberiza affinis omoensis

Neumann, 1905

Carduelis ankoberensis

(Ash, 1979)

$32721 / 857$

$32722 / 857$

$32727 / 850$

Serinus mozambicus grotei

W. L. Sclater \& Mackworth-Praed, 1931

Serinus mozambicus gommaensis

Grant \& Mackworth-Praed, 1945

Serinus citrinelloides citrinelloides

Rüppell, 1840

$32731 / 851$

Serinus hypostictus brittoni

Traylor, 1970

$32751 / 854$

Serinus xanthopygius

Rüppell, 1840

$32754 / 859$

Serinus dorsostriatus maculicollis

Sharpe, 1895

$32757 / 855$

Serinus flavigula

Salvadori, 1888

$32758 / 856$

Serinus xantholaemus

Salvadori, 1896

$32803 / 860$

Serinus reichardi striatipectus

(Sharpe, 1891)

Passer domesticus indicus

Jardine \& Selby 1831

$\mathrm{H}+$

Only A. l. omoensis in Ethiopia and Oromia. Widespread. Former subspecies A. leucophrys saphiroi (Ash \& Atkins 2009) now assigned to Anthus vaalensis (Clements et al. 2015).

SM Former A. leucophrys saphiroi (Ash \& Atkins 2009) now Anthus vaalensis saphiroi (Clements et al. 2015). Far NE Oromia, five occupied 1-degree-tetrads (Ash \& Atkins 2009).

O, E, H SM A. c. australoabyssinicus apparently endemic to Ethiopia and Oromia. Seems geographically isolated from other subspecies, next populations of A. c. blaymeyi in S Kenya. Few occupied 1-degree-tetrads with only nine records from S Oromia (Yabello-Mega area, Borana zone). One uncertain record in Somali National Regional State (Ash \& Atkins 2009). No further information.

Ethiopia incl. Oromia is one of the main wintering areas of this $\mathrm{W} \mathrm{Pa}-$ laearctic species. The majority of all individuals probably concentated here during the winter season.

Two disjunct subspecies in Ethiopia. Within Oromia E. s. striolata in NE, and E. s. saturatior in SW (Ash \& Atkins 2009).

E, $\mathrm{H}$

Nearly restricted to Ethiopia, with records from the border areas to Kenya and Sudan. Largely a Sudan-Guinean species, but E. a. omoensis not restricted to this biome.

E, $\mathrm{H} \quad \mathrm{AH}$ Endemic to Ethiopia (Amhara and most likely Oromia). Patchily distributed, only four occupied 1-degree-tetrads (Ash \& Atkins 2009), but maybe overlooked elsewhere. Favourite sites are Ankober and Simien maybe overlooked elsewhere. Favourite sites are Ankober and Simien al State). But Lemi as well as Ankober are close to the Amhara-Oromia border, the Lemi tetrad even covering mainly Oromia. Occurrence in Oromia therefore most likely but further investigation necessary.

Two subspecies in Ethiopia. Within Oromia $S$. m. gommaensis in NW (nearly restricted to Ethiopia), and S. $m$. grotei in SW. Line of division between the two is unclear (Ash \& Atkins 2009).

E, $\mathrm{H}+$

E, H AH Del Hoyo et al. (2010) recognize three more subspecies of

S. citronelloides, these separated by Clements et al. (2015) in two other species. S. c. citrinelloides nearly endemic to Ethiopia, with only three occupied 1-degree-tetrads in Eritrea (Ash \& Atkins 2009). Dowsett \& Dowsett-Lemaire (2105) point out that immature $S$. citrinelloides males might have been misidentified as $C$. hypostictus.

AM All birds in Ethiopia (incl. Oromia) most likely $S$. h. brittoni. No subspecific assignment by Ash \& Atkins (2009). No record since 1974. May only occur as a rare occasional vagrant, clarification of the status needed. Dowsett \& Dowsett-Lemaire (2105) doubt the occurrence of the species and point out that immature males of $S$. citrinelloides might have been misidentified as C. hypostictus.

AH Relatively poorly known species. Fairly common, but much overlooked or confused with C. reichenowi (Ash \& Atkins 2009). Main range in N Ethiopia and Eritrea. Only five occupied 1-degree-tetrads in central Oromia. Potential conspecificity with $S$. flavigula discussed by Mills \& Cohen (2015).

SM

E, H SM Tiny known range in central Ethiopia. Further research is almost certain to reveal its presence in other areas (Ash \& Atkins 2009). Formerly treated conspecific with $S$. xantholaemus. Birds on Mt. Fantalle with intreated conspecific with $S$. xantholaemus. Birds on Mt. Fantalle
termediate characters between S. flavigula and S. xantholaemus (del Hoyo et al. 2010). Provisionally considered a separate species pending further research (del Hoyo et al. 2010) but potential conspecificity with S. xanthopygius discussed by Mills \& Cohen (2015).

Small range. Patchily distributed in E and SE Oromia and the SW Somali Regional Zone, formerly treated conspecific with S. flavigula. Birds on Mt. Fantalle with intermediate characters between S. flavigula and S. xantholaemus (del Hoyo et al. 2010). Provisionally considered a separate species pending further research (del Hoyo et al. 2010).

According to Ash \& Atkins (2009) S. r. striatipectus may be a species in its own right, but no further explanation (del Hoyo et al. 2010). Only 18 records scattered over Ethiopia and Oromia.

Introduced. At Megado, near the Kenyan border, hybridisation $P$. d. indicus $\times$ P. castanopterus reported (Gedeon et al. 2015). 


\begin{tabular}{|c|c|c|c|c|c|}
\hline $32875 / 754$ & $\begin{array}{l}\text { Passer castanopterus castanopterus } \\
\text { Blyth, } 1855\end{array}$ & $?$ & $\mathrm{H}$ & SM & $\begin{array}{l}\text { Two disjunct subspecies in Ethiopia. Within Oromia only P. c. fulgens } \\
\text { in far SW (two occupied 1-degree-tetrads). } P \text {. c. castanopterus could be } \\
\text { expected in E Oromia, but no record so far (Ash \& Atkins 2009). }\end{array}$ \\
\hline $32876 / 754$ & $\begin{array}{l}\text { Passer castanopterus fulgens } \\
\text { Friedmann, } 1931\end{array}$ & $\mathrm{x}$ & & SM & \\
\hline $32893 / 755$ & $\begin{array}{l}\text { Passer shelleyi } \\
\text { Sharpe, } 1891\end{array}$ & $\mathrm{x}$ & & SM & $\begin{array}{l}\text { Patchily distributed in NE Africa, in Ethiopia only in far NE and S. } \\
\text { Connection between this populations unclear, in S restricted to Oromia } \\
\text { (Ash \& Atkins 2009). Poorly known species. }\end{array}$ \\
\hline $32903 / 756$ & $\begin{array}{l}\text { Passer griseus ugandae } \\
\text { Reichenow, } 1904 \\
\text { Passer swainsonii } \\
\text { (Rüppell, 1838) }\end{array}$ & $\mathrm{x}$ & $\mathrm{H}^{+}$ & $\mathrm{AH}$ & $\begin{array}{l}\text { Of the three closely related grey-headed sparrows in Ethiopia } \\
\text { P. swainsoni is widely distributed (except in SE). Overlaps with } \\
\text { P. griseus in W and P. gongonensis in S. Degree of ecological separation } \\
\text { and interbreeding, if any, is unknown. Further study needed } \\
\text { (Ash \& Atkins 2009). }\end{array}$ \\
\hline $32904 / 756$ & $\begin{array}{l}\text { Passer gongonensis } \\
\text { (Oustalet, 1890) }\end{array}$ & $\mathrm{x}$ & & SM & \\
\hline $32980 / 763$ & $\begin{array}{l}\text { Bubalornis niger intermedius } \\
\text { (Cabanis, 1868) }\end{array}$ & $\mathrm{x}$ & & SM & \\
\hline $32985 / 765$ & $\begin{array}{l}\text { Sporopipes frontalis frontalis } \\
\text { (Daudin, 1800) }\end{array}$ & $\mathrm{x}$ & & SG & \\
\hline $32989 / 748$ & $\begin{array}{l}\text { Plocepasser mahali melanorhynchus } \\
\text { Rüppell, } 1845\end{array}$ & $\mathrm{x}$ & & SM & \\
\hline $32995 / 750$ & $\begin{array}{l}\text { Plocepasser donaldsoni } \\
\text { Sharpe, } 1895\end{array}$ & $\mathrm{x}$ & & SM & $\begin{array}{l}\text { Sometimes treated within a separate Genus Fullerellus. Very small range } \\
\text { along the Kenyan-Oromia border, isolated records from S Somalia. } \\
\text { Poorly known species. }\end{array}$ \\
\hline $32998 / 751$ & $\begin{array}{l}\text { Pseudonigrita arnaudi arnaudi } \\
\text { (Bonaparte, 1850) }\end{array}$ & $\mathrm{x}$ & & & $\begin{array}{l}\text { P. arnaudi patchily distributed in E Africa. Proposed subspecies } \\
\text { P. a. australoabyssinicus for a geographically isolated population in } \mathrm{S} \\
\text { Oromia is treated as a synonym of nominate (del Hoyo et al. 2010). }\end{array}$ \\
\hline $33027 / 782$ & $\begin{array}{l}\text { Anaplectes rubriceps leuconotus } \\
\text { (J. W. von Müller, 1851) }\end{array}$ & $\mathrm{x}$ & & & \\
\hline $33035 / 767$ & $\begin{array}{l}\text { Ploceus baglafecht baglafecht } \\
\text { (Daudin, 1802) }\end{array}$ & $\mathrm{x}$ & $\mathrm{H}^{+}$ & $\mathrm{AH}$ & $\begin{array}{l}\text { According to del Hoyo et al. (2010) P. b. emini in SE Sudan, SW Ethio- } \\
\text { pia and N Uganda and also in C and S Ethiopia (Awasa and Yabello, van } \\
\text { den Elzen \& König 1983). However, according to Ash \& Atkins (2009) }\end{array}$ \\
\hline $33037 / 767$ & $\begin{array}{l}\text { Ploceus baglafecht emini } \\
\text { (Hartlaub, 1882) }\end{array}$ & $\mathrm{x}$ & & & $\begin{array}{l}\text { this subspecies within Ethiopia only in central parts, surrounded by and } \\
\text { mixed with two other subspecies }(P . b \text {. baglafecht and } P \text {. b. reichenowi). } \\
\text { Situation is complicated and requires detailed study. Many of these }\end{array}$ \\
\hline $33038 / 767$ & $\begin{array}{l}\text { Ploceus baglafecht reichenowi } \\
\text { (G. A. Fischer, 1884) }\end{array}$ & $\mathrm{x}$ & & $\mathrm{AH}$ & $\begin{array}{l}\text { weavers breed durıng the moult process. Therefore, the description of } \\
\text { new forms of "breeding birds" refers not always to the final breeding } \\
\text { plumage (van den Elzen \& König 1983). }\end{array}$ \\
\hline $33055 / 770$ & $\begin{array}{l}\text { Ploceus nigricollis melanoxanthus } \\
\text { (Cabanis, 1878) }\end{array}$ & $\mathrm{x}$ & & & $\begin{array}{l}\text { Population in SW Ethiopia seems to be small and is geographically iso- } \\
\text { lated. Next in C Kenya and S Somalia. }\end{array}$ \\
\hline $33058 / 769$ & $\begin{array}{l}\text { Ploceus ocularis crocatus } \\
\text { (Hartlaub, 1881) }\end{array}$ & $\mathrm{x}$ & & & $\begin{array}{l}\text { Population in Ethiopia geographically isolated. Next in S Kenya and } \\
\text { Uganda. }\end{array}$ \\
\hline $33087 / 774$ & $\begin{array}{l}\text { Ploceus intermedius intermedius } \\
\text { Rüppell, } 1845\end{array}$ & $\mathrm{x}$ & & SM & \\
\hline $33099 / 775$ & $\begin{array}{l}\text { Ploceus vitellinus uluensis } \\
\text { (Neumann, 1900) }\end{array}$ & $\mathrm{x}$ & & SM & \\
\hline $33105 / 776$ & $\begin{array}{l}\text { Ploceus spekei } \\
\text { (Heuglin, 1861) }\end{array}$ & $\mathrm{x}$ & & & $\begin{array}{l}\text { Remarkably patchily distributed throughout } \mathrm{E} \text { and NE Africa. Popula- } \\
\text { tions in some cases apparently isolated from each other. }\end{array}$ \\
\hline $33116 / 777$ & $\begin{array}{l}\text { Ploceus cucullatus abyssinicus } \\
\text { (J. F. Gmelin, 1789) }\end{array}$ & $\mathrm{x}$ & & & $\begin{array}{l}\text { One, probably two subspecies in Ethiopia. Within Oromia } \\
\text { P. c. abyssinicus widespread. Birds in far SE (one occupied 1-degree- } \\
\text { tetrad) most likely P. c. nigriceps. P. c. paropterus }\end{array}$ \\
\hline $33119 / 777$ & $\begin{array}{l}\text { Ploceus cucullatus nigriceps } \\
\text { (E. L. Layard, 1867) }\end{array}$ & $?$ & & & (map in Ash \& Atkins 2009) synonymised with the latter. \\
\hline $33133 / 780$ & $\begin{array}{l}\text { Ploceus rubiginosus rubiginosus } \\
\text { Rüppell, } 1840\end{array}$ & $\mathrm{x}$ & & SM & \\
\hline $33190 / 785$ & $\begin{array}{l}\text { Quelea quelea aethiopica } \\
\text { (Sundevall, 1850) }\end{array}$ & $\mathrm{x}$ & & & \\
\hline $33206 / 789$ & $\begin{array}{l}\text { Euplectes franciscanus } \\
\text { (Isert, 1789) }\end{array}$ & $\mathrm{x}$ & & & $\begin{array}{l}\text { E. f. pusillus, described from Lake Chew Bahir, is not acknowledged by } \\
\text { Clements et al. (2015). It often has longer tail-coverts, less red on breast, } \\
\text { and its plumage tending towards orange-red (del Hoyo et al. 2010) }\end{array}$ \\
\hline
\end{tabular}




\begin{tabular}{|c|c|c|c|c|c|}
\hline $33211 / 787$ & $\begin{array}{l}\text { Euplectes gierowii ansorgei } \\
\text { (E. J. O. Hartert, 1899) }\end{array}$ & $\mathrm{x}$ & & & $\begin{array}{l}\text { E. gierowi remarkably patchily distributed from Cameroun and Angola } \\
\text { to Kenya and Ethiopia. Some populations extremely isolated from each } \\
\text { other. }\end{array}$ \\
\hline $33216 / 786$ & $\begin{array}{l}\text { Euplectes afer strictus } \\
\text { Hartlaub, } 1857\end{array}$ & $\mathrm{x}$ & $\mathrm{H}+$ & $\mathrm{AH}$ & $\begin{array}{l}\text { Two subspecies in Ethiopia. Within Oromia E. a. strictus in C, and } \\
\text { E. a taha in SW (Ash \& Atkins 2009). Species patchily distributed } \\
\text { throughout Sub-Saharan Africa. }\end{array}$ \\
\hline $33217 / 786$ & $\begin{array}{l}\text { Euplectes afer taha } \\
\text { A. Smith, } 1836\end{array}$ & $\mathrm{x}$ & & & \\
\hline $33222 / 790$ & $\begin{array}{l}\text { Euplectes capensis xanthomelas } \\
\text { Rüppell, } 1840\end{array}$ & $\mathrm{x}$ & $\mathrm{E}, \mathrm{H}$ & $\mathrm{AH}$ & $\begin{array}{l}\text { E. c. xanthomelas endemic to Ethiopia. Geographically isolated from } \\
\text { other subspecies. }\end{array}$ \\
\hline $33229 / 793$ & $\begin{array}{l}\text { Euplectes albonotatus eques } \\
\text { (Hartlaub, 1863) }\end{array}$ & $\mathrm{x}$ & & & Population in Ethiopia geographically isolated. \\
\hline $33234 / 792$ & $\begin{array}{l}\text { Euplectes macroura macrocerca } \\
\text { (M. H. C. Lichtenstein, 1823) }\end{array}$ & $\mathrm{x}$ & & $\mathrm{AH}$ & \\
\hline $33237 / 794$ & $\begin{array}{l}\text { Euplectes ardens laticauda } \\
\text { (M. H. C. Lichtenstein, 1823) }\end{array}$ & $\mathrm{x}$ & $\mathrm{H}+$ & $\mathrm{AH}$ & E. a. laticauda geographically isolated from other subspecies. \\
\hline $33242 / 791$ & $\begin{array}{l}\text { Euplectes axillaris traversii } \\
\text { (Salvadori, 1888) }\end{array}$ & $\mathrm{x}$ & E, $\mathrm{H}$ & $\mathrm{AH}$ & $\begin{array}{l}\text { Two subspecies in Ethiopia. Within Oromia E. a. phoeniceus in W, and } \\
\text { E. a. traversi mainly in C. The subspecies intergrade presumably over a } \\
\text { large area west of the Rift Valley. More work is required to establish the } \\
\text { validity of the two subspecies (Ash \& Atkins 2009). }\end{array}$ \\
\hline $33243 / 791$ & $\begin{array}{l}\text { Euplectes axillaris phoeniceus } \\
\text { (Heuglin, 1862) }\end{array}$ & $\mathrm{x}$ & & & \\
\hline $33259 / 766$ & $\begin{array}{l}\text { Amblyospiza albifrons melanota } \\
\text { (Heuglin, 1863) }\end{array}$ & $\mathrm{x}$ & & & \\
\hline $33298 / 809$ & $\begin{array}{l}\text { Coccopygia quartinia quartinia } \\
\text { (Bonaparte, 1850) }\end{array}$ & $\mathrm{x}$ & $\mathrm{H}$ & $\mathrm{AH}$ & C. q. quartinia restricted to Ethiopia and Eritrea. \\
\hline $33306 / 799$ & $\begin{array}{l}\text { Mandingoa nitidula chubbi } \\
\text { (Ogilvie-Grant, 1912) }\end{array}$ & $\mathrm{x}$ & & & \\
\hline $33312 / 800$ & $\begin{array}{l}\text { Cryptospiza salvadorii salvadorii } \\
\text { Reichenow, } 1892\end{array}$ & $\mathrm{x}$ & & $\mathrm{AH}$ & $\begin{array}{l}\text { C. s. salvadori an apparently uncommon elusive breeding resident in } \\
\text { Ethiopia, most records from Oromia (Harenna forest, Wondo Genet, } \\
\text { above Shashemene, Ash \& Atkins 2009). C. s crystallochresta (de- } \\
\text { scribed from Challa, SW Ethiopia) synonymised with nominate form. }\end{array}$ \\
\hline $33325 / 810$ & $\begin{array}{l}\text { Estrilda paludicola ochrogaster } \\
\text { Salvadori, } 1897\end{array}$ & $\mathrm{x}$ & & & $\begin{array}{l}\text { E. p. ochrogaster sometimes considered a separate species } \\
\text { (del Hoyo et al. 2010). Restricted to Ethiopia and E Sudan. }\end{array}$ \\
\hline $33347 / 813$ & $\begin{array}{l}\text { Estrilda astrild peasei } \\
\text { Shelley, } 1903\end{array}$ & $\mathrm{x}$ & $\mathrm{E}, \mathrm{H}$ & & $\begin{array}{l}\text { Seems to be restricted to Ethiopia, only two recent unsubstantiated re- } \\
\text { cords from Eritrea. Also records near the border to Sudan and South } \\
\text { Sudan (Ash \& Atkins 2009). }\end{array}$ \\
\hline $33409 / 795$ & $\begin{array}{l}\text { Pytilia lineata } \\
\text { Heuglin, } 1863\end{array}$ & $\mathrm{x}$ & $\mathrm{E}, \mathrm{H}$ & SG & $\begin{array}{l}\text { The only Sudan-Guinea Savanna biome-restricted bird species endemic } \\
\text { to Ethiopia. According to Ash \& Atkins (2009) all records from Ethio- } \\
\text { pia, except one for Sudan near the Sudan-Ethiopian border. Formerly } \\
\text { occurred in E Sudan, where several records from close to the Ethiopian } \\
\text { border, but none since } 1910 \text { (del Hoyo et al. 2010). }\end{array}$ \\
\hline $33413 / 797$ & $\begin{array}{l}\text { Pytilia melba soudanensis } \\
\text { (Sharpe, 1890) }\end{array}$ & $\mathrm{x}$ & & & \\
\hline $33424 / 802$ & $\begin{array}{l}\text { Lagonosticta senegala rhodopsis } \\
\text { (Heuglin, 1863) }\end{array}$ & $\mathrm{x}$ & & & $\begin{array}{l}\text { Three subspecies of } L \text {. senegala in Ethiopia and Oromia. Delineation } \\
\text { between them on Ash \& Atkins' (2009) map only very approximate. Ap- } \\
\text { pear to occur close together. No details about ecologically separate or } \\
\text { overlapping areas. Studies needed. }\end{array}$ \\
\hline $33426 / 802$ & $\begin{array}{l}\text { Lagonosticta senegala brunneiceps } \\
\text { Sharpe, } 1890\end{array}$ & $\mathrm{x}$ & & & \\
\hline $33427 / 802$ & $\begin{array}{l}\text { Lagonosticta senegala somaliensis } \\
\text { Salvadori, } 1894\end{array}$ & $\mathrm{x}$ & & SM & \\
\hline $33430 / 801$ & $\begin{array}{l}\text { Lagonosticta rufopicta rufopicta } \\
\text { (Fraser, 1843) }\end{array}$ & $?$ & & SG & $\begin{array}{l}\text { According to Ash \& Atkins (2009) L. r. rufopicta in Ethiopia. However, } \\
\text { following Clements et al. (2015) this is } L \text {.r. lateritia. L. l. larvata geo- } \\
\text { graphically isolated from western subspecies. Main range of }\end{array}$ \\
\hline $33431 / 801$ & $\begin{array}{l}\text { Lagonosticta rufopicta lateritia } \\
\text { Heuglin, } 1864\end{array}$ & $?$ & & SG & L. l. larvata in Ethiopia, also in easternmost Sudan and South Sudan. \\
\hline $33436 / 805$ & Lagonosticta larvata larvata & $\mathrm{x}$ & $\mathrm{E}, \mathrm{H}+$ & SG & \\
\hline
\end{tabular}


$33442 / 803$

Lagonosticta rubricata congica

Sharpe, 1890

$33443 / 803$

Lagonosticta rubricata haematocephald

Neumann, 1907

$33456 / 798$

Amadina fasciata alexanderi

Neumann, 1908

$33479 / 816$

Ortygospiza fuscocrissa fuscocrissa

Heuglin, 1863

$33565 / 819$

Spermestes cucullata scutata

Heuglin, 1863

$33569 / 820$

Spermestes bicolor poensis

(Fraser, 1843)

$33577 / 817$

Euodice cantans orientalis

(Lorenz von Liburnau \& Hellmayr, 1901)

$33699 / 822$

Vidua chalybeata ultramarina

(J. F. Gmelin, 1789)

$33703 / 825$

Vidua wilsoni

(Hartert, 1901)

$33706 / 823$

Vidua raricola

Payne, 1982

$33707 / 824$

Vidua larvaticola

Payne, 1982

$33718 / 832$
E, $\mathrm{H} \quad \mathrm{AH} \quad$ Regarded as O. atricollis fuscocrissa in Ash \& Atkins (2009). On Ethiopian highlands, in Eritrea mainly along the Eritrean-Ethiopian border. Nearly endemic to Ethiopia (Ash \& Atkins 2009).
According to Ash \& Atkins (2009) L. r. ugandae (synonymized with L. r. congica), but following del Hoyo et al. (2010) and Clements et al. (2015) this is L. r. haematocephala.
Widely distributed in $\mathrm{C}, \mathrm{W}$ and $\mathrm{N}$ Ethiopia, but could be confused with other close related species ( $V$. wilsoni, V. raricola and V. larvaticola). More work is required (Ash \& Atkins 2009).

Only three records from 1970 and 1972 from one locality in W Ethiopia (Ash \& Atkins 2009). Next records from S South Sudan. Host is Lagonosticta rufopicta, a fairly common species in W Ethiopia.

Only one record in 1971, $38 \mathrm{~km}$ north of Yimma toward Suntu

(Ash \& Atkins 2009). Could be found elsewhere, because host of this parasitic species is Sporaeginthus subflavus, scattered widely in W Ethiopia. Next known population of $V$. raricola in southern South Sudan.

Only four records between 1926 and 1998 in W Ethiopia

(Ash \& Atkins 2009). According to the authors, two of these records could refer to V. camerunensis. Next records of V. larvaticola in E Sudan along the Sudan-Ethiopian border. Host is Lagonosticta larvata, a locally common species in W Ethiopia.

No subspecies assigned by Ash \& Atkins (2009), however, most likely A. i. imberbis. 